\title{
Activation of Chiral (Salen)AlCl Complex by Phosphorane for Highly Enantioselective Cyanosilylation of Ketones and Enones
}

\author{
Xing-Ping Zeng, ${ }^{1}$ Zhong-Yan Cao, ${ }^{1}$ Xin Wang, ${ }^{2}$ Long Chen, ${ }^{1}$ Feng Zhou, ${ }^{1}$ Feng Zhu, ${ }^{1}$ \\ Cui-Hong Wang, ${ }^{1}$ and Jian Zhou* ${ }^{1,3}$
}

${ }^{1}$ Shanghai Key Laboratory of Green Chemistry and Chemical Processes, School of Chemistry and Molecular

Engineering, East China Normal University, Shanghai, 200062, P R China; ${ }^{2}$ College of Chemistry, Sichuan University, Chengdu, 610064, P R China; ${ }^{3}$ State Key Laboratory of Organometallic Chemistry, Shanghai Institute of Organic Chemistry, Chinese Academy of Sciences, Shanghai 200032, P R China.

\begin{tabular}{|l|c|}
\hline Contents & Page \\
\hline 1. General information & $\mathrm{S} 2$ \\
\hline 2. Mechanistic studies & $\mathrm{S} 4$ \\
\hline 2-1. Control experiments and mechanistic consideration & $\mathrm{S} 4$ \\
\hline 2-2. NMR studies & \\
\hline 2-2-1. NMR analysis of the coordination of phosphorane 2a to complex 1 & $\mathrm{S} 7$ \\
\hline 2-2-2. Variable temperature ${ }^{31}$ P NMR experiments & $\mathrm{S} 11$ \\
\hline 2-3. Mass spectroscopy studies & $\mathrm{S} 31$ \\
\hline 2-4. In situ IR studies & $\mathrm{S} 34$ \\
\hline 2-5. DFT calculation results and discussion & $\mathrm{S} 39$ \\
\hline 2-6. Electrical conductivity experiments & $\mathrm{S} 46$ \\
\hline 3. Asymmetric cyanosilylation of ketone $\mathbf{8}$ & $\mathrm{S} 50$ \\
\hline 4. Tandem Wittig-cyanosilylation sequence using $\alpha, \beta$-unsaturated enals $\mathbf{1 0}$ & $\mathrm{S} 56$ \\
\hline 5. Tandem Wittig-cyanosilylation sequence using aliphatic aldehydes $\mathbf{3}$ & $\mathrm{S} 65$ \\
\hline 6. Elaboration and configuration determination of 7a & $\mathrm{S} 69$ \\
\hline 7. Single-crystal X-ray crystallography of $\mathbf{1 1} \& \mathbf{1 2}$ & $\mathrm{S} 73$ \\
\hline 8. Details for the DFT calculation & $\mathrm{S} 88$ \\
\hline 9. References & $\mathrm{S} 138$ \\
\hline
\end{tabular}




\section{General information}

Reactions were monitored by thin layer chromatography using UV light or $\mathrm{KMnO}_{4}$ to visualize the course of reaction. Purification of reaction products was carried out by flash chromatography on silica gel. Chemical yields refer to pure isolated substances. The $[\alpha]_{\mathrm{D}}$ was recorded using PolAAr 3005 High Accuracy Polarimeter. Infrared (IR) spectra were obtained using a Bruker tensor 27 infrared spectrometer. The in situ IR studies were conducted using ReactIR 45m and its Fiber Probe from METTLER TOLEDO. ${ }^{1} \mathrm{H},{ }^{13} \mathrm{C}$ and ${ }^{19} \mathrm{~F}$ NMR spectra were obtained using a Bruker DPX-400 spectrometer. Chemical shifts were reported in ppm from tetramethylsilane with the solvent resonance as the internal standard. The following abbreviations were used to designate chemical shift multiplicities: $\mathrm{s}=$ singlet, $\mathrm{d}=$ doublet, $\mathrm{t}=$ triplet, $\mathrm{q}=$ quartet, $\mathrm{h}=$ heptet, $\mathrm{m}=$ multiplet, $\mathrm{br}=$ broad .

All reactions were carried out under nitrogen sphere unless noted. Anhydrous $\mathrm{CH}_{2} \mathrm{Cl}_{2}$ was prepared by first pre-dried with anhydrous $\mathrm{Na}_{2} \mathrm{SO}_{4}$, then distilled from $\mathrm{P}_{2} \mathrm{O}_{5}$ into $\mathrm{CaH}_{2}$ and freshly from $\mathrm{CaH}_{2}$ distilled prior use. Anhydrous ethyl acetate was prepared by distillation over activated calcium sulfate and $5 \AA$ MS prior use. Anhydrous THF, toluene, $n$-hexane was prepared by distillation over sodium-benzophenone ketyl prior to use. Anhydrous acetone was prepared by first pre-dried with anhydrous $\mathrm{Na}_{2} \mathrm{SO}_{4}$, then distilled from $\mathrm{CaSO}_{4}$. (Salen)AlCl complexes $(S, S)$ - and $(R, R)$-1 were prepared according to a procedure reported by Jacobsen and co-workers. ${ }^{1}$ All the substrates were commercially available or prepared following literature reports. ${ }^{2}$

All DFT calculations were performed with Gaussian 09 program. ${ }^{3}$ The Becke's three-parameter nonlocal exchange functional ${ }^{4}$ and the Lee, Yang, and Parr nonlocal correlation functional ${ }^{5}$ (B3LYP) were used and the relativistic effective core potential $(\mathrm{ECP})^{6}$ for $\mathrm{Al}$ was employed. The standard 6-31G(d) basis set was employed for all the non-metal atoms and the basis function for Al was a standard LANL2DZ basis set. The geometries of reactants, intermediates (IM), transition states (TS), 
and products were fully optimized, followed by vibrational frequency calculations at the same levels of theory to obtain the zero-point energies (ZPE) and verify whether it was a minimum or a transition state. We also considered the bulk solvent effects on the reaction, some species were optimized in the dichloromethane with the polarized continuum model using the integral equation formalism variant (IEFPCM). ${ }^{7}$ The temperature-dependent enthalpy corrections and the entropy effects were computed at $298 \mathrm{~K}$ and 1 atmosphere of pressure. To estimate the change of net charge on the reaction species, natural bond orbital (NBO) ${ }^{8}$ analysis was carried out at the same level of theory.

\section{List of abbreviation:}

\begin{tabular}{ccc}
\hline Entry & Chemical name & Abbreviation \\
\hline 1 & Petroleum ether & PE \\
2 & $N, N$-dimethylformamide & DMF \\
3 & Tetrahydrofuran & THF \\
4 & Trimethylsilyl cyanide & TMSCN \\
5 & $p$-Toluenesulfonic acid & $p$-TsOH \\
\hline
\end{tabular}




\section{Mechanistic studies}

\section{2-1. Control experiments and mechanistic consideration}

To probe the role of each component of the three-catalyst system consisting of chiral (salen)AlCl complex $(R, R)$-1, phosphorane $\mathbf{2 a}$ and $\mathrm{Ph}_{3} \mathrm{PO}$, a series of control experiments based on cyanosilylation of $\alpha, \beta, \gamma, \delta$-unsaturated enone $\mathbf{6 a}$ were conducted, as summarized in Table S1.

Table S1. Control experiments based on the cyanosilylation of enone $\mathbf{6 a}$

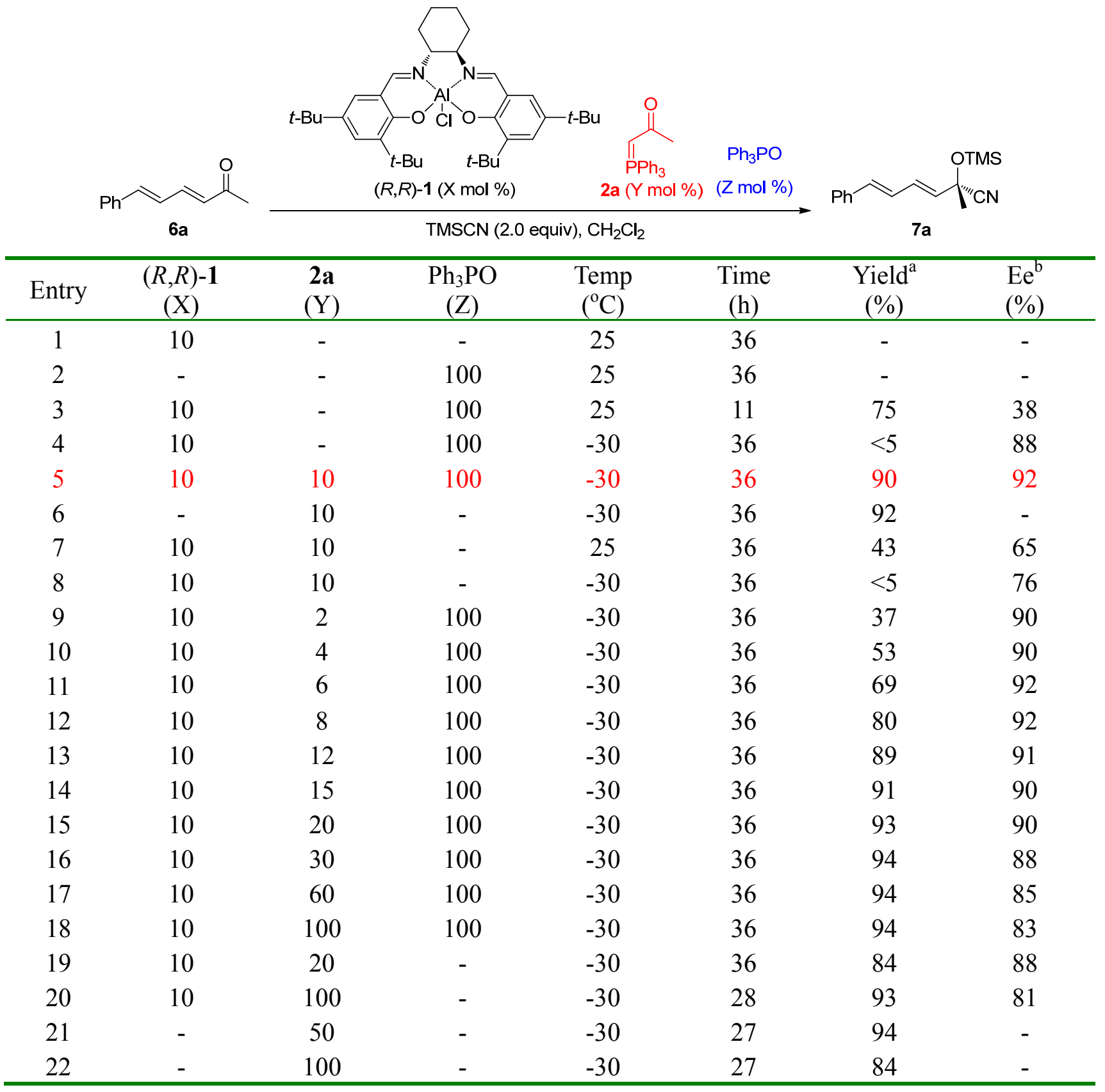

${ }^{\mathrm{a}}$ Isolated yield. ${ }^{\mathrm{b}}$ Determined by chiral HPLC analysis. 
First, as expected, no cyanosilylation took place either in the presence of $10 \mathrm{~mol} \%$ (salen)AlCl complex $(R, R)-1$ or $100 \mathrm{~mol} \% \mathrm{Ph}_{3} \mathrm{PO}$ (entries 1-2, Table S1). Although the merger of $10 \mathrm{~mol} \%$ $(R, R)-1$ and 100 mol \% $\mathrm{Ph}_{3} \mathrm{PO}$ could promote the reaction to finish within $11 \mathrm{~h}$ at $25{ }^{\circ} \mathrm{C}$, giving product $7 \mathbf{a}$ in $75 \%$ yield and $38 \%$ ee, it mediated the reaction very slowly at $-30{ }^{\circ} \mathrm{C}$ (entries $3-4$ ), demonstrating it impossible to achieve excellent enantioselectivity by lowering reaction temperature if using the dual catalyst system consisting of $(R, R)-\mathbf{1}$ and $\mathrm{Ph}_{3} \mathrm{PO}$. Considering there might be some remaining phosphorane 2a in the tandem Wittig-cyanosilylation sequence, we tried the addition of 10 mol \% 2a, and found the reaction was dramatically accelerated to afford product $7 \mathbf{a}$ in $90 \%$ yield, together with an improved ee of $92 \%$ (entry 5 ).

In the following control experiments to understand the role of phosphorane 2a, it was found that $10 \mathrm{~mol} \%$ of phosphorane $2 \mathrm{a}$ surprisingly mediated the reaction efficiently at $-30{ }^{\circ} \mathrm{C}$ to give $7 \mathbf{a}$ in $92 \%$ yield (entry 6). On the other hand, the combination of (salen)AlCl complex $\mathbf{1}$ and phosphorane $\mathbf{2 a}$ (10 mol \%, each) could catalyze the reaction at $25{ }^{\circ} \mathrm{C}$ to give $7 \mathrm{a}$ in only $43 \%$ yield and $65 \%$ ee after $36 \mathrm{~h}$, and poorly at $-30{ }^{\circ} \mathrm{C}$ (entries 7-8). These results indicated that dual system consisting of $(R, R)-\mathbf{1}$ and phosphorane $2 \mathbf{a}$ was impotent to achieve efficient cyanosilylation at $-30{ }^{\circ} \mathrm{C}$ as well (entry 8).

As phosphorane 2 a could mediate the cyanosilylation efficiently at $-30{ }^{\circ} \mathrm{C}$, experiments with different amount of $\mathbf{2 a}$ were further conducted to probe the influence of the ratio of $\mathbf{1}$ and $\mathbf{2 a}$ on the outcome. In the presence of $10 \mathrm{~mol} \%(R, R)-1$ and $100 \mathrm{~mol} \% \mathrm{Ph}_{3} \mathrm{PO}$, with the amount of phosphorane 2a raising from 2 to $10 \mathrm{~mol} \%$, equal to that of $(R, R)-\mathbf{1}$, the yield of $\mathbf{7 a}$ was obviously improved from $37 \%$ to $88 \%$, and the ee value was slightly increased from $90 \%$ to $92 \%$ (entries $9-12$ and 5). To our surprise, further increasing the amount of phosphorane $\mathbf{2 a}$ from $12 \mathrm{~mol} \%$ to 100 mol \%, the ee of product $7 \mathrm{a}$ only slightly decreased from $92 \%$ to $83 \%$ ee, and the reaction was not accelerated as fast as expected (entries 13-18). When excess phosphorane 2a existed, it was needless to use $\mathrm{Ph}_{3} \mathrm{PO}$ as co-catalyst, as the free $\mathbf{2 a}$ could activate TMSCN (entries 19-20). It also seemed that the presence of $\mathrm{Ph}_{3} \mathrm{PO}$ could to some extent suppress the achiral background reaction caused by phosphorane $\mathbf{2 a}$, as evidenced by the results obtained when $\mathbf{2 a}$ was two or ten times the amount of chiral complex $(R, R)-\mathbf{1}$ (entry 15 vs 19,18 vs 20 ).

On the other hand, when using $50 \mathrm{~mol} \%$ or $100 \mathrm{~mol} \%$ of phosphorane $2 \mathrm{a}$ to mediate the cyanosilylation of enone $\mathbf{6 a}$, no significant rate improvement was observed as compared with that mediated by $10 \mathrm{~mol} \%$ of $\mathbf{2 a}$ (entries $21-22$ vs entry 6 ). This observation suggested that phosphorane 2a might function as a trigger for the cyanosilylation, rather than a catalyst. ${ }^{9}$ The reason why the 
presence of much more amount of phosphorane 2a than that of $(R, R)-\mathbf{1}$ did not greatly decrease the enantioselectivity was unclear currently, possibly because the TMSCN activated by phosphorane 2a reacted with enone $\mathbf{6 a}$ bound to the aluminum center of chiral Lewis acid in a faster rate than with free enone. The detailed reason was now in investigation, as the mechanism how phosphorane $\mathbf{2 a}$ mediated the cyanosilylation waited for further studies.

These experiments also demonstrated it reproducible and convenient to develop the one-pot tandem Wittig-asymmetric cyanosilylation reaction, as excess phosphorane $\mathbf{2 a}$ did not greatly affect the enantioselectivity of the reaction, even if the Wittig reaction step did not consume all phosphorane 2a and there is substantial amount of phosphorane 2a remaining in the reaction system. Therefore, the Wittig reaction step of our tandem sequence was carried out using 1.1 equiv of phosphorane 2a and 1.0 equiv of aldehyde in a screw-capped pressure tube using $\mathrm{CH}_{2} \mathrm{Cl}_{2}$ as the solvent at $80{ }^{\circ} \mathrm{C}$, to ensure the almost full conversion of the Wittig step. By this procedure, at least 10 mol \% phosphorane 2a was present in the downstream asymmetric cyanosilylation, which was enough to maximize reactivity and enantioselectivity.

The above results cast some light on the reaction mechanism.

First, it suggested the coordination of phosphorane 2a to (salen) $\mathrm{AlCl}(R, R)-\mathbf{1}$ resulted in a more reactive Lewis acid species, as complex $(R, R)-\mathbf{1}$ itself could not catalyze cyanosilylation reaction at 25 ${ }^{\circ} \mathrm{C}$, but $(R, R)-\mathbf{1}$ in combination with phosphorane $2 \mathbf{a}$ could catalyze the reaction at $25{ }^{\circ} \mathrm{C}$ to give $7 \mathbf{a}$ in $43 \%$ yield and $65 \%$ ee, whereas the presence of free $2 \mathrm{a}$ would lead to racemic background reaction. However, it was not clear how phosphorane 2a coordinated to $(R, R)-\mathbf{1}$, as literature reports have shown that 2 a could form various $C$-coordinated (I) and $O$-coordinated (II) metal complexes. ${ }^{10}$ Although it might be anticipated that phosphorane 2a bound to Al via $O$-coordination due to hard-hard interaction, it required evidences for better understanding and how many molecules of $2 \mathbf{a}$ bound to complex $(R, R)-\mathbf{1}$ during the transition state should be investigated as well.
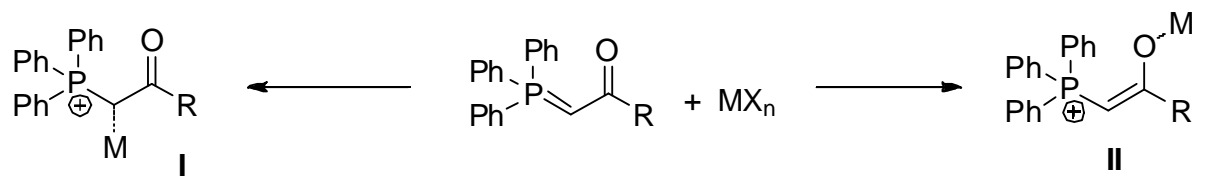

Second, it was possible that in the transition state, both a molecule of phosphorane 2a and enone 6a bound to aluminum, together with the tetradentate coordination of salen ligand to aluminum, as the well-known preferred coordination number of aluminum was six.

Third, the role of $\mathrm{Ph}_{3} \mathrm{PO}$ was possibly to act as a Lewis base to activate nucleophilic TMSCN, as 
there was no available coordinating site for $\mathrm{Ph}_{3} \mathrm{PO}$. It should be noted that the use of phosphine oxide as an additive in cyanosilylation reaction has a history date back to 1999, when Shibasaki et al. found that the addition of $\mathrm{Bu}_{3} \mathrm{PO}^{11 \mathrm{a}}$ enhanced the enantioselectivity of the asymmetric cyanosilylation of aliphatic aldehydes catalyzed by chiral $\mathrm{Al}$ complex. They proposed that the coordination of $\mathrm{Bu}_{3} \mathrm{PO}$ to the aluminum reduced its Lewis acidity and changed its geometry, which contributed to a more favorable transition state for enantiofacial control. Later in 2002, $\mathrm{Ph}_{3} \mathrm{PO}$ was reported to play a similar role ${ }^{11 \mathrm{~b}-\mathrm{d}}$. More recently in 2004, Corey et al. reported that $\mathrm{Ph}_{3} \mathrm{PO}$ could act as a Lewis base to cooperate with a chiral boron catalyst to achieve a highly enantioselective cyanohydrin formation reaction of aldehydes. ${ }^{12}$ Based on NMR experiments, they suggested that the role of $\mathrm{Ph}_{3} \mathrm{PO}$ was to activate TMSCN to form a more reactive isocyanide species $\mathrm{Ph}_{3} \mathrm{P}(\mathrm{OTMS})(\mathrm{N}=\mathrm{C}$ :). They also reported the IR analysis of a mixture of TMSCN and $\mathrm{Ph}_{3} \mathrm{PO}$, which showed a new -NC stretching band at 2072 $\mathrm{cm}^{-1}$, different from that of TMSCN $\left(2192 \mathrm{~cm}^{-1}\right)$ and TMSNC $\left(2095 \mathrm{~cm}^{-1}\right)$.

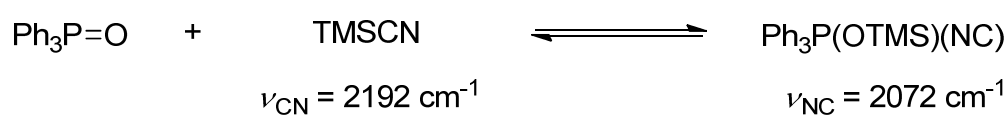

In the following, NMR studies, MS analysis, react IR studies, DFT calculations and electrical conductivity experiments were conducted to test these initial mechanistic insight.

\section{2-2. NMR studies}

The purpose of NMR experiments was to investigate the coordination of phosphorane $\mathbf{2 a}$ to aluminum of chiral (salen) $\mathrm{AlCl}$ complex $(R, R)-\mathbf{1}$, competitive coordination of phosphorane $\mathbf{2 a}, \mathrm{Ph}_{3} \mathrm{PO}$ and enone $6 \mathbf{a}$ to aluminum in the transition state.

\section{2-2-1. NMR analysis of the coordination of phosphorane 2a to complex $(R, R)-1$}

Navarro et al. reported the synthesis and characterization of a series of $\mathrm{Pd}(\mathrm{II})$ complexes using phosphorane $\mathbf{2 a}$ as the co-ligand, including both $C$-coordinated complexes and $O$-coordinated complexes. ${ }^{13}$ The characteristic peaks that distinguished $C$-coordinated complex from $O$-coordinated complex were highlighted in Table S2.

Useful information included the change of the chemical shift of $\mathrm{H}^{3}$ on ${ }^{1} \mathrm{H}$ NMR spectra and that of $\mathrm{C}^{2}$ and $\mathrm{C}^{3}$ on ${ }^{13} \mathrm{C}$ NMR spectra. The doublet peak of $\mathrm{H}^{3}$ at $\delta 3.69 \mathrm{ppm}$ would merge into a broad singlet peak at 4.51 if phosphorane 2a bound to $\mathrm{Pd}(\mathrm{II})$ in a $C$-coordinating fashion (entry 1 vs 2 , Table S2), while kept as a doublet peak at 3.98 if in a $O$-coordinating fashion (entry 1 vs 3 ). On the other 
hand, the doublet peak of $\mathrm{C}^{3}$ at $\delta 51.1 \mathrm{ppm}$ would greatly shifted to 28.5 if phosphorane $\mathbf{2 a}$ bound to $\operatorname{Pd}(\mathrm{II})$ in a $C$-coordinating fashion (entry 1 vs 2 ), while slightly shifted to 62.1 if in a $O$-coordinating fashion (entry 1 vs 3 ). Schweizer and Calcagno reported that the $O$-alkylated vinylphosphonium salt derived from $2 \mathrm{a}$ had a signal of $\mathrm{C}^{2}$ at 178.9 , along with the characteristic doublet peak of vinyl proton at $\delta 5.56 \mathrm{ppm}$, and a doublet peak of $\mathrm{C}^{3}$ at 76.5 (entry 4), which were more similar to those of the $O$-coordinated complex shown in entry $3 .^{14}$

Table S2. Reported data for phosphorane 2a derived metal complex

\begin{tabular}{|c|c|c|c|c|c|}
\hline Entry & Compound & $\begin{array}{c}{ }^{1} \mathrm{H} \operatorname{NMR}\left(\mathrm{H}^{3}\right) \\
(\mathrm{ppm})\end{array}$ & $\begin{array}{c}{ }^{13} \mathrm{C} \text { NMR }\left(\mathrm{C}^{2}\right) \\
(\mathrm{ppm})\end{array}$ & $\begin{array}{c}{ }^{13} \mathrm{C} \text { NMR }\left(\mathrm{C}^{3}\right) \\
(\mathrm{ppm})\end{array}$ & $\begin{array}{l}{ }^{31} \text { P NMR } \\
\text { (ppm) }\end{array}$ \\
\hline 1 & & 3.69 (d) & 190.54 & $51.12(\mathrm{~d})$ & 14.75 \\
\hline & & $4.51(\mathrm{~s}, \mathrm{br})$ & 204.28 & $28.53(d)$ & 25.62 \\
\hline $\begin{array}{c}3 \\
(O-\text {-coordination })\end{array}$ & & 3.98 (d) & 191.01 & $62.12(d)$ & $\begin{array}{l}14.19 / 14.07 \\
\text { (cis\&trans) }\end{array}$ \\
\hline 4 & $\mathrm{Br}^{\stackrel{\mathrm{Ph}_{3} \mathrm{P}}{ }}$ & $5.56(d)$ & 178.9 & 76.5 & -- \\
\hline 5 & $\begin{array}{c}(R, R)-\mathbf{1}+\mathbf{2 a} \\
(1: 1)\end{array}$ & $4.31(\mathrm{~d})$ & 184.00 & $67.28(d)$ & 12.76 \\
\hline
\end{tabular}

In our case, the ${ }^{1} \mathrm{H},{ }^{13} \mathrm{C}$ and ${ }^{31} \mathrm{P}$ NMR studies $\left(400 \mathrm{MHz}\right.$ for ${ }^{1} \mathrm{H}, 100 \mathrm{MHz}$ for ${ }^{13} \mathrm{C}$ and $202 \mathrm{MHz}$ for ${ }^{31} \mathrm{P}$ ) were recorded at room temperature in $\mathrm{CD}_{2} \mathrm{Cl}_{2}$ from Cambridge Isotope Laboratories, Tnc, which was used as received. The general procedure was as follows: in a $5 \mathrm{~mm}$ NMR tube containing $0.5 \mathrm{~mL} \mathrm{CD} \mathrm{Cl}_{2}$ was sequentially added to the corresponding compounds. After the solid was fully dissolved, the mixture was balanced at room temperature for half an hour before detection. The ${ }^{31} \mathrm{P}-\mathrm{NMR}$ study was conducted using $\mathrm{H}_{3} \mathrm{PO}_{4}(\delta=0.00 \mathrm{ppm})$ as the external standard.

As shown in Table $\mathrm{S} 3$, when mixing $\mathbf{2 a}$ with complex $(R, R)-\mathbf{1}$ in a 1:1 ratio, ${ }^{1} \mathrm{H}-\mathrm{NMR}$ analysis 
revealed that the signal of $\mathrm{H}^{3}$ of $\mathbf{2 a}$ shifted from $\delta 3.69 \mathrm{ppm}$ to 4.31 , but still was a double peak. The corresponding ${ }^{13} \mathrm{C}$ NMR analysis showed that the signal of $\mathrm{C}^{3}$ of $\mathbf{2 a}$ shifted from $51.11 \mathrm{ppm}$ to 67.28 , the signal of $\mathrm{C}^{3}$ of $2 \mathrm{a}$ shifted from 190.54 to 184.0 . ${ }^{31} \mathrm{P}$ NMR spectrum showed an up shift of the phosphine of 2a from $14.75 \mathrm{ppm}$ to 12.76 (major), with other two small peaks at 15.73 and 20.17 possibly due to the presence of isomers. All these trends supported the binding of $\mathbf{2 a}$ to $(R, R)-\mathbf{1}$ was in an $O$-coordination fashion, as described as follows.

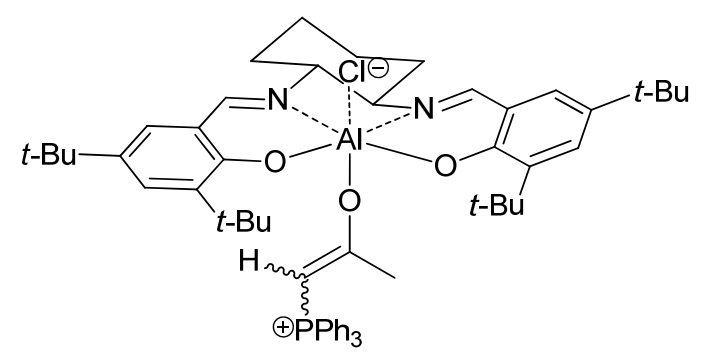


Table S3. NMR spectra of 2a, $(R, R)-\mathbf{1}$ and the 1:1 mixture of $\mathbf{2 a}$ and $\mathbf{1}$ (at $298 \mathrm{~K}$ )

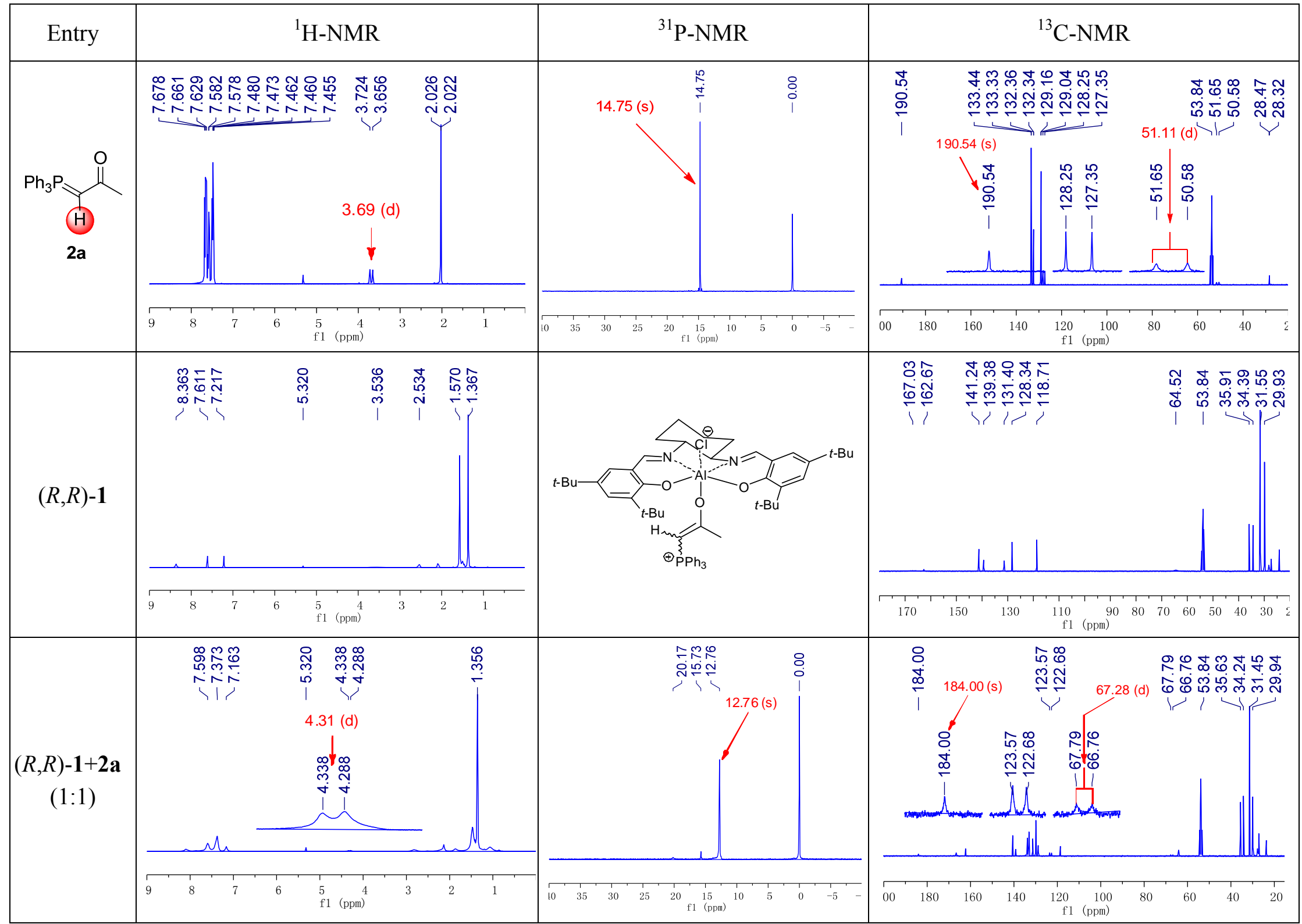




\section{2-2-2. Variable temperature ${ }^{31} \mathrm{P}$ NMR experiments}

Our reaction system included $10 \mathrm{~mol} \%(R, R)-\mathbf{1}, 10 \mathrm{~mol} \%$ phosphorane 2a, 1.0 equiv of $\mathrm{Ph}_{3} \mathrm{PO}$, 1.0 equiv of enone 6a and 2.0 equiv of TMSCN. Therefore, ${ }^{31} \mathrm{P}$ NMR analysis was straightforward to investigate the competitive coordination of phosphorane $2 \mathrm{a}$ and $\mathrm{Ph}_{3} \mathrm{PO}$ to complex $(R, R)-\mathbf{1}$. The ${ }^{31} \mathrm{P}$ NMR analysis was carried out by using $\mathrm{CD}_{2} \mathrm{Cl}_{2}$ as the solvent. However, when NMR experiments were conducted at $25{ }^{\circ} \mathrm{C}$, although obvious changes were observed, it was hard to distinguish the information corresponding to bounded and dissociated free ligand, due to the fast ligand-exchanging processes. Accordingly, variable temperature ${ }^{31} \mathrm{P}$ NMR $(202 \mathrm{MHz})$ spectra were recorded at $20{ }^{\circ} \mathrm{C}, 0$ ${ }^{\circ} \mathrm{C},-15{ }^{\circ} \mathrm{C},-30{ }^{\circ} \mathrm{C},-45{ }^{\circ} \mathrm{C}$ and $-55{ }^{\circ} \mathrm{C}$, respectively.

It should be noted that we did not use the titration experiments by adding compounds to the NMR tube in order to better mimic the reaction process. All NMR experiments discussed in this section were run as follows: 1) all the compounds for a given NMR experiment were dissolve in $\mathrm{CD}_{2} \mathrm{Cl}_{2}$, and the resulting mixture was stirred at room temperature for half an hour, as we wish to obtain the information of thermodynamic stable intermediates; 2) the resulting mixture was transferred to a NMR tube, and subjected for NMR analysis; 3) after the information at $20{ }^{\circ} \mathrm{C}$ was recorded, the temperature was gradually lowered down to the indicated temperature to get the corresponding information.

The following discussion were based on the spectra obtained at $218 \mathrm{~K}\left(-55^{\circ} \mathrm{C}\right)$, which was vivid enough to show the changes, but the full spectra were provided at the end of this section (Figures S5-S18).

First, the coordination of phosphorane 2a to $(R, R)-\mathbf{1}$ was analyzed (Figure S1). At $218 \mathrm{~K}$, the signal of free $\mathbf{2 a}$ was at $14.12 \mathrm{ppm}$ (i). The 1:1 mixture of the $\mathbf{2 a}$ and $(R, R)-\mathbf{1}$ showed a major peak at $12.40 \mathrm{ppm}$, and two peaks at 19.31 and $13.06 \mathrm{ppm}$, possibly due to the presence of isomers resulting from $O$-coordination (ii). The weak signal at $11.70 \mathrm{ppm}$ was possibly due to the binding of two molecules of phosphorane 2a to aluminum of complex $(R, R)-\mathbf{1}$, as it was the only signal of the 2:1 mixture of the 2a and $(R, R)-\mathbf{1}$ (iii). In addition, the 4:1 mixture of $\mathbf{2 a}$ and $(R, R)-\mathbf{1}$ had two distinct peaks, the signal at $14.05 \mathrm{ppm}$ corresponding to the free phosphorane $\mathbf{2 a}$, and the other at 11.50 corresponding to the bounded phosphorane $\mathbf{2 a}$ (iv). This analysis revealed that phosphorane $\mathbf{2 a}$ could readily coordinate to $(R, R)-\mathbf{1}$ to develop two complex, i.e. $\mathbf{2 a} /(R, R)-\mathbf{1}$ and $\mathbf{2 a} /(R, R)-\mathbf{1} / \mathbf{2} \mathbf{a}$. For the full spectra of variable temperature ${ }^{31} \mathrm{P}$ NMR analysis of $\mathbf{2 a}, 1: 1,2: 1$ and $4: 1$ mixture of the $\mathbf{2 a}$ and $(R, R)-\mathbf{1}$, see Figures S5-S8. 
Figure S1. ${ }^{31} \mathrm{P}$ NMR studies into the coordination of phosphorane $\mathbf{2 a}$ to $(R, R)-\mathbf{1}$ at $218 \mathrm{~K}$

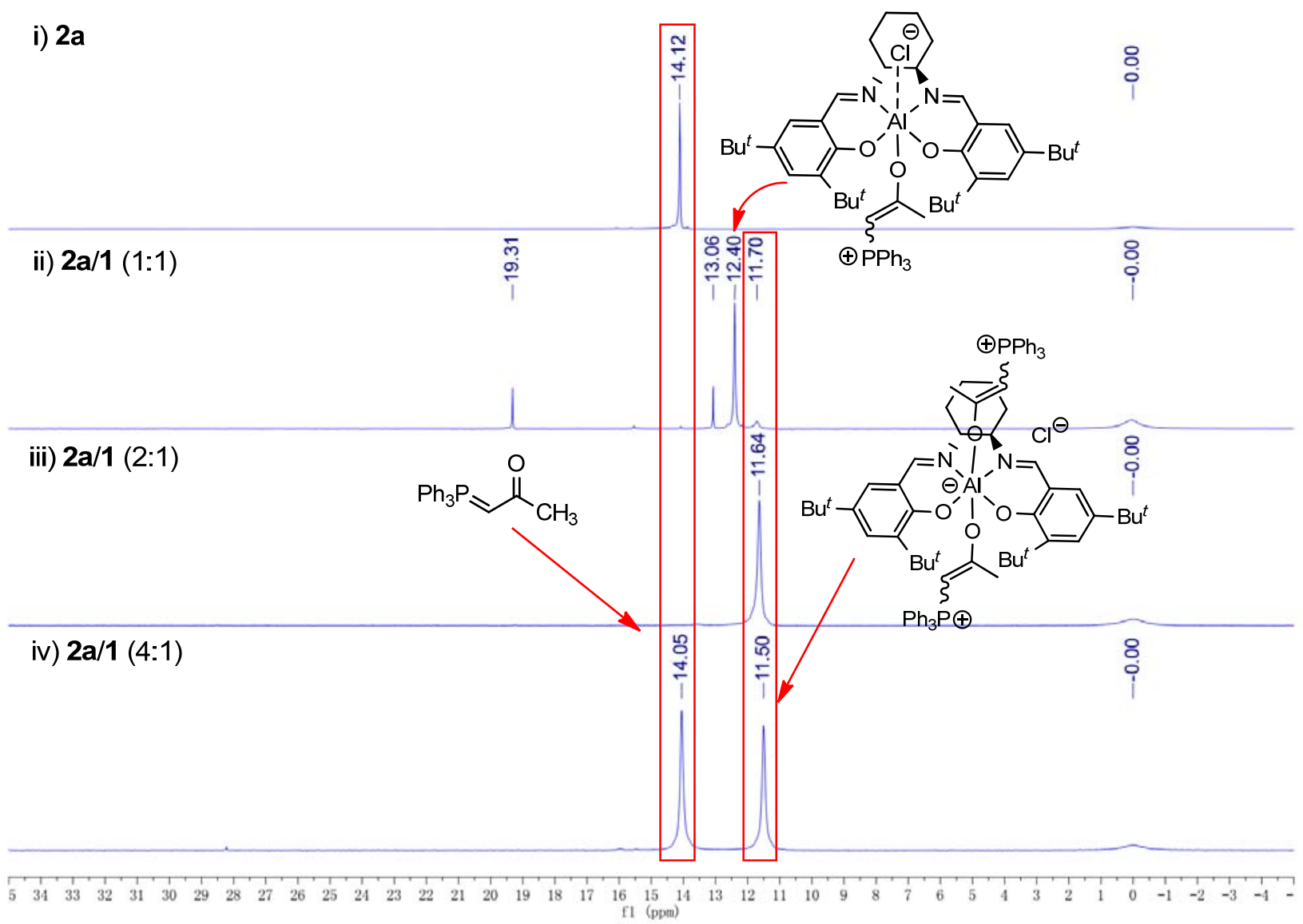

We then investigated the coordination of $\mathrm{Ph}_{3} \mathrm{PO}$ to $(R, R)-\mathbf{1}$ (Figure $\mathrm{S} 2$ ). At $218 \mathrm{~K}$, the signal of $\mathrm{Ph}_{3} \mathrm{PO}$ located at $28.56 \mathrm{ppm}$ (i). The 1:1 mixture of the $\mathrm{Ph}_{3} \mathrm{PO}$ and $(R, R)-1$ showed a major peak at $31.89 \mathrm{ppm}$ that might be the signal of $1: 1$ complex of $\mathrm{Ph}_{3} \mathrm{PO} /(R, R)-\mathbf{1}(\mathrm{ii})$. The weak signal at 33.32 ppm was not attributed at this stage. The spectra of the 2:1 mixture of $\mathrm{Ph}_{3} \mathrm{PO}$ and $(R, R)-1$ contained a similar major peak at $31.69 \mathrm{ppm}$ (iii), together with a signal at 28.53 corresponding to the free $\mathrm{Ph}_{3} \mathrm{PO}$, which suggested that there was still only one molecule of $\mathrm{Ph}_{3} \mathrm{PO}$ coordinating to $(R, R)-1$. The spectra of the 4:1 and 10:1 mixture of the $\mathrm{Ph}_{3} \mathrm{PO}$ and $(R, R)-1$ showed two distinct peaks (iv \& $\mathrm{v}$ ). The signal at around $31.8 \mathrm{ppm}$ should be attributed to the bounded $\mathrm{Ph}_{3} \mathrm{PO}$, and the other at around 28.5 was corresponding to the free $\mathrm{Ph}_{3} \mathrm{PO}$. Unexpectedly, the weak signal at around $33 \mathrm{ppm}$ disappeared in both cases, which was the reason why we believed that $\mathrm{Ph}_{3} \mathrm{PO}$ was more likely to coordinate to complex $(R, R)-\mathbf{1}$ to give a 1:1 complex of $\mathrm{Ph}_{3} \mathrm{PO} /(R, R)-\mathbf{1}$ regardless of their ratios, possibly due to steric hindrance. For full spectra of variable temperature ${ }^{31} \mathrm{P}$ NMR analysis of $\mathrm{Ph}_{3} \mathrm{PO}, 1: 1,2: 1,4: 1$ and 10:1 mixture of $\mathrm{Ph}_{3} \mathrm{PO}$ and $(R, R)$-1, see Figures $\mathrm{S} 9-\mathrm{S} 13$. 
Figure S2. ${ }^{31} \mathrm{P}$ NMR study of the coordination of $\mathrm{Ph}_{3} \mathrm{PO}$ to $(R, R)-1$ at $218 \mathrm{~K}$

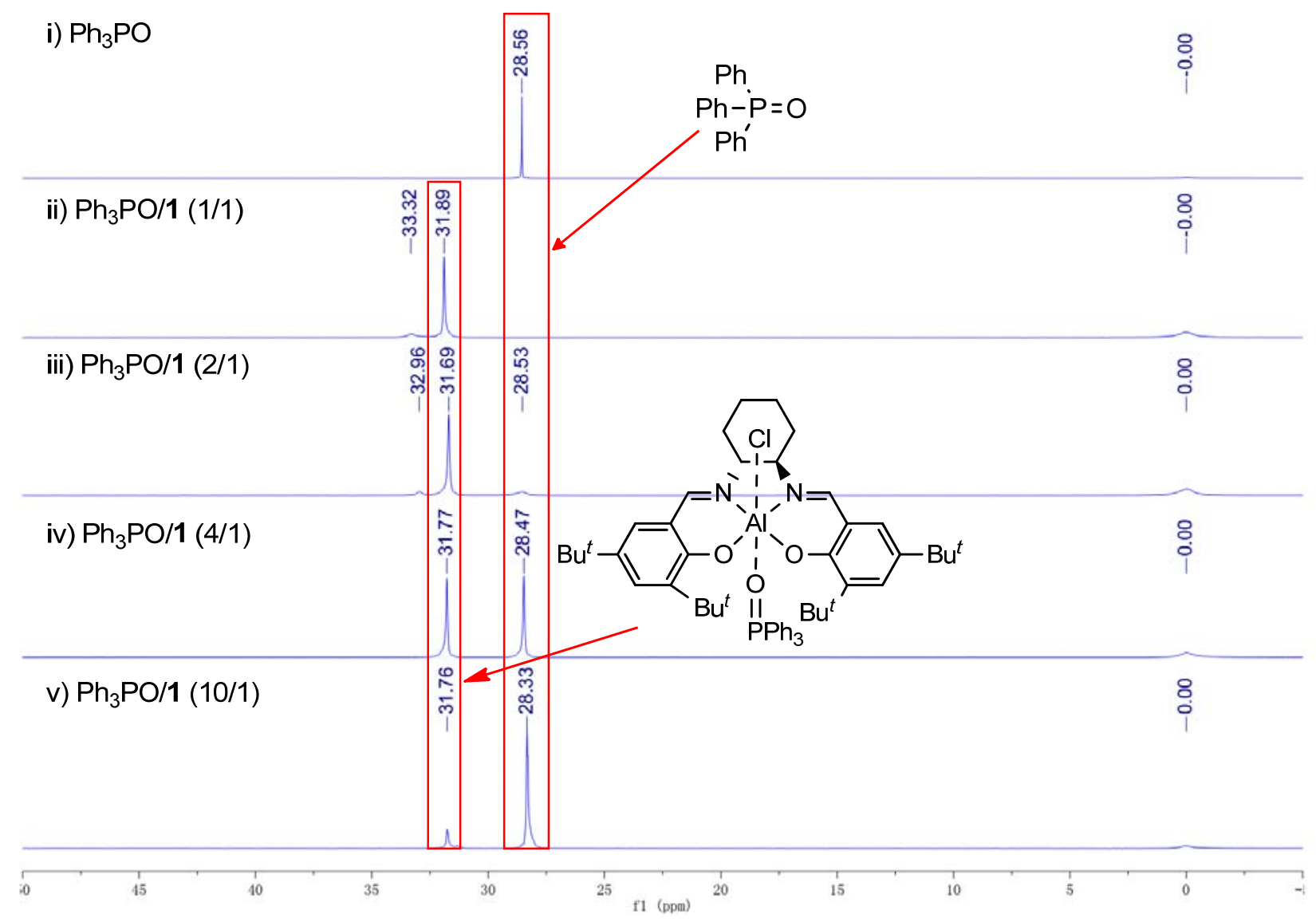

Since both phosphorane 2a and $\mathrm{Ph}_{3} \mathrm{PO}$ could coordinate to chiral complex $(R, R)-\mathbf{1}$, we then studied the possible competitive coordination of $\mathrm{Ph}_{3} \mathrm{PO}$ and $\mathbf{2 a}$ to $(R, R)-\mathbf{1}$, which were all present in the reaction system (Figure S3). First, the spectra of the 1:1:1 mixture of $\mathrm{Ph}_{3} \mathrm{PO}, \mathbf{2 a}$ and $(R, R)-\mathbf{1}(\mathrm{v})$ showed the signal of coordinated $\mathrm{Ph}_{3} \mathrm{PO}(\delta=31.74 \mathrm{ppm})$, free $\mathrm{Ph}_{3} \mathrm{PO}(\delta=28.81 \mathrm{ppm})$ and several peaks $(\delta=11.66-11.88 \mathrm{ppm})$, which was different from those observed in the spectrum of $\mathbf{2 a} /(R, R)-\mathbf{1}$ $(1: 1)$ complex (iii). As no signal correlating to free phosphorane $\mathbf{2 a}$ was observed, we attributed these peaks to the signal of the phosphine of the bounded phosphorane $\mathbf{2 a}$ in a newly formed $\mathbf{2 a} /(R, R)-\mathbf{1} / \mathrm{Ph}_{3} \mathrm{PO}(1: 1: 1)$ complex. Increasing the amount of $\mathrm{Ph}_{3} \mathrm{PO}$ to 10.0 equivalents caused a slightly up shift of the multiple peak to $11.56 \mathrm{ppm}$ (vi). It should be noted that in spectra $v$ and $v i$, no sign of free phosphorane was observed, supporting that phosphorane $\mathbf{2 a}$ coordinated to $(R, R)-\mathbf{1}$ even in the presence of 10.0 equiv of $\mathrm{Ph}_{3} \mathrm{PO}$. The variable temperature ${ }^{31} \mathrm{P}$ NMR spectra of $1: 1: 1$ and 10:1:1 mixture of $\mathrm{Ph}_{3} \mathrm{PO}, \mathbf{2 a}$ and $(R, R)-\mathbf{1}$ were presented in Figures S14-S15. 
Figure S3. ${ }^{31} \mathrm{P}$ NMR study of the competitive coordination of $\mathrm{Ph}_{3} \mathrm{PO}$ and $\mathbf{2 a}$ to $(R, R)-\mathbf{1}$ at $218 \mathrm{~K}$

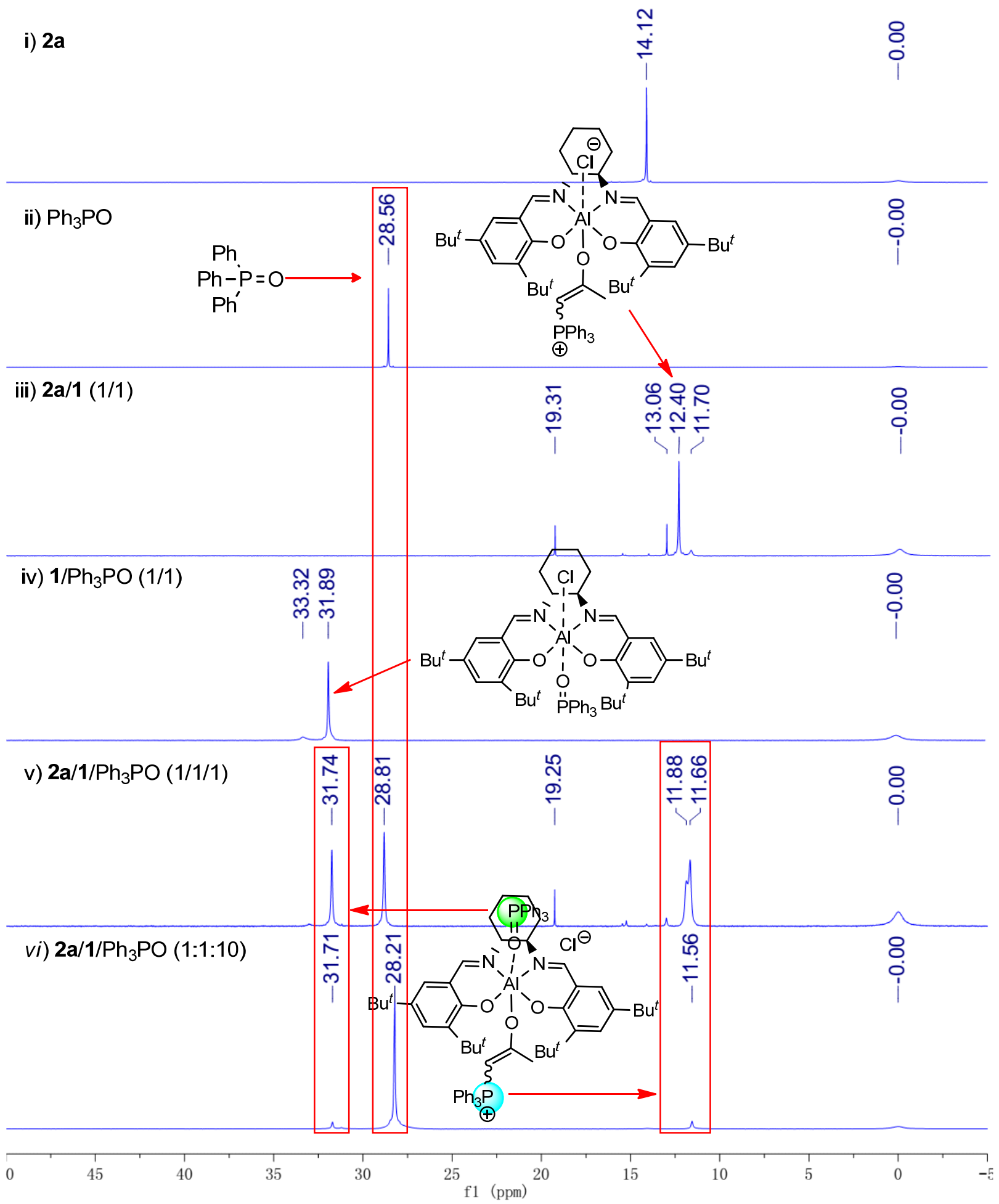


Figure S4. ${ }^{31} \mathrm{P}$ NMR study of reaction system at $218 \mathrm{~K}$

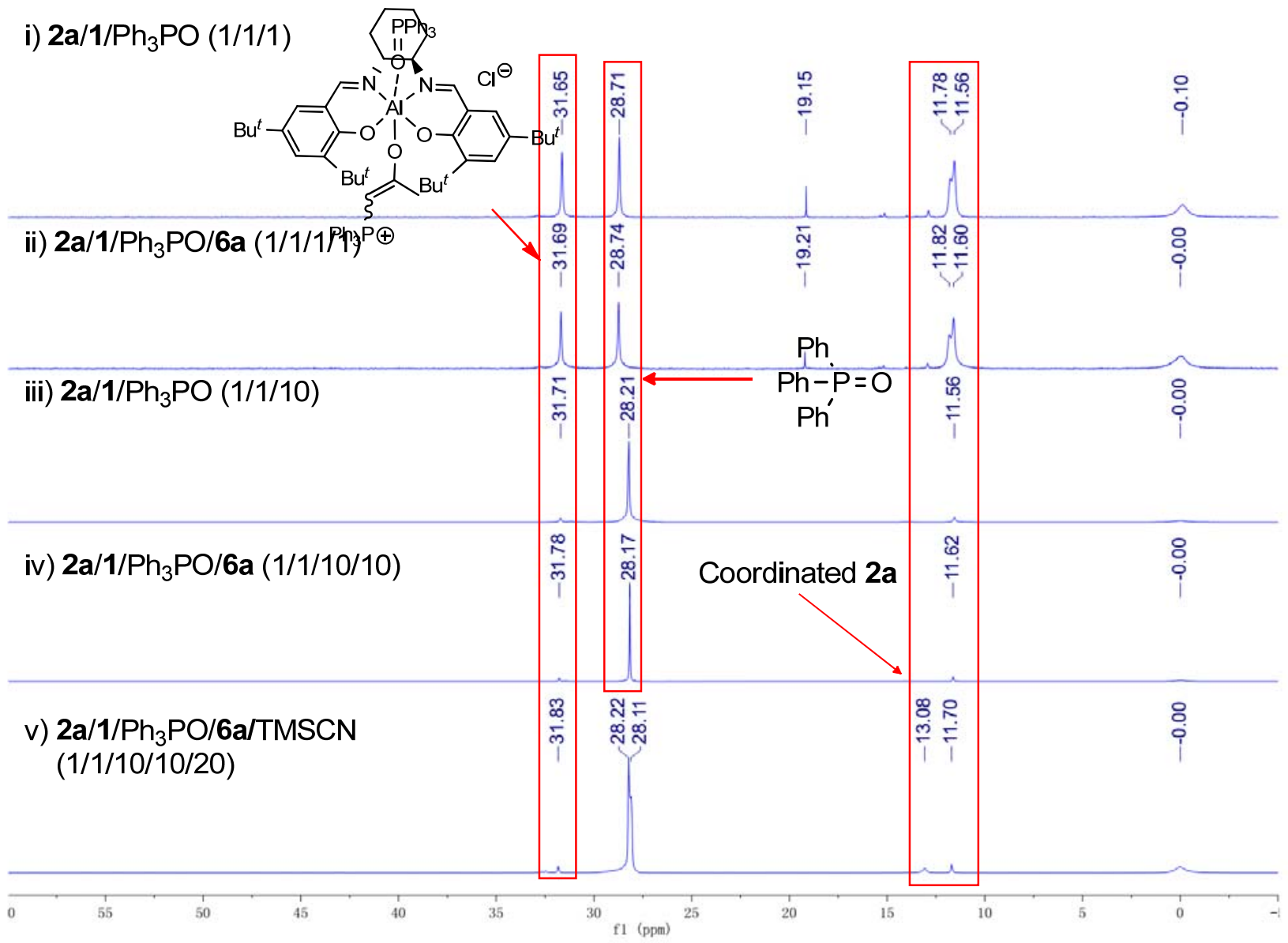

To probe the possible structure of intermediate chiral catalyst-substrate enone $\mathbf{6 a}$ complex, ${ }^{31} \mathrm{P}$ NMR analysis of a mixture of chiral complex $(R, R)-\mathbf{1}$, phosphorane $\mathbf{2 a}, \mathrm{Ph}_{3} \mathrm{PO}$ and enone $\mathbf{6 a}$ (with a ratio of $1 / 1 / 1 / 1)$ gave a similar spectrum to that of $\mathbf{2 a} /(R, R)-\mathbf{1} / \mathrm{Ph}_{3} \mathrm{PO}(1 / 1 / 1)$ (i vs ii, Figure $\left.\mathrm{S} 4\right)$. The spectrum of ii showed obvious signal of bounded $\mathrm{Ph}_{3} \mathrm{PO}$ at $\delta 31.69 \mathrm{ppm}$, suggesting part of $\mathrm{Ph}_{3} \mathrm{PO}$ still coordinate to the chiral aluminum complex even in the presence of substrate 6a. Similar results were observed when increasing the amount of $\mathrm{Ph}_{3} \mathrm{PO}$ and 6a to ten times relative to chiral complex $(R, R)-\mathbf{1}$ and phosphorane $\mathbf{2 a}$, close to the real reaction condition (iii vs iv). These results suggested that the coordination of $\mathrm{Ph}_{3} \mathrm{PO}$ and enone 6a to the reactive chiral aluminum complex was in a balance. We further conducted the ${ }^{31} \mathrm{P}$ NMR analysis of the reaction mixture of 2a/ $(R, R)-\mathbf{1} / \mathrm{Ph}_{3} \mathrm{PO} / \mathbf{6 a} / \mathrm{TMSCN}$ (with a ratio of $1 / 1 / 10 / 10 / 20$ ) in $\mathrm{CD}_{2} \mathrm{Cl}_{2}$. Besides the peaks that have been mentioned above, two new peaks appeared at 28.11 and $13.08 \mathrm{ppm}$ was developed in the resulting spectrum (v, Figure S4). The peak at $28.11 \mathrm{ppm}$ might be correlated to the reactive 
isocyanide species $\mathrm{Ph}_{3} \mathrm{P}(\mathrm{OTMS})\left(\mathrm{N}=\mathrm{C}\right.$ : ) that derived from the activation of $\mathrm{TMSCN}$ by $\mathrm{Ph}_{3} \mathrm{PO}$, proposed by Corey and coworkers ${ }^{12}$. And the peak at 13.08 ppm might be correlated to a certain intermediate of the reaction that we have not yet confirm. Noticeably, there were still no free phosphorane $\mathbf{2 a}$ detected during the reaction process, indicating the binding of phosphorane $\mathbf{2 a}$ to chiral complex $(R, R)-\mathbf{1}$ during the reaction process. The full ${ }^{31} \mathrm{P}$ NMR spectra of 1:1:1:1 and 10:10:1:1 mixture of $\mathrm{Ph}_{3} \mathrm{PO} / \mathbf{6} \mathbf{a} / \mathbf{2 a} /(R, R)-\mathbf{1}$ and 20:10:10:1:1 mixture of $\mathrm{TMSCN} / \mathrm{Ph}_{3} \mathrm{PO} / \mathbf{6} \mathbf{a} / \mathbf{2 a} /(R, R)-\mathbf{1}$ at different temperatures were shown in Figures S16-S18.

Based on the aforementioned variable temperature ${ }^{31} \mathrm{P}$ NMR experiments, together with fact that the MS analysis detected an important peak at $\mathrm{m} / \mathrm{z} 1061.6$ that was consistent with the complex of $(R, R)-\mathbf{1}$ and phosphorane $2 \mathbf{a}$ and enone $\mathbf{6 a},[\mathbf{2 a} /(R, R)-\mathbf{1} / \mathbf{6} \mathbf{a}-\mathrm{Cl}]^{+}$, the assumed chiral catalyst and key intermediate were proposed as follows:
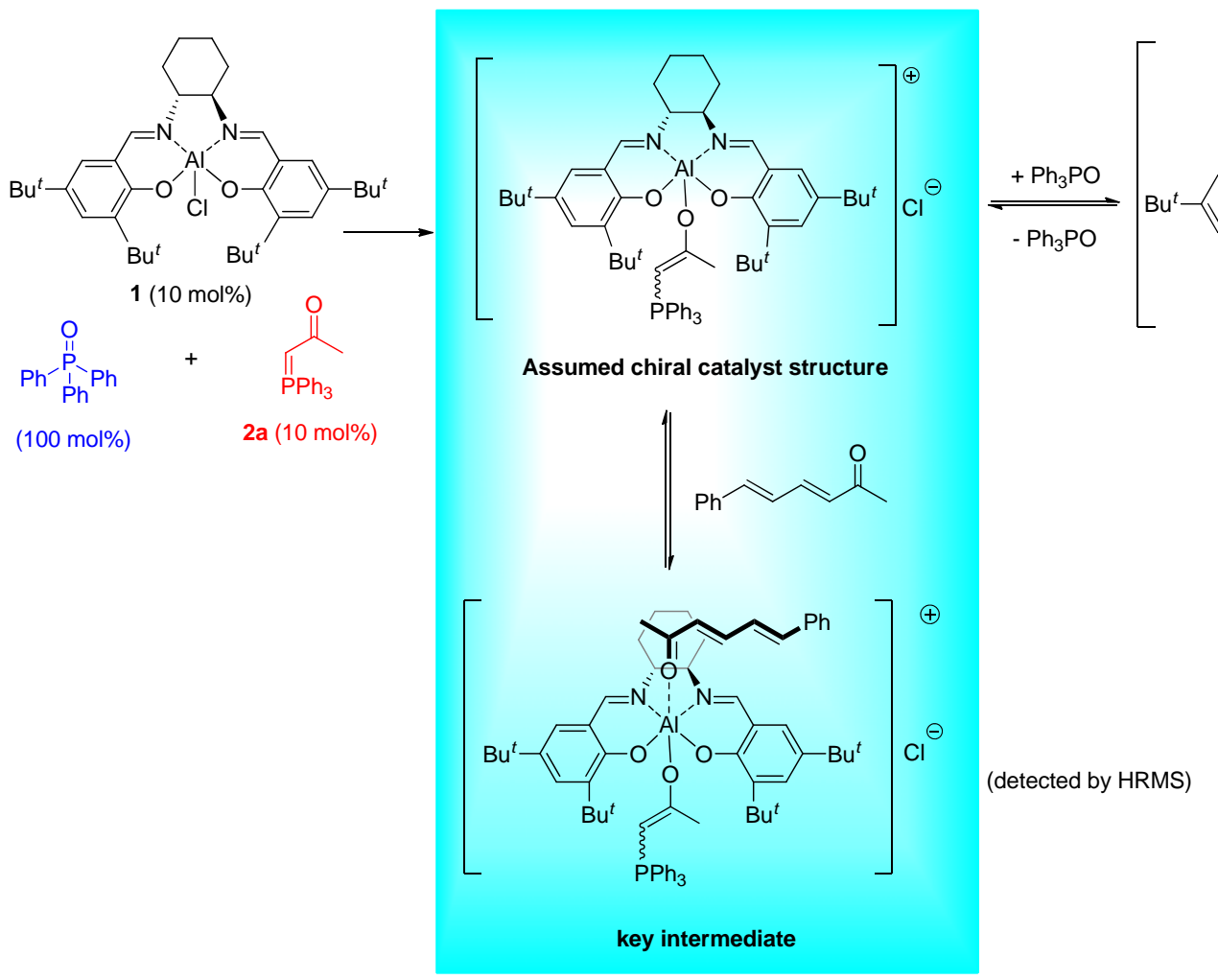
Figure S5. Effect of temperature on the ${ }^{31} \mathrm{P}$ NMR spectrum of $\mathbf{2 a}$

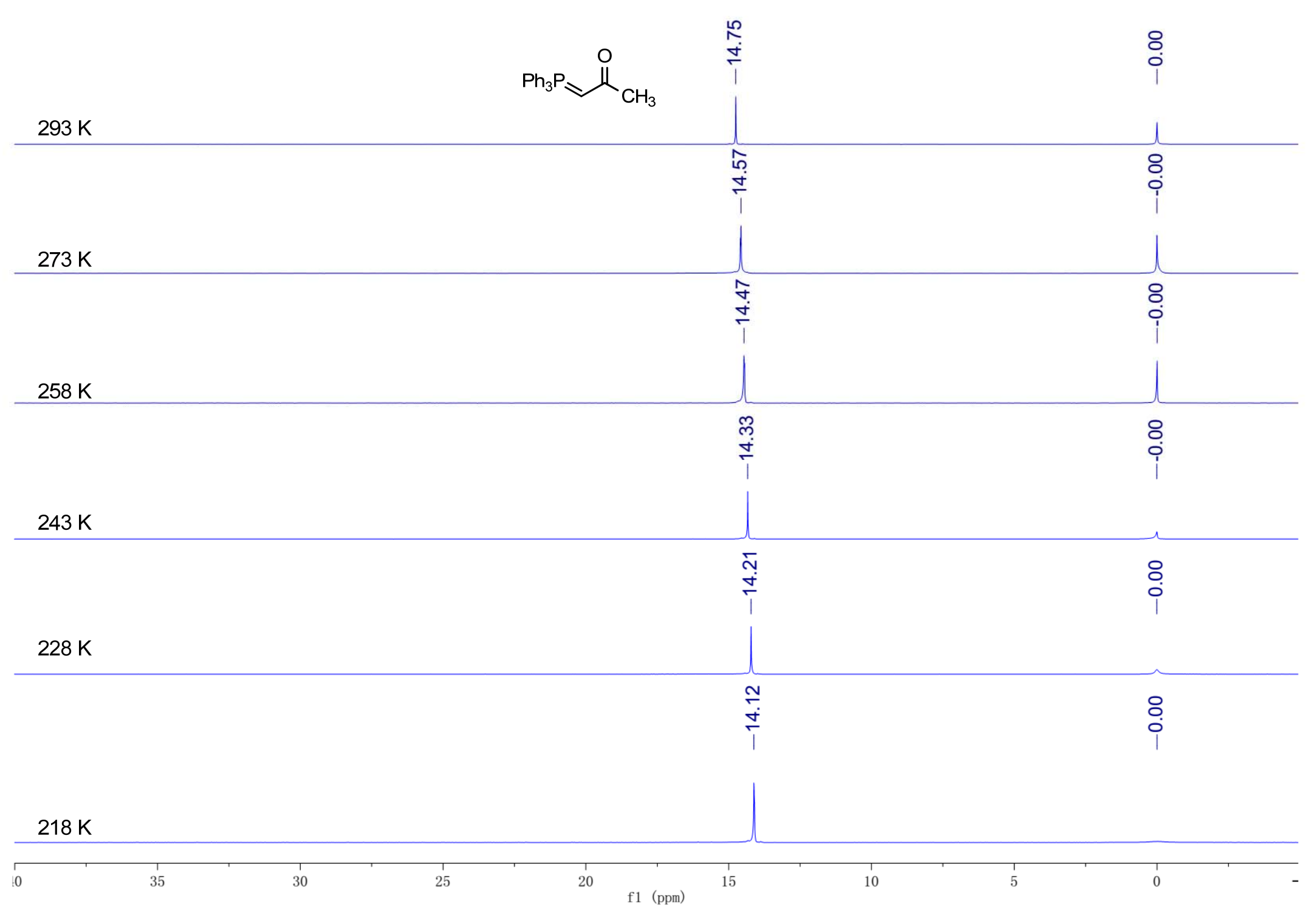


Figure S6. Effect of temperature on the ${ }^{31} \mathrm{P}$ NMR spectrum of a mixture of $(R, R)-\mathbf{1} / \mathbf{2 a}(1 / 1)$

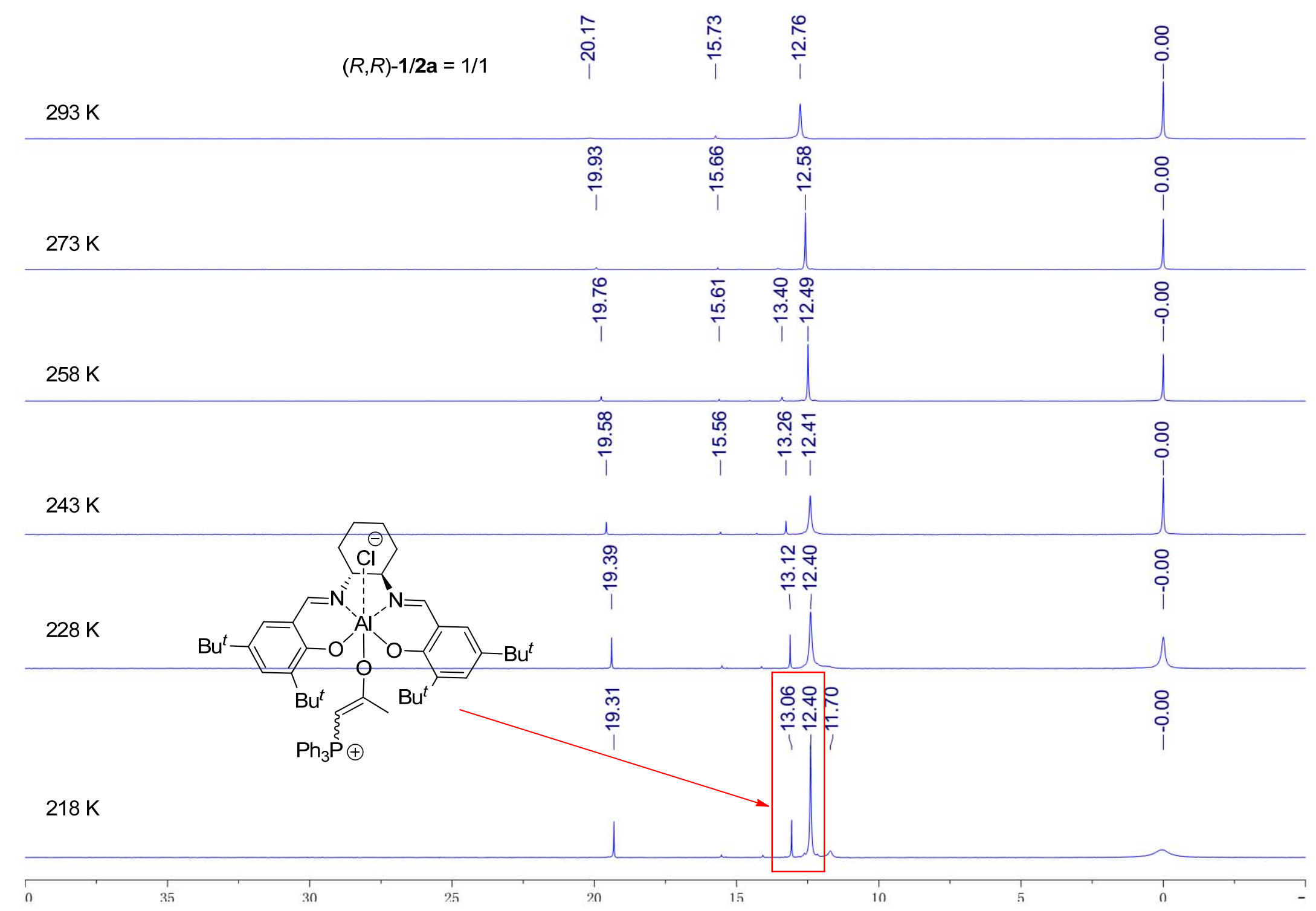


Figure S7. Effect of temperature on the ${ }^{31} \mathrm{P}$ NMR spectrum of a mixture of $(R, R)-\mathbf{1} / \mathbf{2 a}(1 / 2)$

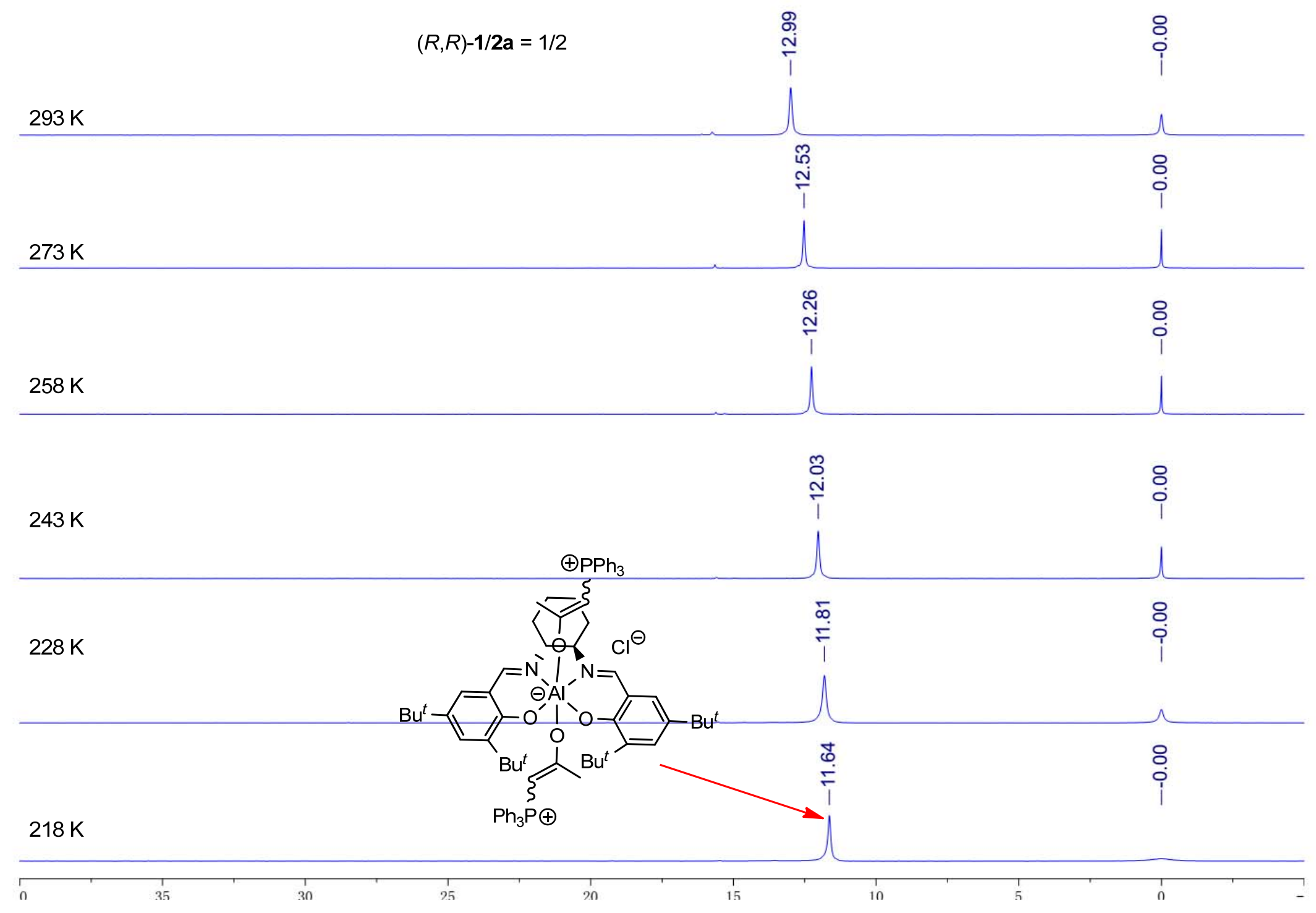


Figure S8. Effect of temperature on the ${ }^{31} \mathrm{P}$ NMR spectrum of a mixture of $(R, R)-\mathbf{1} / \mathbf{2 a}(1 / 4)$

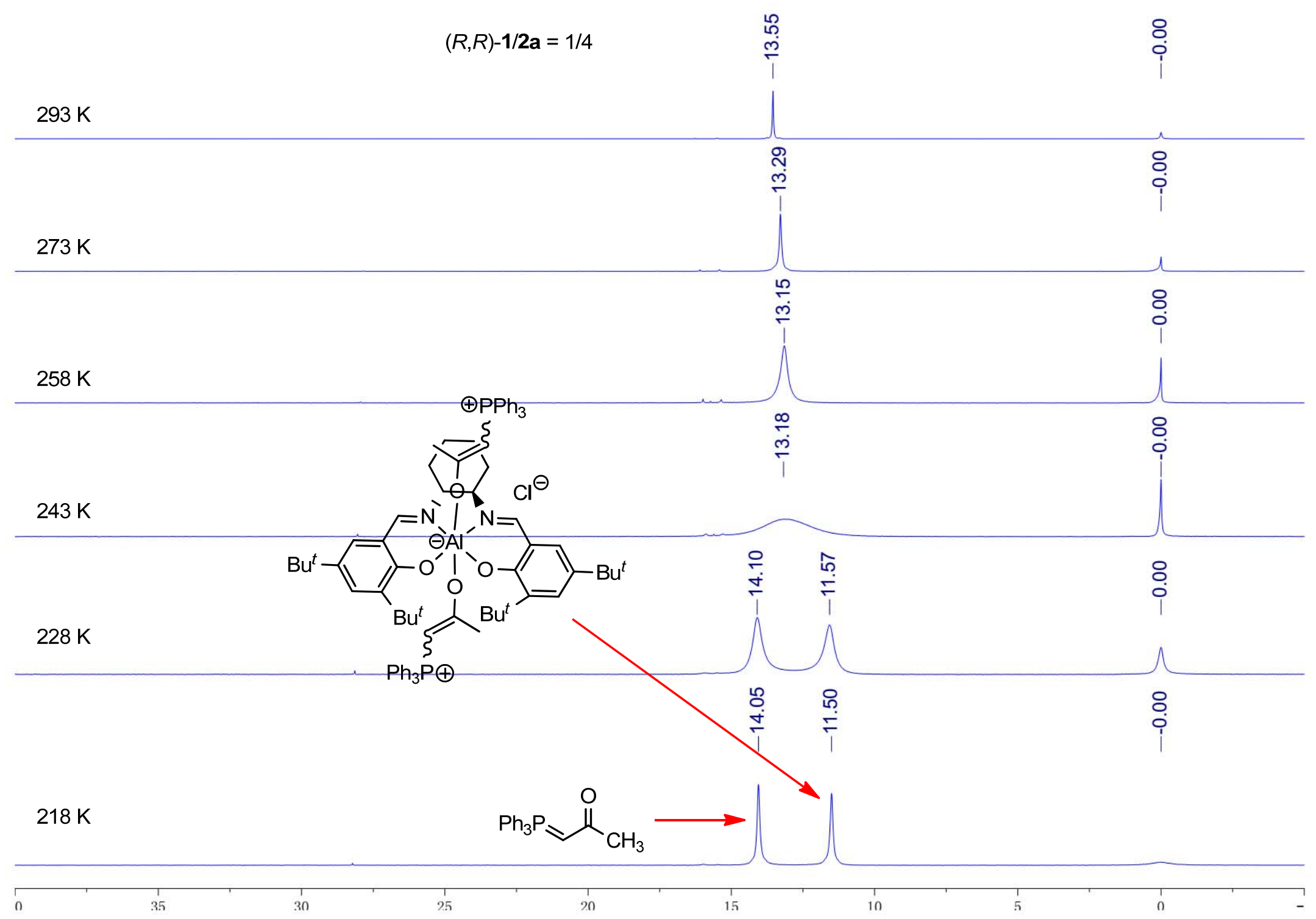


Figure S9. Effect of temperature on the ${ }^{31} \mathrm{P}$ NMR spectrum of $\mathrm{Ph}_{3} \mathrm{PO}$.

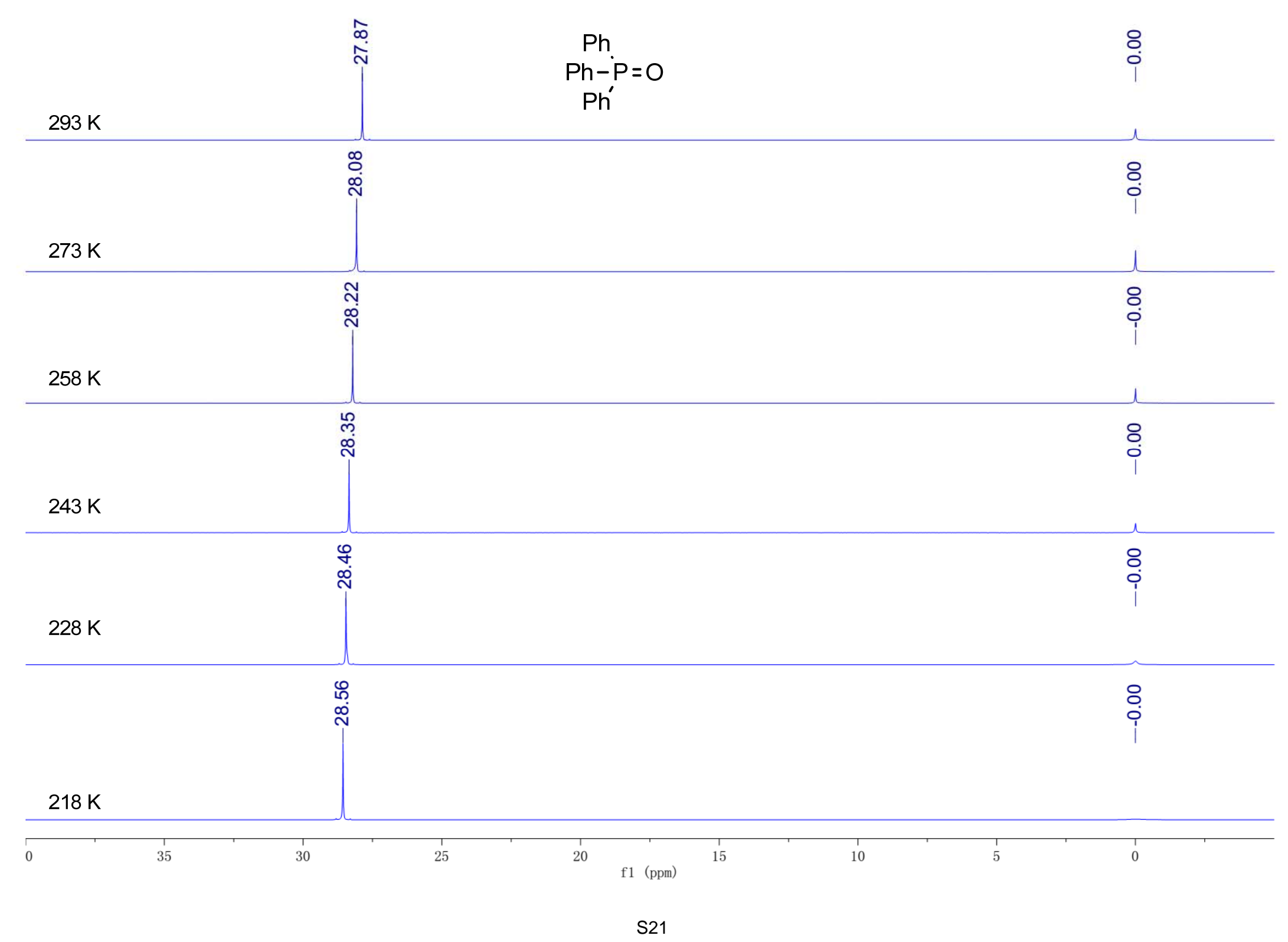


Figure S10. Effect of temperature on the ${ }^{31} \mathrm{P}-\mathrm{NMR}$ spectrum of a mixture of $(R, R)-\mathbf{1} / \mathrm{Ph}_{3} \mathrm{PO}(1 / 1)$

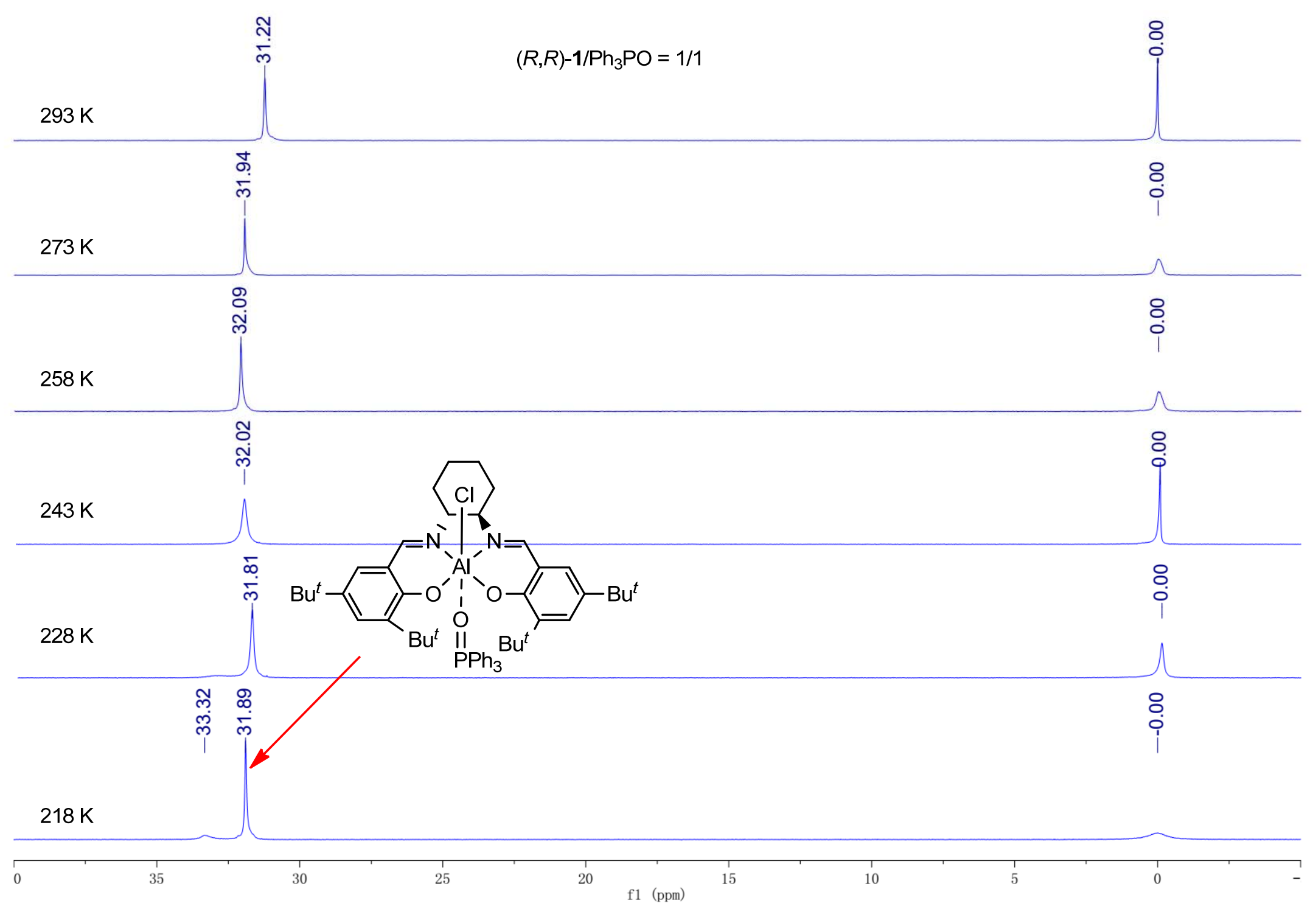


Figure S11. Effect of temperature on the ${ }^{31} \mathrm{P}-\mathrm{NMR}$ spectrum of a mixture of $(R, R)-\mathbf{1} / \mathrm{Ph}_{3} \mathrm{PO}(1 / 2)$

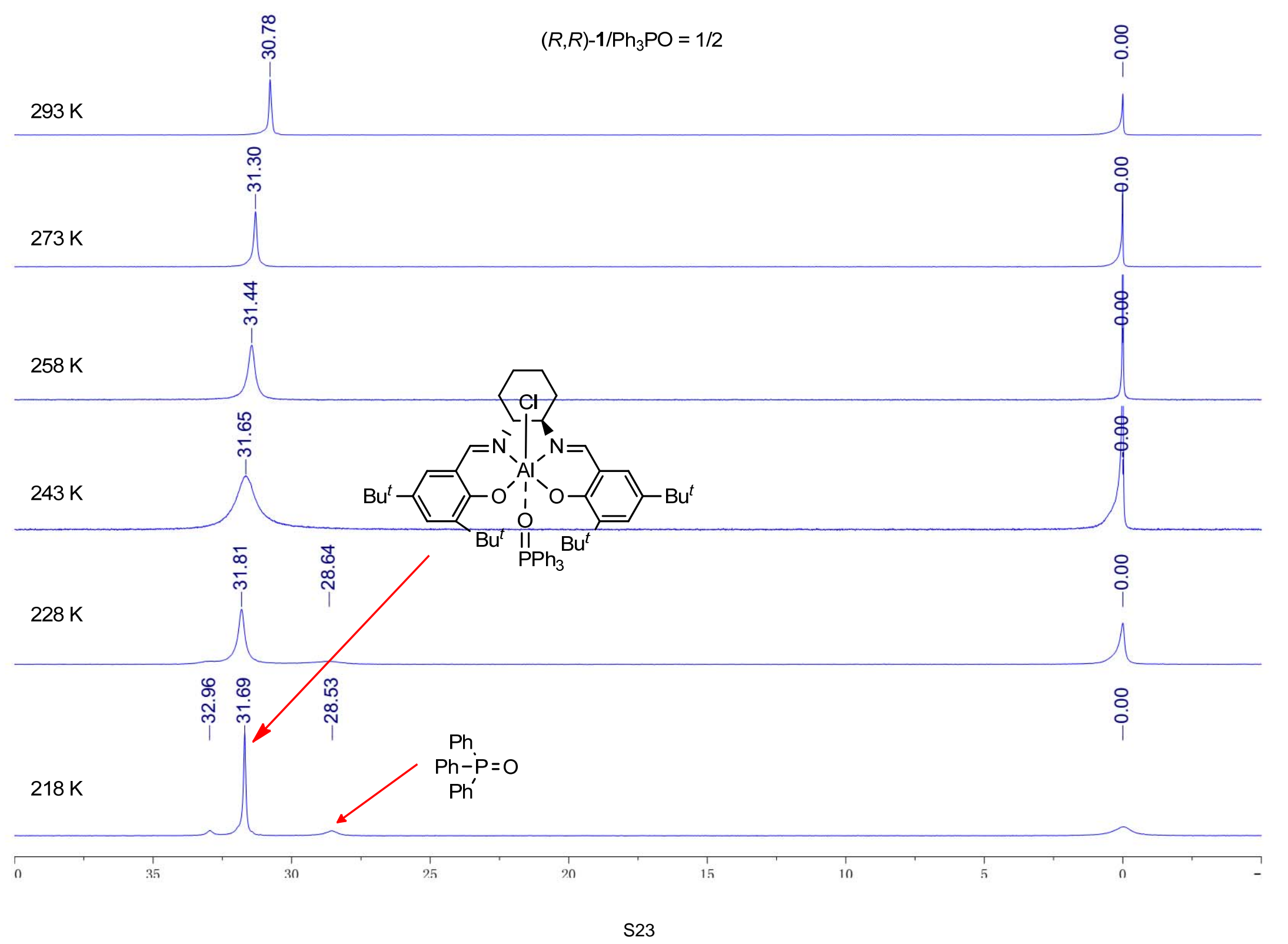


Figure S12. Effect of temperature on the ${ }^{31} \mathrm{P}-\mathrm{NMR}$ spectrum of a mixture of $(R, R)-\mathbf{1} / \mathrm{Ph}_{3} \mathrm{PO}(1 / 4)$

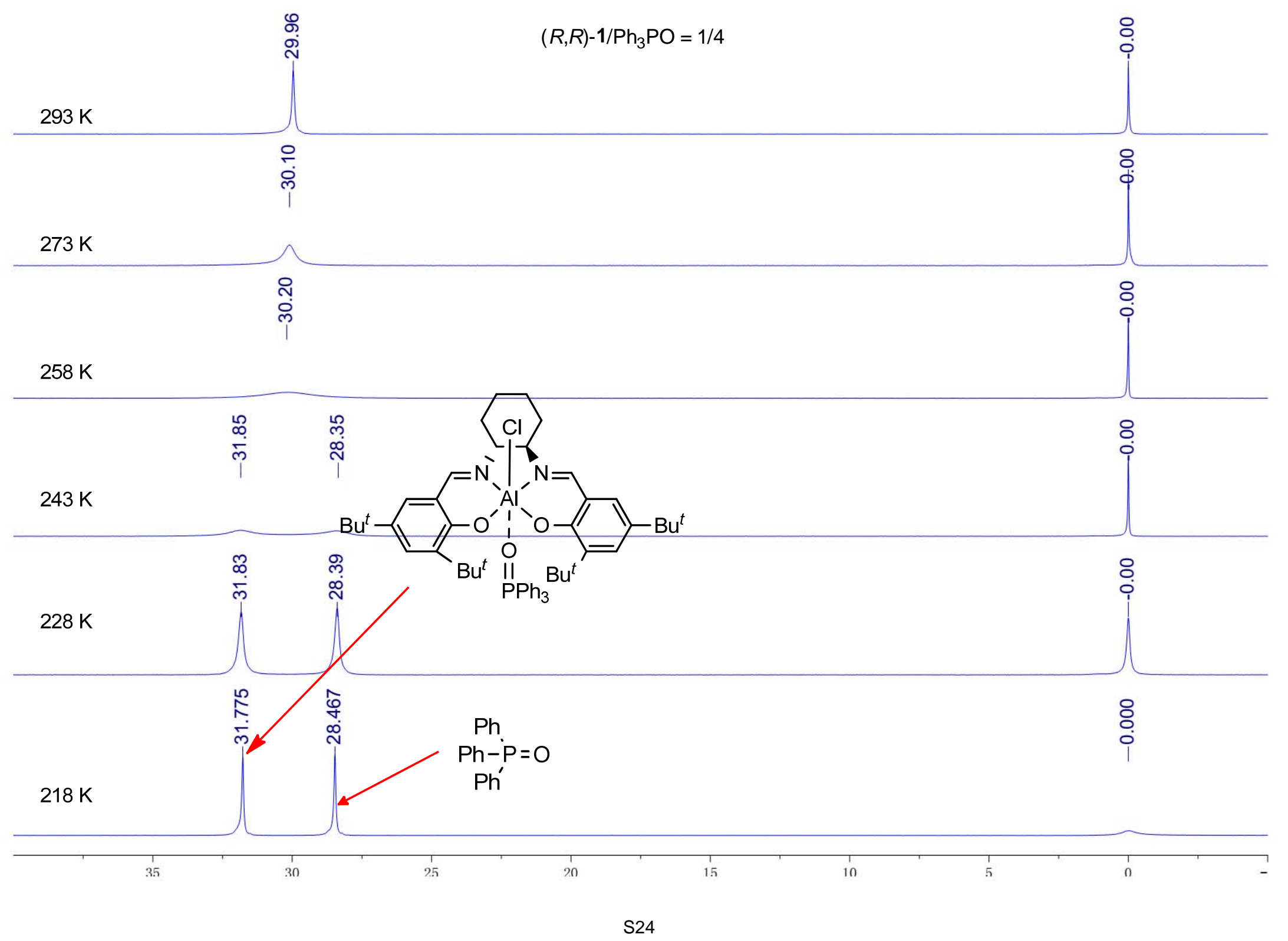


Figure S13. Effect of temperature on the ${ }^{31} \mathrm{P}-\mathrm{NMR}$ spectrum of a mixture of $(R, R)-\mathbf{1} / \mathrm{Ph}_{3} \mathrm{PO}(1 / 10)$

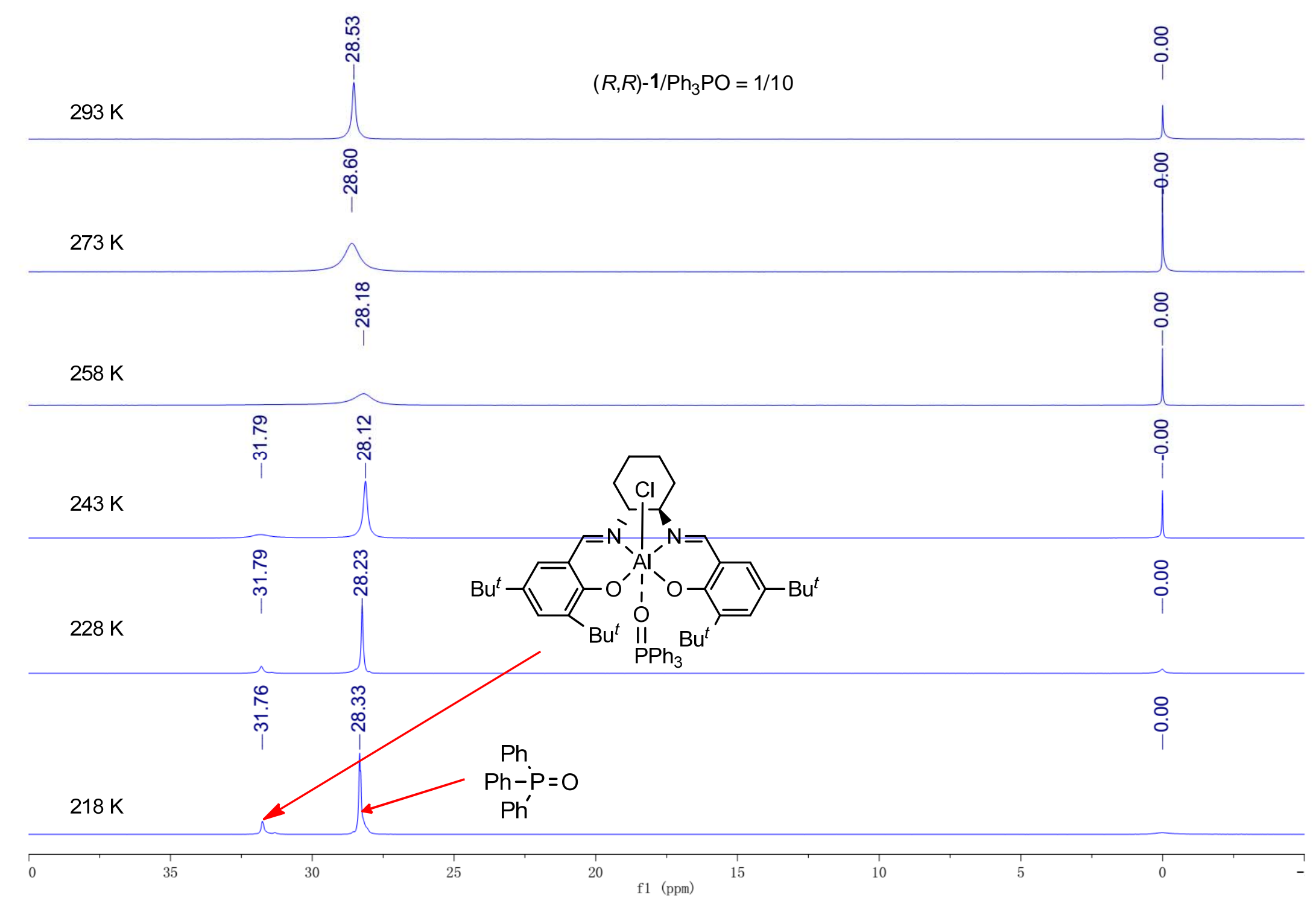


Figure S14. Effect of temperature on the ${ }^{31} \mathrm{P}-\mathrm{NMR}$ spectrum of a mixture of $(R, R)-\mathbf{1} / \mathrm{Ph}_{3} \mathrm{PO} / \mathbf{2 a}(1 / 1 / 1)$

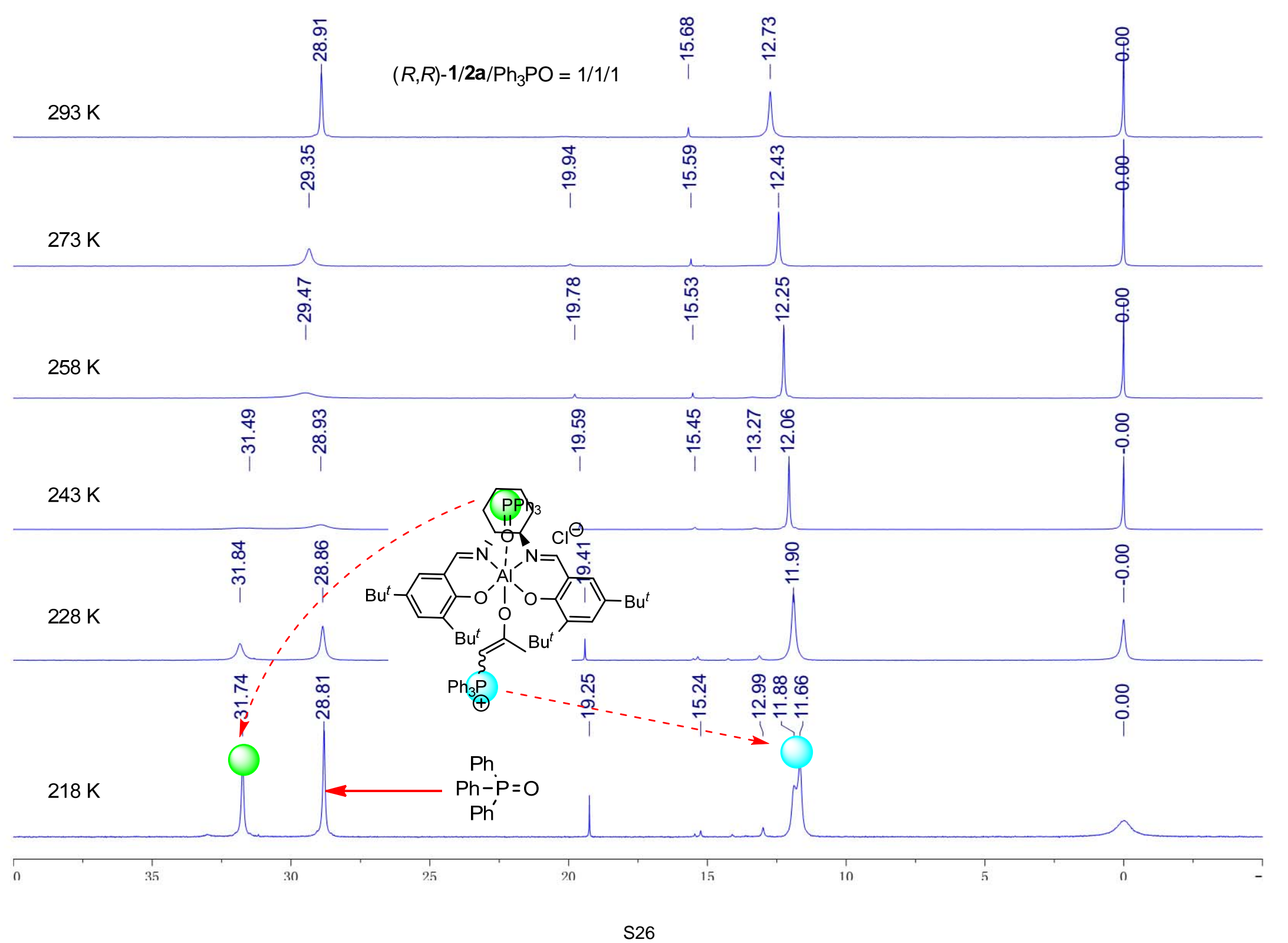


Figure S15. Effect of temperature on the ${ }^{31} \mathrm{P}-\mathrm{NMR}$ spectrum of a mixture of $(R, R)-\mathbf{1} / \mathbf{2} \mathbf{a} / \mathrm{Ph}_{3} \mathrm{PO}(1 / 1 / 10)$

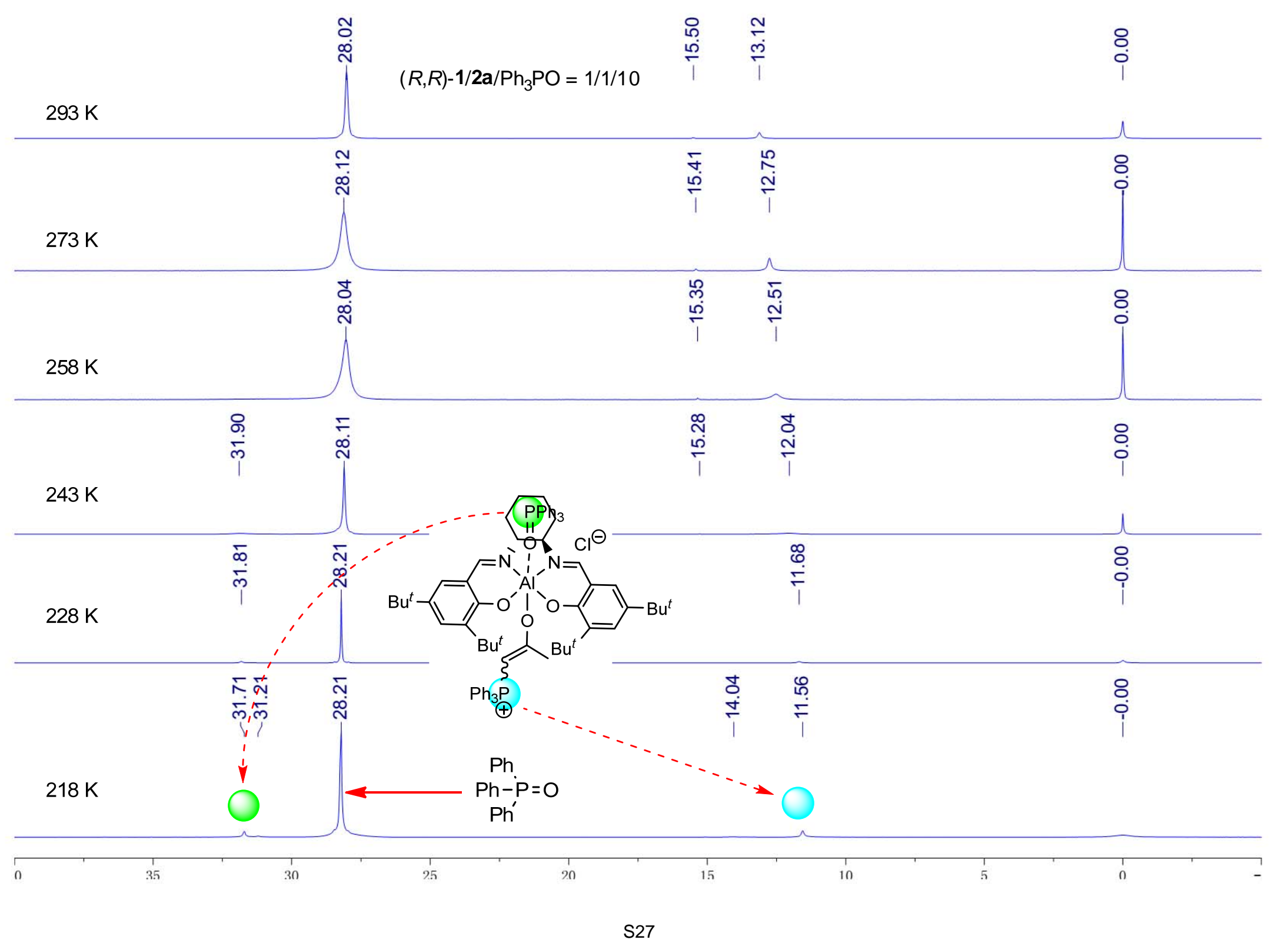


Figure S16. Effect of temperature on the ${ }^{31} \mathrm{P}-\mathrm{NMR}$ spectrum of a mixture of $(R, R)-\mathbf{1} / \mathrm{Ph}_{3} \mathrm{PO} / \mathbf{2 a} / \mathbf{6 a}(1 / 1 / 1 / 1)$

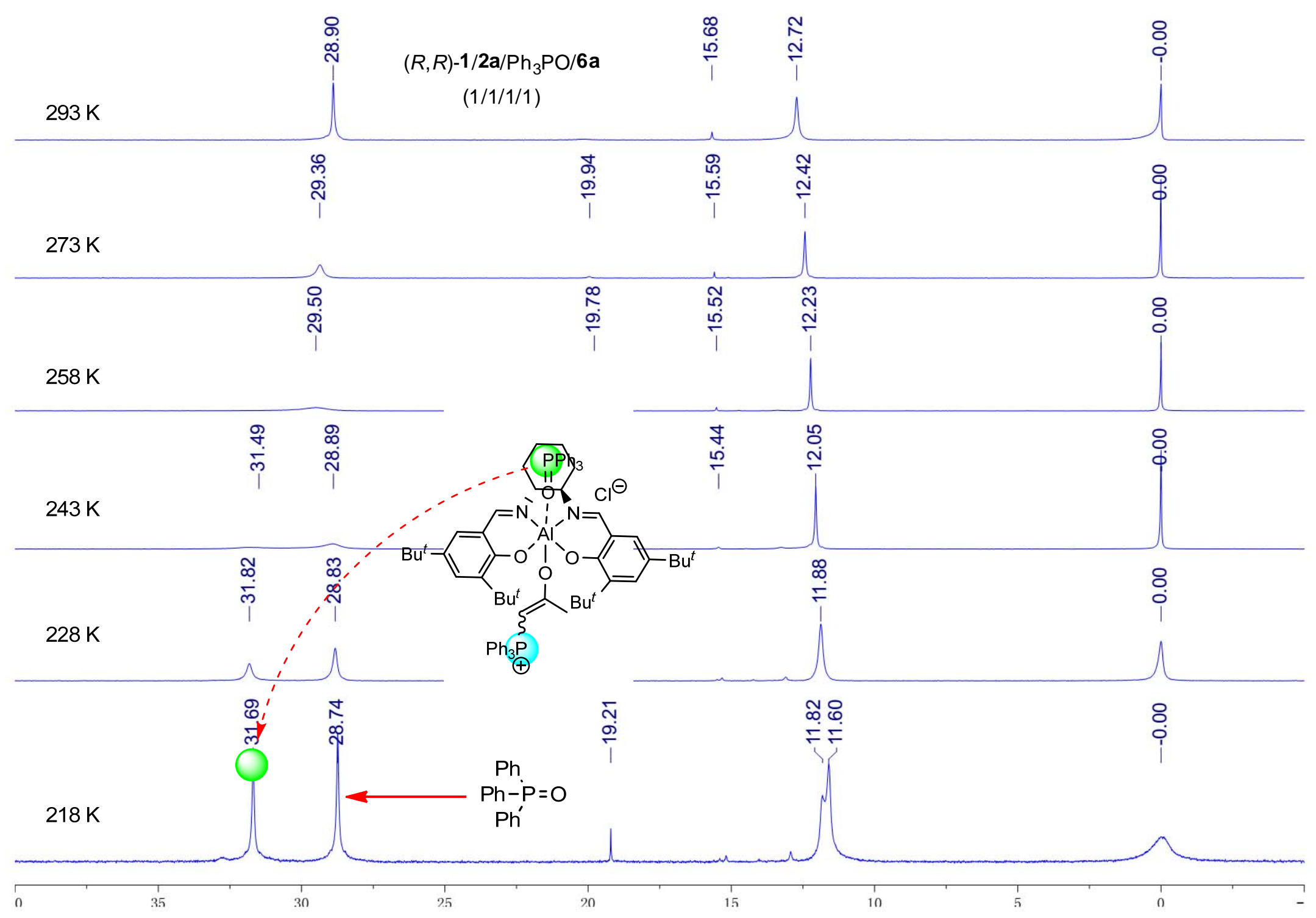


Figure S17. Effect of temperature on the ${ }^{31} \mathrm{P}-\mathrm{NMR}$ spectrum of a mixture of $(R, R)-\mathbf{1} / \mathbf{2} \mathbf{a} / \mathrm{Ph}_{3} \mathrm{PO} / \mathbf{6 a}=1 / 1 / 10 / 10$

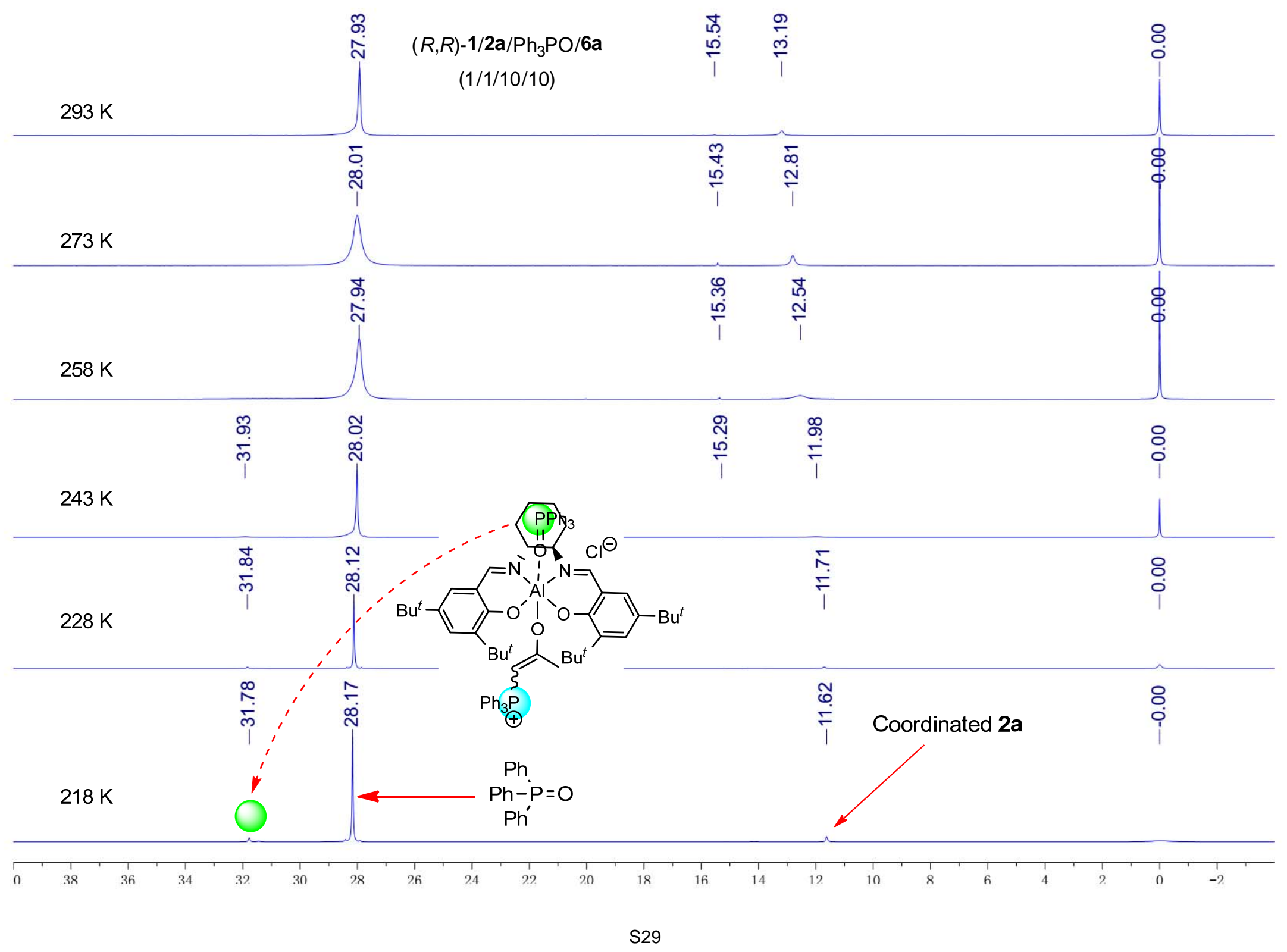


Figure S18. Effect of temperature on the ${ }^{31} \mathrm{P}-\mathrm{NMR}$ spectrum of a mixture of $(R, R)-\mathbf{1} / \mathbf{2} \mathbf{a} / \mathrm{Ph}_{3} \mathrm{PO} / \mathbf{6 a} / \mathrm{TMSCN}(1 / 10 / 10 / 10 / 20)$

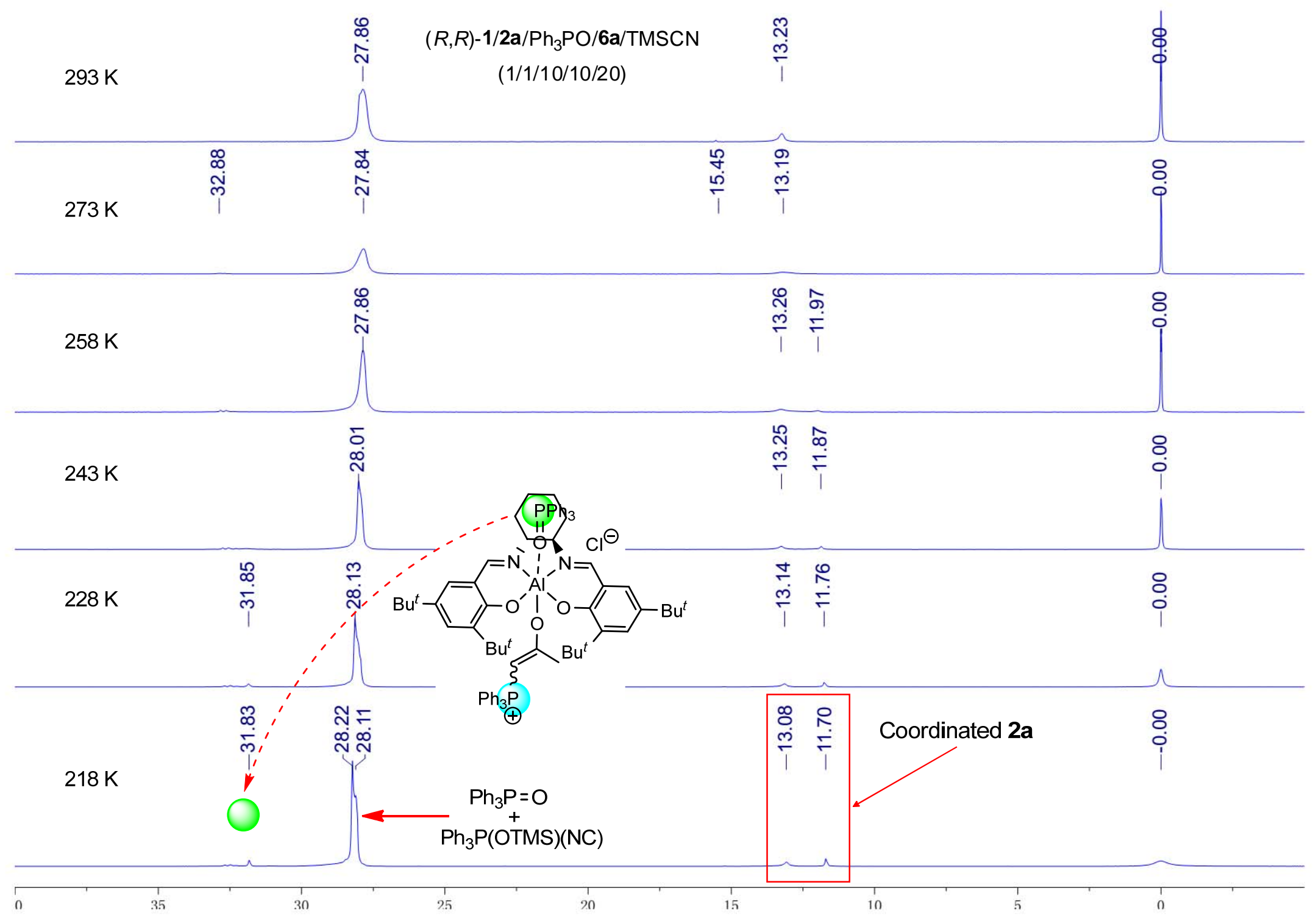




\section{2-3. Mass spectroscopy studies}

To get more information about the proposed intermediate, we further conducted MALDI-MS analysis (Instrument: Thermo Fisher Scientific LTQ FT Ultra; Operation Mode: MALDI_DHB) of the mixture of $10 \mathrm{~mol} \%$ of $(R, R)-\mathbf{1}, 10$ mol \% phosphorane $\mathbf{2 a}, 10$ mol \% $\mathrm{Ph}_{3} \mathrm{PO}$ and $100 \mathrm{~mol} \%$ enone 6a in $\mathrm{CH}_{2} \mathrm{Cl}_{2}$, which were shown in Figure $\mathrm{S} 19$ and its partially enlarged spectrum.

The signal $\left[(R, R)-\mathbf{1}+\mathrm{Ph}_{3} \mathrm{PO}-\mathrm{Cl}\right]^{+}$consistent with the complex of $(R, R)-\mathbf{1}$ and $\mathrm{Ph}_{3} \mathrm{PO}$ was observed at $m / z$ 849.5, supporting the coordination of $\mathrm{Ph}_{3} \mathrm{PO}$ to $(R, R)$-1. The signal at $\mathrm{m} / z$ 889.5 was consistent with the complex of $(R, R)-\mathbf{1}$ and phosphorane 2a, $[(R, R)-\mathbf{1}+\mathbf{2} \mathbf{a}-\mathrm{Cl}]^{+}$, suggesting the coordination of phosphorane $\mathbf{2 a}$ to $(R, R)-\mathbf{1}$. The characteristic peak $[\mathbf{2} \mathbf{a}+(R, R)-\mathbf{1}+\mathbf{6} \mathbf{a}-\mathrm{Cl}]^{+}$consistent with the complex of $(R, R)-\mathbf{1}$, phosphorane $\mathbf{2 a}$ and enone $\mathbf{6 a}$ was also detected at $\mathrm{m} / \mathrm{z}$ 1061.6, which suggested in the key intermediate, the activation of electrophilic enone $\mathbf{6 a}$ by a chiral cationic complex derived from the binding of phosphorane 2a to $(R, R)-\mathbf{1}$. In addition, the signal at $m / z 1207.6$ was correlating to the complex of $(R, R)-\mathbf{1}$ and two molecules of phosphorane $\mathbf{2 a},[\mathbf{2 a}+(R, R)-\mathbf{1}+\mathbf{2 a}-\mathrm{Cl}]$ ${ }^{+}$, which was in accordance with our NMR analysis that two molecules of phosphorane 2a might simultaneously coordinate to $(R, R)-\mathbf{1}$. The existence of these complexes were further confirmed by high resolution MS (Table S4)

Table S4. High resolution mass data for complexes derived from $(R, R)-\mathbf{1}$.

\begin{tabular}{|c|c|c|c|c|c|}
\hline Entry & Complex & $\begin{array}{c}\text { Ion's Elemental } \\
\text { Composition }\end{array}$ & $\begin{array}{c}\text { Theoretic } \\
\text { Mass }\end{array}$ & $\begin{array}{c}\text { Experimental } \\
\text { Mass }\end{array}$ & $\begin{array}{c}\text { Relative Error } \\
\text { (ppm) }\end{array}$ \\
\hline 1 & {$\left[\mathrm{Ph}_{3} \mathrm{PO}+(R, R)-\mathbf{1}-\mathrm{Cl}\right]^{+}$} & {$\left[\mathrm{C}_{54} \mathrm{H}_{67} \mathrm{AlN}_{2} \mathrm{O}_{3} \mathrm{P}\right]^{+}$} & 849.4699 & 849.4698 & -0.11 \\
\hline 2 & {$[\mathbf{2} \mathbf{a}+(R, R)-\mathbf{1}-\mathrm{Cl}]^{+}$} & {$\left[\mathrm{C}_{57} \mathrm{H}_{71} \mathrm{AlN}_{2} \mathrm{O}_{3} \mathrm{P}\right]^{+}$} & 889.5012 & 889.5004 & -0.94 \\
\hline 3 & {$[\mathbf{2} \mathbf{a}+(R, R)-\mathbf{1}+\mathbf{6} \mathbf{a}-\mathrm{Cl}]^{+}$} & {$\left[\mathrm{C}_{69} \mathrm{H}_{83} \mathrm{AlN}_{2} \mathrm{O}_{4} \mathrm{P}\right]^{+}$} & 1061.5900 & 1061.5891 & -0.87 \\
\hline 4 & {$[\mathbf{2} \mathbf{a}+(R, R)-\mathbf{1}+\mathbf{2} \mathbf{a}-\mathrm{Cl}]^{+}$} & {$\left[\mathrm{C}_{78} \mathrm{H}_{90} \mathrm{AlN}_{2} \mathrm{O}_{4} \mathrm{P}_{2}\right]^{+}$} & 1207.6186 & 1207.6194 & +0.70 \\
\hline
\end{tabular}


Figure S19. MALDI-MS spectrum analysis of 1:1:1:10 mixture of $\mathbf{2 a},(R, R)-\mathbf{1}, \mathrm{Ph}_{3} \mathrm{PO}$ and $\mathbf{6 a}$.

M150487 \#39 RT: $2.4726 \quad$ AV: 1 NL: $1.93 E 6$

T: FTMS + p MALDI Full ms [200.00-1500.00]

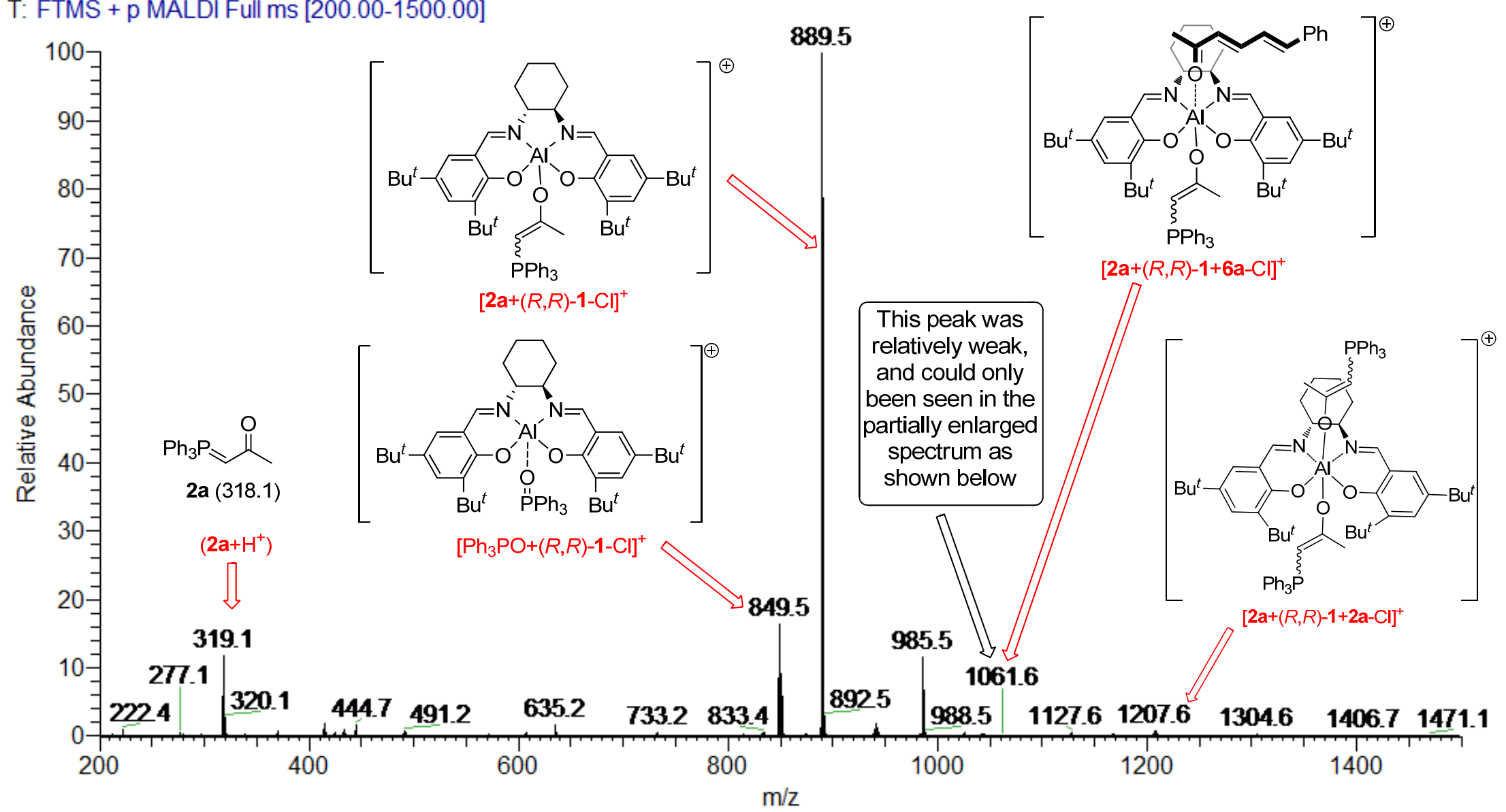




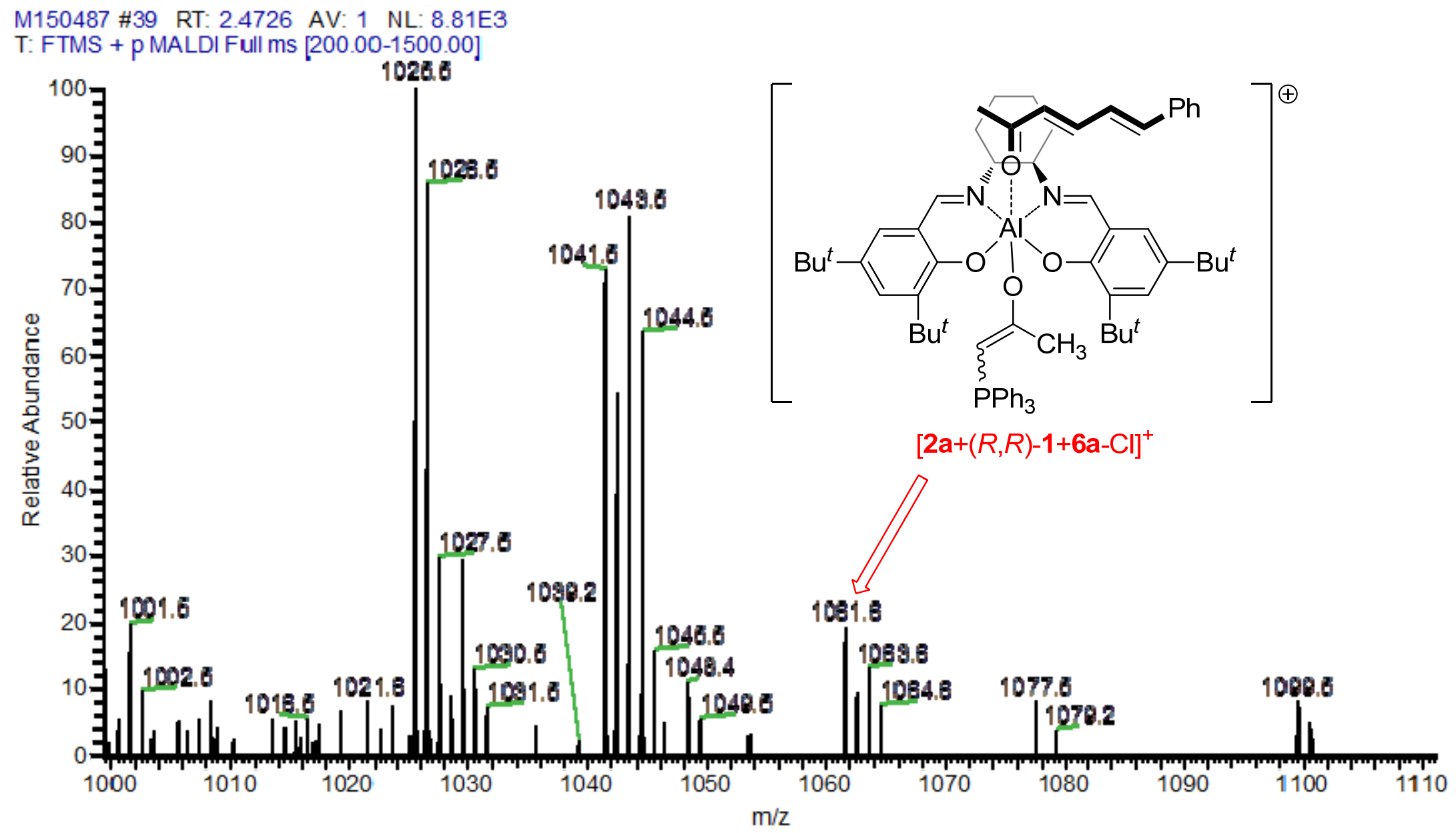




\section{2-4. In situ IR studies}

The purpose of React IR analysis was to further probe the binding of phosphorane 2a to chiral complex $(R, R)-\mathbf{1}$, and most importantly, the activation of TMSCN by $\mathrm{Ph}_{3} \mathrm{PO}$ during the reaction process.

General procedure: All the in situ IR studies were conducted at room temperature using an apparatus shown right, in which ReactIR 45m (by METTLER TOLEDO) and its fiber probe were used. After setting up the apparatus, $4 \mathrm{~mL} \mathrm{CH}_{2} \mathrm{Cl}_{2}$ was first added into the Schlenk tube and balanced for $5 \mathrm{~min}$ before slowly adding the corresponding compounds for in situ IR study.

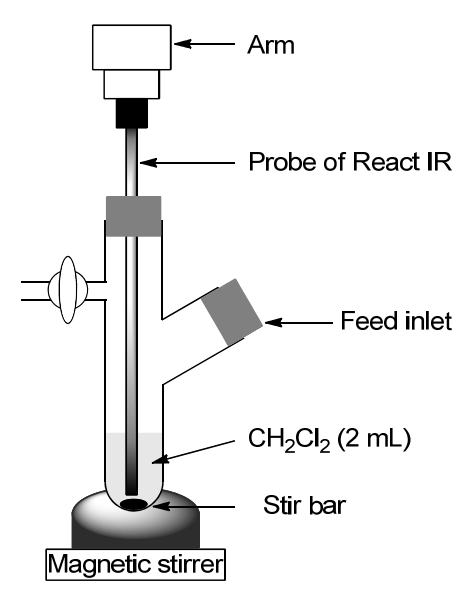

Apparatus for React IR experiment

\section{2-4-1. In situ study of the coordination of 2 a to $(R, R)-1$}

It has been reported that the $C$-coordination of phosphorane caused an increase in $v(\mathrm{CO})$, while the $O$-coordination resulted in a decrease in $v(\mathrm{CO}) .{ }^{15}$ For example, while the $v(\mathrm{CO})$ of free $\mathbf{2 a}$ located at $1539 \mathrm{~cm}^{-1}$, the $v(\mathrm{CO})$ of $C$-coordinated $2 \mathbf{a}$ located at $1582 \mathrm{~cm}^{-1}$ and that of $O$-coordinated $2 \mathrm{a}$ at $1509 \mathrm{~cm}^{-1} v(\mathrm{CO})$, according to reported data for phosphorane derived metal complexes. ${ }^{13 \mathrm{a}}$ So we tried in situ IR analysis to get more information on the binding of $\mathbf{2 a}$ to complex $(R, R)-\mathbf{1}$.

Figure S20. IR spectra of $\mathbf{2 a} /(R, R)-\mathbf{1}$ and $\mathbf{2 b} /(R, R)-\mathbf{1}$.
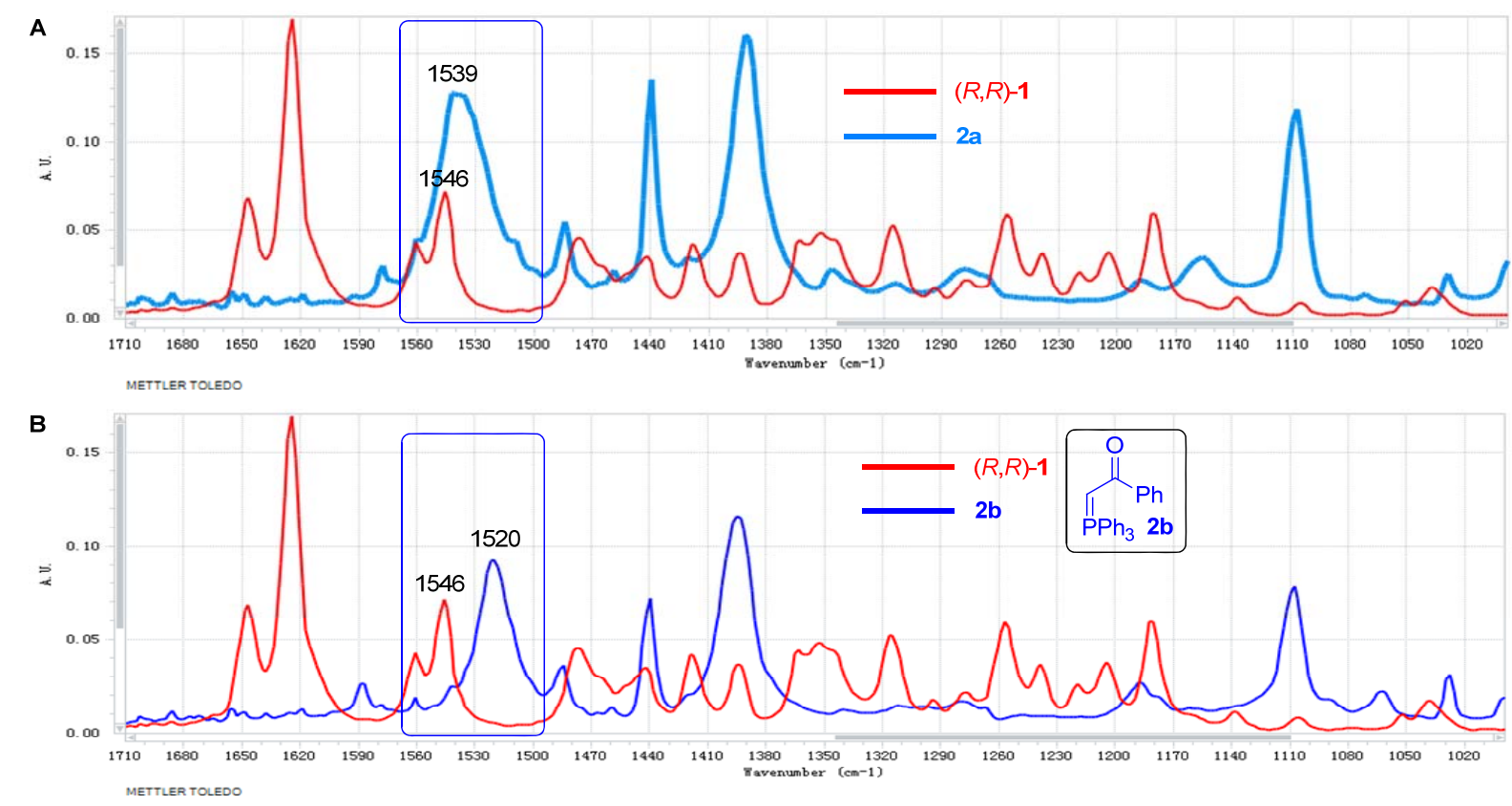

Unfortunately, as shown in A of Figure S20, the characteristic peak of the carbonyl group of 
phosphorane 2a overlapped with the signals of complex $(R, R)-1$ in the region of around $1540 \mathrm{~cm}^{-1}$, making it hard to monitor the change. After several trials, we found that the $v(\mathrm{CO})$ of phosphorane $\mathbf{2 b}$ $\left(1520 \mathrm{~cm}^{-1}\right)$ could well fit into one gap of the IR spectrum of $(R, R)-\mathbf{1}(\mathrm{B})$, so phosphorane $\mathbf{2 b}$ was employed in the following in situ IR study. The portion-wise addition of complex $(R, R)-\mathbf{1}$ into a solution of $\mathbf{2} \mathbf{b}$ resulted in a spectrum as shown in A of Figure S21. With the growing of the signal of $(R, R)-\mathbf{1}$ at $1546 \mathrm{~cm}^{-1}$, a decline of the characteristic peak of the carbonyl group of $\mathbf{2 b}$ at $1520 \mathrm{~cm}^{-1}$ was observed, which suggested the binding of $\mathbf{2} \mathbf{b}$ and $(R, R)-\mathbf{1}$. Unfortunately, no more useful information was obtained due to the complexity of the whole system. As shown in the full spectrum (B, Figure $\mathrm{S} 21$ ), when comparing the spectrum of free $\mathbf{2 b}$ (green) with that of $1: 1$ complex of $\mathbf{2} \mathbf{b} /(R, R)-\mathbf{1}$ (red), it was hard to get the information on how the $v(\mathrm{CO})$ shifted.

Figure S21. A) React IR study of the binding of $\mathbf{2 b}$ to $(R, R)-\mathbf{1}$; B) Full spectrum of $\mathbf{2} \mathbf{b}, \mathbf{2} \mathbf{b} /(R, R)-\mathbf{1}$ and $(R, R)-1$.

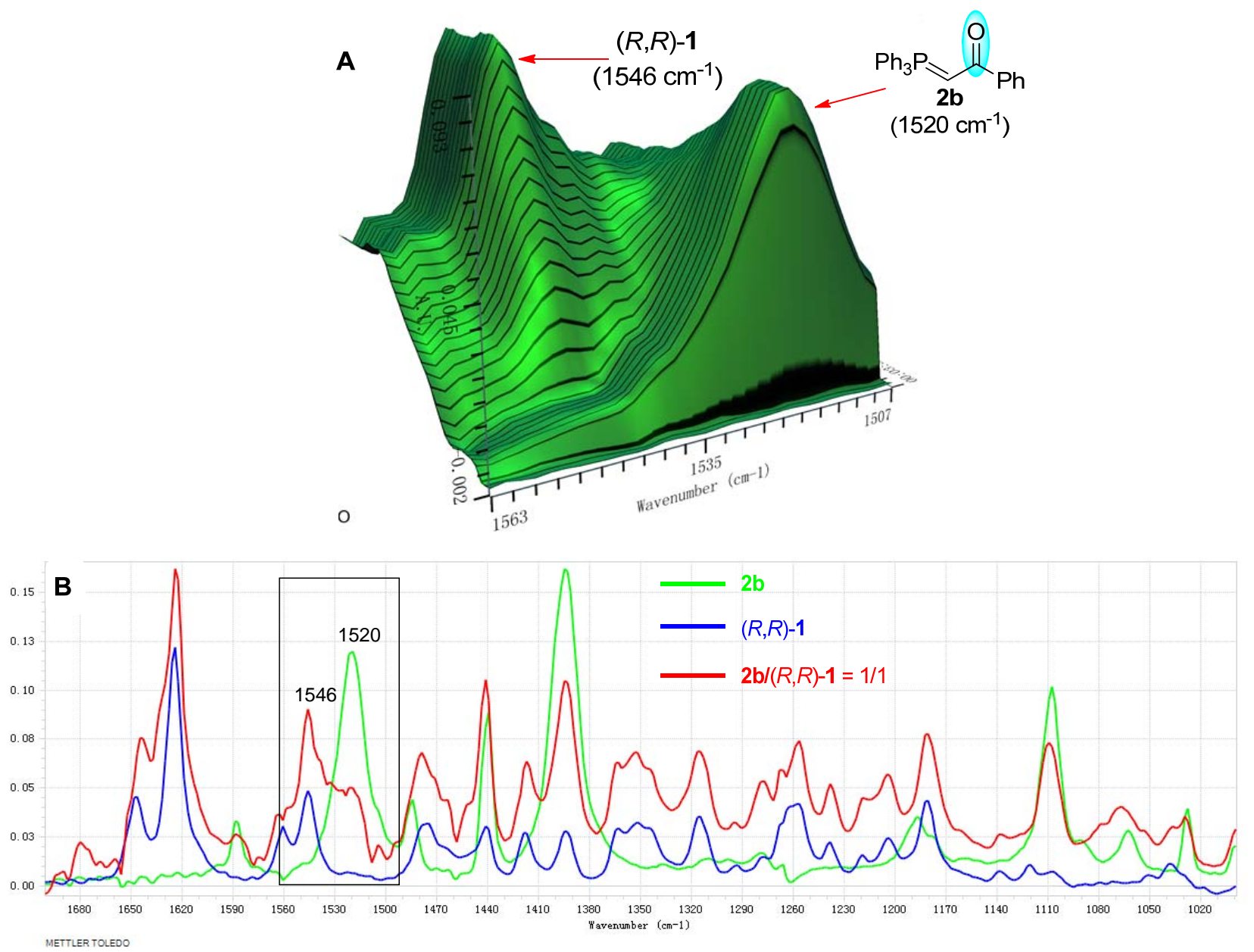




\section{2-4-2. IR Study the activation of $\mathrm{Ph}_{3} \mathrm{PO}$ to TMSCN}

As introduced previously, Corey and Ryn have reported the IR analysis of a mixture of TMSCN and $\mathrm{Ph}_{3}$ PO. A new -NC stretching band at $2072 \mathrm{~cm}^{-1}$, different from that of TMSCN $\left(2192 \mathrm{~cm}^{-1}\right)$ and TMSNC $\left(2095 \mathrm{~cm}^{-1}\right)$, suggested the formation of reactive isocyanide species $\mathrm{Ph}_{3} \mathrm{P}(\mathrm{OTMS})(\mathrm{N}=\mathrm{C}:$ ). Our React IR analysis was also in agreement with their finding, as the slow addition of $\mathrm{Ph}_{3} \mathrm{PO}$ to a solution of TMSCN in anhydrous $\mathrm{CH}_{2} \mathrm{Cl}_{2}$ resulted in an obvious development of peak at $2074 \mathrm{~cm}^{-1}$, and a balance could be reached almost as soon as the addition finished (A of Figure S22). The full spectrum was shown in B of Figure S22. These results offered the opportunity for us to investigate whether the role of $\mathrm{Ph}_{3} \mathrm{PO}$ in our reaction system was also to activate TMSCN to form a more reactive isocyanide species $\mathrm{Ph}_{3} \mathrm{P}(\mathrm{OTMS})(\mathrm{N}=\mathrm{C}$ :).

Figure S22. A) React IR study of the reaction between TMSCN and $\mathrm{Ph}_{3} \mathrm{PO}$; B) Full spectrum of TMSCN, TMSCN/Ph 3 PO and $\mathrm{Ph}_{3} \mathrm{PO}$.
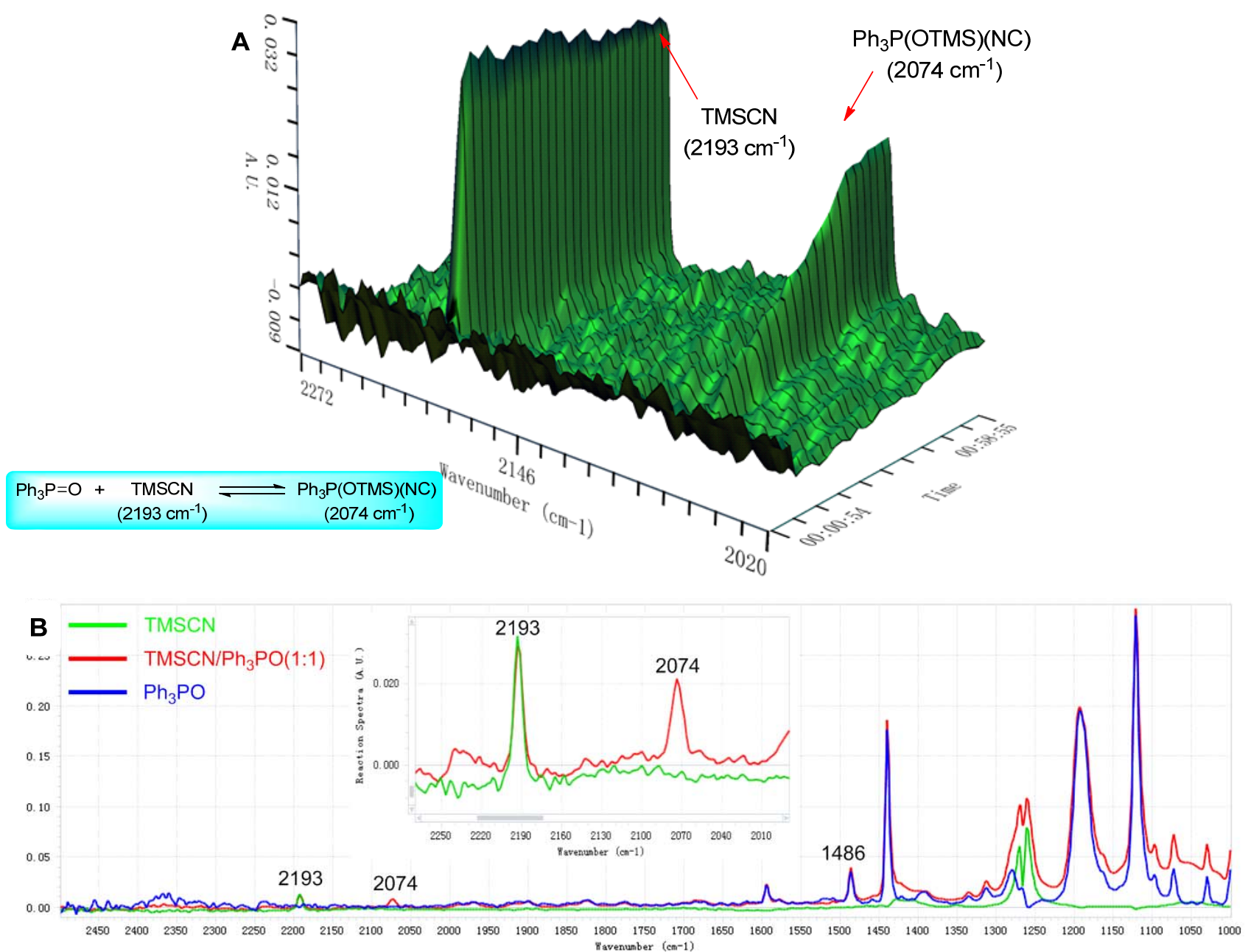


\section{2-4-3. IR Study of the whole reaction process}

To get clear observation about the change of the characteristic peak of the carbonyl group of ketone during the reaction process, the cyanosilylation of ketone 8f, mediated by 10 mol \% $(R, R)-\mathbf{1}$, $10 \mathrm{~mol} \%$ phosphorane $\mathbf{2 b}$ and $100 \mathrm{~mol}_{\%} \mathrm{Ph}_{3} \mathrm{PO}$, was chosen for React IR analysis after careful trials, as the $v(\mathrm{CO})$ of ketone $\mathbf{8 f}$ located far from other peaks. As shown in A of Figure S23, after the system reached a balance, $\mathbf{8 f}$ was added slowly to a solution of $(R, R)-\mathbf{1}, \mathbf{2 b}$ and $\mathrm{Ph}_{3} \mathrm{PO}$ in $\mathrm{CH}_{2} \mathrm{Cl}_{2}$, which could be seen from the growth of the $v(\mathrm{CO})$ of ketone $\mathbf{8 f}$ at $1716 \mathrm{~cm}^{-1}$. Then TMSCN was added in one portion to start the reaction. The immediate appearance of a peak at $2074 \mathrm{~cm}^{-1}$ clearly demonstrated the activation of TMSCN by $\mathrm{Ph}_{3} \mathrm{PO}$ to form the reactive $\mathrm{Ph}_{3} \mathrm{P}(\mathrm{OTMS})(\mathrm{N}=\mathrm{C}$ :) species.

Figure S23. A) React IR analysis of cyanosilylation of ketone 8f; B) Full spectrum at start and end of the cyanosilylation of $\mathbf{8 f}$.
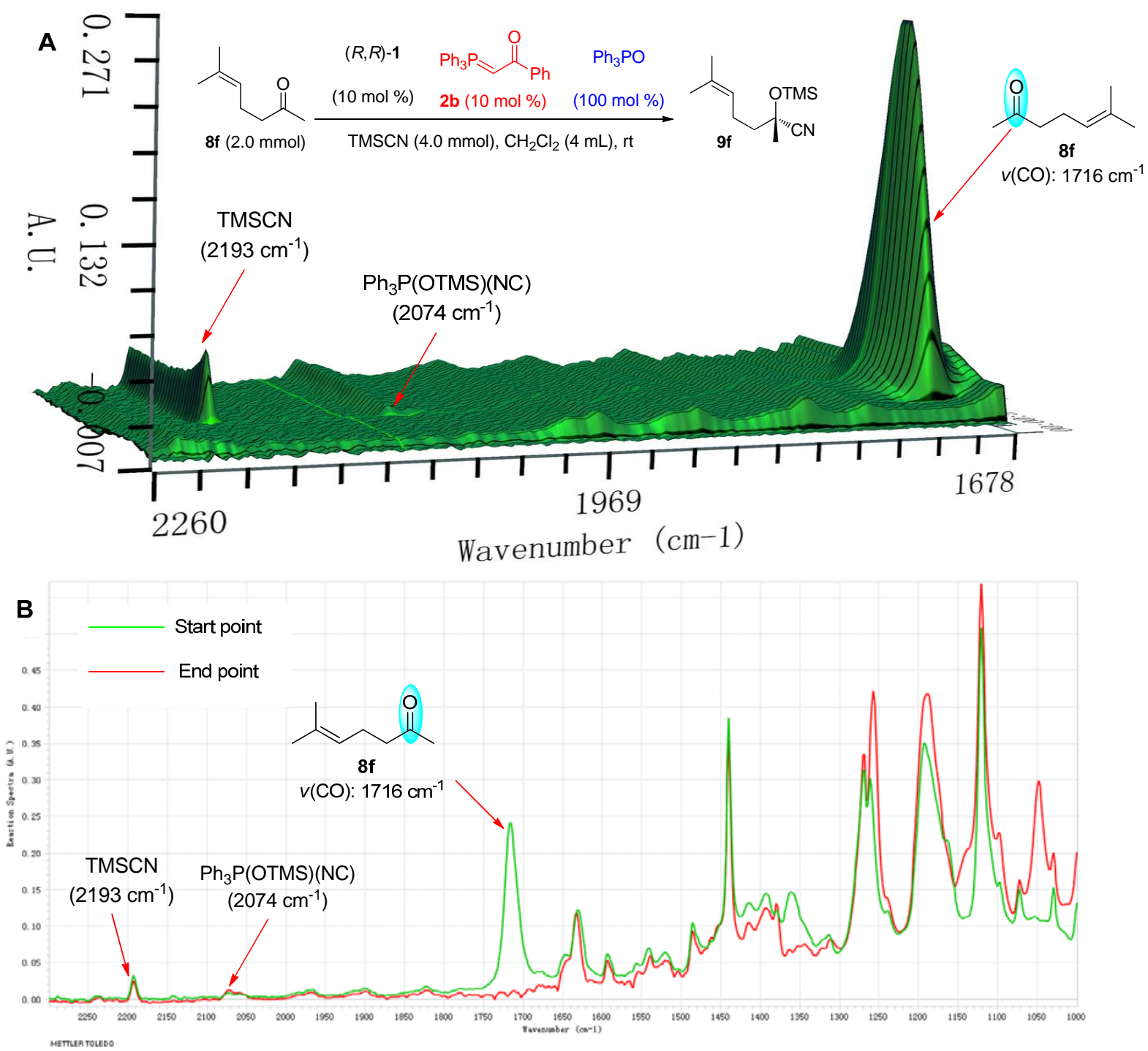
At room temperature, it took about half an hour for completion of the reaction, as estimated by the decreasing rate of the $v(\mathrm{CO})$ of $\mathbf{8 f}$ at $1716 \mathrm{~cm}^{-1}$. To probe the acceleration effect of $\mathrm{Ph}_{3} \mathrm{PO}$, we further conducted a control experiment with reversed addition (A, Figure S24). The reaction of $\mathbf{8 f}$ and TMSCN, mediated by $10 \mathrm{~mol} \%(R, R)-\mathbf{1}$ and $10 \mathrm{~mol} \% \mathbf{2} \mathbf{b}$, proceeded slowly at room temperature, as evidenced by the slowly decreasing of $v(\mathrm{CO})$ of $\mathbf{8 f}$, with less than $20 \%$ conversion after $0.5 \mathrm{~h}$. After $50 \mathrm{mmol} \% \mathrm{Ph}_{3} \mathrm{PO}$ was added in one portion (00:43:00), a new peak at $2074 \mathrm{~cm}^{-1}$ correlating to $\mathrm{Ph}_{3} \mathrm{P}(\mathrm{OTMS})(\mathrm{N}=\mathrm{C}$ :) was immediately developed, and the rate acceleration (from 00:43:00 to 01:08:00) was obvious judged by the change of $v(\mathrm{CO})$ of $\mathbf{8 f}$, although the concentration of substrates decreased. These results clearly supported the activation TMSCN by $\mathrm{Ph}_{3} \mathrm{PO}$. A more vivid description of this process could be seen from the surface spectrum (B).

Figure S24. A) The addition sequence for the confirmation of the acceleration effect of $\mathrm{Ph}_{3} \mathrm{PO} . \mathrm{B}$ ) Trend for the confirmation of the acceleration effect of $\mathrm{Ph}_{3} \mathrm{PO}$.

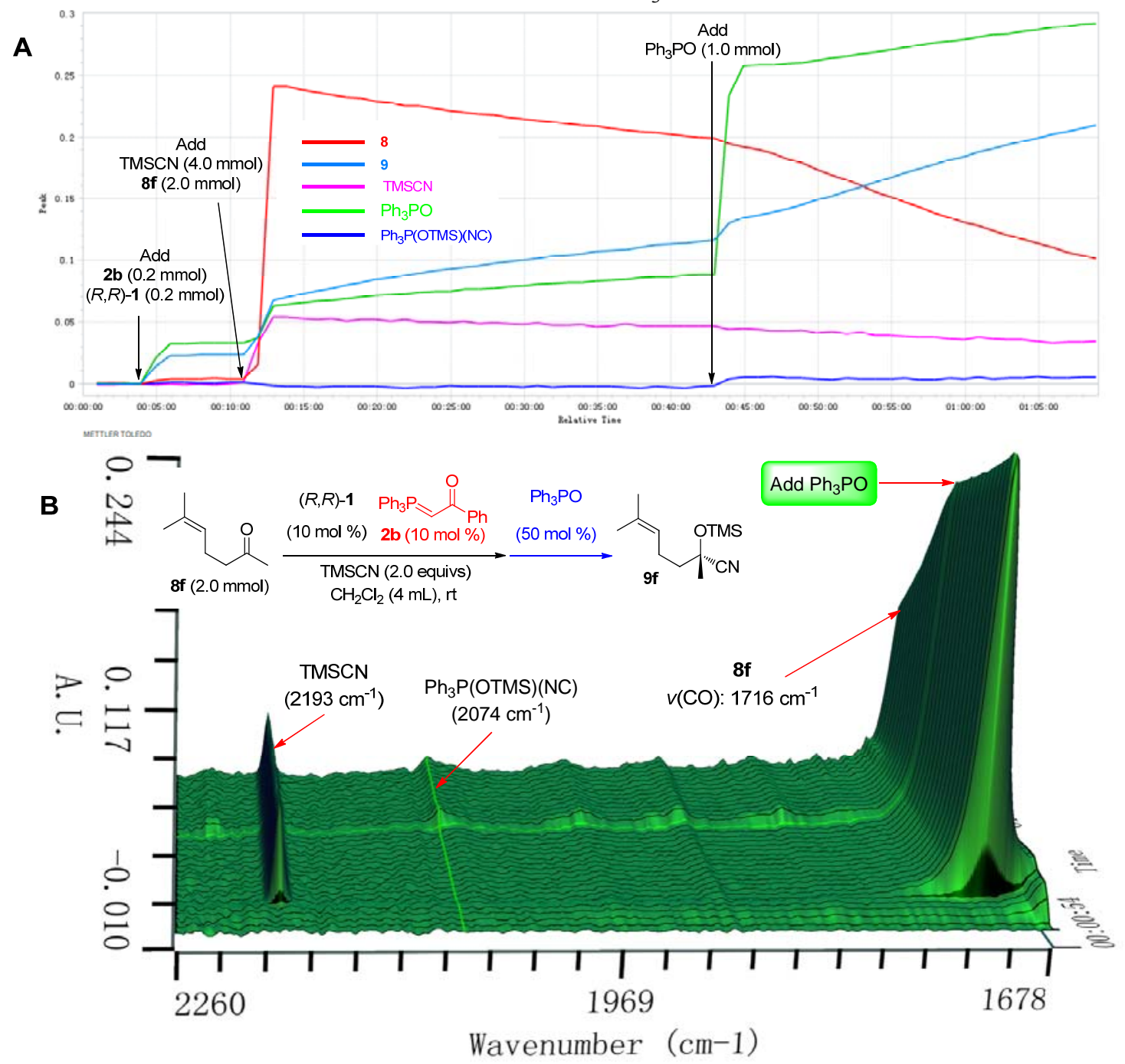




\section{2-5. DFT calculation results and discussion}

According to Gutmann's rules, ${ }^{16}$ upon the coordination of a Lewis base to a Group 13 Lewis acid $\mathrm{AX}_{3}$ (X: halogen atom), the redistribution of electron density would lead to the polarization of the $\mathrm{A}-\mathrm{X}$ bond, which might result in the ionization of $\mathrm{X}$ and formation of a cationic complex with enhanced Lewis acidity. This offers a reasonable explanation for our proposal that the coordination of phosphorane 2a to chiral aluminum complex $(R, R)-\mathbf{1}$, contributes to the formation of cationic chiral complex with enhanced activity. Therefore, we further conducted DFT calculations to investigate in details the binding of phosphorane 2a to chiral (salen)AlCl complex $(R, R)-\mathbf{1}$, through $O$-coordination, results in an activated chiral aluminum catalyst.

Figure S25. Catalyst complexes, which were optimized at the B3LYP/6-31G(d)\&LANL2DZ level. The bond distances of the optimized structures were in angstroms and the NBO charges were in parentheses. These results in solvent and gas phase were denoted in bold and underlined, respectively.
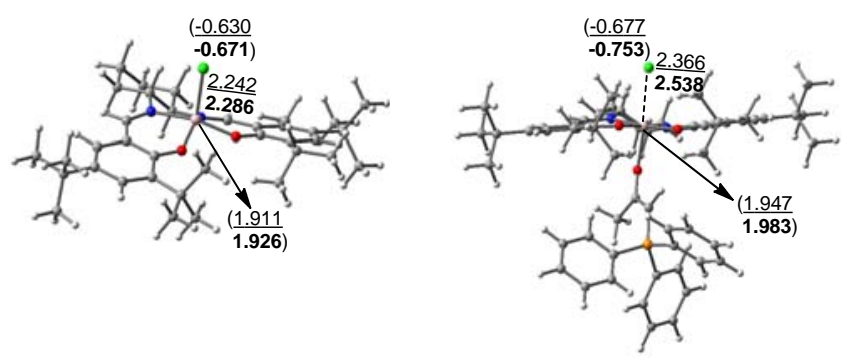

$(R, R)-1$

$(R, R)-\mathbf{1} / \mathbf{2 a}$

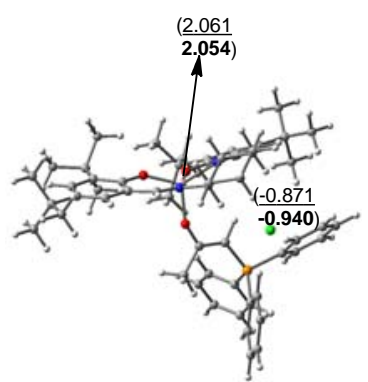

$(R, R)-1 / 2 \mathrm{a} / \mathrm{Cl}-2$

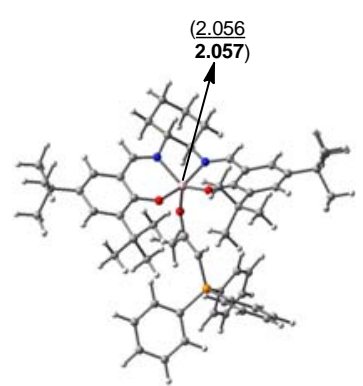

$[(R, R)-\mathbf{1} / \mathbf{2 a}-\mathrm{Cl}]^{+}$

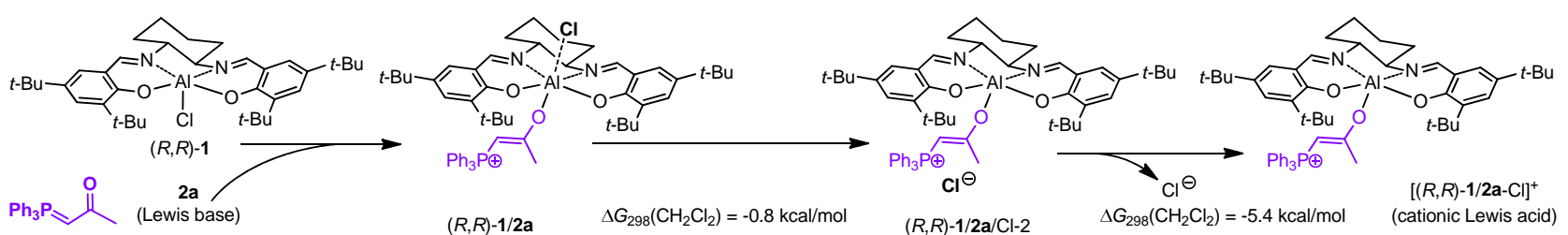

Our theoretical studies showed that the interaction of phosphorane 2a to complex $(R, R)-\mathbf{1}$ indeed polarized the $\mathrm{Al}-\mathrm{Cl}$ bond to form a cationic complex $[(R, R)-\mathbf{1} / \mathbf{2 a}-\mathrm{Cl}]^{+}$. As shown in Figure $\mathrm{S} 25$, when 2a was added to complex $(R, R)-\mathbf{1}$, a complex $(R, R)-\mathbf{1} / \mathbf{2 a}$ formed, and the $\mathrm{Al}-\mathrm{Cl}$ distance in the resulting complex $(R, R)-\mathbf{1} / \mathbf{2 a}$ was obviously longer than that in $(R, R)-\mathbf{1}\left(2.538\right.$ vs $2.286 \AA$ in $\mathrm{CH}_{2} \mathrm{Cl}_{2}$ solvent, 2.366 vs $2.242 \AA$ in gas phase). The NBO analysis also showed that the NBO charge of Al atom in $(R, R)-\mathbf{1} / \mathbf{2 a}$ was more positive than that in $(R, R)-\mathbf{1}\left(1.983\right.$ vs 1.926 in solvent $\mathrm{CH}_{2} \mathrm{Cl}_{2}, 1.947$ vs 1.911 in gas phase), while the chlorine atom has more negative charge (-0.753 vs -0.671 in $\mathrm{CH}_{2} \mathrm{Cl}_{2}$ solvent, -0.677 vs -0.630 in gas phase). These results were consistent with the Gutmann's analysis ${ }^{16 a}$. 
In $\mathrm{CH}_{2} \mathrm{Cl}_{2}$ solvent, the complex $(R, R)-\mathbf{1} / \mathbf{2} \mathbf{a}$ could convert to complex $(R, R)-\mathbf{1} / \mathbf{2} \mathbf{a} / \mathrm{Cl}-2$ with a decrement of Gibbs free energy by $0.8 \mathrm{kcal} / \mathrm{mol}$. In complex $(R, R)-\mathbf{1} / \mathbf{2 a} / \mathrm{Cl}-2$, there was weak interaction between chloride anion and (salen)AlCl complex, which was capable of releasing the weakly bound chloride anion to produce a cationic complex $[(R, R)-\mathbf{1} / \mathbf{2} \mathbf{a}-\mathrm{Cl}]^{+}$with a decrement of Gibbs free energy by $5.4 \mathrm{kcal} / \mathrm{mol}$. These results indicated that the $\mathrm{Al}-\mathrm{Cl}$ bond in $(R, R)-1$ could be readily polarized upon the coordination of phosphorane $\mathbf{2 a}$, leading to the easy releasing of a chloride ion to form complex $(R, R)-\mathbf{1} / \mathbf{2} \mathbf{a} / \mathrm{Cl}-2$, which then produced the cationic complex $[(R, R)-\mathbf{1} / \mathbf{2} \mathbf{a}-\mathrm{Cl}]^{+}$. Considering the cyanosilylation reaction proceeded in solvent, we believed that the adduct $(R, R)-\mathbf{1} / \mathbf{2} \mathbf{a} / \mathrm{Cl}-2$ might release a chlorine anion to produce the cationic complex $[(R, R)-\mathbf{1} / \mathbf{2} \mathbf{a}-\mathrm{Cl}]^{+}$as the real reactive chiral catalytic species, with the chloride ion being protected in the clusters of solvent molecules or other species of the reaction system. Accordingly, we used $[(R, R)-\mathbf{1} / \mathbf{2} \mathbf{a}-\mathrm{Cl}]^{+}$as the catalyst model for further studies.

Figure S26. Two complexes of $(R, R)-\mathbf{1} / \mathbf{6 a}$ and $[\mathbf{2 a} /(R, R)-\mathbf{1} / \mathbf{6} \mathbf{a}-\mathrm{Cl}]^{+}$, which were optimized at the B3LYP/6-31G(d)\&LANL2DZ level. The bond distances of the optimized structures were in angstroms and the NBO charges were in parentheses.

\section{Model I}
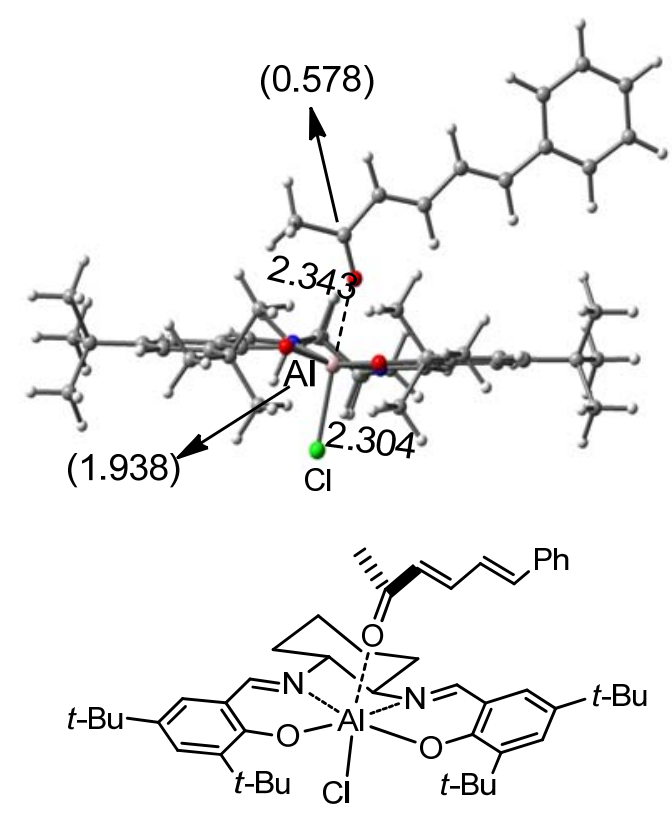

$(R, R)-1 / 6 a$

\section{Model II}

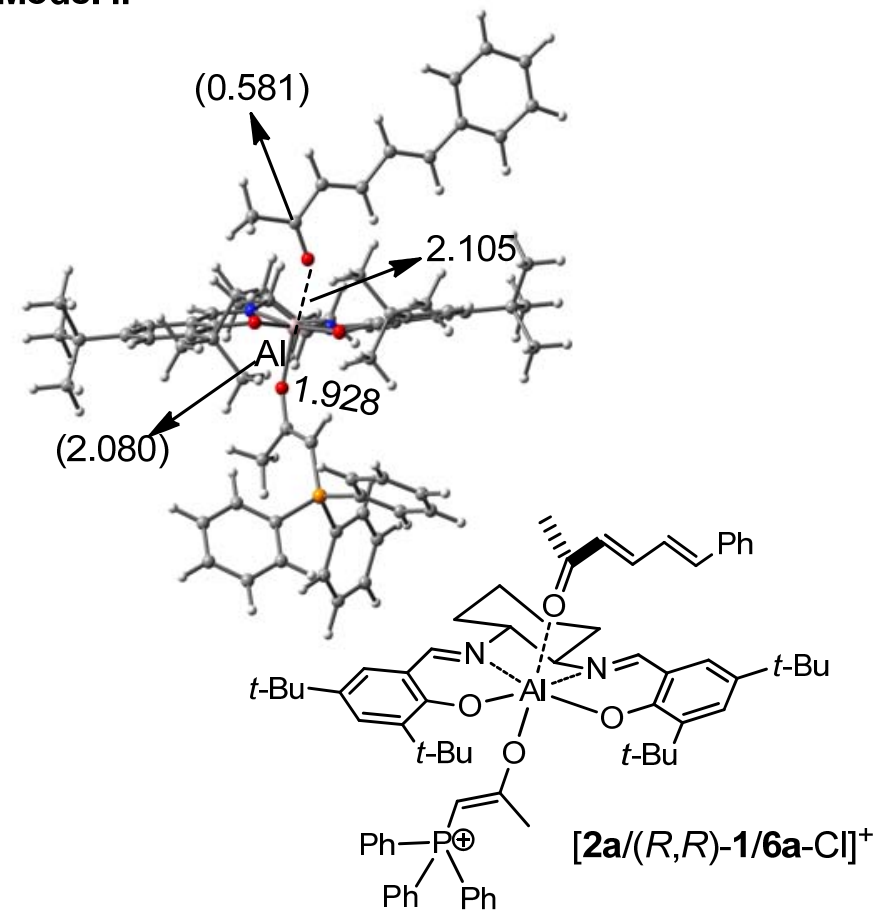

To compare the capacity of complex $(R, R)-\mathbf{1}$ and activated chiral complex $[(R, R)-\mathbf{1} / \mathbf{2} \mathbf{a}-\mathrm{Cl}]^{+}$in activating the electrophilic enone 6a, we further calculated interactions between $(R, R)-\mathbf{1}$ and enone $\mathbf{6 a}$, 
and that between $[(R, R)-\mathbf{1} / \mathbf{2} \mathbf{a}-\mathrm{Cl}]^{+}$and enone $\mathbf{6 a}$, respectively. The calculated results shown in Figure S26 revealed that the activation of enone 6a by complex $(R, R)-\mathbf{1}$ (model I) was less efficient than by cationic complex $[(R, R)-\mathbf{1} / \mathbf{2 a}-\mathrm{Cl}]^{+}$(model $\mathbf{I I}$ ), as the $\mathrm{Al}-\mathrm{O}$ distance in model $\mathbf{I}$ was obviously longer than that in model II (2.343 vs $2.105 \AA$ ). More importantly, the NBO analysis shown that the NBO charge of $\mathrm{Al}$ atom and carbonyl carbon in model II were more positive than those in model I (Al, 2.076 vs 1.938; C, 0.581 vs 0.578$)$, which indicated that cationic complex $[(R, R)-\mathbf{1} / \mathbf{2 a}-\mathrm{Cl}]^{+}$had stronger Lewis acidity to enhance the electrophilicity of the carbonyl group of enone 6a. These results supported that $[(R, R)-\mathbf{1} / \mathbf{2} \mathbf{a}-\mathrm{Cl}]^{+}$activated enone 6a more efficiently, which was in agreement with the fact that without the assistance of $\mathrm{Ph}_{3} \mathrm{PO}$ in activating TMSCN, complex $(R, R)-1$ itself failed to mediate the cyanosilylation of enone $\mathbf{6 a}$ at room temperature, while the merge of $(R, R)-\mathbf{1}$ and $\mathbf{2 a}$ could (entry 1 vs entry 7 of Table S1).

Figure S27. Gibbs free energy profile of the C-C formation step under catalyst free condition

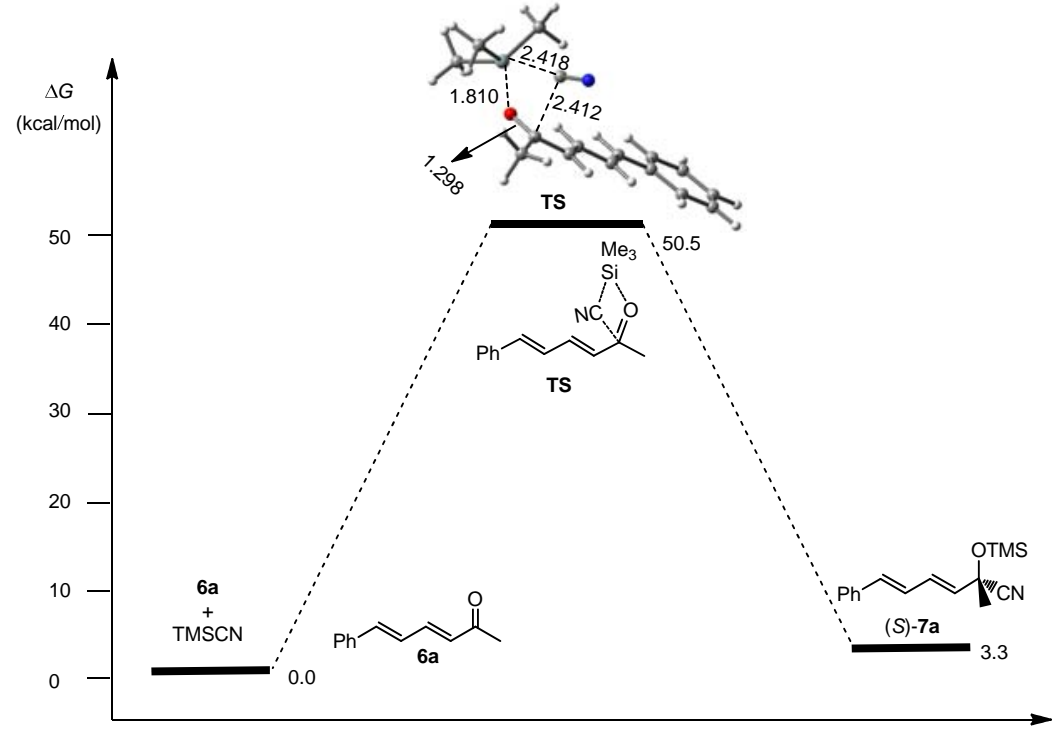

To get more information about the reaction, we further conducted theoretical calculations to study the reaction mechanism. First, we considered the reaction pathway without the use of any catalyst. In previous related theoretical research work ${ }^{17,18}$ on the cyanosilylation mechanism of ketones without catalysts, the DFT calculated results showed that $\mathrm{C}-\mathrm{C}$ addition was the rate-determining step of the reaction and the corresponding activation barriers were very high. Our calculation results were consistent with literature report ${ }^{17}$, as shown in Figure S27. The computed total Gibbs free energy barrier for the reaction without catalyst was $50.5 \mathrm{kcal} / \mathrm{mol}$. Because the activation barrier was so high, the cyanosilylation of enone $6 \mathbf{a}$ could hardly happen without the use of catalyst. 
For the reaction involving the use of chiral aluminum complex, we focused on the theoretical study of the $\mathrm{C}-\mathrm{C}$ bond forming step, the rate-limiting step of the whole reaction. The cyanosilylation of enone 6a mediated by chiral complex $(R, R)-\mathbf{1}$ and the activated chiral aluminum catalyst $[(R, R)-\mathbf{1} / \mathbf{2} \mathbf{a}-\mathrm{Cl}]^{+}$resulting from the binding of phosphorane $\mathbf{2 a}$ to $(R, R)-\mathbf{1}$ was investigated, respectively. The Gibbs free energy profiles of the $\mathrm{C}-\mathrm{C}$ addition step were shown in Figure $\mathrm{S} 28$, and the corresponding optimized structures of the reaction species were present in Figure S29 and S30.

Figure S28. Gibbs free energy profile of the $\mathrm{C}-\mathrm{C}$ formation step of cyanosilylation catalyzed by $(R, R)-\mathbf{1}$ and $[(R, R)-\mathbf{1} / \mathbf{2} \mathbf{a}-\mathrm{Cl}]^{+}$.

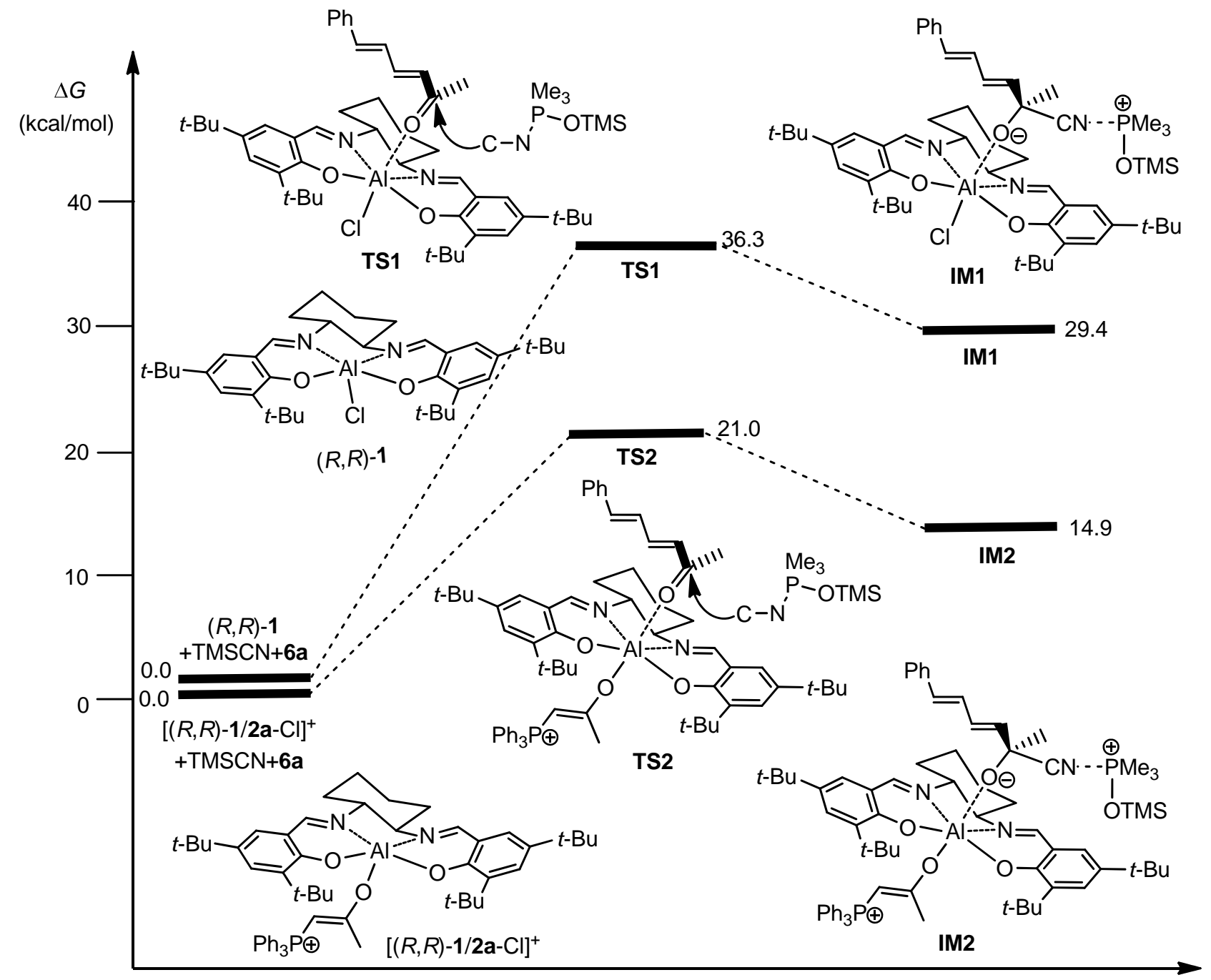

As the previous control experiments and React IR studies had confirmed the indispensable role of $\mathrm{Ph}_{3} \mathrm{PO}$ in achieving desired reactivity, possibly via the activation of TMSCN to form a more reactive nucleophilic isocynide species, $\mathrm{Ph}_{3} \mathrm{P}(\mathrm{OTMS})(\mathrm{N}=\mathrm{C}),{ }^{12,19}$ our present calculation also took into consideration the cooperative activation of TMSCN by tertiary phosphine oxide. But to simplify the 
calculation, we used a much simpler $\mathrm{Me}_{3} \mathrm{PO}$ to replace $\mathrm{Ph}_{3} \mathrm{PO}$ for the calculation model of the reactive isocynide species, ${ }^{20}$ namely $\mathrm{Me}_{3} \mathrm{P}(\mathrm{OTMS})(\mathrm{N}=\mathrm{C})$, to react with activated electrophilic enone $\mathbf{6} \mathbf{a}$.

As shown in Figure S28, the total Gibbs free energy barrier of $\mathrm{C}-\mathrm{C}$ addition step with complex $(R, R)-1$ was $36.3 \mathrm{kcal} / \mathrm{mol}$. Distinct from the $50.5 \mathrm{kcal} / \mathrm{mol}$ barrier of catalyst-free version, the energy barrier could be lowered by $14.2 \mathrm{kcal} / \mathrm{mol}$ when using complex $(R, R)-\mathbf{1}$ to activate enone $\mathbf{6 a}$ and $\mathrm{Me}_{3} \mathrm{PO}$ to activate TMSCN. However, it was still high, which was in accordance with experimental result that the reaction could hardly take place at $-30{ }^{\circ} \mathrm{C}$ (entry 4 of Table S1). In sharp contrast, when using Lewis adduct $[(R, R)-\mathbf{1} / \mathbf{2} \mathbf{a}-\mathrm{Cl}]^{+}$to activate enone $\mathbf{6 a}$, the total Gibbs free energy barrier was further lowered down to $21.0 \mathrm{kcal} / \mathrm{mol}$, which was $15.3 \mathrm{kcal} / \mathrm{mol}$ lower than that of the reaction catalyzed by $(R, R)-\mathbf{1}$. As shown in Figure $\mathrm{S} 30$, the NBO charge of $\mathrm{Al}$ atom and carbonyl carbon in TS2 were more positive than those in TS1 (A1, 2.080 vs 1.952; C, 0.487 vs 0.464), which indicated that the binding of phosphorane 2a to complex $(R, R)-\mathbf{1}$ resulted in stronger Lewis acidity of aluminum center and enhanced electrophilicity of the carbonyl carbon in transition state TS2. The stronger Lewis acidity of $[(R, R)-\mathbf{1} / \mathbf{2} \mathbf{a}-\mathrm{Cl}]^{+}$can disperse more negative charge of the TS2 and then stabilize the transition state. The enhanced electrophilicity of the carbonyl carbon also greatly affected the reaction energy barrier. As a result, the activation of complex $(R, R)-\mathbf{1}$ by phosphorane $\mathbf{2 a}$ effectively decreased the reaction barrier. The present theoretical results provide a rational explanation for our observation that the reaction mediated by a two-catalyst system (10 mol \% $(R, R)-1$ and $\left.100 \mathrm{~mol}_{\%} \mathrm{Ph}_{3} \mathrm{PO}\right)$ afforded product $7 \mathbf{a}$ in less than $5 \%$ yield even after $36 \mathrm{~h}$ at $-30{ }^{\circ} \mathrm{C}$ (entry 4 , Table S1), but that mediated by a three-component catalyst system composed of $10 \mathrm{~mol} \%(R, R)-1,10 \mathrm{~mol} \%$ phosphorane $2 \mathrm{a}$ and $100 \mathrm{~mol} \% \mathrm{Ph}_{3} \mathrm{PO}$ was dramatically accelerated to give $7 \mathbf{a}$ in $88 \%$ yield and $92 \%$ ee (entry 5, Table S1). 
Figure S29. Optimized structures of the species in reaction pathways at the B3LYP/6-31G(d,p)\& LANL2DZ level. The bond distances of the optimized structures were in angstroms.

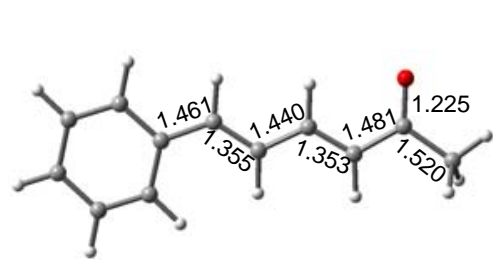<smiles>CC(=O)/C=C/C=C/c1ccccc1</smiles>

$3 a$
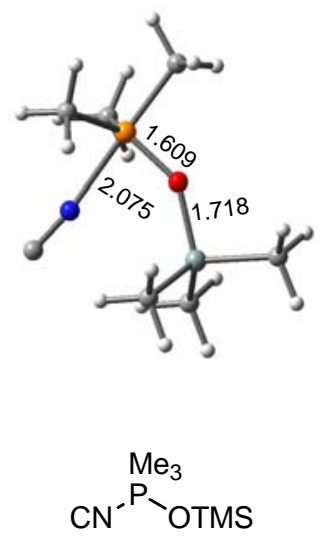

$\mathrm{Me}_{3} \mathrm{P}(\mathrm{OTMS})(\mathrm{N}=\mathrm{C})$
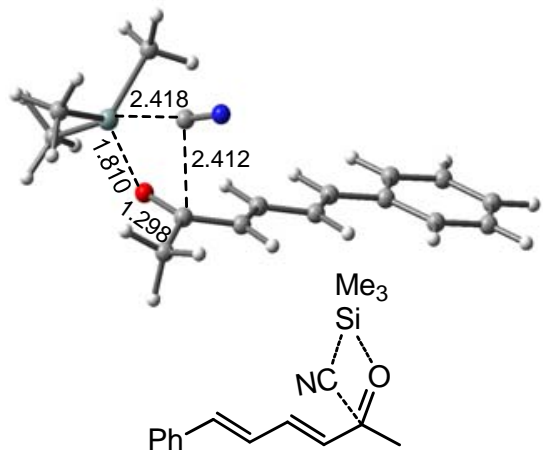

TS
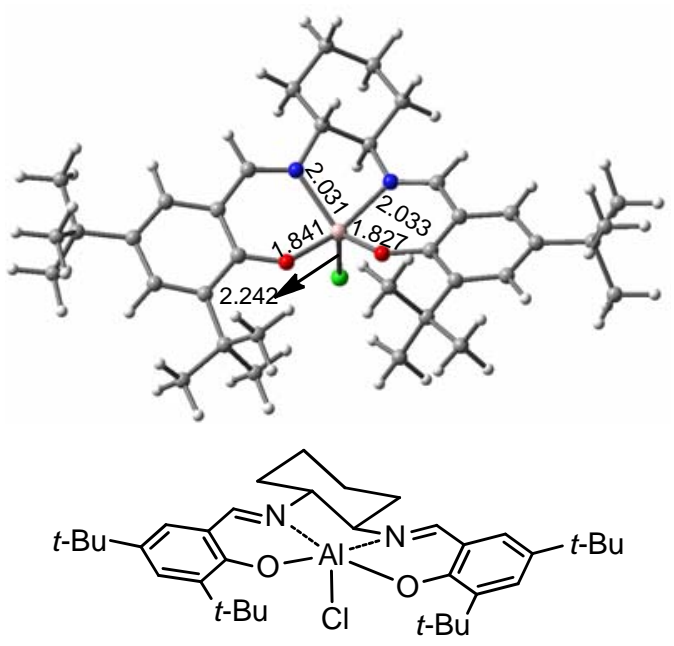

$(R, R)-1$

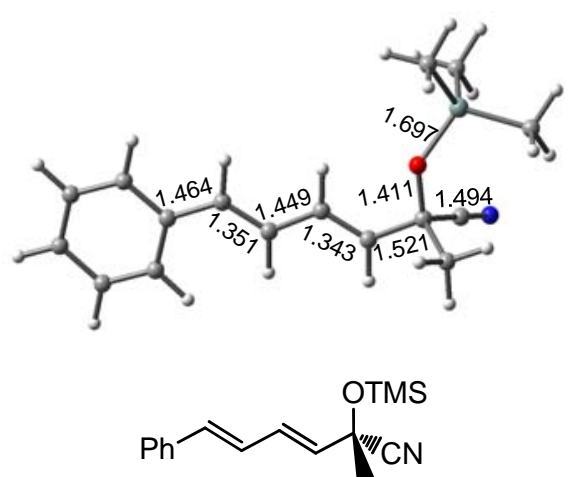

(S)-4a

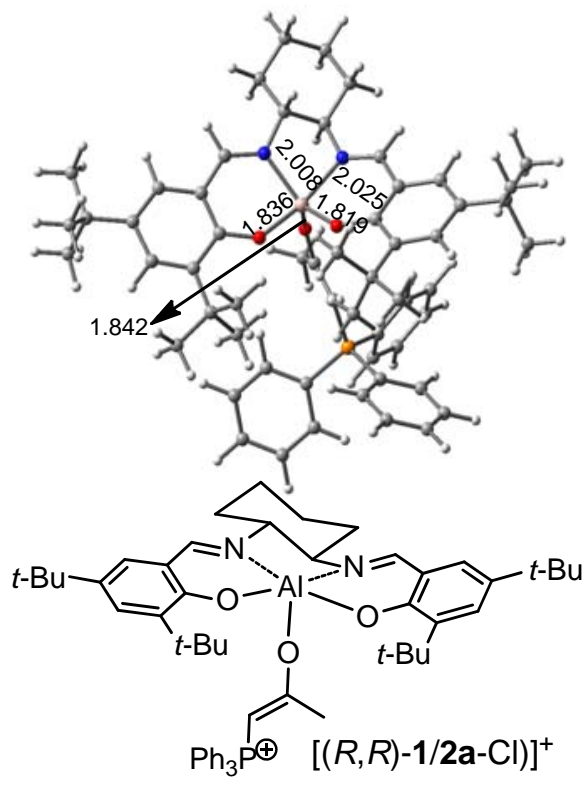


Figure S30. Optimized structures of the TS and intermediates in $\mathrm{C}-\mathrm{C}$ addition step at the B3LYP/6-31G(d,p)\&LANL2DZ level. The bond distances of the optimized structures were in angstroms the NBO charges were in parentheses. The hydrogen atoms were not shown for clarity.
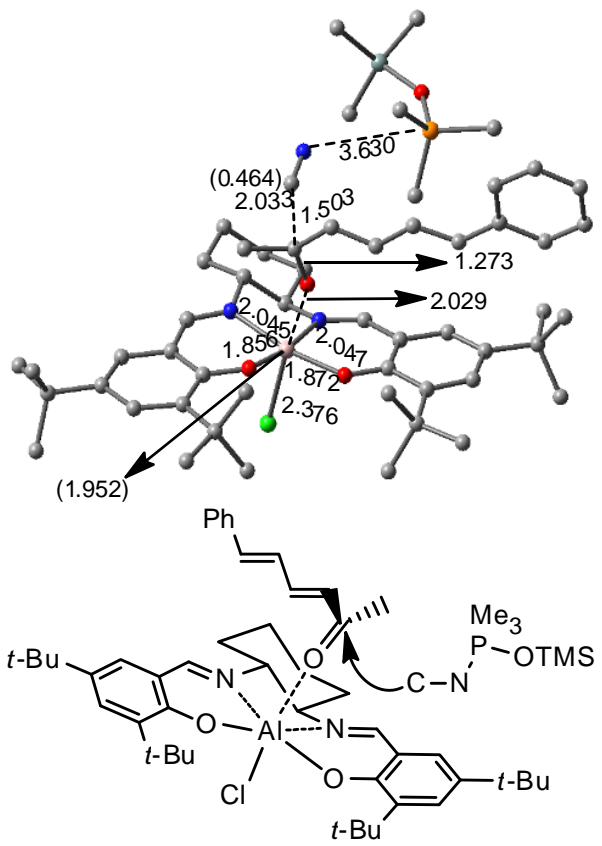

TS1
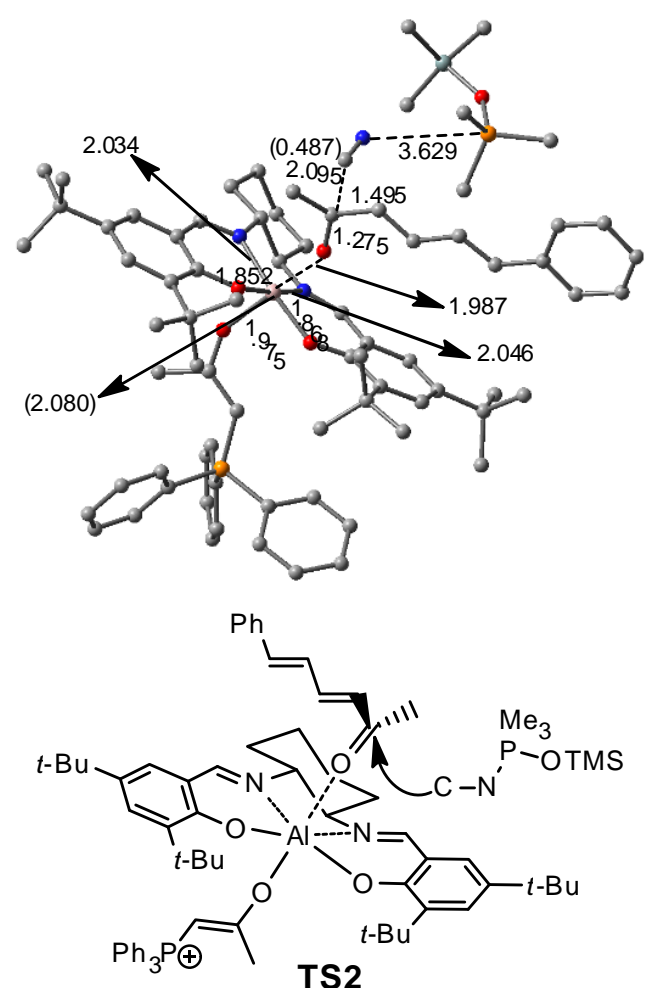
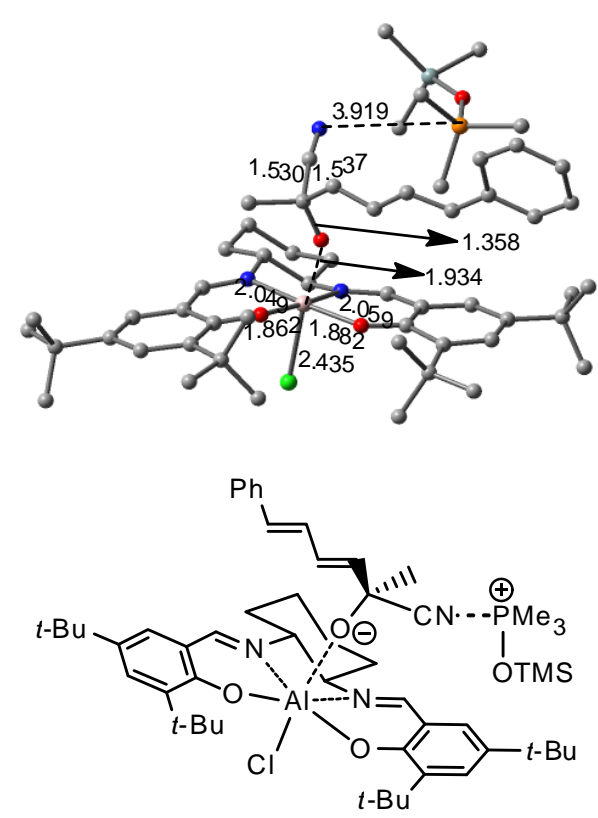

IM1
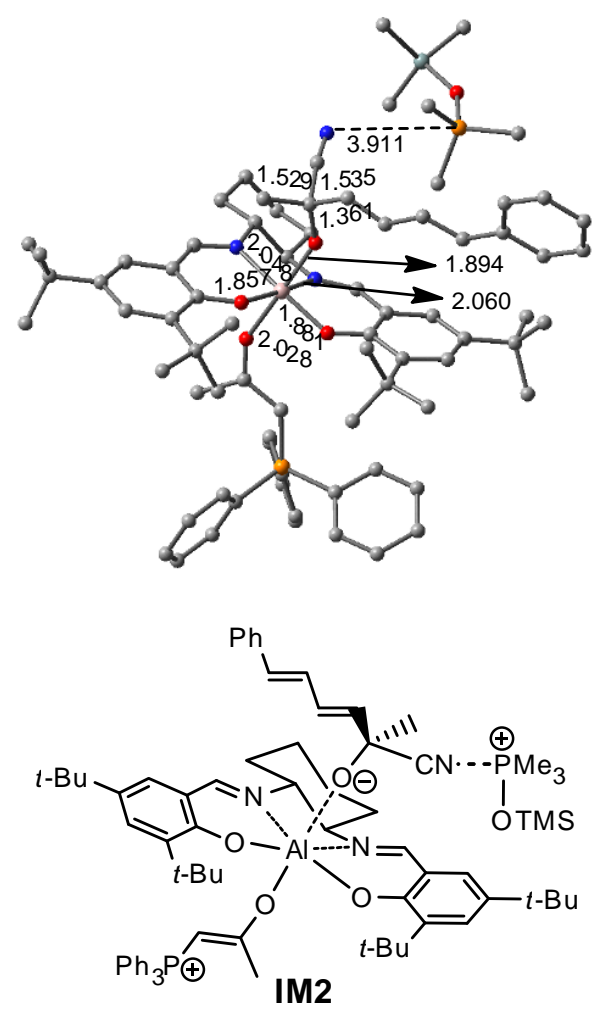


\section{2-6. Electrical conductivity experiments}

General information for the electrical conductivity experiments: All the electrical conductivity experiments were conducted using the SevenCompact ${ }^{\text {TM }}$ S230 conductivity meter from METTLER TOLEDO and its InLab ${ }^{\circledR} 741$-ISM, 2-electrode steel conductivity sensor in anhydrous $\mathrm{CH}_{2} \mathrm{Cl}_{2}$ at 25 ${ }^{\circ} \mathrm{C}$. A general procedure was as follows: to an amber bottle was added anhydrous $\mathrm{CH}_{2} \mathrm{Cl}_{2}(14 \mathrm{~mL})$. After testing the background electrical conductivity, the corresponding solid compound was added in small portions. Each data was collected for three times after the system reached a balance, the average of which was used for the graphic drawing.

First, the conductivity of complex $(R, R)-\mathbf{1}$ or phosphorane 2a in $\mathrm{CH}_{2} \mathrm{Cl}_{2}$ was evaluated, and both proved to be weak: the conductivity of $(R, R)-1$ was $1.52 \mu \mathrm{S} / \mathrm{cm}\left(0.02 \mathrm{~mol} / \mathrm{L}, 25{ }^{\circ} \mathrm{C}\right)$, and that of phosphorane $2 \mathrm{a}$ was $0.68 \mu \mathrm{S} / \mathrm{cm}\left(0.02 \mathrm{~mol} / \mathrm{L}, 25^{\circ} \mathrm{C}\right)$. Then we tested the influence of phosphorane $2 \mathrm{a}$ on the electrical conductivity of a $(R, R)-\mathbf{1}$ in $\mathrm{CH}_{2} \mathrm{Cl}_{2}$ solution. As is illustrated in Figure $\mathrm{S} 31$, a 0.02 $\mathrm{mol} / \mathrm{L}$ solution of (salen) $\mathrm{AlCl}$ complex showed a poor conductivity of $1.52 \mu \mathrm{S} / \mathrm{cm}(\mathrm{X}=0$, green line); however, the portion-wise addition of solid phosphorane 2a dramatically increased the conductivity of the resulting solution. When one equivalent of phosphorane $\mathbf{2 a}(\mathrm{X}=1)$ was added, the conductivity of the solution increased to $598 \mu \mathrm{S} / \mathrm{cm}$. Further increasing the amount of 2a could enhance the conductivity (702 and $752 \mu \mathrm{S} / \mathrm{cm}$, correlating to the $2: 1$ and 3:1 ratio of $\mathbf{2 a : 1}$, respectively).

Figure S31. Influence of phosphorane 2a on the electrical conductivity of a solution of $(R, R)-\mathbf{1}$.

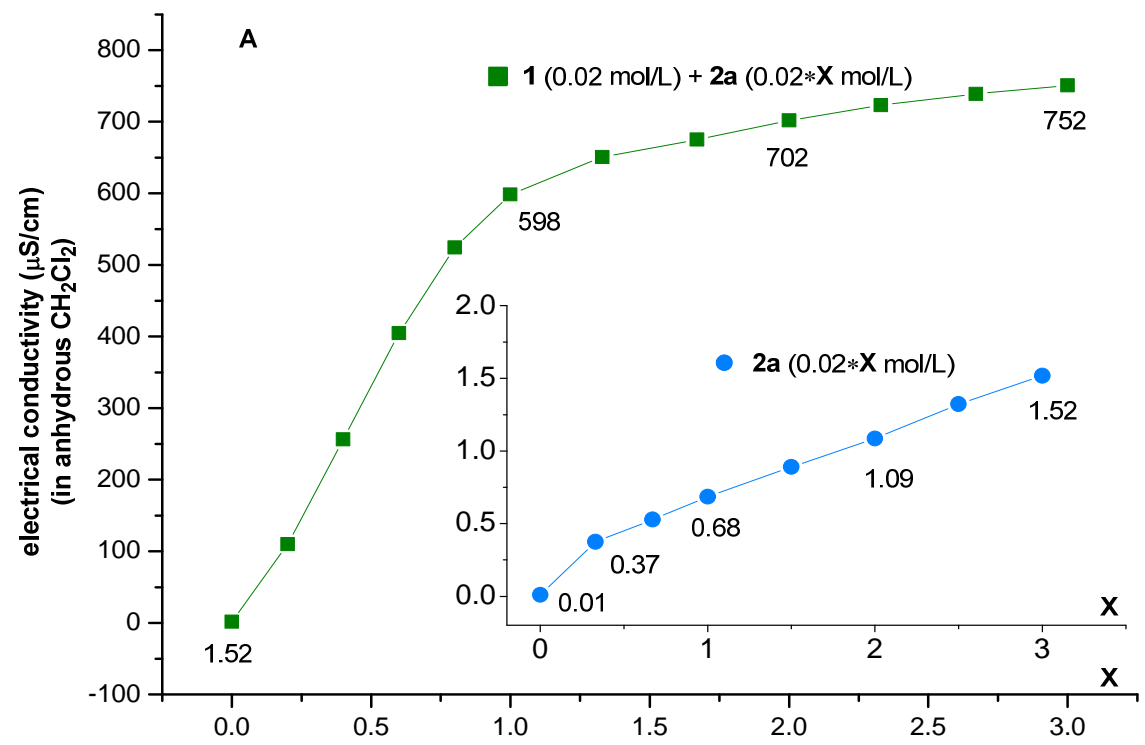

The dramatic change in conductivity was not due to the increase in the concentration of $2 \mathbf{a}$ in the resulting solution, as the observed electrical conductivity for $\mathbf{2 a}$ in $\mathrm{CH}_{2} \mathrm{Cl}_{2}$ with a concentration of 
$0.06 \mathrm{~mol} / \mathrm{L}$ was only $1.52 \mu \mathrm{S} / \mathrm{cm}(\mathrm{X}=3$, blue line, Figure S31). Based on these results, we could safely conclude that the significant growth in the electrical conductivity of the resulting solution originated from the formation of ionic species via the Lewis acid-base interaction between $\mathbf{2 a}$ and $\mathbf{1}$.

Such an enhanced electrical conductivity was also observed when adding $\mathrm{Ph}_{3} \mathrm{PO}$ to the $\mathrm{CH}_{2} \mathrm{Cl}_{2}$ solution of $\mathbf{1}$, as shown in Figure S32. The addition of 1.0 equivalent of $\mathrm{Ph}_{3} \mathrm{PO}$ into a $0.02 \mathrm{~mol} / \mathrm{L}$ solution of (salen) $\mathrm{AlCl}$ complex could afford an electrical conductivity of $149 \mu \mathrm{S} / \mathrm{cm}$ (purple line), almost 10 times of that of the pure (salen) $\mathrm{AlCl}$ complex solution $(1.52 \mu \mathrm{S} / \mathrm{cm})$. Further increase in electrical conductivity to $418,574,650$ and $697 \mu \mathrm{S} / \mathrm{cm}$ was observed with the ratio of $\mathrm{Ph}_{3} \mathrm{PO}:(R, R)-1$ increasing from $3: 1$ to $5: 1,7: 1$ and 10:1, respectively. On the other hand, the pure solution of $\mathrm{Ph}_{3} \mathrm{PO}$ in $\mathrm{CH}_{2} \mathrm{Cl}_{2}$ also turned out to be with poor conductivity (gray line), indicating that the enhanced electrical conductivity was due to the Lewis acid-base interaction between $\mathrm{Ph}_{3} \mathrm{PO}$ and (salen) $\mathrm{AlCl}$ complex $(R, R)-\mathbf{1}$. These results revealed that the interaction of $\mathrm{Ph}_{3} \mathrm{PO}$ with $(R, R)-\mathbf{1}$ might lead to the generation of charged complexes, but not as efficient as phosphorane $\mathbf{2 a}$.

Figure S32. Influence of $\mathrm{Ph}_{3} \mathrm{PO}$ on the electrical conductivity of a solution of $(R, R)-1$

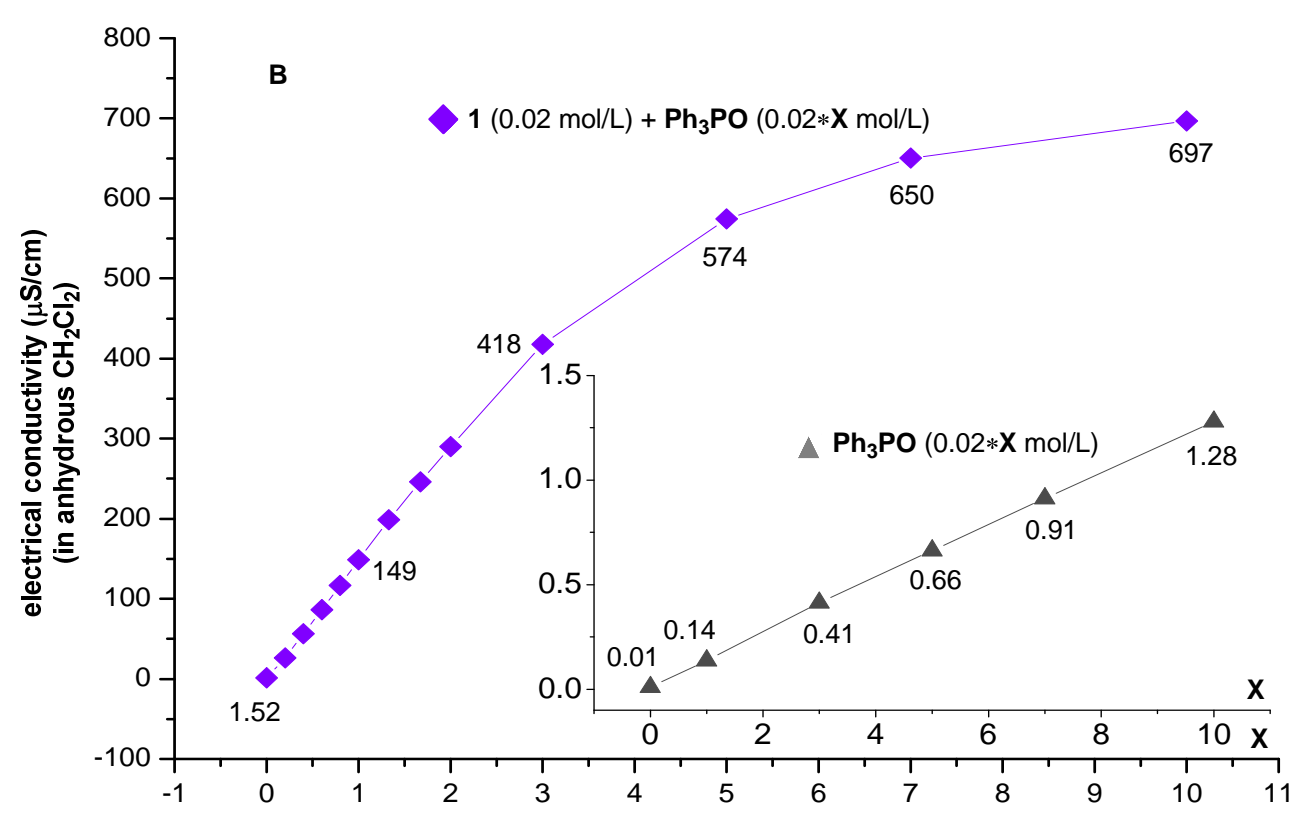

We further determined the change in electrical conductivity of the $\mathrm{CH}_{2} \mathrm{Cl}_{2}$ solution of (salen)AlCl complex $(R, R)-\mathbf{1}\left(0.02 \mathrm{~mol} / \mathrm{L}, 25^{\circ} \mathrm{C}\right)$, after the addition of some typical Lewis bases $\mathbf{L B}_{1-9}$, as some of them had been used to improve reactivity and enantioselectivity of chiral salen complexes mediated reactions. As shown in Figure S33, when any of these Lewis bases, except $\mathrm{Ph}_{3} \mathrm{P}$, was added to a $\mathrm{CH}_{2} \mathrm{Cl}_{2}$ solution of complex $(R, R)-\mathbf{1}$, the conductivity of the resulting solution was dramatically increased, although the conductivity of these substances in $\mathrm{CH}_{2} \mathrm{Cl}_{2}$ at a concentration of $0.02 \mathrm{~mol} / \mathrm{L}$ at 
$25{ }^{\circ} \mathrm{C}$ was generally low, as shown in Table S5. The electrical conductivity of the resulting solution of complex $(R, R)-1$ and a Lewis base in a 1:1 ratio were also summarized in Table S5.

When 1.0 equiv of a given Lewis base was added to the $\mathrm{CH}_{2} \mathrm{Cl}_{2}$ solution of complex $(R, R)-\mathbf{1}$, the highest conductivity was observed when phosphorane 2 a was added (598 $\mu \mathrm{S} / \mathrm{cm}$, entry 2 of Table S5). The use of $N, N$-dimethylpyridin-4-amine $\mathbf{L} \mathbf{B}_{\mathbf{4}}, 1,4$-diazabicyclo[2.2.2] octane $\mathbf{L B}_{3}$ and tertiary amine $N$-oxide $\mathbf{L B}_{2}$ also brought about dramatic increase in conductivity (entries 4-6). Further increasing the amount of Lewis base resulted in continuous change in the conductivity of the resulting solution, but depended on which kind of Lewis base used, as shown in Figure S33.

Figure S33. Influence of $\mathbf{L B}_{1-9}$ on the electrical conductivity of $\mathrm{CH}_{2} \mathrm{Cl}_{2}$ solution of $(R, R)-\mathbf{1}$

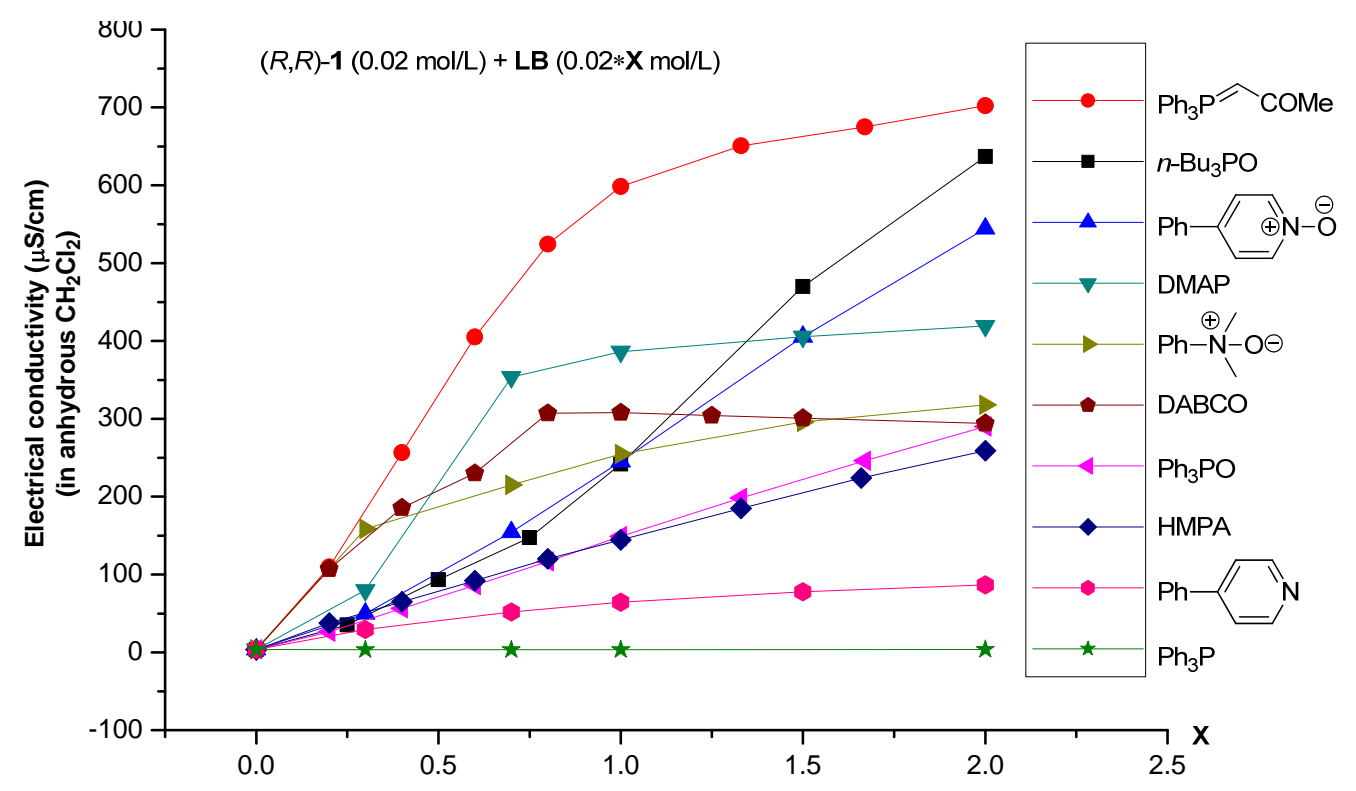

We further examined whether these Lewis bases could facilitate the cyanosilylation of enone $6 \mathbf{a}$, under a condition that the merger of $10 \mathrm{~mol} \%(R, R)-1$ and $100 \mathrm{~mol} \%$ of $\mathrm{Ph}_{3} \mathrm{PO}$ was impotent to promote the reaction (Table S5). As shown in entry 1, almost no reaction took place in the absence of extra Lewis base at $-30{ }^{\circ} \mathrm{C}$ using $\mathrm{CH}_{2} \mathrm{Cl}_{2}$ as the solvent. However, the reaction could proceed with the addition of $10 \mathrm{~mol} \%$ of phosphorane $\mathbf{2} \mathbf{a}, \mathbf{L B}_{2}, \mathbf{L B}_{\mathbf{3}}$ or $\mathbf{L} \mathbf{B}_{\mathbf{4}}$, and phosphorane $\mathbf{2 a}$ exhibited the best acceleration effect, allowing the reaction to finish within $36 \mathrm{~h}$, giving product $7 \mathbf{a}$ in $90 \%$ yield and 92\% ee (entry 2 vs entries 4-6). Almost no reaction took place when other Lewis bases were tried. These results further confirmed that phosphorane 2a had its own advantages over other widely used Lewis basic additives. It should be pointed out that the role of amine $N$-oxide $\mathbf{L B}_{\mathbf{2}}$, tertiary amine $\mathbf{L B}_{\mathbf{3}}$ and DMAP in the reaction was not clear, as they could serve as either as an axial ligand to activate complex $\mathbf{1}$ or as a Lewis base to activate TMSCN. 
Table S5. Compare phosphorane 2a with other activators in the cyanosiliylation of enone 6a
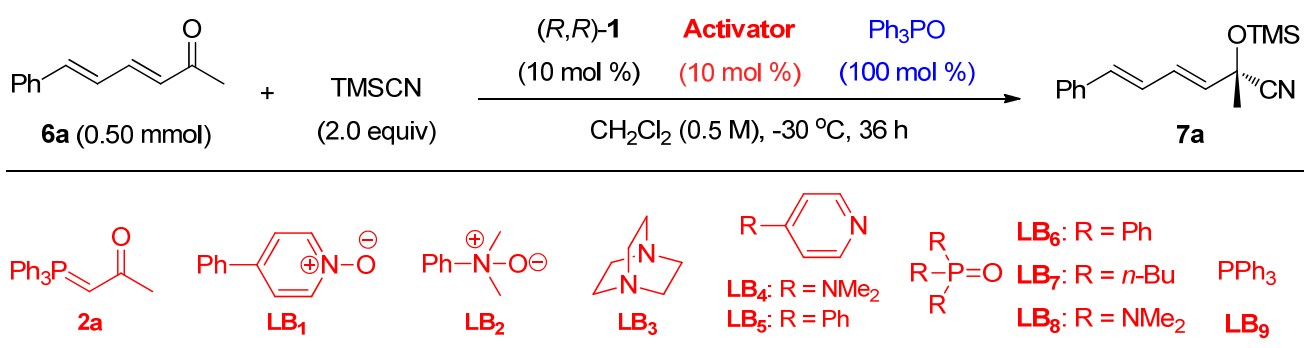

\begin{tabular}{|c|c|c|c|c|c|}
\hline \multirow{2}{*}{ Entry } & \multirow{2}{*}{ Activator } & \multicolumn{2}{|c|}{ Electrical Conductivity $(\mu \mathrm{S} / \mathrm{cm})$} & \multirow{2}{*}{$\begin{array}{l}\text { Yield } \\
(\%)^{\mathrm{a}}\end{array}$} & \multirow{2}{*}{$\begin{array}{c}\mathrm{Ee} \\
(\%)^{\mathrm{b}}\end{array}$} \\
\hline & & Activator $(0.02 \mathrm{M})$ & $\operatorname{Activator} /(R, R)-1(1: 1,0.02 \mathrm{M})$ & & \\
\hline 1 & -- & -- & 1.52 & no reaction & -- \\
\hline 2 & $2 \mathbf{a}$ & 0.685 & 598 & 90 & 92 \\
\hline 3 & $\mathbf{L B}_{1}$ & 0.322 & 245 & trace & -- \\
\hline 4 & $\mathbf{L B}_{2}$ & 1.644 & 255 & 31 & 91 \\
\hline 5 & $\mathbf{L B}_{3}$ & 0.294 & 308 & 65 & 90 \\
\hline 6 & $\mathbf{L B}_{4}$ & 2.84 & 386 & 45 & 89 \\
\hline 7 & $\mathbf{L B}_{5}$ & 0.041 & 64 & trace & -- \\
\hline 8 & $\mathbf{L B}_{6}$ & 0.138 & 149 & trace & -- \\
\hline 9 & $\mathbf{L B}_{7}$ & 0.461 & 242 & trace & -- \\
\hline 10 & $\mathbf{L B}_{8}$ & 0.331 & 144 & trace & -- \\
\hline 11 & $\mathbf{L B}_{9}$ & 0.029 & 3.5 & trace & -- \\
\hline 12 & AgOTf & 0.469 & 37 & no reaction & -- \\
\hline 13 & $\mathrm{AgBF}_{4}$ & 1.860 & 85 & no reaction & -- \\
\hline 14 & $\mathrm{AgSbF}_{6}$ & 68.3 & 188 & no reaction & -- \\
\hline
\end{tabular}

${ }^{\mathrm{a}}$ Isolated yield; ${ }^{\mathrm{b}}$ Determined by HPLC analysis.

We further tried producing cationic aluminum complex by halide abstraction for cyanosilylation reaction. However, after treating complex $(R, R)-1$ with equivalent of $\mathrm{AgOTf} \mathrm{AgBF}_{4}$ or $\mathrm{AgSbF}_{6}$, no reaction took place at all, no matter the resulting $\mathrm{AgCl}$ precipitate was filtered off or not. On the other hand, possibly due to the poor solubility of AgOTf and $\mathrm{AgBF}_{4}$ in $\mathrm{CH}_{2} \mathrm{Cl}_{2}$, their conductivity in $\mathrm{CH}_{2} \mathrm{Cl}_{2}$ at a concentration of $0.02 \mathrm{~mol} / \mathrm{L}$ at $25{ }^{\circ} \mathrm{C}$ was low $(0.469$ and $1.86 \mu \mathrm{S} / \mathrm{cm}$, respectively), and that of $\mathrm{AgSbF}_{6}$ in $\mathrm{CH}_{2} \mathrm{Cl}_{2}$ was much higher because it dissolved well in $\mathrm{CH}_{2} \mathrm{Cl}_{2}$. The conductivity of the resulting $\mathrm{CH}_{2} \mathrm{Cl}_{2}$ solution of $(R, R)-\mathbf{1}$ and any of the three salts in a 1:1 ratio was increased obviously (entries 12-14). Why the resulting (salen)Al(III) complex bearing weakly or non-coordinating counter anion $\mathrm{TfO}^{-}, \mathrm{BF}_{4}^{-}$or $\mathrm{SbF}_{6}^{-}$showed no catalytic activity was unclear at the current stage. These results suggested that the use of a Lewis base to activate a chiral metal complex had its own advantages over the alternative strategy of halide abstraction. 


\section{Asymmetric cyanosilylation of ketone 8}
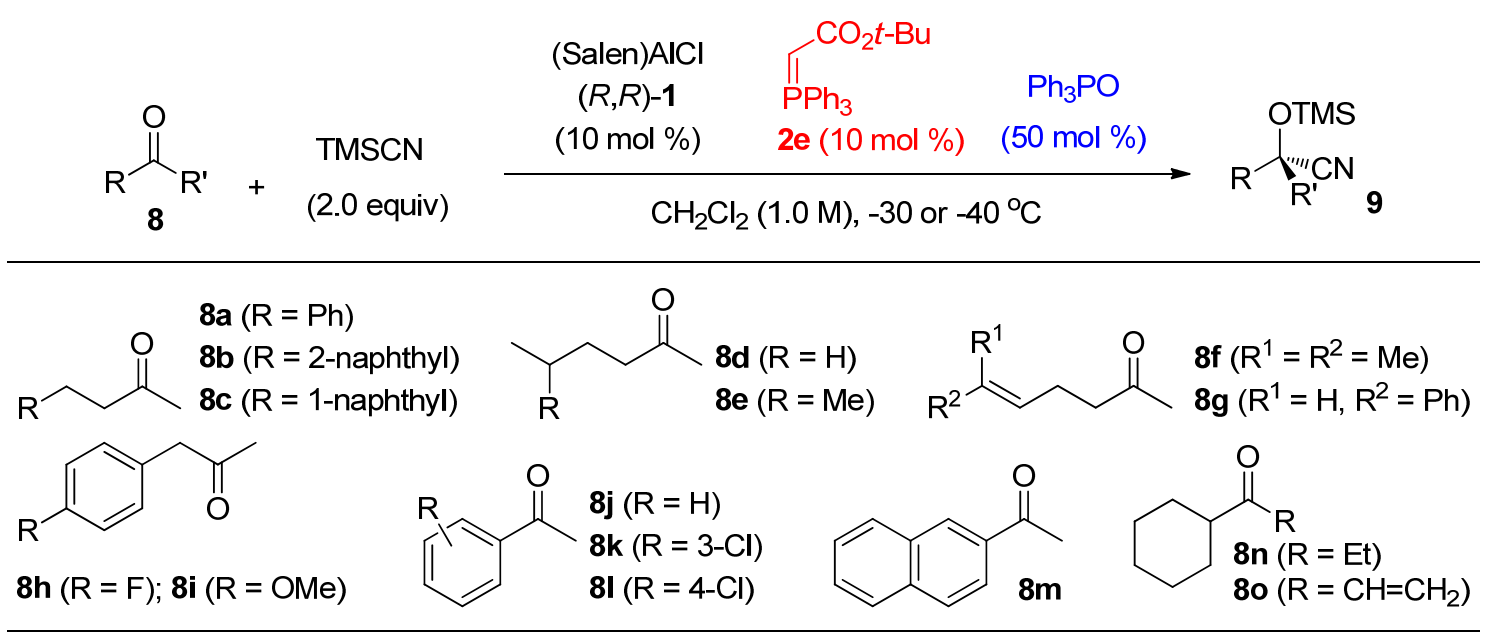

General procedure: To a $4 \mathrm{~mL}$ vial was sequentially added ketone $8(0.5 \mathrm{mmol})$, anhydrous $\mathrm{CH}_{2} \mathrm{Cl}_{2}$ (0.5 mL), (salen) $\mathrm{AlCl}(R, R)-\mathbf{1}(0.05 \mathrm{mmol}, 30.3 \mathrm{mg})$, ylide $2 \mathrm{e}(0.05 \mathrm{mmol}, 17.7 \mathrm{mg})$ and $\mathrm{Ph}_{3} \mathrm{PO}(0.25$ mmol, $69.5 \mathrm{mg}$ ). The resulting solution was stirred at -30 or $-40{ }^{\circ} \mathrm{C}$ for $0.5 \mathrm{~h}$ before TMSCN (1.0 mmol, $125 \mu \mathrm{L}$ ) was charged. After TLC analysis indicated the full consumption of $\mathbf{8}$, the mixture was directly subjected for column chromatograph using petroleum ether/diethyl ether (from 10:0 to 10:1) as the eluent to afford product 9.

OTMS The reaction with 8a was conducted on a $0.5 \mathrm{mmol}$ scale at $-30{ }^{\circ} \mathrm{C}$ for $10 \mathrm{~h}$;
$9{ }^{\prime \prime \prime \prime C N}$ Column chromatography afforded 9a in $97 \%$ yield as colorless oil ${ }^{21}$ HPLC
analysis (Chiralcel OJ-H, ${ }^{i} \mathrm{PrOH} / \mathrm{h}$ exane $=1 / 99,1.0 \mathrm{~mL} / \mathrm{min}, 205 \mathrm{~nm}$; $\mathrm{t}_{\mathrm{r}}$ (major) $=$ $7.19 \mathrm{~min}, \mathrm{t}_{\mathrm{r}}($ minor $\left.)=5.89 \mathrm{~min}\right)$ gave the isomeric composition of the product: $94 \%$ ee; $[\alpha]_{\mathrm{D}}{ }^{25}=-13.3$ (c = 1.1, $\left.\mathrm{CHCl}_{3}\right) ;{ }^{1} \mathrm{H}$ NMR (400 MHz, $\left.\mathrm{CDCl}_{3}\right): \delta$ 7.32-7.20 (m, 5H), 2.91-2.74 (m, 2H), 2.05-2.01 (m, $2 \mathrm{H}), 1.63(\mathrm{~s}, 3 \mathrm{H}), 0.27(\mathrm{~s}, 9 \mathrm{H}) ;{ }^{13} \mathrm{C} \mathrm{NMR}\left(100 \mathrm{MHz}, \mathrm{CDCl}_{3}\right): \delta 140.82,128.62,128.45,126.24$, $121.99,69.44,45.32,30.80,29.11,1.39$.

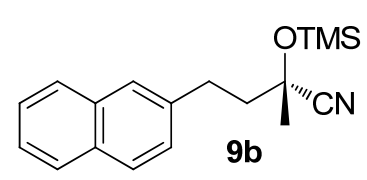

The reaction with $\mathbf{8 b}$ was conducted on a $0.5 \mathrm{mmol}$ scale at $-30{ }^{\circ} \mathrm{C}$ for $10 \mathrm{~h}$; Column chromatography afforded $\mathbf{9 b}$ in $95 \%$ yield as colorless oil; HPLC analysis (Chiralcel OD-H+OD-H, ${ }^{i} \mathrm{PrOH} /$ hexane $=1 / 99,0.5 \mathrm{~mL} / \mathrm{min}, 205$ $\mathrm{nm} ; \mathrm{t}_{\mathrm{r}}($ major $)=35.36 \mathrm{~min}, \mathrm{t}_{\mathrm{r}}($ minor $\left.)=33.59 \mathrm{~min}\right)$ gave the isomeric composition of the product: $92 \%$ ee; $[\alpha]_{\mathrm{D}}{ }^{25}=-10.8\left(\mathrm{c}=1.15, \mathrm{CHCl}_{3}\right) ;{ }^{1} \mathrm{H} \mathrm{NMR}\left(400 \mathrm{MHz}, \mathrm{CDCl}_{3}\right): \delta 7.83-7.78(\mathrm{~m}, 3 \mathrm{H}), 7.66(\mathrm{~s}$, $1 \mathrm{H}), 7.47-7.43(\mathrm{~m}, 2 \mathrm{H}), 7.35(\mathrm{dd}, J=8.4,1.7 \mathrm{~Hz}, 1 \mathrm{H}), 3.06-2.96(\mathrm{~m}, 2 \mathrm{H}), 2.17-2.08(\mathrm{~m}, 2 \mathrm{H}), 1.67$ (s, $3 \mathrm{H}), 0.30(\mathrm{~s}, 9 \mathrm{H}) ;{ }^{13} \mathrm{C} \mathrm{NMR}\left(100 \mathrm{MHz}, \mathrm{CDCl}_{3}\right): \delta 138.30,133.72,132.18,128.23,127.71,127.49$, 127.17, 126.55, 126.15, 125.44, 122.02, 69.51, 45.25, 30.99, 29.16, 1.42.; IR (KBr) v 3052.9, 2958.0, 
2359.9, 1508.9, 1375.9, 1253.9, 1185.6, 1043.8, 819.1, $753.9 \mathrm{~cm}^{-1}$; MS (EI): 297 (M+ , 45), 267 (17), 207 (100), 192 (19), 155 (72), 141 (89), 115 (44); HRMS (EI): Exact mass calcd for $\mathrm{C}_{18} \mathrm{H}_{23} \mathrm{NOSi}$ : 297.1549, found: 297.1546.

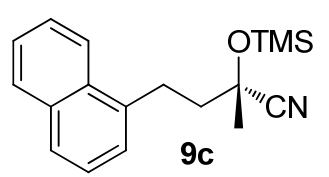

The reaction with $8 \mathbf{c}$ was conducted on a $0.5 \mathrm{mmol}$ scale at $-40{ }^{\circ} \mathrm{C}$ for $15 \mathrm{~h}$; Column chromatography afforded 9c in 97\% yield as colorless oil; HPLC analysis (Chiralcel OD-H+OD-H, ${ }^{i} \mathrm{PrOH} /$ hexane $=1 / 99,1.0 \mathrm{~mL} / \mathrm{min}, 205 \mathrm{~nm}$; $\mathrm{t}_{\mathrm{r}}($ major $)=18.21 \mathrm{~min}, \mathrm{t}_{\mathrm{r}}($ minor $\left.)=19.06 \mathrm{~min}\right)$ gave the isomeric composition of the product: $91 \%$ ee; $[\alpha]_{\mathrm{D}}{ }^{25}=-8.9\left(\mathrm{c}=1.09, \mathrm{CHCl}_{3}\right) ;{ }^{1} \mathrm{H} \mathrm{NMR}\left(400 \mathrm{MHz}, \mathrm{CDCl}_{3}\right): \delta 8.06(\mathrm{~d}, J=8.4 \mathrm{~Hz}, 1 \mathrm{H}), 7.86(\mathrm{~m}, 1 \mathrm{H})$, $7.74(\mathrm{~d}, \mathrm{~J}=8.0 \mathrm{~Hz}, 1 \mathrm{H}), 7.55-7.49(\mathrm{~m}, 2 \mathrm{H}), 7.41-7.37(\mathrm{~m}, 2 \mathrm{H}), 3.38-3.24(\mathrm{~m}, 2 \mathrm{H}), 2.16-2.12(\mathrm{~m}, 2 \mathrm{H})$, $1.69(\mathrm{~s}, 3 \mathrm{H}), 0.33(\mathrm{~s}, 9 \mathrm{H}) ;{ }^{13} \mathrm{C} \mathrm{NMR}\left(100 \mathrm{MHz}, \mathrm{CDCl}_{3}\right): \delta 136.90,134.02,131.74,128.96,127.14$, $126.19,126.18,125.69,125.68,123.50,122.02,69.54,44.56,29.12,28.02,1.42$; IR (KBr) v 2959.0, 2359.7, 1510.6, 1396.1, 1254.2, 1185.0, 1115.1, 1051.6, 798.9, 778.1 cm $\mathrm{cm}^{-1}$ MS (EI): $297\left(\mathrm{M}^{+}, 62\right)$, 267 (10), 207 (71), 192 (46), 155 (58), 141 (100), 115 (50); HRMS (EI): Exact mass calcd for $\mathrm{C}_{18} \mathrm{H}_{23} \mathrm{NOSi}$ : 297.1549, found: 297.1551.

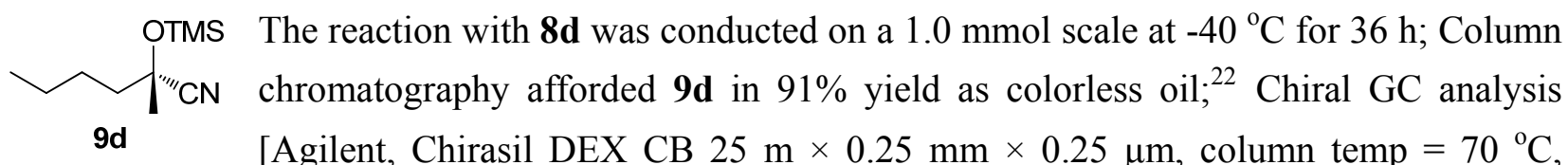
Injector temp $=200{ }^{\circ} \mathrm{C}$, Detector temp $=250{ }^{\circ} \mathrm{C}$, Inlet pressure $\left.=70 \mathrm{kPa}\right), \mathrm{t}_{\mathrm{r}}$ (major) $=25.43 \mathrm{~min}, \mathrm{t}_{\mathrm{r}}$ $($ minor $)=24.88 \mathrm{~min}$. $]$ gave the isomeric composition of the product: $92 \%$ ee; $[\alpha]_{\mathrm{D}}{ }^{25}=-13.9(\mathrm{c}=0.95$, $\left.\mathrm{CHCl}_{3}\right) ;{ }^{1} \mathrm{H}$ NMR $\left(400 \mathrm{MHz}, \mathrm{CDCl}_{3}\right): \delta 1.78-1.65(\mathrm{~m}, 2 \mathrm{H}), 1.56(\mathrm{~s}, 3 \mathrm{H}), 1.54-1.31(\mathrm{~m}, 4 \mathrm{H}), 0.93(\mathrm{t}, J$ $=7.2 \mathrm{~Hz}, 3 \mathrm{H}), 0.23(\mathrm{~s}, 9 \mathrm{H}) ;{ }^{13} \mathrm{C} \mathrm{NMR}\left(100 \mathrm{MHz}, \mathrm{CDCl}_{3}\right): \delta 122.33,69.77,43.22,28.99,26.48,22.53$, $13.99,1.39$.

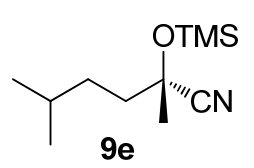

The reaction with $8 \mathrm{e}$ was conducted on a $1.0 \mathrm{mmol}$ scale at $-40{ }^{\circ} \mathrm{C}$ for $36 \mathrm{~h}$; Column chromatography afforded 9e in 97\% yield as colorless oil; Chiral GC analysis [Agilent, Chirasil DEX CB $25 \mathrm{~m} \times 0.25 \mathrm{~mm} \times 0.25 \mu \mathrm{m}$, column temp $=70{ }^{\circ} \mathrm{C}$, Injector temp $=200{ }^{\circ} \mathrm{C}$, Detector temp $=250{ }^{\circ} \mathrm{C}$, Inlet pressure $\left.=70 \mathrm{kPa}\right), \mathrm{t}_{\mathrm{r}}($ major $)=41.45 \mathrm{~min}, \mathrm{t}_{\mathrm{r}}$ $($ minor $)=39.38 \mathrm{~min}$.$] gave the isomeric composition of the product: 92 \%$ ee; $[\alpha]_{\mathrm{D}}{ }^{25}=-12.5(\mathrm{c}=0.95$, $\mathrm{CHCl}_{3}$ ); ${ }^{1} \mathrm{H}$ NMR (400 MHz, $\left.\mathrm{CDCl}_{3}\right): \delta$ 1.78-1.64 (m, 2H), 1.60-1.54 (m, 4H), 1.45-1.31 (m, 2H), $0.91(\mathrm{~d}, J=6.6 \mathrm{~Hz}, 6 \mathrm{H}), 0.24(\mathrm{~s}, 9 \mathrm{H}) ;{ }^{13} \mathrm{C} \mathrm{NMR}\left(100 \mathrm{MHz}, \mathrm{CDCl}_{3}\right): \delta 122.31,69.89,41.45,33.23$, 28.97, 28.00, 22.60, 22.53, 1.40. IR (KBr) v 2958.8, 2872.8, 1469.3, 1376.5, 1254.4, 1191.0, 1147.7, 1102.6, 1079.8, 846.7, 757.0 cm-1; MS (EI): 213 (M+, 5.7), 198.0 (99.9), 171 (99.8), 143 (99.8), 127 (67.7), 102 (73.5), 74 (100); HRMS (EI): Exact mass calcd for C11H23NOSi: 213.1549, found: 213.1546 . 


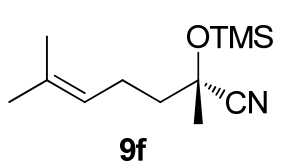

The reaction with $8 \mathbf{f}$ was conducted on a $1.0 \mathrm{mmol}$ scale at $-30{ }^{\circ} \mathrm{C}$ for $20 \mathrm{~h}$; Column chromatography afforded 9 f in $95 \%$ yield as colorless oil; The enantiomeric excess of $9 f$ was determined to be $90 \%$ by HPLC analysis of the corresponding free cyanohydrin [Chiralpak AS-H, ${ }^{i} \mathrm{PrOH} / \mathrm{h}$ exane $=3 / 97,1.0 \mathrm{~mL} / \mathrm{min}, 205 \mathrm{~nm} ; \mathrm{t}_{\mathrm{r}}$ $($ major $)=11.82 \mathrm{~min}, \mathrm{t}_{\mathrm{r}}($ minor $\left.\left.)=10.85 \mathrm{~min}\right)\right] ;[\alpha]_{\mathrm{D}}{ }^{25}=-14.4\left(\mathrm{c}=0.89, \mathrm{CHCl}_{3}\right) ;{ }^{1} \mathrm{H} \mathrm{NMR}(400 \mathrm{MHz}$, $\left.\mathrm{CDCl}_{3}\right): \delta 5.12-5.08(\mathrm{~m}, 1 \mathrm{H}), 2.23-2.13(\mathrm{~m}, 2 \mathrm{H}), 1.79-1.69(\mathrm{~m}, 5 \mathrm{H}), 1.63(\mathrm{~s}, 3 \mathrm{H}), 1.57(\mathrm{~s}, 3 \mathrm{H}), 0.24(\mathrm{~s}$, $9 \mathrm{H}) ;{ }^{13} \mathrm{C}$ NMR $\left(100 \mathrm{MHz}, \mathrm{CDCl}_{3}\right): \delta 132.88,122.59,122.16,69.50,43.39,29.01,25.73,23.13,17.73$, 1.38; IR (KBr) v 2963.8, 2925.5, 2860.0, 1451.0, 1377.8, 1255.0, 1186.0, 1046.3, 843.4, $757.2 \mathrm{~cm}^{-1}$; MS (EI): 225 (M+, 0.25), 207 (6.20), 183 (1.97), 142 (48.4), 135 (61.0), 120 (100), 93 (30.4); HRMS (EI): Exact mass calcd for $\mathrm{C}_{12} \mathrm{H}_{23} \mathrm{NOSi}$ : 225.1549, found: 225.1546 .

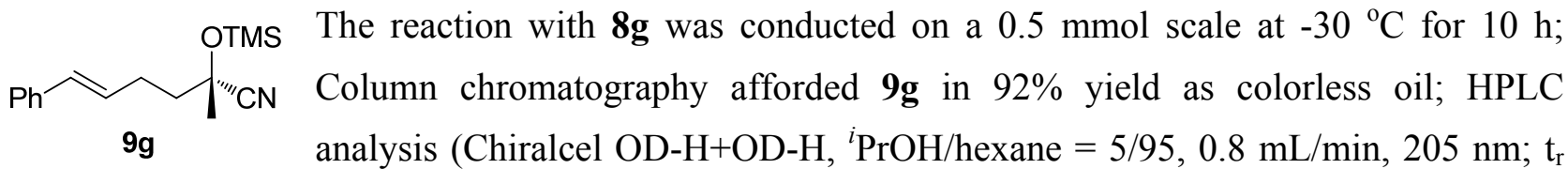
$($ major $)=15.50 \mathrm{~min}, \mathrm{t}_{\mathrm{r}}($ minor $\left.)=14.68 \mathrm{~min}\right)$ gave the isomeric composition of the product: $91 \%$ ee; $[\alpha]_{\mathrm{D}}{ }^{25}=-10.5\left(\mathrm{c}=1.0, \mathrm{CHCl}_{3}\right) ;{ }^{1} \mathrm{H}$ NMR $\left(400 \mathrm{MHz}, \mathrm{CDCl}_{3}\right): \delta 7.35-7.19(\mathrm{~m}, 5 \mathrm{H}), 6.44(\mathrm{~d}, J=15.6$ $\mathrm{Hz}, 1 \mathrm{H}), 6.24-6.17(\mathrm{~m}, 1 \mathrm{H}), 2.52-2.35(\mathrm{~m}, 2 \mathrm{H}), 1.94-1.87(\mathrm{~m}, 2 \mathrm{H}), 1.62(\mathrm{~s}, 3 \mathrm{H}), 0.26(\mathrm{~s}, 9 \mathrm{H}) ;{ }^{13} \mathrm{C}$ NMR $\left(100 \mathrm{MHz}, \mathrm{CDCl}_{3}\right): \delta 137.51,130.88,128.80,128.61,127.19,126.07,122.02,69.37,43.03$, 29.12, 28.01, 1.38; IR (KBr) v 3026.2, 2988.7, 2857.2, 1447.2, 1376.4, 1254.2, 1186.4, 1075.3, 754.0, $693.3 \mathrm{~cm}^{-1}$; MS (EI): $273\left(\mathrm{M}^{+}, 11\right), 258$ (8), 182 (64), 168 (43), 156 (20), 129 (100), 116 (92); HRMS (EI): Exact mass calcd for $\mathrm{C}_{16} \mathrm{H}_{23} \mathrm{NOSi}$ : 273.1549, found: 273.1552 .

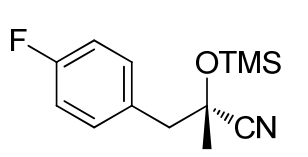

The reaction with $\mathbf{8 h}$ was conducted on a $0.5 \mathrm{mmol}$ scale at $-30{ }^{\circ} \mathrm{C}$ for $6 \mathrm{~h}$; Column chromatography afforded $\mathbf{9 h}$ in $85 \%$ yield as colorless oil; HPLC analysis

(Chiralcel OD-H, ${ }^{i} \mathrm{PrOH} /$ hexane $=0.5 / 95.5,0.4 \mathrm{~mL} / \mathrm{min}, 205 \mathrm{~nm} ; \mathrm{t}_{\mathrm{r}}$ (major) $=$ $14.02 \mathrm{~min}, \mathrm{t}_{\mathrm{r}}($ minor $\left.)=13.43 \mathrm{~min}\right)$ gave the isomeric composition of the product: $92 \%$ ee; $[\alpha]_{\mathrm{D}}{ }^{25}=$ -10.7 (c = 0.92, $\left.\mathrm{CHCl}_{3}\right) ;{ }^{1} \mathrm{H} \mathrm{NMR}\left(400 \mathrm{MHz} \mathrm{CDCl}_{3}\right): \delta$ 7.27-7.23 (m, 2H), 7.04-6.99 (m, 2H), 2.99-2.92 (AB, $J=13.6 \mathrm{~Hz}, 2 \mathrm{H}), 1.58(\mathrm{~s}, 3 \mathrm{H}), 0.14(\mathrm{~s}, 9 \mathrm{H}) ;{ }^{13} \mathrm{C} \mathrm{NMR}\left(100 \mathrm{MHz}, \mathrm{CDCl}_{3}\right): \delta 162.44(\mathrm{~d}$, $J=245.7 \mathrm{~Hz}), 132.28(\mathrm{~d}, J=8.0 \mathrm{~Hz}), 130.55(\mathrm{~d}, J=3.3 \mathrm{~Hz}), 121.57,115.11(\mathrm{~d}, J=21.3 \mathrm{~Hz}), 69.79$ (d, $J=1.4 \mathrm{~Hz}), 48.40,28.94,1.09 ;{ }^{19} \mathrm{~F}$ NMR $\left(376 \mathrm{MHz}, \mathrm{CDCl}_{3}\right): \delta-115.40$; IR $(\mathrm{KBr}) v 2960.3$, 2359.7, 1606.5, 1511.7, 1375.8, 1254.6, 1174.6, 1112.3, 1007.5, 847.2 $\mathrm{cm}^{-1}$; MS (EI): $251\left(\mathrm{M}^{+}, 17\right)$, 236 (5.3), 209 (32), 142 (54), 100 (100), 84 (6.3), 73 (52); HRMS (EI): Exact mass calcd for $\mathrm{C}_{13} \mathrm{H}_{18} \mathrm{NOSiF}: 251.1142$, found: 251.1143 . 
$\mathrm{MeO}$<smiles>CO[C@](C)(C#N)Cc1ccc(C)cc1</smiles>

9i

The reaction with $8 \mathbf{i}$ was conducted on a $0.5 \mathrm{mmol}$ scale at $-40{ }^{\circ} \mathrm{C}$ for $10 \mathrm{~h}$; Column chromatography afforded $\mathbf{9 i}$ in $96 \%$ yield as colorless oil ${ }^{23}$ HPLC analysis $\left(\right.$ Chiralcel OJ-H, ${ }^{i} \mathrm{PrOH} / \mathrm{hexane}=1 / 99,1.0 \mathrm{~mL} / \mathrm{min}, 230 \mathrm{~nm}$; $\mathrm{t}_{\mathrm{r}}$ (major) $=8.48 \mathrm{~min}, \mathrm{t}_{\mathrm{r}}($ minor $\left.)=9.96 \mathrm{~min}\right)$ gave the isomeric composition of the product: $91 \%$ ee; $[\alpha]_{\mathrm{D}}{ }^{25}=-6.7$ $\left(\mathrm{c}=1.05, \mathrm{CHCl}_{3}\right) ;{ }^{1} \mathrm{H} \mathrm{NMR}\left(400 \mathrm{MHz}, \mathrm{CDCl}_{3}\right): \delta 7.20(\mathrm{~d}, \mathrm{~J}=8.7 \mathrm{~Hz}, 2 \mathrm{H}), 6.86(\mathrm{~d}, \mathrm{~J}=8.7 \mathrm{~Hz}, 2 \mathrm{H})$, $3.80(\mathrm{~s}, 3 \mathrm{H}), 2.98-2.89(\mathrm{AB}, \mathrm{J}=13.6 \mathrm{~Hz}, 2 \mathrm{H}), 1.55(\mathrm{~s}, 3 \mathrm{H}), 0.15(\mathrm{~s}, 9 \mathrm{H}) ;{ }^{13} \mathrm{C} \mathrm{NMR}(100 \mathrm{MHz}$, $\left.\mathrm{CDCl}_{3}\right): \delta 159.08,131.76,126.87,121.86,113.67,70.08,55.31,48.43,28.74,1.17$.

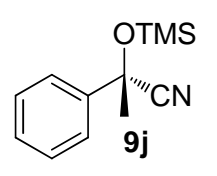

The reaction with $\mathbf{8 j}$ was conducted on a $0.5 \mathrm{mmol}$ scale at $-30{ }^{\circ} \mathrm{C}$ for 3 days; Column chromatography afforded $9 \mathbf{j}$ in $56 \%$ yield as colorless oil ${ }^{24}$ Chiral GC analysis [Agilent, Chirasil DEX CB $25 \mathrm{~m} \times 0.25 \mathrm{~mm} \times 0.25 \mu \mathrm{m}$, column temp $=100{ }^{\circ} \mathrm{C}$, Injector temp $=200{ }^{\circ} \mathrm{C}$, Detector temp $=250{ }^{\circ} \mathrm{C}$, Inlet pressure $\left.=75 \mathrm{kPa}\right), \mathrm{t}_{\mathrm{r}}($ major $)=22.64$ min, $\mathrm{t}_{\mathrm{r}}$ $($ minor $)=24.27 \mathrm{~min}$. $]$ gave the isomeric composition of the product: $89 \%$ ee; $[\alpha]_{\mathrm{D}}{ }^{25}=-21.1(\mathrm{c}=0.38$, $\left.\mathrm{CHCl}_{3}\right) ;{ }^{1} \mathrm{H}$ NMR $\left(400 \mathrm{MHz}, \mathrm{CDCl}_{3}\right): \delta$ 7.56-7.54 (m, 2H), 7.42-7.35 (m, 3H), $1.86(\mathrm{~s}, 3 \mathrm{H}), 0.18(\mathrm{~s}$, $9 \mathrm{H}) ;{ }^{13} \mathrm{C} \mathrm{NMR}\left(100 \mathrm{MHz}, \mathrm{CDCl}_{3}\right): \delta 142.08,128.73,128.71,124.70,121.73,71.70,33.65,1.13$.

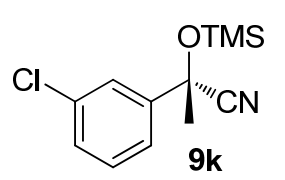

The reaction with $8 \mathbf{k}$ was conducted on a $0.5 \mathrm{mmol}$ scale at $-40{ }^{\circ} \mathrm{C}$ for 3 days; Column chromatography afforded $9 \mathbf{k}$ in $96 \%$ yield as colorless oil ${ }^{23}$ HPLC analysis (Chiralcel OJ-H+OJ-H, ${ }^{i} \mathrm{PrOH} /$ hexane $=0.5 / 95.5,0.4 \mathrm{~mL} / \mathrm{min}, 205 \mathrm{~nm}$; $\mathrm{t}_{\mathrm{r}}$ $($ major $)=22.71 \mathrm{~min}, \mathrm{t}_{\mathrm{r}}($ minor $\left.)=23.65 \mathrm{~min}\right)$ gave the isomeric composition of the product: $89 \%$ ee; $[\alpha]_{\mathrm{D}}{ }^{25}=-20.4\left(\mathrm{c}=1.27, \mathrm{CHCl}_{3}\right) ;{ }^{1} \mathrm{H} \mathrm{NMR}\left(400 \mathrm{MHz}, \mathrm{CDCl}_{3}\right): \delta 7.52(\mathrm{~s}, 1 \mathrm{H}), 7.52-7.42(\mathrm{~m}, 1 \mathrm{H})$, 7.36-7.33 (s, 2H), 1.84 (s, 3H), 0.21 (s, 9H); $\left.{ }^{13} \mathrm{C} \mathrm{NMR} \mathrm{(100} \mathrm{MHz,} \mathrm{CDCl}_{3}\right): \delta 144.25,134.80,130.06$, $128.95,125.03,122.91,121.22,71.09,33.59,1.15$;

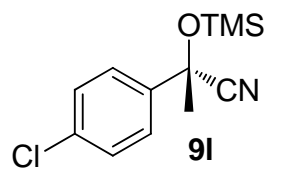

The reaction with 81 was conducted on a $0.5 \mathrm{mmol}$ scale at $-30{ }^{\circ} \mathrm{C}$ for 2 days; Column chromatography afforded 91 in $99 \%$ yield as colorless oil ${ }^{23}$ Chiral GC analysis [Agilent, Chirasil DEX CB $25 \mathrm{~m} \times 0.25 \mathrm{~mm} \times 0.25 \mu \mathrm{m}$, column temp $=$ $120{ }^{\circ} \mathrm{C}$, Injector temp $=200{ }^{\circ} \mathrm{C}$, Detector temp $=250{ }^{\circ} \mathrm{C}$, Inlet pressure $\left.=75 \mathrm{kPa}\right), \mathrm{t}_{\mathrm{r}}$ (major) $=27.65$ $\min , \mathrm{t}_{\mathrm{r}}($ minor $)=29.32 \mathrm{~min}$.] gave the isomeric composition of the product: $92 \%$ ee; $[\alpha]_{\mathrm{D}}{ }^{25}=-20.3(\mathrm{c}$ $\left.=1.08, \mathrm{CHCl}_{3}\right) ;{ }^{1} \mathrm{H} \mathrm{NMR}\left(400 \mathrm{MHz}, \mathrm{CDCl}_{3}\right): \delta 7.48(\mathrm{~d}, J=8.8 \mathrm{~Hz}, 2 \mathrm{H}), 7.37(\mathrm{~d}, J=8.8 \mathrm{~Hz}, 2 \mathrm{H})$, $1.83(\mathrm{~s}, 3 \mathrm{H}), 0.19$ (s, 9H); ${ }^{13} \mathrm{C}$ NMR (100 MHz, $\left.\mathrm{CDCl}_{3}\right): \delta 140.80,134.70,128.93,126.17,121.34$, $71.16,33.62,1.15$. 


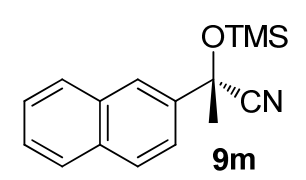

The reaction with $8 \mathrm{~m}$ was conducted on a $0.5 \mathrm{mmol}$ scale at $-30{ }^{\circ} \mathrm{C}$ for 3 days; Column chromatography afforded $\mathbf{9 m}$ in $99 \%$ yield as colorless oil; ${ }^{24}$ HPLC analysis $\left(\right.$ Chiralcel OJ-H, ${ }^{i} \mathrm{PrOH} /$ hexane $=1 / 99,1.0 \mathrm{~mL} / \mathrm{min}, 230 \mathrm{~nm}$; $\mathrm{t}_{\mathrm{r}}$ (major) $=$ $7.59 \mathrm{~min}, \mathrm{t}_{\mathrm{r}}($ minor $\left.)=6.10 \mathrm{~min}\right)$ gave the isomeric composition of the product: $90 \%$ ee; $[\alpha]_{\mathrm{D}}{ }^{25}=-11.3$ $\left(\mathrm{c}=1.15, \mathrm{CHCl}_{3}\right) ;{ }^{1} \mathrm{H}$ NMR $\left(400 \mathrm{MHz}, \mathrm{CDCl}_{3}\right): \delta 8.04(\mathrm{~m}, 1 \mathrm{H}), 7.90-7.85(\mathrm{~m}, 3 \mathrm{H}), 7.62-7.60(\mathrm{~m}, 1 \mathrm{H})$, 7.54-7.52 (m, 2H), 1.94 (s, 3H), 0.19 (s, 9H); ${ }^{13} \mathrm{C}$ NMR (100 MHz, $\left.\mathrm{CDCl}_{3}\right): \delta$ 139.29, 133.29, 132.90, $128.84,128.46,127.74,126.81,126.77,123.79,122.44,121.73,71.92,33.61,1.20$.

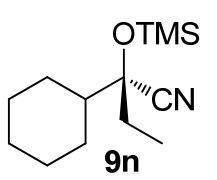

The reaction with $8 \mathrm{n}$ was conducted on a $1.0 \mathrm{mmol}$ scale at $-30{ }^{\circ} \mathrm{C}$ for 3 days; Column chromatography afforded 9n in 90\% yield as colorless oil; Chiral GC analysis [Agilent, Chirasil DEX CB $25 \mathrm{~m} \times 0.25 \mathrm{~mm} \times 0.25 \mu \mathrm{m}$, column temp $=110{ }^{\circ} \mathrm{C}$, Injector temp $=$ $200{ }^{\circ} \mathrm{C}$, Detector temp $=250{ }^{\circ} \mathrm{C}$, Inlet pressure $\left.=60 \mathrm{kPa}\right), \mathrm{t}_{\mathrm{r}}($ major $)=33.56 \mathrm{~min}, \mathrm{t}_{\mathrm{r}}($ minor $)=35.75$ min.] gave the isomeric composition of the product: $95 \%$ ee; $[\alpha]_{\mathrm{D}}{ }^{25}=-7.8\left(\mathrm{c}=1.04, \mathrm{CHCl}_{3}\right) ;{ }^{1} \mathrm{H} \mathrm{NMR}$ (400 MHz, $\left.\mathrm{CDCl}_{3}\right): \delta 1.88-1.75(\mathrm{~m}, 6 \mathrm{H}), 1.69-1.67(\mathrm{~m}, 1 \mathrm{H}), 1.56-1.54(\mathrm{~m}, 1 \mathrm{H}), 1.25-1.09(\mathrm{~m}, 5 \mathrm{H})$, $1.00(\mathrm{t}, J=7.2 \mathrm{~Hz}, 3 \mathrm{H}), 0.23(\mathrm{~s}, 9 \mathrm{H}) ;{ }^{13} \mathrm{C} \mathrm{NMR}\left(100 \mathrm{MHz}, \mathrm{CDCl}_{3}\right): \delta 121.40,45.28,30.98,27.37$, 26.78, 26.21, 26.14, 8.13, 1.40; IR (KBr) v 2935.0, 2856.6, 1453.2, 1253.2, 1104.6, 903.8, 871.3, $845.3 \mathrm{~cm}^{-1}$; MS (EI): 239 (M+, 2.6), 197 (16), 182 (3.7), 157 (100), 130 (24), 115 (7.7), 100 (29), 73 (25); HRMS (EI): Exact mass calcd for $\mathrm{C}_{13} \mathrm{H}_{23} \mathrm{NOSi}$ : 239.1705, found: 239.1704.

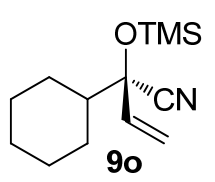

The reaction with 80 was conducted on a $1.0 \mathrm{mmol}$ scale at $-40{ }^{\circ} \mathrm{C}$ for 4 days; Column chromatography afforded 90 in $92 \%$ yield as colorless oil; The enantiomeric excess of 9o was determined after transforming to the corresponding acetate 9oa; $[\alpha]_{\mathrm{D}}{ }^{25}=-9.4$ (c $\left.=1.10, \mathrm{CHCl}_{3}\right) ;{ }^{1} \mathrm{H}$ NMR $\left(400 \mathrm{MHz}, \mathrm{CDCl}_{3}\right): \delta$ 5.77-5.70 (m, $\left.1 \mathrm{H}\right), 5.58-5.54(\mathrm{~m}, 1 \mathrm{H}), 5.35-5.33(\mathrm{~m}$, $1 \mathrm{H}), 2.02-1.98(\mathrm{~d}, J=13.2 \mathrm{~Hz}, 1 \mathrm{H}), 1.83-1.68(\mathrm{~m}, 3 \mathrm{H}), 1.68-1.66(\mathrm{~m}, 1 \mathrm{H}), 1.58-1.51(\mathrm{~m}, 1 \mathrm{H})$, 1.18-1.04 (m, 5H), 0.20 (s, 9H); $\left.{ }^{13} \mathrm{C} \mathrm{NMR} \mathrm{(100} \mathrm{MHz,} \mathrm{CDCl}_{3}\right): \delta 137.11,119.31,117.55,78.10,48.23$, 27.20, 26.99, 26.17, 26.05, 26.00, 1.36; IR (KBr) v 2932.4, 2856.9, 1452.5, 1253.3, 1198.0, 904.42, 871.0, $846.3 \mathrm{~cm}^{-1}$; MS (EI): 237 (M+, 0.68), 222 (10), 195 (22), 155 (100), 140 (21), 128 (50), 113 (72), 100 (32); HRMS (EI): Exact mass calcd for $\mathrm{C}_{13} \mathrm{H}_{23} \mathrm{NOSi}$ : 237.1549, found: 237.1552.

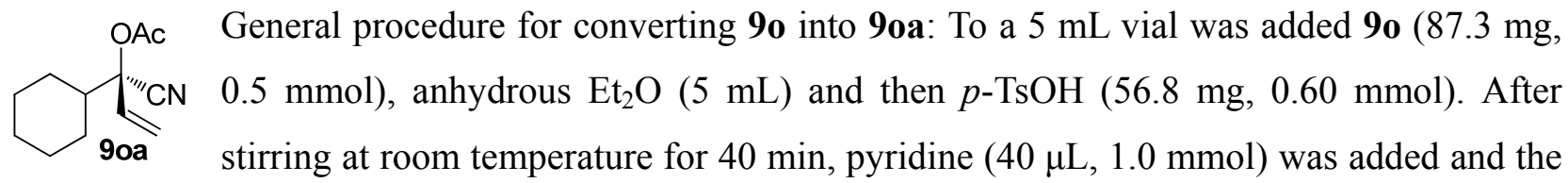
solution was filtrated. The filtrate was evaporated under reduced pressure and dried under vacuum to give the crude free cyanohydrin, which was dissolved in anhydrous $\mathrm{CH}_{2} \mathrm{Cl}_{2}(2 \mathrm{~mL})$ and cooled to $0{ }^{\circ} \mathrm{C}$, 
pyridine $(48 \mu \mathrm{L} 1.0 \mathrm{mmol})$ and acetal chloride $(43 \mu \mathrm{L}, 0.6 \mathrm{mmol})$ were then added. The resulting solution was stirred for about at rt till full conversion of the free cyanohydrin indicated by TLC analysis, and then $\mathrm{NaHCO}_{3}$ (sat.) was added to quench the reaction. Usual work-up procedure and column chromatography afforded 90 in $67 \%$ yield as colorless oil; Chiral GC analysis [Agilent, Chirasil DEX CB $25 \mathrm{~m} \times 0.25 \mathrm{~mm} \times 0.25 \mu \mathrm{m}$, column temp $=115{ }^{\circ} \mathrm{C}$, Injector temp $=200{ }^{\circ} \mathrm{C}$, Detector temp $=250{ }^{\circ} \mathrm{C}$, Inlet pressure $\left.=75 \mathrm{kPa}\right), \mathrm{t}_{\mathrm{r}}($ major $)=41.07 \mathrm{~min}, \mathrm{t}_{\mathrm{r}}($ minor $)=42.64 \mathrm{~min}$.] gave the isomeric composition of the product: $80 \%$ ee; $[\alpha]_{\mathrm{D}}{ }^{25}=-26.3\left(\mathrm{c}=0.69, \mathrm{CHCl}_{3}\right) ;{ }^{1} \mathrm{H} \mathrm{NMR}$ $\left(400 \mathrm{MHz}, \mathrm{CDCl}_{3}\right): \delta 5.73-5.63(\mathrm{~m}, 2 \mathrm{H}), 5.49(\mathrm{dd}, J=7.9,2.8 \mathrm{~Hz}, 1 \mathrm{H}) .2 .09$ (s, 3H), 2.02-2.00 (m, $1 \mathrm{H}), 1.88-1.78(\mathrm{~m}, 4 \mathrm{H}), 1.72-1.69(\mathrm{~m}, 1 \mathrm{H}), 1.28-1.17(\mathrm{~m}, 5 \mathrm{H}) ;{ }^{13} \mathrm{C} \mathrm{NMR}\left(100 \mathrm{MHz}, \mathrm{CDCl}_{3}\right): \delta 168.46$, 132.43, 120.17, 116.04, 79.38, 45.67, 27.09, 26.75, 25.95, 25.86, 25.77, 21.10; IR (KBr) v 2934.5, 2858.0, 1759.5, 1412.8, 1370.5, 1223.4, 1020.0, $995.8 \mathrm{~cm}^{-1}$; MS (EI): 207 (M+, 0.15), 165 (15), 126 (6), 125 (88), 83 (100), 67 (7), 55 (96), 41 (26); HRMS (EI): Exact mass calcd for $\mathrm{C}_{12} \mathrm{H}_{17} \mathrm{NO}_{2}$ : 207.1259, found: 207.1261 .

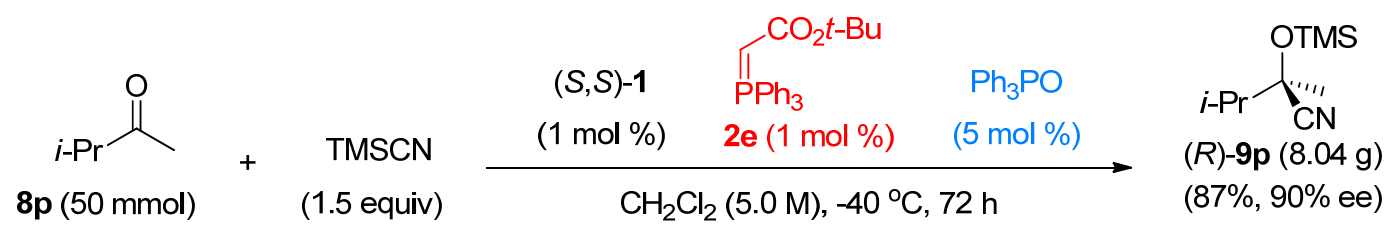

The reaction with $8 \mathbf{p}$ was conducted on a $50 \mathrm{mmol}$ scale at $-40{ }^{\circ} \mathrm{C}$ for $72 \mathrm{~h}$ followed the general procedure; Reduced pressure distillation afforded $\mathbf{9} \mathbf{p}^{25}$ in $87 \%$ yield as colorless oil; Chiral GC analysis [Agilent, Chirasil DEX CB $25 \mathrm{~m} \times 0.25 \mathrm{~mm} \times 0.25 \mu \mathrm{m}$, column temp $=50{ }^{\circ} \mathrm{C}$, Injector temp $=200{ }^{\circ} \mathrm{C}$, Detector temp $=250{ }^{\circ} \mathrm{C}$, Inlet pressure $\left.=75 \mathrm{kPa}\right), \mathrm{t}_{\mathrm{r}}($ major $)=33.61 \mathrm{~min}, \mathrm{t}_{\mathrm{r}}($ minor $)=36.39$ min.] gave the isomeric composition of the product: $90 \%$ ee; $[\alpha]_{\mathrm{D}}{ }^{25}=+11.6\left(\mathrm{c}=1.0, \mathrm{CHCl}_{3}\right) ;{ }^{1} \mathrm{H}$ NMR (400 MHz, $\left.\mathrm{CDCl}_{3}\right): \delta 1.88-1.81(\mathrm{~m}, 1 \mathrm{H}), 1.52(\mathrm{~s}, 3 \mathrm{H}), 1.04-1.01(\mathrm{ABd}, J=4.4 \mathrm{~Hz}, 6 \mathrm{H}), 0.23(\mathrm{~s}$, $9 \mathrm{H}) ;{ }^{13} \mathrm{C} \mathrm{NMR}\left(100 \mathrm{MHz}, \mathrm{CDCl}_{3}\right): \delta 121.73,73.61,39.22,26.11,17.26,17.06,1.31$. 


\section{Tandem Wittig-cyanosilylation sequence using $\alpha, \beta$-unsaturated enals 10}
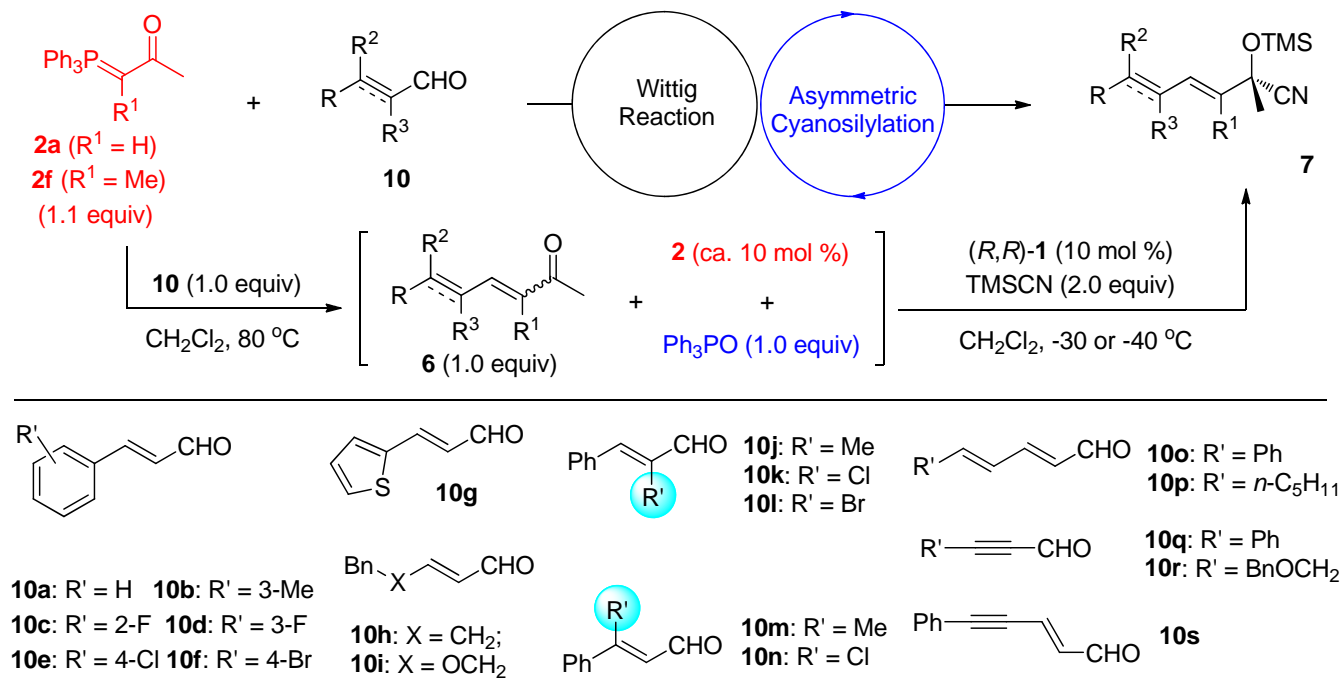

\begin{tabular}{|c|c|c|c|c|c|c|c|}
\hline Entry & 10 & 2 & 7 & $\mathrm{~T}_{1}(\mathrm{~h})$ & $\mathrm{T}_{2}(\mathrm{~h})$ & Isolated yield (\%) & $\operatorname{Ee}(\%)^{\mathrm{a}}$ \\
\hline 1 & $10 \mathbf{a}$ & $2 a$ & $7 \mathbf{a}$ & 25 & 48 & 70 & 91 \\
\hline 2 & $10 \mathrm{~b}$ & $2 a$ & $7 \mathbf{b}$ & 25 & 48 & 71 & 92 \\
\hline 3 & $10 \mathrm{c}$ & $2 a$ & $7 \mathrm{c}$ & 18 & 36 & 82 & 93 \\
\hline 4 & $10 d$ & $2 a$ & $7 d$ & 21 & 48 & 81 & 90 \\
\hline 5 & $10 \mathrm{e}$ & $2 a$ & $7 e$ & 15 & 48 & 77 & 91 \\
\hline 6 & $10 f$ & $2 a$ & $7 f$ & 16 & 48 & 80 & 92 \\
\hline 7 & $10 \mathrm{~g}$ & $2 \mathbf{a}$ & $7 \mathrm{~g}$ & 19 & 48 & 80 & 92 \\
\hline 8 & $10 \mathrm{~h}$ & $2 a$ & $7 \mathbf{h}$ & 21 & 60 & 60 & 90 \\
\hline 9 & $10 \mathbf{i}$ & $2 a$ & $7 \mathbf{i}$ & 21 & 120 & 42 & 90 \\
\hline 10 & $\mathbf{1 0 j}$ & $2 \mathbf{a}$ & $7 \mathbf{j}$ & 21 & 48 & 77 & 91 \\
\hline 11 & $10 \mathrm{k}^{\mathrm{b}}$ & $2 \mathbf{a}$ & $7 \mathbf{k}$ & 1 & 24 & 95 & 92 \\
\hline 12 & $101^{b}$ & $2 a$ & 71 & 1 & 16 & 96 & 92 \\
\hline 13 & $10 \mathrm{~m}$ & $2 a$ & $7 \mathrm{~m}$ & 17 & 48 & 72 & 80 \\
\hline 14 & $10 n$ & $2 a$ & $7 n$ & 12 & 14 & 67 & 80 \\
\hline 15 & $101^{b}$ & $2 c$ & 70 & $50 \mathrm{~min}$ & 6 & 98 & 84 \\
\hline 16 & 100 & $2 a$ & $7 p$ & 21 & 60 & 75 & 91 \\
\hline 17 & $10 p$ & $2 a$ & $7 q$ & 24 & 72 & 60 & 92 \\
\hline 18 & $10 q$ & $2 a$ & $7 r$ & $10 \mathrm{~min}$ & 24 & 95 & 83 \\
\hline 19 & $10 r$ & $2 a$ & $7 s$ & $20 \mathrm{~min}$ & 24 & 91 & 81 \\
\hline 20 & $10 \mathrm{~s}$ & $2 a$ & $7 t$ & 4 & 26 & 88 & 90 \\
\hline
\end{tabular}

${ }^{a}$ Determined by chiral HPLC analysis. ${ }^{b}$ Conducted at $-40{ }^{\circ} \mathrm{C}$ for step 2 . 
General procedure: To a screw-capped pressure tube were added anhydrous $\mathrm{CH}_{2} \mathrm{Cl}_{2}(1.0 \mathrm{~mL})$, $\alpha, \beta$-unsaturated enal $10(0.5 \mathrm{mmol})$, and phosphorane $2(175.0 \mathrm{mg}, 0.55 \mathrm{mmol})$. Then the tube was sealed and heated to $80{ }^{\circ} \mathrm{C}$ until the full consumption of $\mathbf{1 0}$ indicated by TLC analysis. After the reaction mixture was cooled to room temperature (rt), (salen)AlCl $(R, R)-\mathbf{1}(30.3 \mathrm{mg}, 0.05 \mathrm{mmol})$ was added. The resulting solution was cooled to -30 or $-40{ }^{\circ} \mathrm{C}$ (as indicated above) and stirred for half an hour before the addition of TMSCN $(1.0 \mathrm{mmol})$. After TLC analysis indicated the full conversion of the trans-6 (cis-6 was unreactive in step 2 in most cases), the mixture was directly subjected for column chromatograph, and product 7 was isolated using petroleum ether/diethyl ether (from 100:0 to $10: 1)$ as the eluent.

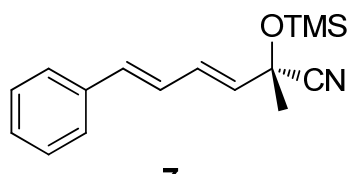

$7 a$

The reaction with $\mathbf{1 0 a}$ was conducted for 25 hours for step 1 and 2 days for step 2 at $-30{ }^{\circ} \mathrm{C}$; Column chromatography afforded $7 \mathbf{a}$ in $70 \%$ yield as pale yellow oil; HPLC analysis (Chiralcel OJ-H, ${ }^{i} \mathrm{PrOH} / \mathrm{hexane}=1 / 99,1.0 \mathrm{~mL} / \mathrm{min}$, $230 \mathrm{~nm}$; $\mathrm{t}_{\mathrm{r}}($ major $)=16.67 \mathrm{~min}, \mathrm{t}_{\mathrm{r}}($ minor $\left.)=13.64 \mathrm{~min}\right)$ gave the isomeric composition of the product: $91 \%$ ee; $[\alpha]_{\mathrm{D}}{ }^{25}=-31.2\left(\mathrm{c}=1.0, \mathrm{CHCl}_{3}\right) ;{ }^{1} \mathrm{H}$ NMR $\left(400 \mathrm{MHz}, \mathrm{CDCl}_{3}\right): \delta 7.43-7.41(\mathrm{~m}, 2 \mathrm{H}), 7.34(\mathrm{t}, J$ $=8.0 \mathrm{~Hz}, 2 \mathrm{H}), 7.28-7.24(\mathrm{~m}, 1 \mathrm{H}), 6.75-6.64(\mathrm{~m}, 3 \mathrm{H}), 5.74(\mathrm{~d}, J=14.8 \mathrm{~Hz}, 1 \mathrm{H}), 1.70(\mathrm{~s}, 3 \mathrm{H}), 0.24(\mathrm{~s}$, $9 \mathrm{H}) ;{ }^{13} \mathrm{C} \mathrm{NMR}\left(100 \mathrm{MHz}, \mathrm{CDCl}_{3}\right): \delta 136.66,135.68,132.96,131.43,128.79,128.27,126.71,126.49$, 120.80, 69.76, 30.90, 1.49; IR (ATR) v 3027.45, 2959.79, 1642.71, 1490.92, 1253.12, 1099.57, 989.31, 842.06, 747.14, 690.40 cm $\mathrm{cm}^{-1}$ MS (EI): $271\left(\mathrm{M}^{+}, 58\right), 256$ (66), 228 (62), 182 (24), 157 (100), 129 (51), 115 (21), 73 (92). HRMS (EI): Exact mass calcd for $\mathrm{C}_{16} \mathrm{H}_{21} \mathrm{NOSi}$ : 271.1392; found: 271.1394.<smiles>COC(C)(C#N)/C=C/C=C/c1cccc(C)c1</smiles>

7b

The reaction with 10b was conducted for 25 hours for step 1 and 2 days for step 2 at $-30{ }^{\circ} \mathrm{C}$; Column chromatography afforded $7 \mathbf{b}$ in $71 \%$ yield as pale yellow oil; HPLC analysis (Chiralcel OJ-H+OJ-H, ${ }^{i} \mathrm{PrOH} /$ hexane $=$ 1/99, $0.8 \mathrm{~mL} / \mathrm{min}, 230 \mathrm{~nm} ; \mathrm{t}_{\mathrm{r}}($ major $)=28.35 \mathrm{~min}, \mathrm{t}_{\mathrm{r}}($ minor $\left.)=31.67 \mathrm{~min}\right)$ gave the isomeric composition of the product: $92 \%$ ee, $[\alpha]_{\mathrm{D}}{ }^{25}=-30.0\left(\mathrm{c}=1.0, \mathrm{CHCl}_{3}\right) ;{ }^{1} \mathrm{H} \mathrm{NMR}\left(400 \mathrm{MHz}, \mathrm{CDCl}_{3}\right): \delta$ 7.26-7.22 (m, 3H), $7.08(\mathrm{~s}, 1 \mathrm{H}), 6.75-6.63(\mathrm{~m}, 3 \mathrm{H}), 5.73(\mathrm{~d}, J=15.2 \mathrm{~Hz}, 1 \mathrm{H}), 2.36(\mathrm{~s}, 3 \mathrm{H}), 1.70(\mathrm{~s}$, 3H), 0.24 (s, 9H); ${ }^{13} \mathrm{C}$ NMR (100 MHz, $\left.\mathrm{CDCl}_{3}\right): \delta 138.34,136.57,135.81,132.71,131.52,129.09$, $128.67,127.39,126.28,123.88,120.80,69.78,30.88,21.46,1.48$; IR (ATR) v 2959.3, 1641.8, 1253.1, 1195.5, 1099.7, 988.1, 842.2, 756.0, $690.8 \mathrm{~cm}^{-1}$; MS (EI): 285 (M+, 61), 270 (77), 242 (51), 196 (29), 
172 (97), 143 (76), 128 (36), 73 (100). HRMS (EI): Exact mass calcd for $\mathrm{C}_{17} \mathrm{H}_{23} \mathrm{NOSi}$ : 285.1549; found: 285.1553 .

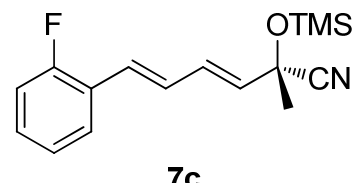

7c

The reaction with 10c was conducted for 18 hours for step 1 and 1.5 days for step 2 at $-30{ }^{\circ} \mathrm{C}$; Column chromatography afforded $7 \mathbf{c}$ in $82 \%$ yield as pale yellow oil; HPLC analysis (Chiralcel OJ-H, ${ }^{i} \mathrm{PrOH} /$ hexane $=1 / 99,1.0$ $\mathrm{mL} / \mathrm{min}, 230 \mathrm{~nm} ; \mathrm{t}_{\mathrm{r}}$ (major) $=8.73 \mathrm{~min}, \mathrm{t}_{\mathrm{r}}($ minor $\left.)=7.62 \mathrm{~min}\right)$ gave the isomeric composition of the product: $93 \%$ ee; $[\alpha]_{\mathrm{D}}{ }^{25}=-225.3\left(\mathrm{c}=1.0, \mathrm{CHCl}_{3}\right) ;{ }^{1} \mathrm{H} \mathrm{NMR}\left(400 \mathrm{MHz}, \mathrm{CDCl}_{3}\right): \delta 7.47(\mathrm{td}, J=7.8 \mathrm{~Hz}$, $1.6 \mathrm{~Hz}, 1 \mathrm{H}), 7.26-7.22(\mathrm{~m}, 1 \mathrm{H}), 7.13-7.05(\mathrm{~m}, 2 \mathrm{H}), 6.84-6.82(\mathrm{~m}, 2 \mathrm{H}), 6.71-6.64(\mathrm{~m}, 1 \mathrm{H}), 5.78(\mathrm{~d}, J=$ $15.2 \mathrm{~Hz}, 1 \mathrm{H}), 1.70(\mathrm{~s}, 3 \mathrm{H}), 0.25(\mathrm{~s}, 9 \mathrm{H}) ;{ }^{13} \mathrm{C} \mathrm{NMR}\left(100 \mathrm{MHz}, \mathrm{CDCl}_{3}\right): \delta 160.56(\mathrm{~d}, J=249 \mathrm{~Hz})$, 133.80, 131.54, 129.45 (d, $J=8.0 \mathrm{~Hz}), 129.01(\mathrm{~d}, J=6.0 \mathrm{~Hz}), 128.06$ (d, $J=3.0 \mathrm{~Hz}), 127.59$ (d, $J=$ $3.0 \mathrm{~Hz}), 124.58$ (d, $J=12.0 \mathrm{~Hz}), 124.29$ (d, $J=4.0 \mathrm{~Hz}), 120.73,115.99$ (d, $J=22.0 \mathrm{~Hz}), 69.65,30.84$, 1.47; ${ }^{19} \mathrm{~F}$ NMR (376 MHz, $\mathrm{CDCl}_{3}$ ): $\delta$-117.08; IR (ATR) v 2960.5, 1606.8, 1484.3, 1255.7, 1196.9, 1113.8, 1102.7, 994.4, 841.7, 748.1 $\mathrm{cm}^{-1}$; MS (EI): $289\left(\mathrm{M}^{+}, 54\right), 274$ (58), 246 (64), 190 (25), 175 (100), 147 (39), 133 (12), 73 (94); HRMS (EI): Exact mass calcd for $\mathrm{C}_{16} \mathrm{H}_{20} \mathrm{FNOSi}$ : 289.1298; found: 289.1296.

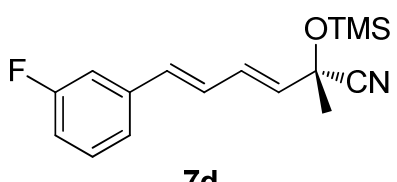

7d

The reaction with 10d was conducted for 21 hours for step 1 and 2 days for step 2 at $-30{ }^{\circ} \mathrm{C}$; Column chromatography afforded $7 \mathbf{d}$ in $81 \%$ yield as pale yellow oil; HPLC analysis (Chiralcel OJ-H, ${ }^{i} \mathrm{PrOH} /$ hexane $=1 / 99,1.0$ $\mathrm{mL} / \mathrm{min}, 230 \mathrm{~nm} ; \mathrm{t}_{\mathrm{r}}$ (major) $=13.70 \mathrm{~min}, \mathrm{t}_{\mathrm{r}}($ minor $\left.)=10.81 \mathrm{~min}\right)$ gave the isomeric composition of the product: $90 \%$ ee; $[\alpha]_{\mathrm{D}}{ }^{25}=-28.1\left(\mathrm{c}=1.0, \mathrm{CHCl}_{3}\right) ;{ }^{1} \mathrm{H} \mathrm{NMR}\left(400 \mathrm{MHz}, \mathrm{CDCl}_{3}\right): \delta 7.32-7.27(\mathrm{~m}, 1 \mathrm{H})$, $7.17(\mathrm{~d}, J=8.0 \mathrm{~Hz}, 1 \mathrm{H}), 7.12(\mathrm{~d}, J=10.0 \mathrm{~Hz}, 1 \mathrm{H}), 6.96(\mathrm{td}, J=8.3,2.0 \mathrm{~Hz}, 1 \mathrm{H}), 6.77-6.62(\mathrm{~m}, 3 \mathrm{H})$, $5.78(\mathrm{~d}, J=15.5 \mathrm{~Hz}, 1 \mathrm{H}), 1.70(\mathrm{~s}, 3 \mathrm{H}), 0.24(\mathrm{~s}, 9 \mathrm{H}) ;{ }^{13} \mathrm{C} \mathrm{NMR}\left(100 \mathrm{MHz}, \mathrm{CDCl}_{3}\right): \delta 163.17(\mathrm{~d}, J=$ $244 \mathrm{~Hz}), 138.99$ (d, $J=8.0 \mathrm{~Hz}), 134.27$ (d, $J=3.0 \mathrm{~Hz}), 133.99,130.88,130.21$ (d, $J=8.0 \mathrm{~Hz}), 127.76$, $122.67(\mathrm{~d}, J=3.0 \mathrm{~Hz}), 120.65,115.00$ (d, $J=21.0 \mathrm{~Hz}), 112.91$ (d, $J=22.0 \mathrm{~Hz}), 69.63,30.80,1.43$; ${ }^{19} \mathrm{~F} \mathrm{NMR}\left(376 \mathrm{MHz}, \mathrm{CDCl}_{3}\right): \delta$-113.25; IR (ATR) v 2963.8, 1581.5, 1447.3, 1252.5, 1197.2, 1145.9, 1099.2, 992.2, 841.0, 781.5, $754.3 \mathrm{~cm}^{-1}$; MS (EI): 289 (M+ , 43), 274 (61), 246 (59), 185 (23), 175 (87), 147 (46), 133 (14), 73 (94); HRMS (EI): Exact mass calcd for $\mathrm{C}_{16} \mathrm{H}_{20} \mathrm{FNOSi}$ : 289.1298; found: 289.1300. 


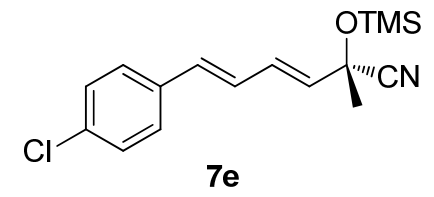

The reaction with 10e was conducted for 15 hours for step 1 and 2 days for step 2 at $-30{ }^{\circ} \mathrm{C}$; Column chromatography gave product $7 \mathrm{e}$ in $77 \%$ yield as pale yellow oil; HPLC analysis (Chiralcel OJ-H, ${ }^{i} \mathrm{PrOH} /$ hexane $=1 / 99,1.0$ $\mathrm{mL} / \mathrm{min}, 230 \mathrm{~nm} ; \mathrm{t}_{\mathrm{r}}($ major $)=15.33 \mathrm{~min}, \mathrm{t}_{\mathrm{r}}($ minor $\left.)=12.24 \mathrm{~min}\right)$ gave the isomeric composition of the product: $91 \%$ ee; $[\alpha]_{\mathrm{D}}{ }^{25}=-33.3\left(\mathrm{c}=1.0, \mathrm{CHCl}_{3}\right) ;{ }^{1} \mathrm{H} \mathrm{NMR}\left(400 \mathrm{MHz}, \mathrm{CDCl}_{3}\right): \delta 7.36-7.29(\mathrm{~m}, 4 \mathrm{H})$, 6.75- $6.62(\mathrm{~m}, 3 \mathrm{H}), 5.76(\mathrm{~d}, J=15.2 \mathrm{~Hz}, 1 \mathrm{H}), 1.70(\mathrm{~s}, 3 \mathrm{H}), 0.24(\mathrm{~s}, 9 \mathrm{H}) ;{ }^{13} \mathrm{C} \mathrm{NMR}\left(100 \mathrm{MHz}, \mathrm{CDCl}_{3}\right)$ : $\delta 135.15,134.24,133.87,133.57,131.06,128.97,127.84,127.06,120.70,69.68,30.85,1.47$; IR $(\mathrm{ATR})$ v 2959.8, 1643.0, 1490.8, 1253.6, 1195.9, 1094.3, 842.2, 756.5, 686.8 $\mathrm{cm}^{-1}$; MS (EI): $305\left(\mathrm{M}^{+}\right.$, 9), 290 (10), 262 (17), 206 (19), 191 (39), 171 (19), 128 (58), 73 (100). HRMS (EI): Exact mass calcd for $\mathrm{C}_{16} \mathrm{H}_{20}{ }^{35} \mathrm{CINOSi}$ : 305.1003 ; found: 305.1004 .

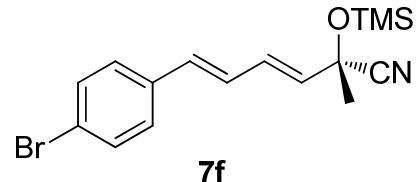

$7 f$

The reaction with $\mathbf{1 0 f}$ was conducted for 16 hours for step 1 and 2 days for step 2 at $-30{ }^{\circ} \mathrm{C}$; Column chromatography afforded $7 \mathbf{f}$ in $80 \%$ yield as pale yellow oil; HPLC analysis (Chiralcel OJ-H, ${ }^{i} \mathrm{PrOH} / \mathrm{h}$ exane $=1 / 99,1.0$ $\mathrm{mL} / \mathrm{min}, 230 \mathrm{~nm} ; \mathrm{t}_{\mathrm{r}}$ (major) $=17.08 \mathrm{~min}, \mathrm{t}_{\mathrm{r}}($ minor $\left.)=13.97 \mathrm{~min}\right)$ gave the isomeric composition of the product: $92 \%$ ee; $[\alpha]_{\mathrm{D}}{ }^{25}=-30.2\left(\mathrm{c}=1.0, \mathrm{CHCl}_{3}\right) ;{ }^{1} \mathrm{H}$ NMR $\left(400 \mathrm{MHz}, \mathrm{CDCl}_{3}\right): \delta 7.46(\mathrm{ABd}, J=8.4$ $\mathrm{Hz}, 2 \mathrm{H}), 7.28$ (ABd, $J=8.4 \mathrm{~Hz}, 2 \mathrm{H}), 6.77-6.60(\mathrm{~m}, 3 \mathrm{H}), 5.77$ (d, $J=14.8 \mathrm{~Hz}, 1 \mathrm{H}), 1.70(\mathrm{~s}, 3 \mathrm{H}), 0.24$ $(\mathrm{s}, 9 \mathrm{H}) ;{ }^{13} \mathrm{C} \mathrm{NMR}\left(100 \mathrm{MHz}, \mathrm{CDCl}_{3}\right): \delta 135.60,134.30,133.68,131.93,131.05,128.14,127.17$, 122.06, 120.69, 69.69, 30.85, 1.47; IR (ATR) v 2958.8, 1644.1, 1487.0, 1245.0, 1193.3, 1114.9, 836.8, 753.4, $705.3 \mathrm{~cm}^{-1}$; MS (EI): $349\left(\mathrm{M}^{+}, 28\right), 351(\mathrm{M}+2,28), 306$ (28), 308 (29), 270 (19), 237 (44), 180 (42), 128 (35), 73 (100); HRMS (EI): Exact mass calcd for $\mathrm{C}_{16} \mathrm{H}_{20}{ }^{79} \mathrm{BrNOSi}$ : 349.0498; found: 349.0501 .

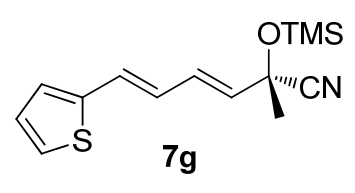

The reaction with $\mathbf{1 0 g}$ was conducted for 19 hours for step 1 and 2 days for step 2 at $-30{ }^{\circ} \mathrm{C}$; Column chromatography afforded $7 \mathbf{g}$ in $80 \%$ yield as pale yellow oil; HPLC analysis (Chiralcel OJ-H, ${ }^{i} \mathrm{PrOH} /$ hexane $=1 / 99,1.0 \mathrm{~mL} / \mathrm{min}$, $230 \mathrm{~nm} ; \mathrm{t}_{\mathrm{r}}($ major $)=18.13 \mathrm{~min}, \mathrm{t}_{\mathrm{r}}($ minor $\left.)=12.56 \mathrm{~min}\right)$ gave the isomeric composition of the product: $92 \%$ ee; $[\alpha]_{\mathrm{D}}{ }^{25}=-40.1\left(\mathrm{c}=1.0, \mathrm{CHCl}_{3}\right) ;{ }^{1} \mathrm{H} \mathrm{NMR}\left(400 \mathrm{MHz}, \mathrm{CDCl}_{3}\right): \delta$ 7.22-7.20 (m, $\left.1 \mathrm{H}\right), 7.03-6.98$ $(\mathrm{m}, 2 \mathrm{H}), 6.84-6.81(\mathrm{~m}, 1 \mathrm{H}), 6.64-6.52(\mathrm{~m}, 2 \mathrm{H}), 5.73-5.69(\mathrm{~m}, 1 \mathrm{H}), 1.69(\mathrm{~s}, 3 \mathrm{H}), 0.23(\mathrm{~s}, 9 \mathrm{H}) ;{ }^{13} \mathrm{C}$ NMR (100 MHz, $\left.\mathrm{CDCl}_{3}\right): \delta 141.96,132.70,130.94,128.39,127.78,127.01,126.08,125.30,120.74$, 69.73, 30.89, 1.47; IR (ATR) v 2961.6, 1637.4, 1429.2, 1254.3, 1182.4, 1100.5, 987.8, 839.5, 757.6, $694.2 \mathrm{~cm}^{-1}$; MS (EI): 277 (M+, 63), 262 (78), 234 (51), 188 (39), 163 (96), 135 (48), 91 (17), 73 (100); HRMS (EI): Exact mass calcd for $\mathrm{C}_{14} \mathrm{H}_{19} \mathrm{NOSSi}$ : 277.0957; found: 277.0961 . 


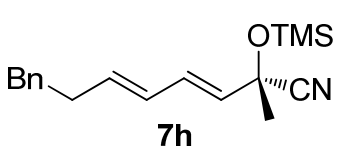

$7 \mathrm{~h}$

The reaction with $10 \mathrm{~h}$ was conducted for 21 hours for step 1 and 2.5 days for step 2 at $-30{ }^{\circ} \mathrm{C}$; Product $7 \mathbf{h}$ was obtained in $60 \%$ yield as pale yellow oil; HPLC analysis (Chiralcel OJ-H+OJ-H, ${ }^{i} \mathrm{PrOH} / \mathrm{hexane}=1 / 99,0.8 \mathrm{~mL} / \mathrm{min}, 230$ $\mathrm{nm} ; \mathrm{t}_{\mathrm{r}}($ major $)=18.47 \mathrm{~min}, \mathrm{t}_{\mathrm{r}}($ minor $\left.)=20.76 \mathrm{~min}\right)$ gave the isomeric composition of the product: $90 \%$ ee; $[\alpha]_{\mathrm{D}}{ }^{25}=-6.2\left(\mathrm{c}=1.0, \mathrm{CHCl}_{3}\right) ;{ }^{1} \mathrm{H}$ NMR $\left(400 \mathrm{MHz}, \mathrm{CDCl}_{3}\right): \delta 7.31-7.26(\mathrm{~m}, 2 \mathrm{H}) 7.21-7.17$ (m, 3H), 6.45 (dd, $J=15.3 \mathrm{~Hz}, 10.2 \mathrm{~Hz}, 1 \mathrm{H}), 6.07-6.00$ (m, 1H), 5.92-5.85 (m, 1H), 5.51 (d, $J=15.3$ $\mathrm{Hz}, 1 \mathrm{H}), 2.73(\mathrm{t}, J=8.0 \mathrm{~Hz}, 2 \mathrm{H}), 2.44(\mathrm{dd}, \mathrm{J}=14.9,7.3 \mathrm{~Hz}, 2 \mathrm{H}), 1.65(\mathrm{~s}, 3 \mathrm{H}), 0.21(\mathrm{~s}, 9 \mathrm{H}) ;{ }^{13} \mathrm{C} \mathrm{NMR}$ $\left(100 \mathrm{MHz}, \mathrm{CDCl}_{3}\right): \delta 141.48,137.50,131.39,130.77,128.60,128.49,128.44,126.03,120.88,69.76$, 35.48, 34.61, 30.85, 1.46; IR (ATR) v 3027.0, 2957.7, 1657.1, 1253.1, 1100.0, 990.6, 842.2, 749.6, $698.2 \mathrm{~cm}^{-1}$; HRMS (ESI): Exact mass calcd for $\left(\mathrm{C}_{18} \mathrm{H}_{25} \mathrm{NOSi}+\mathrm{NH}_{4}{ }^{+}\right): 317.2044$; found: 317.2041 .

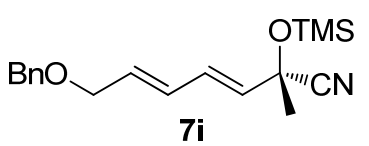

The reaction with 10i was conducted for 21 hours for step 1 and 5 days for step 2 at $-30{ }^{\circ} \mathrm{C}$; Column chromatography afforded $7 \mathbf{i}$ in $42 \%$ yield as pale yellow oil; HPLC analysis (Chiralcel OJ-H+OJ-H, ${ }^{i} \mathrm{PrOH} /$ hexane $=1 / 99,0.8$ $\mathrm{mL} / \mathrm{min}, 230 \mathrm{~nm} ; \mathrm{t}_{\mathrm{r}}$ (major) $=57.18 \mathrm{~min}, \mathrm{t}_{\mathrm{r}}($ minor $\left.)=62.20 \mathrm{~min}\right)$ gave the isomeric composition of the product: $90 \%$ ee; $[\alpha]_{\mathrm{D}}{ }^{25}=-5.6\left(\mathrm{c}=1.0, \mathrm{CHCl}_{3}\right) ;{ }^{1} \mathrm{H} \mathrm{NMR}\left(400 \mathrm{MHz}, \mathrm{CDCl}_{3}\right): \delta 7.36-7.26(\mathrm{~m}, 5 \mathrm{H})$, 6.55-6.48 (m, 1H), 6.31-6.25 (m, 1H), 5.99-5.93 (m, 1H), 5.65-5.62 (m, 1H), 4.54 (s, 2H), 4.10 (dd, $J$ $=5.6,1.0 \mathrm{~Hz}, 2 \mathrm{H}), 1.66(\mathrm{~s}, 3 \mathrm{H}), 0.22(\mathrm{~s}, 9 \mathrm{H}) ;{ }^{13} \mathrm{C} \mathrm{NMR}\left(100 \mathrm{MHz}, \mathrm{CDCl}_{3}\right): \delta 138.10,133.22,132.95$, $130.47,129.81,128.51,127.83,127.79,120.71,72.47,69.96,69.61,30.82,1.44$; IR (ATR) v 2955.8, 2853.2, 1660.7, 1496.5, 1253.5, 1101.6, 991.1, 842.5, 736.0, $697.3 \mathrm{~cm}^{-1}$; HRMS (ESI): Exact mass calcd for $\left(\mathrm{C}_{18} \mathrm{H}_{25} \mathrm{NO}_{2} \mathrm{Si}+\mathrm{NH}_{4}{ }^{+}\right)$: 333.1993; found: 333.1991 .

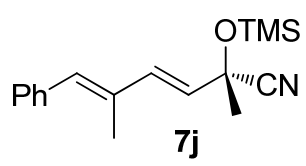

The reaction with $\mathbf{1 0 j}$ was conducted for 21 hours for step 1 and 2 days for step 2 at $-30{ }^{\circ} \mathrm{C}$; Product $7 \mathbf{j}$ was obtained in $77 \%$ yield as pale yellow oil; HPLC analysis (Chiralcel OJ-H+OJ-H, ${ }^{i} \mathrm{PrOH} /$ hexane $=1 / 99,0.8 \mathrm{~mL} / \mathrm{min}, 230 \mathrm{~nm} ; \mathrm{t}_{\mathrm{r}}$ $($ major $)=17.42 \mathrm{~min}, \mathrm{t}_{\mathrm{r}}($ minor $\left.)=16.28 \mathrm{~min}\right)$ gave the isomeric composition of the product: $91 \%$ ee; $[\alpha]_{\mathrm{D}}{ }^{25}=-8.8\left(\mathrm{c}=1.0, \mathrm{CHCl}_{3}\right) ;{ }^{1} \mathrm{H}$ NMR $\left(400 \mathrm{MHz}, \mathrm{CDCl}_{3}\right): \delta$ 7.38-7.23 (m, 5H), 6.72-6.66 (m, 2H), $5.70(\mathrm{~d}, J=15.6 \mathrm{~Hz}, 1 \mathrm{H}), 2.00(\mathrm{~s}, 3 \mathrm{H}), 1.72(\mathrm{~s}, 3 \mathrm{H}), 0.24(\mathrm{~s}, 9 \mathrm{H}) ;{ }^{13} \mathrm{C} \mathrm{NMR}\left(100 \mathrm{MHz}, \mathrm{CDCl}_{3}\right): \delta$ $137.25,136.31,134.34,133.67,129.33,128.84,128.30,127.15,121.00,70.10,31.14,14.03,1.53$; IR $(\mathrm{ATR})$ v 2955.0, 1635.8, 1493.0, 1253.2, 1104.1, 999.3, 842.7, 750.9, $696.0 \mathrm{~cm}^{-1}$; MS (EI): $285\left(\mathrm{M}^{+}\right.$, 20), 270 (22), 242 (18), 171 (50), 143 (100), 128 (29), 91 (8), 75 (17), 73 (64). HRMS (EI): Exact mass calcd for $\mathrm{C}_{17} \mathrm{H}_{23} \mathrm{NOSi}$ : 285.1549; found: 285.1551 . 


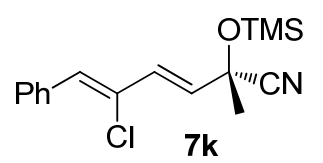

The reaction with $\mathbf{1 0 k}$ was conducted for 1 hour for step 1 and 1 day for step 2 at $-40{ }^{\circ} \mathrm{C}$; Column chromatography afforded $7 \mathbf{k}$ in $95 \%$ yield as pale yellow oil; HPLC analysis (Chiralcel OJ-H, ${ }^{i} \mathrm{PrOH} / \mathrm{hexane}=1 / 99,1.0 \mathrm{~mL} / \mathrm{min}, 230 \mathrm{~nm}$; $\mathrm{t}_{\mathrm{r}}$ $($ major $)=10.74 \mathrm{~min}, \mathrm{t}_{\mathrm{r}}($ minor $\left.)=8.98 \mathrm{~min}\right)$ gave the isomeric composition of the product: $92 \%$ ee; $[\alpha]_{\mathrm{D}}{ }^{25}=-21.8\left(\mathrm{c}=1.0, \mathrm{CHCl}_{3}\right) ;{ }^{1} \mathrm{H}$ NMR $\left(400 \mathrm{MHz}, \mathrm{CDCl}_{3}\right): \delta 7.73-7.71(\mathrm{~m}, 2 \mathrm{H}), 7.41-7.38(\mathrm{~m}, 2 \mathrm{H})$, 7.34-7.32 (m, 1H), 6.80 (s, 1H), $6.72(\mathrm{ABd}, J=15.2 \mathrm{~Hz}, 1 \mathrm{H}), 6.17$ (ABd, $J=14.8 \mathrm{~Hz}, 1 \mathrm{H}), 1.74$ (s, $3 \mathrm{H}), 0.25$ (s, 9H); ${ }^{13} \mathrm{C}$ NMR (100 MHz, $\left.\mathrm{CDCl}_{3}\right): \delta 134.37,132.70,131.59,130.74,129.86,128.78$, 128.46, 120.66, 69.52, 30.98, 1.46; IR (ATR) v 2960.6, 1640.9, 1494.2, 1288.9, 1103.8, 998.3, 841.8, 754.2, $690.7 \mathrm{~cm}^{-1}$; MS (EI): $305\left(\mathrm{M}^{+}, 30\right), 270$ (36), 262 (32), 171 (50), 143 (53), 128 (29), 93 (15), 75 (22), 73 (100); HRMS (EI): Exact mass calcd for $\mathrm{C}_{16} \mathrm{H}_{20}{ }^{35}$ CINOSi: 305.1003 ; found: 305.1005 .

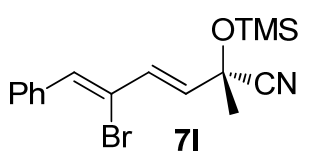

The reaction with $\mathbf{1 0 I}$ was conducted for 1 hour for step 1 and 16 hours for step 2 at $-40{ }^{\circ} \mathrm{C}$; Column chromatography afforded 71 in $96 \%$ yield as pale yellow oil; HPLC analysis (Chiralcel OJ-H, ${ }^{i} \mathrm{PrOH} /$ hexane $=1 / 99,1.0 \mathrm{~mL} / \mathrm{min}, 230 \mathrm{~nm} ; \mathrm{t}_{\mathrm{r}}$ $($ major $)=12.48 \mathrm{~min}, \mathrm{t}_{\mathrm{r}}($ minor $\left.)=10.02 \mathrm{~min}\right)$ gave the isomeric composition of the product: $92 \%$ ee; $[\alpha]_{\mathrm{D}}{ }^{25}=-18.1\left(\mathrm{c}=1.0, \mathrm{CHCl}_{3}\right) ;{ }^{1} \mathrm{H}$ NMR $\left(400 \mathrm{MHz}, \mathrm{CDCl}_{3}\right): \delta 7.72-7.70(\mathrm{~m}, 2 \mathrm{H}), 7.41-7.34(\mathrm{~m}, 3 \mathrm{H})$, $7.11(\mathrm{~s}, 1 \mathrm{H}), 6.70(\mathrm{ABd}, J=14.8 \mathrm{~Hz}, 1 \mathrm{H}), 6.18(\mathrm{ABd}, J=14.8 \mathrm{~Hz}, 1 \mathrm{H}), 1.74(\mathrm{~s}, 3 \mathrm{H}), 0.26$ (s, 9H); ${ }^{13} \mathrm{C}$ NMR $\left(100 \mathrm{MHz}, \mathrm{CDCl}_{3}\right): \delta 135.18,134.90,134.75,131.94,129.72,128.83,128.34,120.75$, 120.65, 69.47, 31.03, 1.48; IR (ATR) v 2967.2, 1637.55, 1492.7, 1289.5, 1096.6, 991.6, 839.4, 753.7, $692.9 \mathrm{~cm}^{-1}$; MS (EI): 349 (M+16), 351 (M+2, 16), 270 (56), 155 (57), 180 (66), 143 (62), 128 (47), 73 (100); HRMS (EI): Exact mass calcd for $\mathrm{C}_{16} \mathrm{H}_{20}{ }^{79}$ BrNOSi: 349.0498 ; found: 349.0497 .

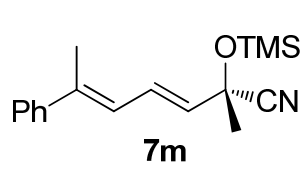

The reaction with $10 \mathrm{~m}$ was conducted for 17 hours for step 1 and 2 days for step 2 at $-30{ }^{\circ} \mathrm{C}$; Column chromatography afforded $7 \mathbf{m}$ in $72 \%$ yield as pale yellow oil; HPLC analysis (Chiralcel OJ-H, ${ }^{i} \mathrm{PrOH} /$ hexane $=1 / 99,1.0 \mathrm{~mL} / \mathrm{min}, 230 \mathrm{~nm} ; \mathrm{t}_{\mathrm{r}}$ $($ major $)=8.92 \mathrm{~min}, \mathrm{t}_{\mathrm{r}}($ minor $\left.)=12.33 \mathrm{~min}\right)$ gave the isomeric composition of the product: $80 \%$ ee; $[\alpha]_{\mathrm{D}}{ }^{25}=-21.9\left(\mathrm{c}=1.0, \mathrm{CHCl}_{3}\right) ;{ }^{1} \mathrm{H} \mathrm{NMR}\left(400 \mathrm{MHz}, \mathrm{CDCl}_{3}\right): \delta$ 7.47-7.45 (m, 2H), 7.37-7.34 (m, 2H), 7.30-7.28 (m, 1H), $6.92(\mathrm{dd}, J=15.1,11.0 \mathrm{~Hz}, 1 \mathrm{H}), 6.42(\mathrm{~d}, J=11.0 \mathrm{~Hz}, 1 \mathrm{H}), 5.73(\mathrm{~d}, J=15.1 \mathrm{~Hz}$, $1 \mathrm{H}), 2.23(\mathrm{~s}, 3 \mathrm{H}), 1.71(\mathrm{~s}, 3 \mathrm{H}), 0.24(\mathrm{~s}, 9 \mathrm{H}) ;{ }^{13} \mathrm{C} \mathrm{NMR}\left(100 \mathrm{MHz}, \mathrm{CDCl}_{3}\right): \delta 142.57,140.03,133.04$, $128.45,127.75,127.72,125.82,124.61,120.92,69.96,30.98,16.39,1.52$; IR (ATR) v 2956.3, 1638.9, 1445.1, 1252.9, 1101.0, 997.6, 842.8, 756.6, $693.7 \mathrm{~cm}^{-1}$; MS (EI): $285\left(\mathrm{M}^{+}, 38\right), 20$ (71), 242 (42), 195 (32), 180 (43), 143 (83), 128 (34), 73 (100); HRMS (EI): Exact mass calcd for $\mathrm{C}_{17} \mathrm{H}_{23} \mathrm{NOSi}$ : 285.1549; found: 285.1550 . 
<smiles>CO[C@](C)(C#N)/C=C/C=C(\Cl)c1ccccc1</smiles>

The reaction with 10n was conducted for 12 hours for step 1 and 14 hours for step 2 at $-30{ }^{\circ} \mathrm{C}$; Column chromatography afforded $\mathbf{7 n}$ in $67 \%$ yield as pale yellow oil; HPLC analysis (Chiralcel OJ-H, ${ }^{i} \mathrm{PrOH} /$ hexane $=1 / 99,1.0 \mathrm{~mL} / \mathrm{min}, 230 \mathrm{~nm} ; \mathrm{t}_{\mathrm{r}}$ $($ major $)=12.05 \mathrm{~min}, \mathrm{t}_{\mathrm{r}}($ minor $\left.)=17.09 \mathrm{~min}\right)$ gave the isomeric composition of the product: $80 \%$ ee; $[\alpha]_{\mathrm{D}}{ }^{25}=-24.0\left(\mathrm{c}=1.0, \mathrm{CHCl}_{3}\right) ;{ }^{1} \mathrm{H} \mathrm{NMR}\left(400 \mathrm{MHz}, \mathrm{CDCl}_{3}\right): \delta$ 7.66-7.64 (m, 2H), 7.41-7.36 (m, 3H), 7.07 (dd, $J=15.3,10.3 \mathrm{~Hz}, 1 \mathrm{H}), 6.72(\mathrm{~d}, J=9.2 \mathrm{~Hz}, 1 \mathrm{H}), 5.91$ (d, $J=15.3 \mathrm{~Hz}, 1 \mathrm{H}), 1.73(\mathrm{~s}, 3 \mathrm{H}), 0.26$ $(\mathrm{s}, 9 \mathrm{H}) ;{ }^{13} \mathrm{C} \mathrm{NMR}\left(100 \mathrm{MHz}, \mathrm{CDCl}_{3}\right): \delta 137.33,136.04,135.99,129.32,128.56,127.10,126.57$, 123.34, 120.57, 69.49, 30.68, 1.46; IR (ATR) v 2959.8, 1638.6, 1445.6, 1253.6, 1101.5, 997.1, 842.5, 757.5, $689.2 \mathrm{~cm}^{-1}$; HRMS (ESI): Exact mass calcd for $\left(\mathrm{C}_{16} \mathrm{H}_{20}{ }^{35} \mathrm{CINOSi}+\mathrm{Na}^{+}\right)$: 328.0895 ; found: 328.0891 .

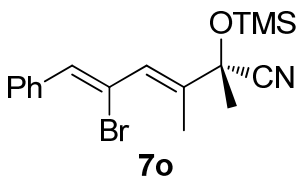

The reaction with $\mathbf{1 0 I}$ and $\mathbf{2 c}$ was conducted for 50 minutes for step 1 and 6 hours for step 2 at $-40{ }^{\circ} \mathrm{C}$; Column chromatography afforded 70 in $98 \%$ yield as pale yellow oil; HPLC analysis (Chiralpak IC-H+IC-H, ${ }^{i} \mathrm{PrOH} /$ hexane $=1 / 99,0.8$ $\mathrm{mL} / \mathrm{min}, 230 \mathrm{~nm} ; \mathrm{t}_{\mathrm{r}}$ (major) $=12.81 \mathrm{~min}, \mathrm{t}_{\mathrm{r}}($ minor $\left.)=12.25 \mathrm{~min}\right)$ gave the isomeric composition of the product: $84 \%$ ee; $[\alpha]_{\mathrm{D}}{ }^{25}=-4.1\left(\mathrm{c}=1.0, \mathrm{CHCl}_{3}\right) ;{ }^{1} \mathrm{H} \mathrm{NMR}\left(400 \mathrm{MHz}, \mathrm{CDCl}_{3}\right): \delta 7.66-7.64(\mathrm{~m}, 2 \mathrm{H})$, 7.41-7.33 (m, 3H), 6.78 (s, 1H), 6.57-6.56 (m, 1H), 1.99 (d, J=1.3 Hz, 3H), 1.73 (s, 3H), 0.29 (s, 9H); ${ }^{13} \mathrm{C}$ NMR $\left(100 \mathrm{MHz}, \mathrm{CDCl}_{3}\right): \delta 138.93,135.39,131.69,129.13,128.40,128.31,128.15,120.78$, 118.43, 72.59, 29.35, 13.66, 1.20; IR (ATR) v 2958.1, 1625.8, 1445.3, 1253.8, 1114.1, 991.9, 842.7, 751.0, $692.1 \mathrm{~cm}^{-1}$; MS (EI): $363\left(\mathrm{M}^{+}, 14\right), 355$ (M+2, 14), 284 (85), 269 (35), 194 (97), 185 (82), 115 (82), 73 (100); HRMS (EI): Exact mass calcd for $\mathrm{C}_{17} \mathrm{H}_{22}{ }^{79} \mathrm{BrNOSi}$ : 363.0654; found: 363.0657.

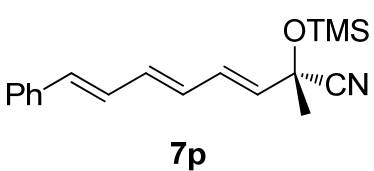

The reaction with $\mathbf{1 0 0}$ was conducted for 21 hours for step 1 and 2.5 days for step 2 at $-30{ }^{\circ} \mathrm{C}$; Column chromatography afforded $7 \mathbf{p}$ in $75 \%$ yield as pale yellow oil; HPLC analysis (Chiralcel OJ-H+OJ-H, ${ }^{i} \mathrm{PrOH} /$ hexane $=5 / 95,0.8$ $\mathrm{mL} / \mathrm{min}, 230 \mathrm{~nm} ; \mathrm{t}_{\mathrm{r}}($ major $)=20.26 \mathrm{~min}, \mathrm{t}_{\mathrm{r}}($ minor $\left.)=23.24 \mathrm{~min}\right)$ gave the isomeric composition of the product: $91 \%$ ee; $[\alpha]_{\mathrm{D}}{ }^{25}=-34.7\left(\mathrm{c}=1.0, \mathrm{CHCl}_{3}\right) ;{ }^{1} \mathrm{H} \mathrm{NMR}\left(400 \mathrm{MHz}, \mathrm{CDCl}_{3}\right): \delta 7.45-7.43(\mathrm{~m}, 2 \mathrm{H})$, $7.36(\mathrm{t}, J=8.0 \mathrm{~Hz}, 2 \mathrm{H}), 7.29-7.27(\mathrm{~m}, 1 \mathrm{H}), 6.89-6.82(\mathrm{~m}, 1 \mathrm{H}), 6.68-6.59(\mathrm{~m}, 3 \mathrm{H}), 6.37-6.31(\mathrm{~m}, 1 \mathrm{H})$, $5.69(\mathrm{~d}, J=15.2 \mathrm{~Hz}, 1 \mathrm{H}), 1.71(\mathrm{~s}, 3 \mathrm{H}), 0.26(\mathrm{~s}, 9 \mathrm{H}) ;{ }^{13} \mathrm{C} \mathrm{NMR}\left(100 \mathrm{MHz}, \mathrm{CDCl}_{3}\right): \delta 137.01,136.16$, 134.36, 132.64, 131.31, 130.47, 128.76, 128.32, 127.99, 126.60, 120.77, 69.76, 30.86, 1.47; IR (ATR) v 2967.1, 1665.1, 1448.0, 1254.0, 1188.0, 1119.0, 999.2, 842.9, 748.7, $688.9 \mathrm{~cm}^{-1}$; MS (EI): 297 (M , 54), 282 (27), 254 (21), 208 (25), 183 (30), 155 (100), 115 (22), 73 (82); HRMS (EI): Exact mass calcd for $\mathrm{C}_{18} \mathrm{H}_{23} \mathrm{NOSi}$ : 297.1549; found: 297.1546 . 


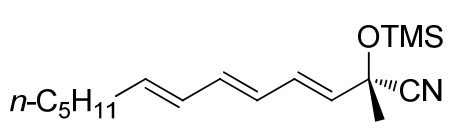

$7 q$

The reaction with $\mathbf{1 0 q}$ was conducted for 24 hours for step 1 and 3 days for step 2 at $-30{ }^{\circ} \mathrm{C}$; Column chromatography afforded $7 \mathbf{q}$ in $60 \%$ yield as pale yellow oil; The enantiomeric excess of $\mathbf{7 q}$ was determined after transforming into the corresponding acetate $7 \mathbf{q a} ;[\alpha]_{\mathrm{D}}{ }^{25}=-1.9\left(\mathrm{c}=1.0, \mathrm{CHCl}_{3}\right) ;{ }^{1} \mathrm{H} \mathrm{NMR}(400 \mathrm{MHz}$, $\left.\mathrm{CDCl}_{3}\right): \delta 6.51(\mathrm{dd}, J=15.3,10.6 \mathrm{~Hz}, 1 \mathrm{H}), 6.36-6.30(\mathrm{~m}, 1 \mathrm{H}), 6.11-6.04(\mathrm{~m}, 2 \mathrm{H}), 5.84-5.77(\mathrm{~m}, 1 \mathrm{H})$, $5.57(\mathrm{~d}, J=15.3 \mathrm{~Hz}, 1 \mathrm{H}), 2.11(\mathrm{q}, J=3.2 \mathrm{~Hz}, 2 \mathrm{H}) 1.66(\mathrm{~s}, 3 \mathrm{H}), 1.43-1.40(\mathrm{~m}, 2 \mathrm{H}), 1.30-1.29(\mathrm{~m}, 4 \mathrm{H})$, $0.89(\mathrm{t}, J=2.8 \mathrm{~Hz}, 3 \mathrm{H}), 0.20(\mathrm{~s}, 9 \mathrm{H}) ;{ }^{13} \mathrm{C} \mathrm{NMR}\left(100 \mathrm{MHz}, \mathrm{CDCl}_{3}\right): \delta 137.95,136.49,131.60,131.52$, $129.83,127.74,120.87,69.83,32.91,31.48,30.92,28.90,22.59,14.11,1.47$; IR (ATR) v 3019.4, 2958.4, 2928.4, 1634.9, 1456.8, 1253.7, 1101.2, 996.2, 842.7, 756.9, $691.7 \mathrm{~cm}^{-1}$; MS (EI): $291\left(\mathrm{M}^{+}\right.$, 40), 276 (36), 220 (45), 177 (30), 144 (13), 121 (21), 107 (34), 73 (100); HRMS (EI): Exact mass calcd for $\mathrm{C}_{17} \mathrm{H}_{29} \mathrm{NOSi}$ : 291.2018; found: 291.2025.

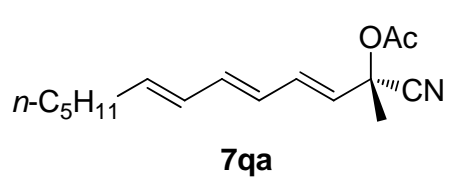

$7 q a$

The conversion of $\mathbf{7 q}$ to $\mathbf{7 q a}$ follows the general procedure for the conversion of 9n to 9na. Column chromatograph gave acetate $\mathbf{7 q a}$ as pale yellow oil in 65\% yield for two steps. HPLC analysis (Chiralcel $\mathrm{OJ}-\mathrm{H}+\mathrm{OJ}-\mathrm{H},{ }^{i} \mathrm{PrOH} / \mathrm{h}$ exane $=3 / 97,0.8 \mathrm{~mL} / \mathrm{min}, 230 \mathrm{~nm}$; $\mathrm{t}_{\mathrm{r}}$ (major) $=24.10 \mathrm{~min}, \mathrm{t}_{\mathrm{r}}($ minor $)=22.74$ min) gave the isomeric composition of the product: $92 \%$ ee; $[\alpha]_{\mathrm{D}}{ }^{25}=+34.8\left(\mathrm{c}=1.0, \mathrm{CHCl}_{3}\right)$; ${ }^{1} \mathrm{H} \mathrm{NMR}$ $\left(400 \mathrm{MHz}, \mathrm{CDCl}_{3}\right): \delta 6.63(\mathrm{dd}, J=15.3,10.7 \mathrm{~Hz}, 1 \mathrm{H}), 6.39-6.32(\mathrm{~m}, 1 \mathrm{H}), 6.10-6.03(\mathrm{~m}, 2 \mathrm{H})$, 5.86-5.80 (m, 1H), $5.62(\mathrm{~d}, J=15.3 \mathrm{~Hz}, 1 \mathrm{H}), 2.14-2.08(\mathrm{~m}, 5 \mathrm{H}), 1.82(\mathrm{~s}, 3 \mathrm{H}), 1.43-1.38(\mathrm{~m}, 2 \mathrm{H})$, $1.33-1.26(\mathrm{~m}, 4 \mathrm{H}), 0.88(\mathrm{t}, J=6.9 \mathrm{~Hz}, 3 \mathrm{H}) ;{ }^{13} \mathrm{C} \mathrm{NMR}\left(100 \mathrm{MHz}, \mathrm{CDCl}_{3}\right): \delta 168.64,138.63,137.61$, $134.65,129.72,127.33,126.41,117.74,71.34,32.91,31.46,28.83,26.66,22.58,21.21$, 14.11; IR $($ ATR $)$ v 3019.8, 2957.3, 2926.9, 2856.3, 1754.7, 1633.4, 1369.3, 1224.0, 1069.5, 996.7, 939.3 $\mathrm{cm}^{-1}$; MS (EI): 261 (M+, 71), 219 (38), 202 (54), 190 (31), 144 (100), 132 (92), 117 (59), 79 (45); HRMS (EI): Exact mass calcd for $\mathrm{C}_{16} \mathrm{H}_{23} \mathrm{NO}_{2}$ : 261.1729; found: 261.1728 .

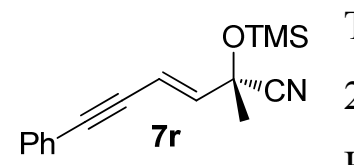

The reaction with $\mathbf{1 0 q}$ was conducted for 10 minutes for step 1 and 1 day for step 2 at $-30{ }^{\circ} \mathrm{C}$; Column chromatography afforded $\mathbf{7 r}$ in $95 \%$ yield as pale yellow oil; HPLC analysis (Chiralcel OJ-H+OJ-H, ${ }^{i} \mathrm{PrOH} /$ hexane $=1 / 99,0.8 \mathrm{~mL} / \mathrm{min}, 230$ $\mathrm{nm} ; \mathrm{t}_{\mathrm{r}}($ major $)=28.39 \mathrm{~min}, \mathrm{t}_{\mathrm{r}}($ minor $\left.)=25.09 \mathrm{~min}\right)$ gave the isomeric composition of the product: $83 \%$ ee; $[\alpha]_{\mathrm{D}}{ }^{25}=-39.4\left(\mathrm{c}=1.0, \mathrm{CHCl}_{3}\right) ;{ }^{1} \mathrm{H} \mathrm{NMR}\left(400 \mathrm{MHz}, \mathrm{CDCl}_{3}\right): \delta 7.46-7.44(\mathrm{~m}, 2 \mathrm{H}), 7.34-7.32$ (m, 3H), 6.25-6.12 (m, 2H), 1.70 (s, 3H), 0.26 (s, 9H); ${ }^{13} \mathrm{C}$ NMR (100 MHz, $\left.\mathrm{CDCl}_{3}\right): \delta$ 141.70, 131.74, 128.77, 128.48, 122.75, 120.09, 111.79, 92.71, 85.76, 69.24, 30.55, 1.37; IR (ATR) v 2960.6, 1629.6, 
1443.0, 1254.3, 1107.3, 989.7, 842.9, 754.4, $689.2 \mathrm{~cm}^{-1}$; MS (EI): 269 (M+ 52 ), 254 (38), 226 (57), 155 (90), 142 (36), 127 (22), 73 (100); HRMS (EI): Exact mass calcd for $\mathrm{C}_{16} \mathrm{H}_{19} \mathrm{NOSi}$ : 269.1236; found: 269.1240 .

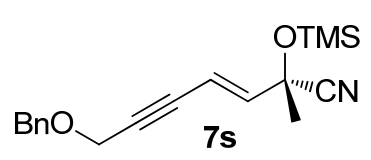

The reaction with 10r was conducted for 20 minutes for step 1 and 1 day for step 2 at $-30{ }^{\circ} \mathrm{C}$; Column chromatography afforded $7 \mathbf{s}$ in $91 \%$ yield as pale yellow oil; HPLC analysis (Chiralpak AS-H+AS-H, ${ }^{i} \mathrm{PrOH} /$ hexane $=1 / 99$, $0.8 \mathrm{~mL} / \mathrm{min}, 230 \mathrm{~nm} ; \mathrm{t}_{\mathrm{r}}$ (major) $=17.31 \mathrm{~min}, \mathrm{t}_{\mathrm{r}}($ minor $)=15.36 \mathrm{~min}$ ) gave the isomeric composition of the product: $81 \%$ ee; $[\alpha]_{\mathrm{D}}{ }^{25}=-25.4\left(\mathrm{c}=1.0, \mathrm{CHCl}_{3}\right) ;{ }^{1} \mathrm{H}$ NMR $\left(400 \mathrm{MHz}, \mathrm{CDCl}_{3}\right): \delta 7.37-7.31(\mathrm{~m}$, $5 \mathrm{H}), 6.10-6.01(\mathrm{~m}, 2 \mathrm{H}), 4.61(\mathrm{~s}, 2 \mathrm{H}), 4.30(\mathrm{~d}, J=1.2 \mathrm{~Hz}, 2 \mathrm{H}), 1.66(\mathrm{~s}, 3 \mathrm{H}), 0.25(\mathrm{~s}, 9 \mathrm{H}) ;{ }^{13} \mathrm{C} \mathrm{NMR}$ $\left(100 \mathrm{MHz}, \mathrm{CDCl}_{3}\right): \delta 142.44,137.35,128.55,128.18,128.03,119.97,111.13,88.63,82.82,71.85$, 69.09, 57.80, 30.43, 1.33; IR (ATR) v 2958.9, 1631.1, 1454.3, 1254.4, 1127.8, 996.7, 843.1, 737.9, $697.3 \mathrm{~cm}^{-1}$; HRMS (ESI): Exact mass calcd for $\left(\mathrm{C}_{18} \mathrm{H}_{23} \mathrm{NO}_{2} \mathrm{Si}+\mathrm{NH}_{4}{ }^{+}\right)$: 331.1836 ; found: 331.1836 .

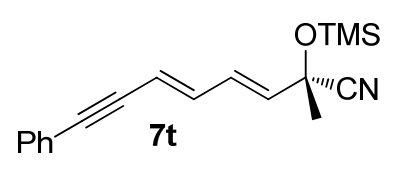

The reaction with 10s was conducted for 4 hours for step 1 and 26 hours for step 2 at $-30{ }^{\circ} \mathrm{C}$; Column chromatography afforded $\mathbf{7 t}$ in $88 \%$ yield as pale yellow oil; HPLC analysis (Chiralcel OJ-H+OJ-H, ${ }^{i} \mathrm{PrOH} /$ hexane $=1 / 99,0.8$ $\mathrm{mL} / \mathrm{min}, 230 \mathrm{~nm} ; \mathrm{t}_{\mathrm{r}}($ major $)=32.94 \mathrm{~min}, \mathrm{t}_{\mathrm{r}}($ minor $\left.)=28.65 \mathrm{~min}\right)$ gave the isomeric composition of the product: $90 \%$ ee; $[\alpha]_{\mathrm{D}}{ }^{25}=-48.3\left(\mathrm{c}=1.0, \mathrm{CHCl}_{3}\right) ;{ }^{1} \mathrm{H} \mathrm{NMR}\left(400 \mathrm{MHz}, \mathrm{CDCl}_{3}\right): \delta 7.46-7.43(\mathrm{~m}, 2 \mathrm{H})$ 7.34-7.31 (m, 3H), 6.68-6.54 (m, 2H), 6.00-5.96 (m, 1H), 5.77-5.73 (m, 1H), $1.69(\mathrm{~s}, 3 \mathrm{H}), 0.23(\mathrm{~s}, 9 \mathrm{H})$; ${ }^{13} \mathrm{C}$ NMR $\left(100 \mathrm{MHz}, \mathrm{CDCl}_{3}\right): \delta 139.01,134.92,131.65,130.34,128.56,128.46,123.14,120.52$, 114.76, 93.59, 88.26, 69.56, 30.76, 1.44; IR (ATR) v 2960.5, 1254.9, 1195.9, 1100.9, 985.7, 843.3, 754.9, $689.7 \mathrm{~cm}^{-1}$; MS (EI): 295 (M+28), 280 (25), 252 (18), 190 (26), 167 (12), 153 (32), 115 (5), 73 (100); HRMS (EI): Exact mass calcd for $\mathrm{C}_{18} \mathrm{H}_{21} \mathrm{NOSi}$ : 295.1392; found: 295.1391. 


\section{Tandem Wittig-cyanosilylation sequence using aliphatic aldehydes 3}

We first used enone 4a as the standard substrate to optimized the condition for the asymmetric cyanosilylation of $\alpha, \beta$-unsaturated ketones using chiral (salen)AlCl complex $(R, R)-\mathbf{1}$ and phosphorane 2a (10 mol \%, each), along with $100 \mathrm{~mol} \% \mathrm{Ph}_{3} \mathrm{PO}$. When using $\mathrm{CH}_{2} \mathrm{Cl}_{2}$ as the solvent, the asymmetric cyanosilylation of $\mathbf{4 a}$ afforded the desired product $\mathbf{5 a}$ in only $75 \%$ ee (entry 1, Table S6). Further optimization showed that DMF was a better solvent, allowing the desired product $\mathbf{5 a}$ to be obtained in up to $89 \%$ ee (entry 2). Noticeably, the use of ylide 2a to activate the $(R, R)-\mathbf{1}$ was still necessary to obtain high yield and enantioselectivity of the reaction, since only $17 \%$ yield with $85 \%$ ee for $\mathbf{5 a}$ was obtained in the absence of ylide $\mathbf{2 a}$ (entry 3 ).

Table S6. Condition optimization for asymmetric cyanosilylation of $\mathbf{4 a}$

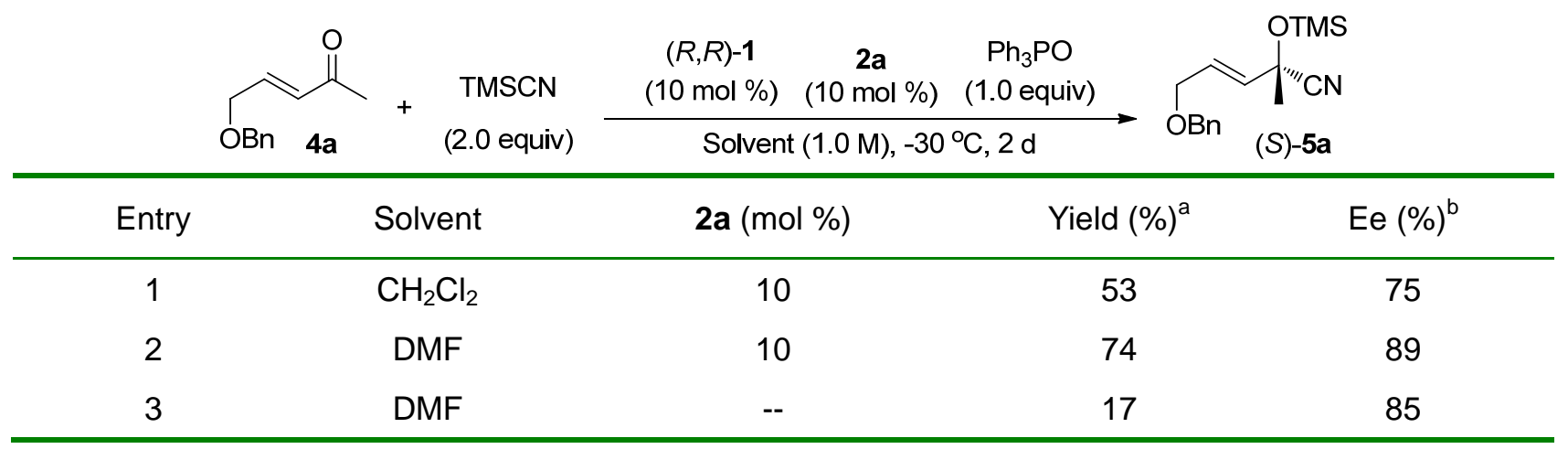

${ }^{\mathrm{a}}$ Isolated yield; ${ }^{\mathrm{b}}$ Determined by chiral HPLC analysis

We then optimized the condition for the tandem Wittig-cyanosilylation sequence, and found that when using DMF as the solvent for both steps, product 5a was obtained in low yield (entry 1, Table S7). So we conducted the Wittig step in $\mathrm{CH}_{2} \mathrm{Cl}_{2}$, and removed it before $(R, R)-\mathbf{1}$, TMSCN and DMF was added for the cyanosilylation, which give $\mathbf{5 a}$ in $71 \%$ yield and $90 \%$ ee (entry 2 ).

Table S7. Condition optimization for tandem Wittig-cyanosilylation of 3a

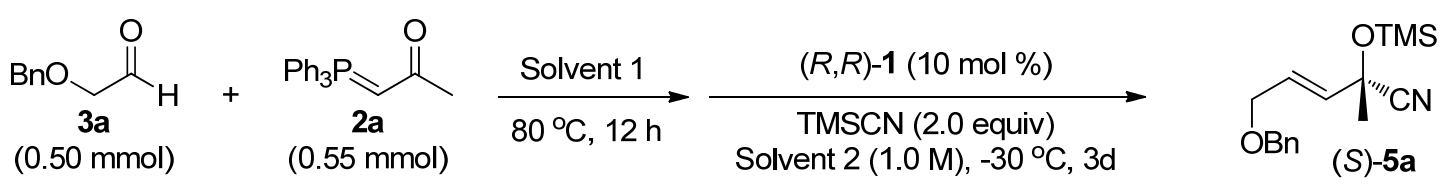

\begin{tabular}{ccccc}
\hline Entry & Solvent 1 & Solvent 2 & ${\text { Yield }(\%)^{a}}^{*}$ & Ee (\%) \\
\hline 1 & DMF & DMF & 7 & 85 \\
2 & $\mathrm{CH}_{2} \mathrm{Cl}_{2}$ & DMF & 71 & 90 \\
\hline
\end{tabular}

${ }^{a}$ Isolated yield; ${ }^{b}$ Determined by chiral HPLC analysis 


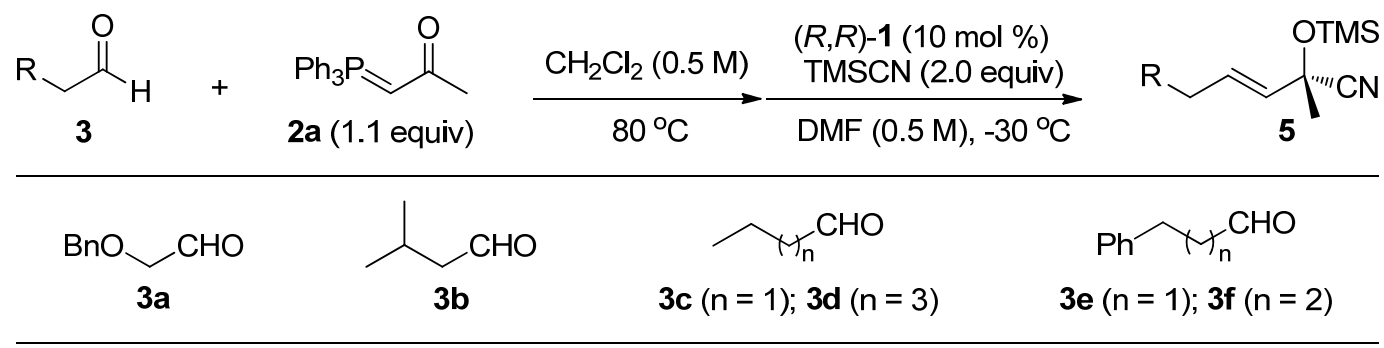

General procedure: To a screw-capped pressure tube were added anhydrous $\mathrm{CH}_{2} \mathrm{Cl}_{2}(1.0 \mathrm{~mL})$, aldehyde $3(0.50 \mathrm{mmol})$, and phosphorane $2 \mathbf{a}(175.0 \mathrm{mg}, 0.55 \mathrm{mmol})$. Then the tube was sealed and heated to $80{ }^{\circ} \mathrm{C}$ until the full consumption of 3 indicated by TLC analysis. The solvent $\mathrm{CH}_{2} \mathrm{Cl}_{2}$ was removed under reduced pressure and then DMF $(1.0 \mathrm{~mL})$ and $(R, R)-1(30.1 \mathrm{mg}, 0.05 \mathrm{mmol})$ were added. The resulting solution was stirred at $-30{ }^{\circ} \mathrm{C}$ for half an hour before TMSCN $(125 \mu \mathrm{L}, 1.0 \mathrm{mmol})$ was charged. After TLC analysis indicated the end of the reaction, the reaction mixture was poured into $\mathrm{NH}_{4} \mathrm{Cl}$ (sat.), after usual work-up procedure and column chromatography using $\mathrm{PE} / \mathrm{Et}_{2} \mathrm{O}(10: 0$ to10:1) as the eluent could afford the corresponding products 5 .

OTMS The reaction with 3a was conducted for 12 hours for step 1 and 3 days for step 2;
BnO (S)-5a analysis (Chiralcel OJ-H+OJ-H, ${ }^{i} \mathrm{PrOH} /$ hexane $=3 / 97,1.0 \mathrm{~mL} / \mathrm{min}, 205 \mathrm{~nm} ; \mathrm{t}_{\mathrm{r}}$ $($ major $)=16.92 \mathrm{~min}, \mathrm{t}_{\mathrm{r}}($ minor $\left.)=18.78 \mathrm{~min}\right)$ gave the isomeric composition of the product: $90 \%$ ee; $[\alpha]_{\mathrm{D}}{ }^{25}=-10.0\left(\mathrm{c}=1.0, \mathrm{CHCl}_{3}\right) ;{ }^{1} \mathrm{H}$ NMR $\left(400 \mathrm{MHz}, \mathrm{CDCl}_{3}\right): \delta 7.38-7.28(\mathrm{~m}, 5 \mathrm{H}), 6.11(\mathrm{dt}, J=15.5$, $5.0 \mathrm{~Hz}, 1 \mathrm{H}), 5.79(\mathrm{dt}, J=15.5,1.6 \mathrm{~Hz}, 1 \mathrm{H}), 4.55(\mathrm{~s}, 2 \mathrm{H}), 4.07(\mathrm{dd}, J=5.0,1.6 \mathrm{~Hz}, 2 \mathrm{H}), 1.66(\mathrm{~s}, 3 \mathrm{H})$, $0.23(\mathrm{~s}, 9 \mathrm{H}) ;{ }^{13} \mathrm{C}$ NMR $\left(100 \mathrm{MHz}, \mathrm{CDCl}_{3}\right): \delta 137.99,132.32,128.55,127.86,127.77,120.80,72.75$, $69.49,68.99,30.69,1.46$.

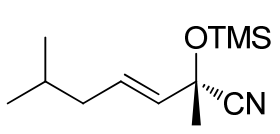

$5 b$

The reaction with $\mathbf{3 b}$ was conducted for 19 hours for step 1 and 2 days for step 2; Column chromatography afforded $\mathbf{5 b}$ in $75 \%$ yield as colorless oil; Chiral GC analysis [Agilent, Chirasil DEX CB $25 \mathrm{~m} \times 0.25 \mathrm{~mm} \times 0.25 \mu \mathrm{m}$, column temp $=90$ ${ }^{\circ} \mathrm{C}$, Injector temp $=200{ }^{\circ} \mathrm{C}$, Detector temp $=250{ }^{\circ} \mathrm{C}$, Inlet pressure $\left.=75 \mathrm{kPa}\right), \mathrm{t}_{\mathrm{r}}($ major $)=24.07 \mathrm{~min}, \mathrm{t}_{\mathrm{r}}$ $($ minor $)=23.66 \mathrm{~min}$.$] gave the isomeric composition of the product: 93 \%$ ee; $[\alpha]_{\mathrm{D}}^{25}=-0.7(\mathrm{c}=0.90$, $\left.\mathrm{CHCl}_{3}\right) ;{ }^{1} \mathrm{H} \mathrm{NMR}\left(400 \mathrm{MHz}, \mathrm{CDCl}_{3}\right): \delta 6.02-5.95(\mathrm{~m}, 1 \mathrm{H}), 5.46(\mathrm{dt}, J=15.6,1.2 \mathrm{~Hz}, 1 \mathrm{H}), 2.00-1.94$ $(\mathrm{m}, 2 \mathrm{H}), 1.74-1.66(\mathrm{~m}, 1 \mathrm{H}), 1.63(\mathrm{~s}, 3 \mathrm{H}), 0.92-0.89(\mathrm{~m}, 6 \mathrm{H}), 0.21(\mathrm{~s}, 9 \mathrm{H}) ;{ }^{13} \mathrm{C} \mathrm{NMR}(100 \mathrm{MHz}$, $\left.\mathrm{CDCl}_{3}\right): \delta 131.96,131.60,121.15,69.89,41.14,31.09,28.18,22.50,22.32,1.48 ; \mathrm{IR}(\mathrm{KBr}) v 2958.8$, 2359.7, 2342.0, 1254.0, 1111.9, 1001.6, 973.6, 757.1, $668.9 \mathrm{~cm}^{-1}$; MS (EI): 225 (M+, 0.78), 183 (11), 
154 (100), 127 (87), 129 (55), 75 (69), 73 (40), 55 (23); HRMS (EI): Exact mass calcd for $\mathrm{C}_{12} \mathrm{H}_{23} \mathrm{NOSi}: 225.1549$, found: 225.1551 .

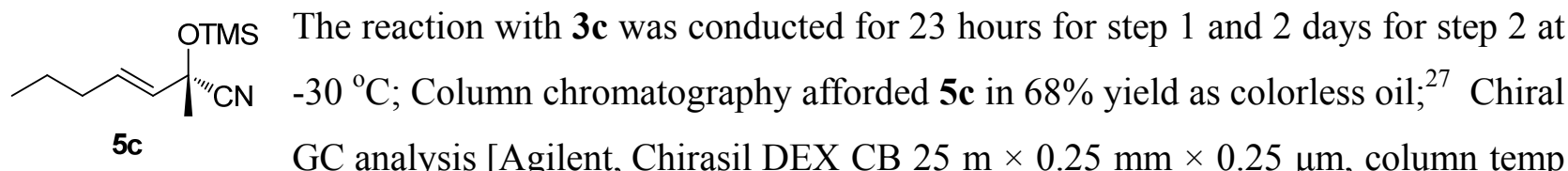
$=90{ }^{\circ} \mathrm{C}$, Injector temp $=200{ }^{\circ} \mathrm{C}$, Detector temp $=250{ }^{\circ} \mathrm{C}$, Inlet pressure $\left.=50 \mathrm{kPa}\right), \mathrm{t}_{\mathrm{r}}($ major $)=24.86 \mathrm{~min}$, $\mathrm{t}_{\mathrm{r}}($ minor $)=24.66 \mathrm{~min}$. $]$ gave the isomeric composition of the product: $95 \%$ ee; $[\alpha]_{\mathrm{D}}{ }^{25}=2.3(\mathrm{c}=1.0$, $\left.\mathrm{CHCl}_{3}\right) ;{ }^{1} \mathrm{H}$ NMR $\left(400 \mathrm{MHz}, \mathrm{CDCl}_{3}\right): \delta 6.01(\mathrm{dt}, J=15.2,4.2 \mathrm{~Hz}, 1 \mathrm{H}), 5.46(\mathrm{dt}, J=15.6,1.6 \mathrm{~Hz}, 1 \mathrm{H})$, $2.06(\mathrm{q}, J=7.4 \mathrm{~Hz}, 2 \mathrm{H}), 1.63(\mathrm{~s}, 3 \mathrm{H}), 1.47-1.40(\mathrm{~m}, 2 \mathrm{H}), 0.92(\mathrm{t}, J=7.4 \mathrm{~Hz}, 3 \mathrm{H}), 0.21(\mathrm{~s}, 9 \mathrm{H}) ;{ }^{13} \mathrm{C}$ $\operatorname{NMR}\left(100 \mathrm{MHz}, \mathrm{CDCl}_{3}\right): \delta 132.72,131.00,121.16,69.95,33.88,31.00,21.95,13.77,1.51$.

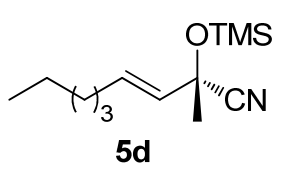

The reaction with 3d was conducted for 19 hours for step 1 and 2 days for step 2; Column chromatography afforded $\mathbf{5 d}$ in $56 \%$ yield as colorless oil ${ }^{21}$ Chiral GC analysis [Agilent, Chirasil DEX CB $25 \mathrm{~m} \times 0.25 \mathrm{~mm} \times 0.25 \mu \mathrm{m}$, column temp $=$ $100{ }^{\circ} \mathrm{C}$, Injector temp $=200{ }^{\circ} \mathrm{C}$, Detector temp $=250{ }^{\circ} \mathrm{C}$, Inlet pressure $\left.=75 \mathrm{kPa}\right), \mathrm{t}_{\mathrm{r}}$ (major) $=35.59 \mathrm{~min}$, $\mathrm{t}_{\mathrm{r}}($ minor $)=34.91 \mathrm{~min}$.] gave the isomeric composition of the product: $93 \%$ ee; $[\alpha]_{\mathrm{D}}{ }^{25}=-0.5(\mathrm{c}=1.0$, $\left.\mathrm{CHCl}_{3}\right) ;{ }^{1} \mathrm{H}$ NMR $\left(400 \mathrm{MHz}, \mathrm{CDCl}_{3}\right): \delta 6.01(\mathrm{dt}, J=15.2,2.8 \mathrm{~Hz}, 1 \mathrm{H}), 5.45(\mathrm{dt}, J=15.2,1.6 \mathrm{~Hz}, 1 \mathrm{H})$, 2.10-2.04 (m, 2H), 1.63 (s, 3H), 1.45-1.39 (m, 2H), 1.31-1.28 (m, 4H), 0.89 (t, $J=6.9 \mathrm{~Hz}, 3 \mathrm{H}), 0.21$ (s, 9H); ${ }^{13} \mathrm{C}$ NMR (100 MHz, $\left.\mathrm{CDCl}_{3}\right): \delta 132.98,130.78,121.16,69.96,31.80,31.47,30.98,28.40$, $22.53,14.07,1.51$.

OTMS The reaction with 3e was conducted for 19 hours for step 1 and 2 days for step 2;
ae $120{ }^{\circ} \mathrm{C}$, Injector temp $=200{ }^{\circ} \mathrm{C}$, Detector temp $=250{ }^{\circ} \mathrm{C}$, Inlet pressure $\left.=127 \mathrm{kPa}\right), \mathrm{t}_{\mathrm{r}}$ (major) $=73.05$ $\min , \mathrm{t}_{\mathrm{r}}($ minor $)=71.71 \mathrm{~min}$.] gave the isomeric composition of the product: $93 \%$ ee; $[\alpha]_{\mathrm{D}}{ }^{25}=-4.3(\mathrm{c}=$ 0.98, $\left.\mathrm{CHCl}_{3}\right) ;{ }^{1} \mathrm{H}$ NMR $\left(400 \mathrm{MHz}, \mathrm{CDCl}_{3}\right): \delta 7.30-7.27(\mathrm{~m}, 2 \mathrm{H}), 7.21-7.16(\mathrm{~m}, 3 \mathrm{H}), 6.08-6.04(\mathrm{dt}, J=$ 15.6, 4.2 Hz, 1H), 5.46 (dt, $J=15.6,1.2 \mathrm{~Hz}, 1 \mathrm{H}), 2.75-2.71(\mathrm{~m}, 2 \mathrm{H}), 2.42-2.39(\mathrm{~m}, 2 \mathrm{H}), 1.59$ (s, 3H), 0.19 (s, 9H); ${ }^{13} \mathrm{C}$ NMR $\left(100 \mathrm{MHz}, \mathrm{CDCl}_{3}\right): \delta 141.20,131.68,131.65,128.50,126.14,121.02,69.79$, 35.11, 33.63, 30.89, 1.47; IR (KBr) v 3028.1, 2958.7, 2858.6, 1496.8, 1253.9, 1109.6, 1003.4, 846.2, $699.5 \mathrm{~cm}^{-1}$; MS (EI): MS (EI): 273 (M+, 0.27), 231 (18), 168 (11), 156 (60), 141 (26), 91 (100), 75 (25), 73 (17); HRMS (EI): Exact mass calcd for $\mathrm{C}_{16} \mathrm{H}_{23} \mathrm{NOSi}$ : 273.1549, found: 273.1551. 
<smiles>COC(C)(C)/C=C/CCBr</smiles>

$5 f$

The reaction with $\mathbf{3 f}$ was conducted for 6 hours for step 1 and 2 days for step 2; Column chromatography afforded $\mathbf{5 f}$ in $84 \%$ yield as colorless oil; The enantiomeric excess of $\mathbf{5 f}$ was determined after the acetate $\mathbf{5 f a} ;[\alpha]_{\mathrm{D}}{ }^{25}=-2.8(\mathrm{c}=$ 1.02, $\mathrm{CHCl}_{3}$ ); ${ }^{1} \mathrm{H}$ NMR (400 MHz, $\left.\mathrm{CDCl}_{3}\right): \delta$ 7.30-7.27 (m, 2H), 7.21-7.16 (m, 3H), 6.04-5.99 (m, $1 \mathrm{H}), 5.49-5.45(\mathrm{~m}, 1 \mathrm{H}), 2.64(\mathrm{t}, J=7.7 \mathrm{~Hz}, 2 \mathrm{H}), 2.13-2.09(\mathrm{~m}, 2 \mathrm{H}), 1.79-1.71(\mathrm{~m}, 2 \mathrm{H}), 1.62(\mathrm{~s}, 3 \mathrm{H})$, 0.21 (s, 9H); ${ }^{13} \mathrm{C} \mathrm{NMR}\left(100 \mathrm{MHz}, \mathrm{CDCl}_{3}\right): \delta 142.00,132.31,131.33,128.49,128.45,125.95,121.09$, 69.86, 35.46, 31.32, 30.95, 30.40, 1.52; IR (KBr) v 3027.5, 2989.7, 2859.1, 1453.8, 1253.9, 1109.8, 1001.8, 971.6, $699.37 \mathrm{~cm}^{-1}$; MS (EI): 287 (M+, 1.1), 260 (18), 182 (21), 169 (74), 104 (100), 91 (61), 75 (68), 53 (36); HRMS (EI): Exact mass calcd for $\mathrm{C}_{17} \mathrm{H}_{25} \mathrm{NOSi}$ : 287.1705, found: 287.1709.

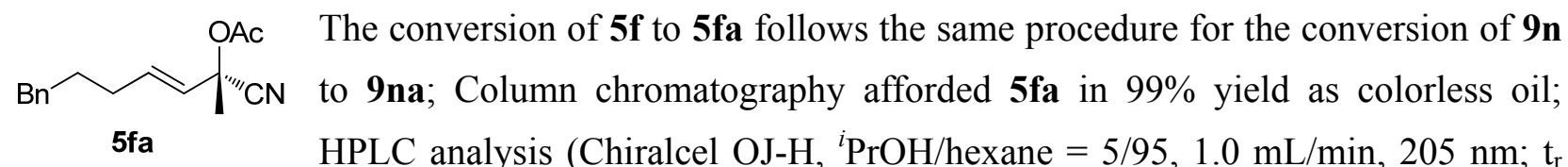
$($ major $)=18.52 \mathrm{~min}, \mathrm{t}_{\mathrm{r}}($ minor $\left.)=22.01 \mathrm{~min}\right)$ gave the isomeric composition of the product: $90 \%$ ee; $[\alpha]_{\mathrm{D}}{ }^{25}=-6.0\left(\mathrm{c}=0.87, \mathrm{CHCl}_{3}\right) ;{ }^{1} \mathrm{H} \mathrm{NMR}\left(400 \mathrm{MHz}, \mathrm{CDCl}_{3}\right): \delta 7.30-7.25(\mathrm{~m}, 2 \mathrm{H}), 7.20-7.15(\mathrm{~m}, 3 \mathrm{H})$, 6.19-6.16 (dt, $J=15.6,6.8 \mathrm{~Hz}, 1 \mathrm{H}), 5.52$ (d, $J=15.5 \mathrm{~Hz}, 1 \mathrm{H}), 2.62(\mathrm{t}, J=7.6 \mathrm{~Hz}, 2 \mathrm{H}), 2.14(\mathrm{q}, J=$ $7.1 \mathrm{~Hz}, 2 \mathrm{H}), 2.08$ (s, 3H), 1.79-1.72 (m, 5H); $\left.{ }^{13} \mathrm{C} \mathrm{NMR} \mathrm{(100} \mathrm{MHz,} \mathrm{CDCl}_{3}\right): \delta 168.58,141.90,135.78$, $128.49,128.41,126.71,125.92,117.87,71.30,35.24,31.31,30.15,26.69,21.20$; IR (KBr) v 3026.3, 2935.3, 2858.8, 1755.5, 1370.0, 1226.2, 1074.7, 965.0, $700.5 \mathrm{~cm}^{-1}$; MS (EI): 257 (M+, 0.04), 215 (4.76), 197 (6.6), 169 (3.2), 117 (6.3), 104 (100), 97 (4.3), 91 (54); HRMS (ESI): Exact mass calcd for $\left[\mathrm{C}_{16} \mathrm{H}_{19} \mathrm{NO}_{2}+\mathrm{Na}^{+}\right]: 280.1313$, found: 280.1315 .

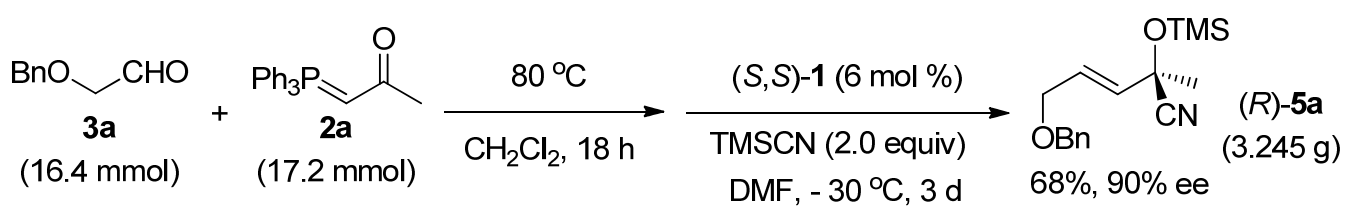

The reaction was conducted following the general procedure using 3a (2.46 g, $16.4 \mathrm{mmol})$, 2a (5.47 g, $17.2 \mathrm{mmol})$ in $\mathrm{CH}_{2} \mathrm{Cl}_{2}(16 \mathrm{~mL})$ for step 1; After the full consumption of $\mathbf{3 a}$ was observed by TLC analysis, the solvent $\mathrm{CH}_{2} \mathrm{Cl}_{2}$ was removed and then DMF (32 mL), $(S, S)-1$ (594.8 mg, 0.98 mmol) and TMSCN (4.1 mL, $33.0 \mathrm{mmol})$ were sequentially added; Column chromatography afforded $(R)-5 \mathrm{a}$ as colorless oil in $68 \%$ yield $(3.245 \mathrm{~g}) ;{ }^{28}$ HPLC analysis (Chiralcel OJ-H+OJ-H, ${ }^{i} \mathrm{PrOH} /$ hexane $=3 / 97,1.0 \mathrm{~mL} / \mathrm{min}, 205 \mathrm{~nm} ; \mathrm{t}_{\mathrm{r}}($ major $)=18.54 \mathrm{~min}, \mathrm{t}_{\mathrm{r}}($ minor $\left.)=16.78 \mathrm{~min}\right)$ gave the isomeric composition of the product: $90 \%$ ee; $[\alpha]_{\mathrm{D}}{ }^{25}=+9.7\left(\mathrm{c}=1.0, \mathrm{CHCl}_{3}\right)$ [Literature report $(R)-\mathbf{5 a}$ $(86 \%$ ee $\left.):[\alpha]{ }_{\mathrm{D}}^{21}=+4.4\left(\mathrm{c}=0.228, \mathrm{CHCl}_{3}\right)\right]$. 


\section{Elaboration and configuration determination of $7 \mathrm{a}$}

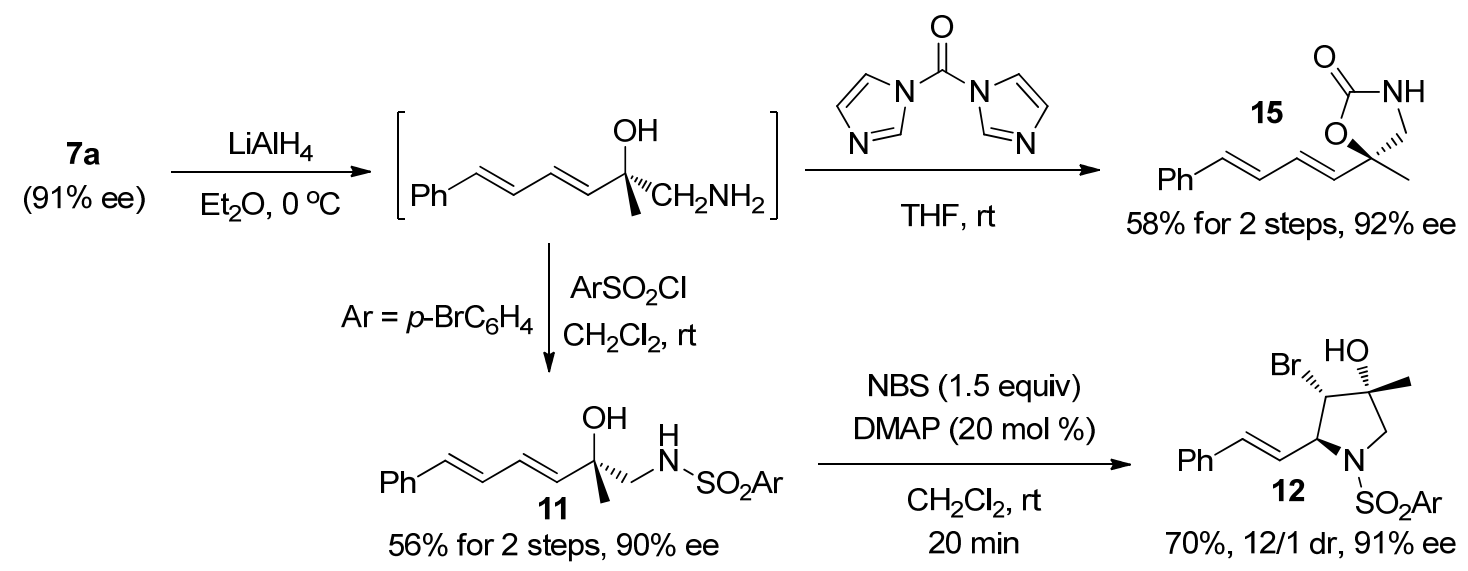<smiles>O=S(=O)(NCC(O)(I)/C=C/C=C/c1ccccc1)c1ccc(Br)cc1</smiles>

To a flamed dried three-neck flask were sequentially added $\mathrm{LiAlH}_{4}$ (76.0 mg, $2.0 \mathrm{mmol})$, anhydrous THF $(2.5 \mathrm{~mL})$ and $\mathrm{Et}_{2} \mathrm{O}(12.5 \mathrm{~mL})$. After the resulting solution was cooled down to $0{ }^{\circ} \mathrm{C}$ by an ice bath, a solution of $7 \mathbf{a}(135.5 \mathrm{mg}, 0.5 \mathrm{mmol})$ in $\mathrm{Et}_{2} \mathrm{O}(10 \mathrm{~mL})$ was added dropwise during a period of 10 mins. After the mixture was slowly warmed to room temperature (rt) and stirred for an additional hour, wet ether $(10 \mathrm{~mL})$ was slowly added, followed by the addition of $\mathrm{Na}_{2} \mathrm{SO}_{4}$. The mixture was stirred for $5 \mathrm{~min}$, and filtered. After removing the solvent under reduced pressure, the crude amino alcohol was dissolved in $2 \mathrm{~mL}$ anhydrous $\mathrm{CH}_{2} \mathrm{Cl}_{2}$, followed by the addition of $\mathrm{Et}_{3} \mathrm{~N}$ (0.2 mL, $\left.1.5 \mathrm{mmol}\right)$, 4-bromobenzene-1-sulfonyl chloride (254 mg, $1.0 \mathrm{mmol}$ ). The reaction was stirring at $\mathrm{rt}$ for about $16 \mathrm{~h}$, and then quenched with $\mathrm{NH}_{4} \mathrm{Cl}$ (sat.). After work-up procedure, column chromatograph could afford product 11 as a white solid (m.p. $123-124{ }^{\circ} \mathrm{C}$ ) in $56 \%$ yield for two steps, using PE/EtOAc (4:1) as the eluent; HPLC analysis (Chiralpak IC-H, ${ }^{i} \mathrm{PrOH} /$ hexane $=$ $15 / 85,1.0 \mathrm{~mL} / \mathrm{min}, 230 \mathrm{~nm} ; \mathrm{t}_{\mathrm{r}}$ (major) $=27.0 \mathrm{~min}, \mathrm{t}_{\mathrm{r}}$ (minor) $=30.13 \mathrm{~min}$ ) gave the isomeric composition of the product: $90 \%$ ee; $[\alpha]_{\mathrm{D}}{ }^{25}=+2.2\left(\mathrm{c}=1.0, \mathrm{CHCl}_{3}\right) ;{ }^{1} \mathrm{H}$ NMR $\left(400 \mathrm{MHz}\right.$, Acetone- $\left.d_{6}\right)$ : 7.83-7.76 (m, 4H), 7.46-7.44 (m, 2H), 7.34-7.30 (m, 2H), 7.22 (t, $J=7.3 \mathrm{~Hz}, 1 \mathrm{H}), 6.81$ (dd, $J=15.7$, $10.5 \mathrm{~Hz}, 1 \mathrm{H}), 6.59-6.44$ (m, 3H), 5.87 (d, $J=15.3 \mathrm{~Hz}, 1 \mathrm{H}), 4.00$ (s, 1H), 3.01-2.99 (m, 2H), 1.31 (s, $3 \mathrm{H}) ;{ }^{13} \mathrm{C}$ NMR (100 MHz, Acetone- $\left.d_{6}\right): \delta 141.33,139.90,138.34,133.09,132.90,129.93,129.82$, $129.65,129.52,128.31,127.23,127.17,72.41,53.90,26.17$; IR (ATR) v 3521.5, 3218.1, 1572.6, 1420.6, 1310.8, 1151.5, 1082.9, 996.3, 822.6, $741.7 \mathrm{~cm}^{-1}$; HRMS (ESI): Exact mass calcd for $\left(\mathrm{C}_{19} \mathrm{H}_{20}{ }^{79} \mathrm{BrNO}_{3} \mathrm{~S}+\mathrm{NH}_{4}{ }^{+}\right)$: 439.0686; found: 439.0671. The absolute configuration of $\mathbf{1 1}$ was determined to be $S$ by X-ray crystallographic analysis. 


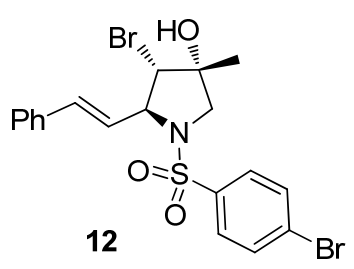

To a solution of $11(87.8 \mathrm{mg}, 0.20 \mathrm{mmol})$ in anhydrous $\mathrm{CH}_{2} \mathrm{Cl}_{2}(2.0 \mathrm{~mL})$ was added 4-(dimethylamino)-pyridin $\quad(2.5 \quad \mathrm{mg}, \quad 0.02 \quad \mathrm{mmol})$ and $\mathrm{N}$-Bromosuccinimide $(53.4 \mathrm{mg}, 0.30 \mathrm{mmol}$ ), and the resulting solution was stirred vigorously at $\mathrm{rt}$ till full conversion of $\mathbf{1 1}$ by TLC analysis. The distereoselectivity of $\mathbf{1 2}$ was determined to be $12: 1$ by ${ }^{1} \mathrm{H}$ NMR analysis of the crude reaction mixture. Column chromatography afforded 12 as a white solid (m.p. $185-186{ }^{\circ} \mathrm{C}$ ) in $70 \%$ yield; the relative configuration of $\mathbf{1 2}$ was determined by X-ray crystallographic analysis. HPLC analysis (Chiralpak AD-H, ${ }^{i} \mathrm{PrOH} /$ hexane $=10 / 90,1.0 \mathrm{~mL} / \mathrm{min}, 230 \mathrm{~nm} ; \mathrm{t}_{\mathrm{r}}$ (major) $=34.86 \mathrm{~min}, \mathrm{t}_{\mathrm{r}}$ $($ minor $)=25.70 \mathrm{~min})$ gave the isomeric composition of the product: $91 \% \mathrm{ee} ;[\alpha]_{\mathrm{D}}{ }^{25}=+150.8(\mathrm{c}=1.0$, $\left.\mathrm{CHCl}_{3}\right) ;{ }^{1} \mathrm{H}$ NMR $\left(400 \mathrm{MHz}, \mathrm{CDCl}_{3}\right)$ : 7.68-7.60 (m, 4H) 7.37-7.28 (m, 5H), $6.61(\mathrm{~d}, J=15.8 \mathrm{~Hz}, 1 \mathrm{H})$, $6.03(\mathrm{dd}, J=15.8,8.4 \mathrm{~Hz}, 1 \mathrm{H}), 4.12(\mathrm{t}, J=8.8 \mathrm{~Hz}, 1 \mathrm{H}), 3.90-3.86(\mathrm{~m}, 2 \mathrm{H}), 3.51(\mathrm{dd}, J=11.8,2.2 \mathrm{~Hz}$, $1 \mathrm{H}), 1.84(\mathrm{~s}, 1 \mathrm{H}), 1.33(\mathrm{~s}, 3 \mathrm{H}) ;{ }^{13} \mathrm{C} \mathrm{NMR}\left(100 \mathrm{MHz}, \mathrm{CDCl}_{3}\right): \delta 137.04,135.95,134.61,132.20$, $129.45,128.70,128.29,127.97,126.83,125.80,74.95,68.06,62.78,57.99,23.05$; IR (ATR) v 3486.1, 1575.1, 1451.4, 1342.4, 1159.8, 1029.4, 949.9, 825.3, 748.9, $696.2 \mathrm{~cm}^{-1}$; MS (EI): 499 (M $\left.\mathrm{M}^{+}, 1.37\right), 451$ (M+2, 2.84), 422 (12), 404 (39), 280 (76), 200 (62), 173 (87), 115 (69), 91 (100); HRMS (EI): Exact mass calcd for $\mathrm{C}_{19} \mathrm{H}_{19}{ }^{79} \mathrm{Br}_{2} \mathrm{NO}_{3} \mathrm{~S}$ : 498.9452 ; found: 498.9454 .

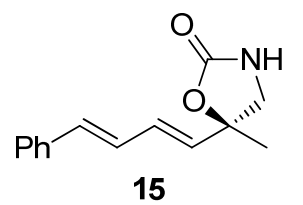

After the reduction of $7 \mathbf{a}(135.5 \mathrm{mg}, 0.5 \mathrm{mmol})$ by the above described procedure for 11, the crude amino alcohol was dissolved in anhydrous THF (2.0 $\mathrm{mL})$, followed by the addition of 1,1'-carbonyldiimidazole $(81.0 \mathrm{mg}, 0.5 \mathrm{mmol})$. The reaction mixture was vigorously stirred at $\mathrm{rt}$ till TLC analysis showed the completion of the reaction, and then removed the solvent under reduced pressure. The residue was directly subjected to column chromatograph to afford 15 as a white solid (m.p. 182-184 ${ }^{\circ} \mathrm{C}$ ) in $58 \%$ yield for two steps, using $\mathrm{CH}_{2} \mathrm{Cl}_{2} /$ EtOAc (4:1) as the eluent; HPLC analysis (Chiralpak AD-H, ${ }^{i} \mathrm{PrOH} /$ hexane $=20 / 80,1.0 \mathrm{~mL} / \mathrm{min}, 230 \mathrm{~nm} ; \mathrm{t}_{\mathrm{r}}($ major $)=8.62 \mathrm{~min}, \mathrm{t}_{\mathrm{r}}($ minor $\left.)=9.60 \mathrm{~min}\right)$ gave the isomeric composition of the product: $92 \%$ ee; $[\alpha]_{\mathrm{D}}{ }^{25}=+15.7\left(\mathrm{c}=1.0, \mathrm{CHCl}_{3}\right) ;{ }^{1} \mathrm{H} \mathrm{NMR}(400 \mathrm{MHz}$, $\left.\mathrm{CDCl}_{3}\right)$ : 7.41-7.39 (m, 2H), 7.34-7.30 (m, 2H), 7.24-7.22 (m, 1H), 6.78-6.72 (m, 1H), 6.64-6.60 (m, 1H), $6.51(\mathrm{dd}, J=15.3,10.3 \mathrm{~Hz}, 1 \mathrm{H}), 6.02(\mathrm{~s}, \mathrm{br}, 1 \mathrm{H}), 5.87$ (d, $J=15.3 \mathrm{~Hz}, 1 \mathrm{H}), 3.53$ (ABd, $J=8.4$ $\mathrm{Hz}, 1 \mathrm{H}), 3.44(\mathrm{ABd}, J=8.4 \mathrm{~Hz}, 1 \mathrm{H}), 1.62$ (s, 3H); ${ }^{13} \mathrm{C} \mathrm{NMR}\left(100 \mathrm{MHz}, \mathrm{CDCl}_{3}\right): \delta 159.34,136.85$, $134.57,133.72,130.23,128.73,128.01,127.36,126.59,81.99,52.33,25.78$; IR (ART) v 3320.2, 1763.6, 1725.2, 1241.8, 1076.0, 994.2, 960.7, 751.3, $690.8 \mathrm{~cm}^{-1}$; MS (EI): 229 (M+ 31), 184 (54), 170 (37), 141 (24), 128 (53), 115 (52), 91 (82), 77 (25); HRMS (EI): Exact mass calcd for $\mathrm{C}_{14} \mathrm{H}_{15} \mathrm{NO}_{2}$ : 229.1103; found: 229.1104 . 

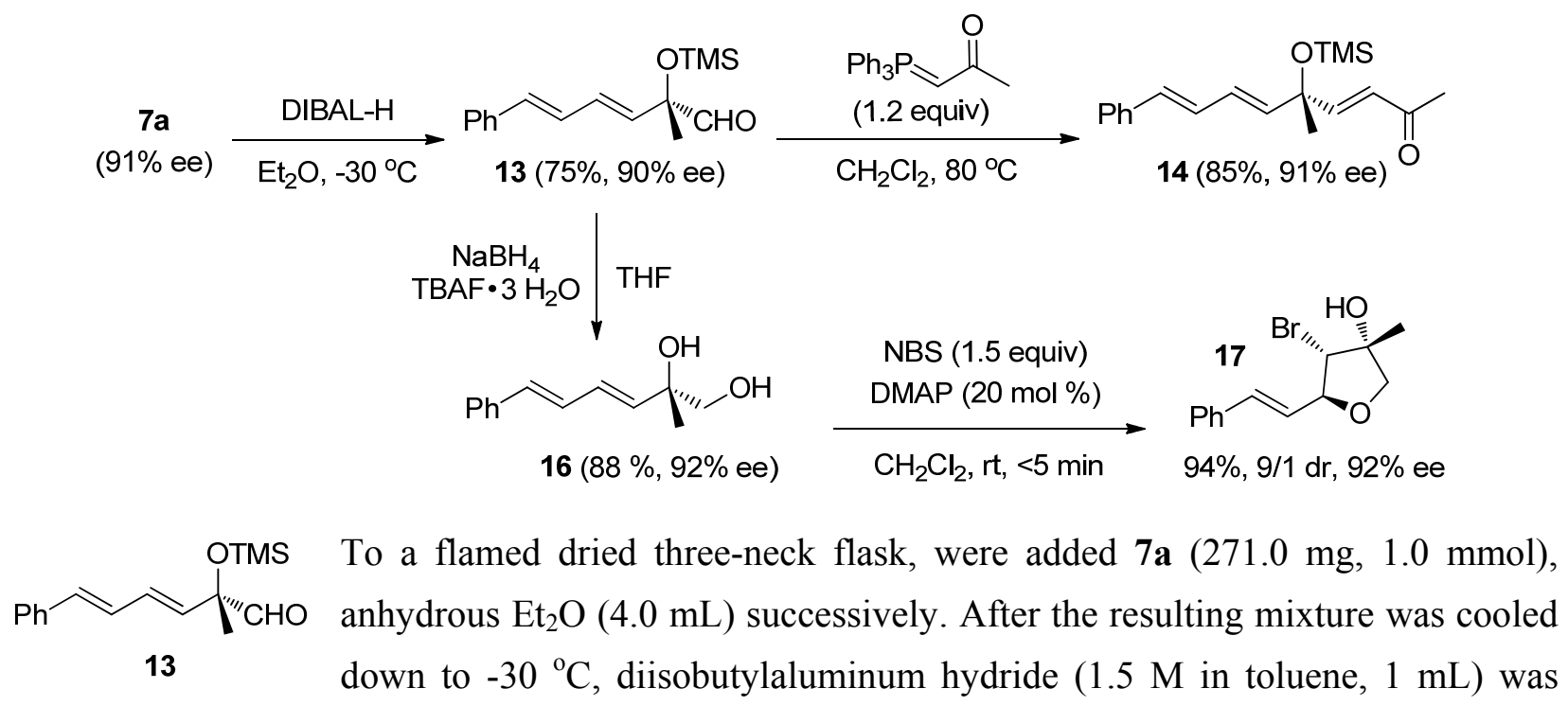
added dropwise during a period of 25 mins. After the full consumption of $7 \mathbf{a}, \mathrm{NH}_{4} \mathrm{Cl}$ (sat.) $(10 \mathrm{~mL})$ was slowly added to quench the reaction. After usual work-up procedure, column chromatograph afford 13 as slightly yellow oil in 75\% yield using $\mathrm{PE} / \mathrm{Et}_{2} \mathrm{O}$ as the eluent; HPLC analysis (Chiralcel $\mathrm{OJ}-\mathrm{H},{ }^{i} \mathrm{PrOH} / \mathrm{hexane}=1 / 99,1.0 \mathrm{~mL} / \mathrm{min}, 230 \mathrm{~nm}$; $\mathrm{t}_{\mathrm{r}}($ major $)=16.16 \mathrm{~min}, \mathrm{t}_{\mathrm{r}}($ minor $\left.)=13.09 \mathrm{~min}\right)$ gave the isomeric composition of the product: $90 \%$ ee; $[\alpha]_{\mathrm{D}}{ }^{25}=-225.3\left(\mathrm{c}=1.0, \mathrm{CHCl}_{3}\right) ;{ }^{1} \mathrm{H} \mathrm{NMR}$ $\left(400 \mathrm{MHz}, \mathrm{CDCl}_{3}\right): \delta 9.38(\mathrm{~s}, 1 \mathrm{H}), 7.41-7.39(\mathrm{~m}, 2 \mathrm{H}), 7.34-7.30(\mathrm{~m}, 2 \mathrm{H}), 7.26-7.22(\mathrm{~m}, 1 \mathrm{H})$, 6.80-6.74 (m, 1H), 6.64-6.60 (m, 1H), $6.50(\mathrm{dd}, J=15.2,10.5 \mathrm{~Hz}, 1 \mathrm{H}), 5.66(\mathrm{~d}, J=15.2 \mathrm{~Hz}, 1 \mathrm{H})$, $1.48(\mathrm{~s}, 3 \mathrm{H}), 0.20(\mathrm{~s}, 9 \mathrm{H}) ;{ }^{13} \mathrm{C}$ NMR $\left(100 \mathrm{MHz}, \mathrm{CDCl}_{3}\right): \delta 200.11,136.99,134.10,132.96,132.57$, 128.74, 127.95, 127.77, 126.58, 81.11, 22.95, 2.34; IR (ATR) v 3027.9, 2956.0, 2799.4, 1732.5, 1252.2, 1199.7, 990.8, 838.9, 749.1, $690.0 \mathrm{~cm}^{-1}$; MS (EI): $274\left(\mathrm{M}^{+}, 0.6\right), 259$ (1.8), 245 (96.7), 215 (4.3), 155 (27.3), 115 (11), 91 (4.3), 73 (100); HRMS (EI): Exact mass calcd for $\mathrm{C}_{16} \mathrm{H}_{22} \mathrm{O}_{2} \mathrm{Si}$ : 274.1389; found: 274.1390 .

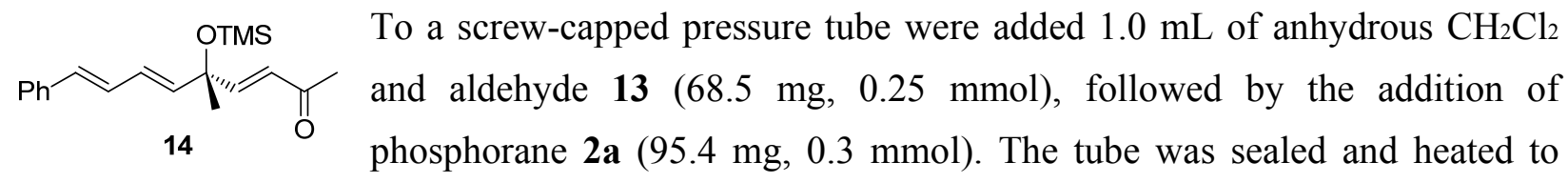
$80{ }^{\circ} \mathrm{C}$ until full consumption of $\mathbf{1 3}$ by TLC analysis. Direct column chromatograph afforded $\mathbf{1 4}$ as slightly yellow oil in $85 \%$ yield. HPLC analysis (Chiralpak AS-H, ${ }^{i} \mathrm{PrOH} / \mathrm{hexane}=1 / 99,1.0 \mathrm{~mL} / \mathrm{min}$, $230 \mathrm{~nm} ; \mathrm{t}_{\mathrm{r}}($ major $)=13.39 \mathrm{~min}, \mathrm{t}_{\mathrm{r}}($ minor $\left.)=11.55 \mathrm{~min}\right)$ gave the isomeric composition of the product: $91 \%$ ee, $[\alpha]_{\mathrm{D}}{ }^{25}=+33.5\left(\mathrm{c}=1.0, \mathrm{CHCl}_{3}\right) ;{ }^{1} \mathrm{H} \mathrm{NMR}\left(400 \mathrm{MHz}, \mathrm{CDCl}_{3}\right): 7.40-7.39(\mathrm{~m}, 2 \mathrm{H}), 7.34-7.32$ (m, 2H), 7.25-7.21 (m, 1H), 6.78-6.68 (m, 2H), 6.61-6.57 (m, 1H), 6.35 (dd, $J=15.3,10.4 \mathrm{~Hz}, 1 \mathrm{H})$, $6.24(\mathrm{~d}, J=15.8 \mathrm{~Hz}, 1 \mathrm{H}), 5.78(\mathrm{~d}, J=15.4 \mathrm{~Hz}, 1 \mathrm{H}), 2.28(\mathrm{~s}, 3 \mathrm{H}), 1.52(\mathrm{~s}, 3 \mathrm{H}), 0.16(\mathrm{~s}, 9 \mathrm{H}) ;{ }^{13} \mathrm{C} \mathrm{NMR}$ 
$\left(100 \mathrm{MHz}, \mathrm{CDCl}_{3}\right): \delta 199.09,152.32,137.86,137.09,133.53,129.74,128.73,128.07,127.83,127.43$, 126.50, 75.17, 27.47, 27.33, 2.53; IR (ART) v 3026.2, 2958.9, 1675.1, 1360.0, 1253.3, 1189.4, 1087.4, 987.3, 837.9, 750.0, $690.7 \mathrm{~cm}^{-1}$; MS (EI): 314 (M+ 4), 299 (6), 271 (74), 224 (17), 181 (38), 117 (23), 91 (35), 73 (100); HRMS (EI): Exact mass calcd for $\mathrm{C}_{19} \mathrm{H}_{26} \mathrm{O}_{2} \mathrm{Si}$ : 314.1702; found: 314.1700.<smiles>CC(O)(/C=C/C=C/c1ccccc1)CO</smiles>

16

To a solution of $13(82.2 \mathrm{mg}, 0.30 \mathrm{mmol})$ in $3.0 \mathrm{~mL}$ of anhydrous THF was sequentially added $\mathrm{NaBH}_{4}(22.8,0.60 \mathrm{mmol})$ and tetrabutylammonium fluoride trihydrate (189 mg, $0.6 \mathrm{mmol})$. The reaction mixture was stirred vigorously at $\mathrm{rt}$ till full conversion of 13, which was quenched by $\mathrm{NH}_{4} \mathrm{Cl}$ (sat.). After work-up, column chromatograph gave the diol 16 as white solid (m.p. 96-97 $\left.{ }^{\circ} \mathrm{C}\right)$ in $88 \%$ yield using PE/EtOAc (4:1) as the eluent; HPLC analysis (Chiralcel OJ-H, ${ }^{i} \mathrm{PrOH} / \mathrm{h} e x a n e=20 / 80,1.0 \mathrm{~mL} / \mathrm{min}, 230 \mathrm{~nm}$; $\mathrm{t}_{\mathrm{r}}$ (major) $=$ $11.13 \mathrm{~min}, \mathrm{t}_{\mathrm{r}}($ minor $\left.)=12.60 \mathrm{~min}\right)$ gave the isomeric composition of the product: $92 \%$ ee, $[\alpha]_{\mathrm{D}}{ }^{25}=$ $+2.6\left(\mathrm{c}=1.0, \mathrm{CHCl}_{3}\right) ;{ }^{1} \mathrm{H}$ NMR $\left(400 \mathrm{MHz}, \mathrm{CDCl}_{3}\right): 7.40-7.38(\mathrm{~m}, 2 \mathrm{H}), 7.33-7.29(\mathrm{~m}, 2 \mathrm{H}), 7.24-7.21$ $(\mathrm{m}, 1 \mathrm{H}), 6.77(\mathrm{dd}, J=15.5 \mathrm{~Hz}, 10.5 \mathrm{~Hz}, 1 \mathrm{H}), 6.60-6.48(\mathrm{~m}, 2 \mathrm{H}), 5.85(\mathrm{~d}, J=15.4 \mathrm{~Hz}, 1 \mathrm{H}), 3.56(\mathrm{ABd}$, $J=11.2 \mathrm{~Hz}, 1 \mathrm{H}), 3.49(\mathrm{ABd}, J=10.8 \mathrm{~Hz}, 1 \mathrm{H}), 2.29(\mathrm{~s}, \mathrm{br}, 1 \mathrm{H}), 2.01$ (s, br, $1 \mathrm{H}), 1.34(\mathrm{~s}, 3 \mathrm{H}) ;{ }^{13} \mathrm{C}$ NMR $\left(100 \mathrm{MHz}, \mathrm{CDCl}_{3}\right): \delta 137.27,137.18,133.03,130.09,128.70,128.19,127.71,126.46,73.47$, 70.04, 24.39; IR (ATR) v 3387.8, 3308.8, 2980.7, 1447.4, 1172.9, 1046.8, 996.5, 751.36, $692.3 \mathrm{~cm}^{-1}$; MS (EI): 204 (M+ , 9), 186 (27), 173 (54), 157 (59), 129 (55), 115 (37), 91 (100), 77 (20); HRMS (EI): Exact mass calcd for $\mathrm{C}_{13} \mathrm{H}_{16} \mathrm{O}_{2}: 204.1150$; found: 204.1147 .

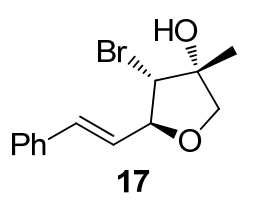

Compound 16 (51.0 $\mathrm{mg}, 0.25 \mathrm{mmol})$ was converted to 17 using the same procedure for the synthesis of $\mathbf{1 2}$. The distereoselectivity of $\mathbf{1 7}$ was determined to be $9: 1$ by ${ }^{1} \mathrm{H}$ NMR analysis of the crude reaction residue. Column chromatography afforded $\mathbf{1 7}$ as oily solid in 94\% yield as inseparable 13:1 (by ${ }^{1} \mathrm{H}-\mathrm{NMR}$ ) distereo-isomers; HPLC analysis $\left(\right.$ Chiralpak AS-H, ${ }^{i} \mathrm{PrOH} /$ hexane $=1 / 99,1.0 \mathrm{~mL} / \mathrm{min}, 230 \mathrm{~nm} ; \mathrm{t}_{\mathrm{r}}$ (major) $=37.63 \mathrm{~min}, \mathrm{t}_{\mathrm{r}}$ $($ minor $)=30.28 \mathrm{~min})$ gave the isomeric composition of the product: $92 \%$ ee, $[\alpha]_{\mathrm{D}}{ }^{25}=-64.8(\mathrm{c}=1.0$, $\mathrm{CHCl}_{3}$ ); ${ }^{1} \mathrm{H} \mathrm{NMR}\left(400 \mathrm{MHz}, \mathrm{CDCl}_{3}\right.$ ) (for the major distereo-isomer): 7.42-7.40 (m, 2H), 7.34-7.30 (m, 2H), 7.28-7.25 (m, 1H), $6.75(\mathrm{~d}, J=15.8 \mathrm{~Hz}, 1 \mathrm{H}), 6.17(\mathrm{dd}, J=15.8,7.1 \mathrm{~Hz}, 1 \mathrm{H}), 4.65-4.62$ (m, $1 \mathrm{H}), 4.10-4.07(\mathrm{~m}, 1 \mathrm{H}), 3.96-3.93(\mathrm{~m}, 1 \mathrm{H}), 3.83(\mathrm{~d}, J=9.7 \mathrm{~Hz}, 1 \mathrm{H}), 2.17(\mathrm{~s}, 1 \mathrm{H}), 1.39(\mathrm{~s}, 3 \mathrm{H}) ;{ }^{13} \mathrm{C}$ NMR (100 MHz, $\mathrm{CDCl}_{3}$ ) (for the major distereo-isomer): $\delta 136.26,134.16,128.67,128.15,126.80$, 125.51, 83.89, 76.98, 76.62, 62.60, 23.14; IR (ATR) v 3449.1, 2967.5, 2885.2, 1493.3, 1376.8, 1136.0, 1030.1, 965.2, 833.2, 751.2, $690.7 \mathrm{~cm}^{-1}$; MS (EI): $282\left(\mathrm{M}^{+}, 2.3\right), 282(\mathrm{M}+2,2.2), 203$ (22.6), 185 (13.9), 129 (100), 115 (19.0), 91 (35.4). HRMS (EI): Exact mass calcd for $\mathrm{C}_{13} \mathrm{H}_{15}{ }^{79} \mathrm{BrO}_{2}$ : 282.0255 ; found: 282.0256 . 


\section{Single-crystal X-ray crystallography of $11 \& 12$}

\section{(1) Single-Crystal X-ray Crystallography of 11}

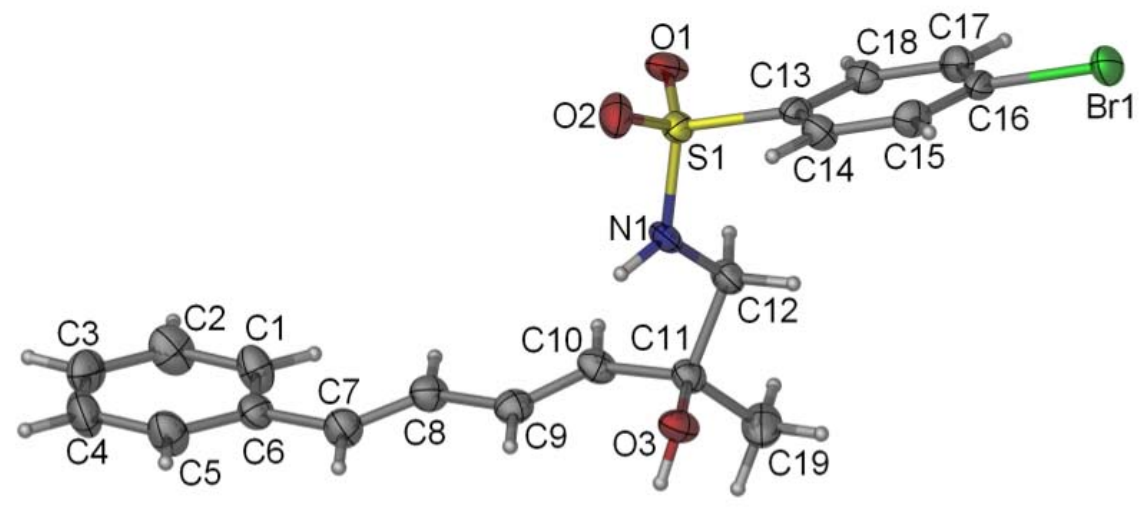

Data intensity of 11 was collected using a Bruker SMART APEX II (Mo radiation). The X-ray condition of was $50 \mathrm{kV} \times 30 \mathrm{~mA}$. Data collection and reduction were done by using the Bruker ApexII software package. The structure was solved by direct methods and refined by full-matrix least-squares on F2 with anisotropic displacement parameters for non-H atoms using SHELX-97. Hydrogen atoms were added at their geometrically idea positions and refined isotropically. Crystal data for 11: $\mathrm{C}_{19} \mathrm{H}_{20} \mathrm{BrNO}_{3} \mathrm{~S}, \mathrm{M}=422.33, \mathrm{~T}=173(2) \mathrm{K}, \lambda=0.71073 \AA$, Orthorhombic, space group P2(1)2(1)2(1), a $=5.9186(3) \AA, c=41.477(2) \AA, b=7.6659(4) \AA, V=1881.85(16) \AA \wedge 3, z=4, d$ calc $=1.491 \mathrm{Mg} / \mathrm{m}^{\wedge} 3$, 22569 reflections measured, 3484 unique [Rint $=0.0474], \mathrm{R} 1=0.0331, \mathrm{wR} 2=0.0721(I>2 \sigma)$, final $\mathrm{R} 1=0.0308, \mathrm{wR} 2=0.0711, \mathrm{GOF}=1.080$, and 226 parameters 
Table S8. Crystal data and structure refinement for 11.

\begin{tabular}{|ll|}
\hline Identification code & $\mathbf{1 1}$ \\
\hline Empirical formula & $\mathrm{C}_{19} \mathrm{H}_{20} \mathrm{BrNO}_{3} \mathrm{~S}$ \\
\hline Formula weight & 422.33 \\
\hline Temperature & $173(2) \mathrm{K}$ \\
\hline Wavelength & $0.71073 \AA$ \\
\hline Crystal system, space group & Orthorhombic, $\mathrm{P} 2(1) 2(1) 2(1)$ \\
\hline Unit cell dimensions & $\mathrm{a}=5.9186(3) \AA \quad$ alpha $=90 \mathrm{deg}$. \\
& $\mathrm{b}=7.6659(4) \AA \quad$ beta $=90 \mathrm{deg}$. \\
& $\mathrm{c}=41.477(2) \AA \quad$ gamma $=90$ deg. \\
\hline Volume & $1881.85(16) \AA \wedge 3$ \\
\hline Z, Calculated density & $4,1.491 \mathrm{Mg} / \mathrm{m}^{\wedge} 3$ \\
\hline Absorption coefficient & $2.313 \mathrm{~mm}{ }^{\wedge}-1$ \\
\hline F(000) & 864 \\
\hline Crystal size & $0.48 \times 0.24 \mathrm{x} 0.12$ mm \\
\hline Theta range for data collection & 2.70 to 25.46 deg. \\
\hline Limiting indices & $-7<=\mathrm{h}<=7,-9<=\mathrm{k}<=9,-42<=1<=50$ \\
\hline Reflections collected / unique & $22569 / 3484[\mathrm{R}($ int $)=0.0474]$ \\
\hline Completeness to theta $=25.46$ & $99.9 \%$ \\
\hline Absorption correction & Semi-empirical from equivalents \\
\hline Max. and min. transmission & 0.7688 and 0.4032 \\
\hline Refinement method & Full-matrix least-squares on $\mathrm{F}^{\wedge} 2$ \\
\hline Data / restraints / parameters & $3484 / 0 / 226$ \\
\hline Goodness-of-fit on $\mathrm{F}^{\wedge} 2$ & 1.080 \\
\hline Final R indices [I $>2$ sigma(I)] & $\mathrm{R} 1=0.0308, \mathrm{wR} 2=0.0711$ \\
\hline R indices (all data) & $\mathrm{R} 1=0.0331, \mathrm{wR} 2=0.0721$ \\
\hline Absolute structure parameter & $0.015(8)$ \\
\hline Largest diff. peak and hole & 0.401 and -0.492 e. $\AA^{\wedge}-3$ \\
\hline & \\
\hline
\end{tabular}

Table S9. Atomic coordinates $\left(\times 10^{\wedge} 4\right)$ and equivalent isotropic displacement parameters $\left(\AA^{\wedge} 2 \times 10^{\wedge} 3\right)$ for 11. U(eq) was defined as one third of the trace of the orthogonalized Uij tensor.

\begin{tabular}{lrrrr}
\hline & $\mathrm{x}$ & $\mathrm{y}$ & $\mathrm{z}$ & $\mathrm{U}(\mathrm{eq})$ \\
\hline $\mathrm{Br}(1)$ & $8080(1)$ & $4249(1)$ & $9590(1)$ & $36(1)$ \\
$\mathrm{S}(1)$ & $3573(1)$ & $5807(1)$ & $10965(1)$ & $24(1)$ \\
$\mathrm{O}(1)$ & $4606(4)$ & $4628(2)$ & $11188(1)$ & $34(1)$ \\
$\mathrm{O}(2)$ & $1187(3)$ & $5813(3)$ & $10926(1)$ & $36(1)$ \\
$\mathrm{O}(3)$ & $5240(4)$ & $11095(3)$ & $11075(1)$ & $31(1)$ \\
$\mathrm{N}(1)$ & $4261(4)$ & $7747(3)$ & $11076(1)$ & $25(1)$ \\
$\mathrm{C}(1)$ & $3094(6)$ & $10308(4)$ & $12811(1)$ & $37(1)$ \\
$\mathrm{C}(2)$ & $2426(6)$ & $10025(5)$ & $13124(1)$ & $43(1)$ \\
$\mathrm{C}(3)$ & $357(6)$ & $10613(5)$ & $13230(1)$ & $40(1)$ \\
$\mathrm{C}(4)$ & $-1017(6)$ & $11502(5)$ & $13026(1)$ & $42(1)$
\end{tabular}




\begin{tabular}{lrrrr}
\hline & & & & \\
$\mathrm{C}(5)$ & $-370(6)$ & $11790(5)$ & $12709(1)$ & $40(1)$ \\
$\mathrm{C}(6)$ & $1692(5)$ & $11198(4)$ & $12594(1)$ & $30(1)$ \\
$\mathrm{C}(7)$ & $2295(5)$ & $11437(4)$ & $12254(1)$ & $33(1)$ \\
$\mathrm{C}(8)$ & $3982(5)$ & $10646(4)$ & $12099(1)$ & $31(1)$ \\
$\mathrm{C}(9)$ & $4476(5)$ & $10833(4)$ & $11759(1)$ & $29(1)$ \\
$\mathrm{C}(10)$ & $6104(5)$ & $9941(4)$ & $11610(1)$ & $28(1)$ \\
$\mathrm{C}(11)$ & $6760(5)$ & $10054(4)$ & $11262(1)$ & $27(1)$ \\
$\mathrm{C}(12)$ & $6631(5)$ & $8253(4)$ & $11099(1)$ & $27(1)$ \\
$\mathrm{C}(13)$ & $4810(5)$ & $5389(3)$ & $10585(1)$ & $22(1)$ \\
$\mathrm{C}(14)$ & $3683(5)$ & $5876(4)$ & $10305(1)$ & $28(1)$ \\
$\mathrm{C}(15)$ & $4642(5)$ & $5535(4)$ & $10008(1)$ & $29(1)$ \\
$\mathrm{C}(16)$ & $6720(5)$ & $4733(3)$ & $9995(1)$ & $26(1)$ \\
$\mathrm{C}(17)$ & $7862(5)$ & $4250(4)$ & $10271(1)$ & $29(1)$ \\
$\mathrm{C}(18)$ & $6904(5)$ & $4585(3)$ & $10569(1)$ & $26(1)$ \\
$\mathrm{C}(19)$ & $9162(5)$ & $10732(5)$ & $11234(1)$ & $43(1)$ \\
& & & & \\
\hline
\end{tabular}

Table S10. Bond lengths $[\AA]$ and angles [deg] for 11 .

\begin{tabular}{lc}
\hline $\mathrm{Br}(1)-\mathrm{C}(16)$ & $1.898(3)$ \\
$\mathrm{S}(1)-\mathrm{O}(2)$ & $1.422(2)$ \\
$\mathrm{S}(1)-\mathrm{O}(1)$ & $1.428(2)$ \\
$\mathrm{S}(1)-\mathrm{N}(1)$ & $1.609(2)$ \\
$\mathrm{S}(1)-\mathrm{C}(13)$ & $1.769(3)$ \\
$\mathrm{O}(3)-\mathrm{C}(11)$ & $1.430(4)$ \\
$\mathrm{O}(3)-\mathrm{H}(3 \mathrm{~B})$ & 0.8400 \\
$\mathrm{~N}(1)-\mathrm{C}(12)$ & $1.459(4)$ \\
$\mathrm{N}(1)-\mathrm{H}(1 \mathrm{~B})$ & 0.8800 \\
$\mathrm{C}(1)-\mathrm{C}(2)$ & $1.374(5)$ \\
$\mathrm{C}(1)-\mathrm{C}(6)$ & $1.402(4)$ \\
$\mathrm{C}(1)-\mathrm{H}(1 \mathrm{~A})$ & 0.9500 \\
$\mathrm{C}(2)-\mathrm{C}(3)$ & $1.378(5)$ \\
$\mathrm{C}(2)-\mathrm{H}(2 \mathrm{~A})$ & 0.9500 \\
$\mathrm{C}(3)-\mathrm{C}(4)$ & $1.359(5)$ \\
$\mathrm{C}(3)-\mathrm{H}(3 \mathrm{~A})$ & 0.9500 \\
$\mathrm{C}(4)-\mathrm{C}(5)$ & $1.385(5)$ \\
$\mathrm{C}(4)-\mathrm{H}(4 \mathrm{~A})$ & 0.9500 \\
$\mathrm{C}(5)-\mathrm{C}(6)$ & $1.387(5)$ \\
$\mathrm{C}(5)-\mathrm{H}(5 \mathrm{~A})$ & 0.9500 \\
$\mathrm{C}(6)-\mathrm{C}(7)$ & $1.465(4)$ \\
$\mathrm{C}(7)-\mathrm{C}(8)$ & $1.334(4)$ \\
$\mathrm{C}(7)-\mathrm{H}(7 \mathrm{~A})$ & 0.9500 \\
$\mathrm{C}(8)-\mathrm{C}(9)$ & $1.446(4)$ \\
$\mathrm{C}(8)-\mathrm{H}(8 \mathrm{~A})$ & 0.9500 \\
$\mathrm{C}(9)-\mathrm{C}(10)$ & $1.333(4)$ \\
$\mathrm{C}(9)-\mathrm{H}(9 \mathrm{~A})$ & 0.9500 \\
$\mathrm{C}(10)-\mathrm{C}(11)$ & $1.498(4)$ \\
&
\end{tabular}




\begin{tabular}{|c|c|}
\hline $\mathrm{C}(10)-\mathrm{H}(10 \mathrm{~A})$ & 0.9500 \\
\hline$C(11)-C(19)$ & $1.518(4)$ \\
\hline $\mathrm{C}(11)-\mathrm{C}(12)$ & $1.539(4)$ \\
\hline $\mathrm{C}(12)-\mathrm{H}(12 \mathrm{~A})$ & 0.9900 \\
\hline $\mathrm{C}(12)-\mathrm{H}(12 \mathrm{~B})$ & 0.9900 \\
\hline $\mathrm{C}(13)-\mathrm{C}(18)$ & $1.385(4)$ \\
\hline$C(13)-C(14)$ & $1.389(4)$ \\
\hline$C(14)-C(15)$ & $1.382(4)$ \\
\hline $\mathrm{C}(14)-\mathrm{H}(14 \mathrm{~A})$ & 0.9500 \\
\hline $\mathrm{C}(15)-\mathrm{C}(16)$ & $1.376(4)$ \\
\hline $\mathrm{C}(15)-\mathrm{H}(15 \mathrm{~A})$ & 0.9500 \\
\hline $\mathrm{C}(16)-\mathrm{C}(17)$ & $1.379(4)$ \\
\hline $\mathrm{C}(17)-\mathrm{C}(18)$ & $1.386(4)$ \\
\hline $\mathrm{C}(17)-\mathrm{H}(17 \mathrm{~A})$ & 0.9500 \\
\hline $\mathrm{C}(18)-\mathrm{H}(18 \mathrm{~A})$ & 0.9500 \\
\hline $\mathrm{C}(19)-\mathrm{H}(19 \mathrm{~A})$ & 0.9800 \\
\hline $\mathrm{C}(19)-\mathrm{H}(19 \mathrm{~B})$ & 0.9800 \\
\hline C(19)-H(19C) & 0.9800 \\
\hline $\mathrm{O}(2)-\mathrm{S}(1)-\mathrm{O}(1)$ & $120.11(15)$ \\
\hline $\mathrm{O}(2)-\mathrm{S}(1)-\mathrm{N}(1)$ & $106.33(14)$ \\
\hline $\mathrm{O}(1)-\mathrm{S}(1)-\mathrm{N}(1)$ & $107.03(14)$ \\
\hline $\mathrm{O}(2)-\mathrm{S}(1)-\mathrm{C}(13)$ & $107.99(13)$ \\
\hline $\mathrm{O}(1)-\mathrm{S}(1)-\mathrm{C}(13)$ & $106.49(13)$ \\
\hline $\mathrm{N}(1)-\mathrm{S}(1)-\mathrm{C}(13)$ & $108.47(13)$ \\
\hline $\mathrm{C}(11)-\mathrm{O}(3)-\mathrm{H}(3 \mathrm{~B})$ & 109.5 \\
\hline $\mathrm{C}(12)-\mathrm{N}(1)-\mathrm{S}(1)$ & $120.53(19)$ \\
\hline $\mathrm{C}(12)-\mathrm{N}(1)-\mathrm{H}(1 \mathrm{~B})$ & 119.7 \\
\hline $\mathrm{S}(1)-\mathrm{N}(1)-\mathrm{H}(1 \mathrm{~B})$ & 119.7 \\
\hline$C(2)-C(1)-C(6)$ & $120.9(3)$ \\
\hline $\mathrm{C}(2)-\mathrm{C}(1)-\mathrm{H}(1 \mathrm{~A})$ & 119.6 \\
\hline $\mathrm{C}(6)-\mathrm{C}(1)-\mathrm{H}(1 \mathrm{~A})$ & 119.6 \\
\hline$C(1)-C(2)-C(3)$ & $120.5(3)$ \\
\hline $\mathrm{C}(1)-\mathrm{C}(2)-\mathrm{H}(2 \mathrm{~A})$ & 119.8 \\
\hline $\mathrm{C}(3)-\mathrm{C}(2)-\mathrm{H}(2 \mathrm{~A})$ & 119.8 \\
\hline$C(4)-C(3)-C(2)$ & $119.7(3)$ \\
\hline $\mathrm{C}(4)-\mathrm{C}(3)-\mathrm{H}(3 \mathrm{~A})$ & 120.2 \\
\hline $\mathrm{C}(2)-\mathrm{C}(3)-\mathrm{H}(3 \mathrm{~A})$ & 120.2 \\
\hline$C(3)-C(4)-C(5)$ & $120.5(3)$ \\
\hline $\mathrm{C}(3)-\mathrm{C}(4)-\mathrm{H}(4 \mathrm{~A})$ & 119.8 \\
\hline $\mathrm{C}(5)-\mathrm{C}(4)-\mathrm{H}(4 \mathrm{~A})$ & 119.8 \\
\hline$C(4)-C(5)-C(6)$ & $121.2(3)$ \\
\hline $\mathrm{C}(4)-\mathrm{C}(5)-\mathrm{H}(5 \mathrm{~A})$ & 119.4 \\
\hline $\mathrm{C}(6)-\mathrm{C}(5)-\mathrm{H}(5 \mathrm{~A})$ & 119.4 \\
\hline$C(5)-C(6)-C(1)$ & $117.3(3)$ \\
\hline$C(5)-C(6)-C(7)$ & $120.4(3)$ \\
\hline$C(1)-C(6)-C(7)$ & $122.3(3)$ \\
\hline
\end{tabular}




\begin{tabular}{|c|c|}
\hline$C(8)-C(7)-C(6)$ & $126.1(3)$ \\
\hline $\mathrm{C}(8)-\mathrm{C}(7)-\mathrm{H}(7 \mathrm{~A})$ & 116.9 \\
\hline $\mathrm{C}(6)-\mathrm{C}(7)-\mathrm{H}(7 \mathrm{~A})$ & 116.9 \\
\hline$C(7)-C(8)-C(9)$ & $125.2(3)$ \\
\hline $\mathrm{C}(7)-\mathrm{C}(8)-\mathrm{H}(8 \mathrm{~A})$ & 117.4 \\
\hline $\mathrm{C}(9)-\mathrm{C}(8)-\mathrm{H}(8 \mathrm{~A})$ & 117.4 \\
\hline $\mathrm{C}(10)-\mathrm{C}(9)-\mathrm{C}(8)$ & $123.1(3)$ \\
\hline $\mathrm{C}(10)-\mathrm{C}(9)-\mathrm{H}(9 \mathrm{~A})$ & 118.4 \\
\hline $\mathrm{C}(8)-\mathrm{C}(9)-\mathrm{H}(9 \mathrm{~A})$ & 118.4 \\
\hline$C(9)-C(10)-C(11)$ & $127.2(3)$ \\
\hline $\mathrm{C}(9)-\mathrm{C}(10)-\mathrm{H}(10 \mathrm{~A})$ & 116.4 \\
\hline $\mathrm{C}(11)-\mathrm{C}(10)-\mathrm{H}(10 \mathrm{~A})$ & 116.4 \\
\hline $\mathrm{O}(3)-\mathrm{C}(11)-\mathrm{C}(10)$ & $113.1(2)$ \\
\hline $\mathrm{O}(3)-\mathrm{C}(11)-\mathrm{C}(19)$ & $110.9(3)$ \\
\hline $\mathrm{C}(10)-\mathrm{C}(11)-\mathrm{C}(19)$ & $109.7(3)$ \\
\hline $\mathrm{O}(3)-\mathrm{C}(11)-\mathrm{C}(12)$ & $103.4(2)$ \\
\hline $\mathrm{C}(10)-\mathrm{C}(11)-\mathrm{C}(12)$ & $111.0(2)$ \\
\hline$C(19)-C(11)-C(12)$ & $108.6(3)$ \\
\hline $\mathrm{N}(1)-\mathrm{C}(12)-\mathrm{C}(11)$ & $108.4(2)$ \\
\hline $\mathrm{N}(1)-\mathrm{C}(12)-\mathrm{H}(12 \mathrm{~A})$ & 110.0 \\
\hline $\mathrm{C}(11)-\mathrm{C}(12)-\mathrm{H}(12 \mathrm{~A})$ & 110.0 \\
\hline $\mathrm{N}(1)-\mathrm{C}(12)-\mathrm{H}(12 \mathrm{~B})$ & 110.0 \\
\hline $\mathrm{C}(11)-\mathrm{C}(12)-\mathrm{H}(12 \mathrm{~B})$ & 110.0 \\
\hline $\mathrm{H}(12 \mathrm{~A})-\mathrm{C}(12)-\mathrm{H}(12 \mathrm{~B})$ & 108.4 \\
\hline$C(18)-C(13)-C(14)$ & $120.7(3)$ \\
\hline $\mathrm{C}(18)-\mathrm{C}(13)-\mathrm{S}(1)$ & $119.5(2)$ \\
\hline $\mathrm{C}(14)-\mathrm{C}(13)-\mathrm{S}(1)$ & $119.8(2)$ \\
\hline $\mathrm{C}(15)-\mathrm{C}(14)-\mathrm{C}(13)$ & $119.8(3)$ \\
\hline $\mathrm{C}(15)-\mathrm{C}(14)-\mathrm{H}(14 \mathrm{~A})$ & 120.1 \\
\hline $\mathrm{C}(13)-\mathrm{C}(14)-\mathrm{H}(14 \mathrm{~A})$ & 120.1 \\
\hline$C(16)-C(15)-C(14)$ & $119.2(3)$ \\
\hline $\mathrm{C}(16)-\mathrm{C}(15)-\mathrm{H}(15 \mathrm{~A})$ & 120.4 \\
\hline $\mathrm{C}(14)-\mathrm{C}(15)-\mathrm{H}(15 \mathrm{~A})$ & 120.4 \\
\hline$C(15)-C(16)-C(17)$ & $121.6(3)$ \\
\hline $\mathrm{C}(15)-\mathrm{C}(16)-\mathrm{Br}(1)$ & $120.1(2)$ \\
\hline $\mathrm{C}(17)-\mathrm{C}(16)-\mathrm{Br}(1)$ & $118.2(2)$ \\
\hline $\mathrm{C}(16)-\mathrm{C}(17)-\mathrm{C}(18)$ & $119.4(3)$ \\
\hline $\mathrm{C}(16)-\mathrm{C}(17)-\mathrm{H}(17 \mathrm{~A})$ & 120.3 \\
\hline $\mathrm{C}(18)-\mathrm{C}(17)-\mathrm{H}(17 \mathrm{~A})$ & 120.3 \\
\hline $\mathrm{C}(13)-\mathrm{C}(18)-\mathrm{C}(17)$ & $119.4(3)$ \\
\hline $\mathrm{C}(13)-\mathrm{C}(18)-\mathrm{H}(18 \mathrm{~A})$ & 120.3 \\
\hline $\mathrm{C}(17)-\mathrm{C}(18)-\mathrm{H}(18 \mathrm{~A})$ & 120.3 \\
\hline $\mathrm{C}(11)-\mathrm{C}(19)-\mathrm{H}(19 \mathrm{~A})$ & 109.5 \\
\hline $\mathrm{C}(11)-\mathrm{C}(19)-\mathrm{H}(19 \mathrm{~B})$ & 109.5 \\
\hline $\mathrm{H}(19 \mathrm{~A})-\mathrm{C}(19)-\mathrm{H}(19 \mathrm{~B})$ & 109.5 \\
\hline $\mathrm{C}(11)-\mathrm{C}(19)-\mathrm{H}(19 \mathrm{C})$ & 109.5 \\
\hline $\mathrm{H}(19 \mathrm{~A})-\mathrm{C}(19)-\mathrm{H}(19 \mathrm{C})$ & 109.5 \\
\hline
\end{tabular}


H(19B)-C(19)-H(19C) $\quad 109.5$

Symmetry transformations used to generate equivalent atoms:

Table S11. Anisotropic displacement parameters $\left(\AA^{\wedge} 2 \times 10^{\wedge} 3\right)$ for 11 . The anisotropic displacement factor exponent takes the form: $-2 \mathrm{pi}^{\wedge} 2\left[\mathrm{~h}^{\wedge} 2 \mathrm{a}^{* \wedge} 2 \mathrm{U} 11+\ldots+2 \mathrm{hk} \mathrm{a}^{*} \mathrm{~b}^{*} \mathrm{U} 12\right]$

\begin{tabular}{lcccrrr}
\hline & $\mathrm{U} 11$ & $\mathrm{U} 22$ & $\mathrm{U} 33$ & $\mathrm{U} 23$ & $\mathrm{U} 13$ & $\mathrm{U} 12$ \\
\hline $\mathrm{Br}(1)$ & $50(1)$ & $33(1)$ & $26(1)$ & $-2(1)$ & $12(1)$ & $2(1)$ \\
$\mathrm{S}(1)$ & $30(1)$ & $22(1)$ & $20(1)$ & $-3(1)$ & $1(1)$ & $-3(1)$ \\
$\mathrm{O}(1)$ & $57(1)$ & $18(1)$ & $26(1)$ & $-1(1)$ & $0(1)$ & $2(1)$ \\
$\mathrm{O}(2)$ & $30(1)$ & $46(1)$ & $34(1)$ & $-11(1)$ & $7(1)$ & $-9(1)$ \\
$\mathrm{O}(3)$ & $45(1)$ & $22(1)$ & $24(1)$ & $-1(1)$ & $-2(1)$ & $1(1)$ \\
$\mathrm{N}(1)$ & $25(1)$ & $20(1)$ & $31(2)$ & $-8(1)$ & $2(1)$ & $4(1)$ \\
$\mathrm{C}(1)$ & $35(2)$ & $48(2)$ & $28(2)$ & $-7(1)$ & $-2(1)$ & $8(2)$ \\
$\mathrm{C}(2)$ & $50(2)$ & $51(2)$ & $27(2)$ & $-1(2)$ & $-10(2)$ & $10(2)$ \\
$\mathrm{C}(3)$ & $52(2)$ & $42(2)$ & $24(2)$ & $-1(2)$ & $5(1)$ & $-4(2)$ \\
$\mathrm{C}(4)$ & $41(2)$ & $46(2)$ & $39(2)$ & $-10(2)$ & $11(2)$ & $5(2)$ \\
$\mathrm{C}(5)$ & $46(2)$ & $40(2)$ & $33(2)$ & $2(2)$ & $0(2)$ & $13(2)$ \\
$\mathrm{C}(6)$ & $35(2)$ & $28(2)$ & $26(2)$ & $-4(1)$ & $1(1)$ & $-1(1)$ \\
$\mathrm{C}(7)$ & $36(2)$ & $33(2)$ & $30(2)$ & $2(1)$ & $-1(1)$ & $4(1)$ \\
$\mathrm{C}(8)$ & $38(2)$ & $28(2)$ & $26(2)$ & $0(1)$ & $-2(1)$ & $0(1)$ \\
$\mathrm{C}(9)$ & $32(1)$ & $27(2)$ & $28(2)$ & $-1(1)$ & $-4(1)$ & $-4(1)$ \\
$\mathrm{C}(10)$ & $33(2)$ & $26(1)$ & $24(2)$ & $-5(1)$ & $-6(1)$ & $1(1)$ \\
$\mathrm{C}(11)$ & $31(2)$ & $25(1)$ & $24(2)$ & $-3(1)$ & $0(1)$ & $1(1)$ \\
$\mathrm{C}(12)$ & $27(2)$ & $25(1)$ & $29(2)$ & $-5(1)$ & $1(1)$ & $2(1)$ \\
$\mathrm{C}(13)$ & $30(1)$ & $18(1)$ & $20(2)$ & $-3(1)$ & $0(1)$ & $-4(1)$ \\
$\mathrm{C}(14)$ & $30(1)$ & $26(1)$ & $26(2)$ & $-1(1)$ & $-1(1)$ & $4(1)$ \\
$\mathrm{C}(15)$ & $36(2)$ & $30(2)$ & $21(2)$ & $4(1)$ & $-4(1)$ & $1(1)$ \\
$\mathrm{C}(16)$ & $36(2)$ & $20(1)$ & $22(2)$ & $-3(1)$ & $8(1)$ & $-6(1)$ \\
$\mathrm{C}(17)$ & $24(1)$ & $29(1)$ & $33(2)$ & $0(1)$ & $2(1)$ & $1(2)$ \\
$\mathrm{C}(18)$ & $25(1)$ & $25(1)$ & $28(2)$ & $-3(1)$ & $-5(1)$ & $-2(1)$ \\
$\mathrm{C}(19)$ & $40(2)$ & $46(2)$ & $44(2)$ & $-16(2)$ & $6(2)$ & $-15(2)$ \\
& & & & & & \\
\hline & & & & & &
\end{tabular}

Table S12. Hydrogen coordinates $\left(\mathrm{x} 10^{\wedge} 4\right)$ and isotropic displacement parameters $\left(\mathrm{A}^{\wedge} 2 \times 10^{\wedge} 3\right)$ for 11.

\begin{tabular}{lcrcc}
\hline & $x$ & $y$ & $z$ & $U($ eq $)$ \\
\hline $\mathrm{H}(3 \mathrm{~B})$ & 5269 & 12128 & 11143 & 46 \\
$\mathrm{H}(1 \mathrm{~B})$ & 3198 & 8510 & 11122 & 30 \\
$\mathrm{H}(1 \mathrm{~A})$ & 4525 & 9894 & 12741 & 44 \\
$\mathrm{H}(2 \mathrm{~A})$ & 3398 & 9420 & 13268 & 52 \\
$\mathrm{H}(3 \mathrm{~A})$ & -108 & 10398 & 13446 & 47 \\
& & & &
\end{tabular}




\begin{tabular}{lrrrl}
\hline & & & & \\
$\mathrm{H}(4 \mathrm{~A})$ & -2431 & 11929 & 13100 & 50 \\
$\mathrm{H}(5 \mathrm{~A})$ & -1356 & 12403 & 12568 & 48 \\
$\mathrm{H}(7 \mathrm{~A})$ & 1400 & 12230 & 12133 & 39 \\
$\mathrm{H}(8 \mathrm{~A})$ & 4936 & 9901 & 12221 & 37 \\
$\mathrm{H}(9 \mathrm{~A})$ & 3598 & 11629 & 11636 & 35 \\
$\mathrm{H}(10 \mathrm{~A})$ & 6941 & 9146 & 11739 & 33 \\
$\mathrm{H}(12 \mathrm{~A})$ & 7313 & 8307 & 10882 & 32 \\
$\mathrm{H}(12 \mathrm{~B})$ & 7475 & 7383 & 11228 & 32 \\
$\mathrm{H}(14 \mathrm{~A})$ & 2258 & 6441 & 10318 & 33 \\
$\mathrm{H}(15 \mathrm{~A})$ & 3876 & 5849 & 9815 & 35 \\
$\mathrm{H}(17 \mathrm{~A})$ & 9292 & 3693 & 10256 & 34 \\
$\mathrm{H}(18 \mathrm{~A})$ & 7675 & 4267 & 10761 & 31 \\
$\mathrm{H}(19 \mathrm{~A})$ & 9271 & 11876 & 11338 & 65 \\
$\mathrm{H}(19 \mathrm{~B})$ & 9567 & 10842 & 11006 & 65 \\
$\mathrm{H}(19 \mathrm{C})$ & 10199 & 9915 & 11339 & 65 \\
& & & & \\
\hline
\end{tabular}

Table S13. Torsion angles [deg] for 11.

\begin{tabular}{l}
\hline $\mathrm{O}(2)-\mathrm{S}(1)-\mathrm{N}(1)-\mathrm{C}(12)$ \\
$\mathrm{O}(1)-\mathrm{S}(1)-\mathrm{N}(1)-\mathrm{C}(12)$ \\
$\mathrm{C}(13)-\mathrm{S}(1)-\mathrm{N}(1)-\mathrm{C}(12)$ \\
$\mathrm{C}(6)-\mathrm{C}(1)-\mathrm{C}(2)-\mathrm{C}(3)$ \\
$\mathrm{C}(1)-\mathrm{C}(2)-\mathrm{C}(3)-\mathrm{C}(4)$ \\
$\mathrm{C}(2)-\mathrm{C}(3)-\mathrm{C}(4)-\mathrm{C}(5)$ \\
$\mathrm{C}(3)-\mathrm{C}(4)-\mathrm{C}(5)-\mathrm{C}(6)$ \\
$\mathrm{C}(4)-\mathrm{C}(5)-\mathrm{C}(6)-\mathrm{C}(1)$ \\
$\mathrm{C}(4)-\mathrm{C}(5)-\mathrm{C}(6)-\mathrm{C}(7)$ \\
$\mathrm{C}(2)-\mathrm{C}(1)-\mathrm{C}(6)-\mathrm{C}(5)$ \\
$\mathrm{C}(2)-\mathrm{C}(1)-\mathrm{C}(6)-\mathrm{C}(7)$ \\
$\mathrm{C}(5)-\mathrm{C}(6)-\mathrm{C}(7)-\mathrm{C}(8)$ \\
$\mathrm{C}(1)-\mathrm{C}(6)-\mathrm{C}(7)-\mathrm{C}(8)$ \\
$\mathrm{C}(6)-\mathrm{C}(7)-\mathrm{C}(8)-\mathrm{C}(9)$ \\
$\mathrm{C}(7)-\mathrm{C}(8)-\mathrm{C}(9)-\mathrm{C}(10)$ \\
$\mathrm{C}(8)-\mathrm{C}(9)-\mathrm{C}(10)-\mathrm{C}(11)$ \\
$\mathrm{C}(9)-\mathrm{C}(10)-\mathrm{C}(11)-\mathrm{O}(3)$ \\
$\mathrm{C}(9)-\mathrm{C}(10)-\mathrm{C}(11)-\mathrm{C}(19)$ \\
$\mathrm{C}(9)-\mathrm{C}(10)-\mathrm{C}(11)-\mathrm{C}(12)$ \\
$\mathrm{S}(1)-\mathrm{N}(1)-\mathrm{C}(12)-\mathrm{C}(11)$ \\
$\mathrm{O}(3)-\mathrm{C}(11)-\mathrm{C}(12)-\mathrm{N}(1)$ \\
$\mathrm{C}(10)-\mathrm{C}(11)-\mathrm{C}(12)-\mathrm{N}(1)$ \\
$\mathrm{C}(19)-\mathrm{C}(11)-\mathrm{C}(12)-\mathrm{N}(1)$ \\
$\mathrm{O}(2)-\mathrm{S}(1)-\mathrm{C}(13)-\mathrm{C}(18)$ \\
$\mathrm{O}(1)-\mathrm{S}(1)-\mathrm{C}(13)-\mathrm{C}(18)$ \\
$\mathrm{N}(1)-\mathrm{S}(1)-\mathrm{C}(13)-\mathrm{C}(18)$ \\
$\mathrm{O}(2)-\mathrm{S}(1)-\mathrm{C}(13)-\mathrm{C}(14)$
\end{tabular}

$$
\begin{array}{r}
-172.4(2) \\
58.1(3) \\
-56.5(3) \\
0.1(6) \\
-1.0(6) \\
1.3(6) \\
-0.6(6) \\
-0.3(5) \\
176.8(3) \\
0.6(5) \\
-176.5(3) \\
-165.9(3) \\
11.2(5) \\
176.6(3) \\
-176.1(3) \\
-179.3(3) \\
-7.9(4) \\
116.4(4) \\
-123.5(3) \\
-170.8(2) \\
-52.2(3) \\
69.3(3) \\
-170.0(3) \\
-152.2(2) \\
-22.0(3) \\
92.9(2) \\
28.1(3)
\end{array}
$$


$\mathrm{O}(1)-\mathrm{S}(1)-\mathrm{C}(13)-\mathrm{C}(14)$

$\mathrm{N}(1)-\mathrm{S}(1)-\mathrm{C}(13)-\mathrm{C}(14)$

$\mathrm{C}(18)-\mathrm{C}(13)-\mathrm{C}(14)-\mathrm{C}(15)$

$\mathrm{S}(1)-\mathrm{C}(13)-\mathrm{C}(14)-\mathrm{C}(15)$

C(13)-C(14)-C(15)-C(16)

C(14)-C(15)-C(16)-C(17)

$\mathrm{C}(14)-\mathrm{C}(15)-\mathrm{C}(16)-\mathrm{Br}(1)$

$\mathrm{C}(15)-\mathrm{C}(16)-\mathrm{C}(17)-\mathrm{C}(18)$

$\mathrm{Br}(1)-\mathrm{C}(16)-\mathrm{C}(17)-\mathrm{C}(18)$

C(14)-C(13)-C(18)-C(17)

$\mathrm{S}(1)-\mathrm{C}(13)-\mathrm{C}(18)-\mathrm{C}(17)$

$\mathrm{C}(16)-\mathrm{C}(17)-\mathrm{C}(18)-\mathrm{C}(13)$
$158.4(2)$

$-86.8(3)$

$0.9(4)$

$-179.4(2)$

$-0.8(5)$

$0.5(5)$

$-180.0(2)$

$-0.3(5)$

$-179.9(2)$

$-0.7(4)$

$179.6(2)$

$0.4(4)$

\section{(2) Single-crystal X-ray crystallography of 12}

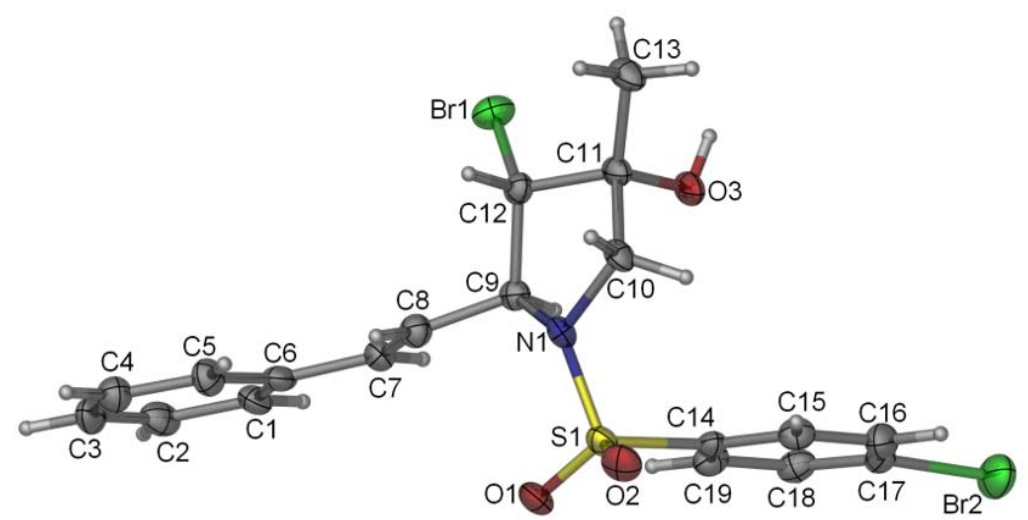

Data intensity of 12 was collected using a Bruker SMART APEX II (Mo radiation). The X-ray condition of was $50 \mathrm{kV} \times 30 \mathrm{~mA}$. Data collection and reduction were done by using the Bruker ApexII software package. The structure was solved by direct methods and refined by full-matrix least-squares on F2 with anisotropic displacement parameters for non-H atoms using SHELX-97. Hydrogen atoms were added at their geometrically idea positions and refined isotropically. Crystal data for 12: $\mathrm{C}_{19} \mathrm{H}_{19} \mathrm{Br}_{2} \mathrm{NO}_{3} \mathrm{~S}, \mathrm{M}=501.23 ; \mathrm{T}=173(2) \mathrm{K} ; \lambda=0.71073 \AA$; Monoclinic, $\mathrm{P} 2(1), \mathrm{a}=7.7418(4) \AA, \mathrm{b}=$ 12.0109(6) $\AA, \mathrm{c}=10.5954(6) \AA, \mathrm{V}=957.26(9) \AA^{\wedge} 3 ; \mathrm{z}=2, d \mathrm{calc}=1.739 \mathrm{Mg} / \mathrm{m}^{\wedge} 3,11037$ reflections measured, 3313 unique [Rint $=0.0308], \mathrm{R} 1=0.0269$, wR2 $=0.0522(I>2 \sigma)$, final R1 $=0.0242, \mathrm{wR} 2=$ $0.0507, \mathrm{GOF}=1.056$, and 235 parameters. 
Table S14. Crystal data and structure refinement for 12.

\begin{tabular}{|c|c|}
\hline Identification code & 12 \\
\hline Empirical formula & $\mathrm{C}_{19} \mathrm{H}_{19} \mathrm{Br}_{2} \mathrm{NO}_{3} \mathrm{~S}$ \\
\hline Formula weight & 501.23 \\
\hline Temperature & $173(2) \mathrm{K}$ \\
\hline Wavelength & $0.71073 \AA$ \\
\hline Crystal system, space group & Monoclinic, P2(1) \\
\hline Unit cell dimensions & $\begin{array}{cc}\mathrm{a}=7.7418(4) \AA & \text { alpha }=90 \mathrm{deg} . \\
\mathrm{b}=12.0109(6) \AA & \text { beta }=103.683(2) \mathrm{deg} . \\
\mathrm{c}=10.5954(6) \AA \quad \text { gamma }=90 \mathrm{deg} .\end{array}$ \\
\hline Volume & $957.26(9) \AA^{\wedge} 3$ \\
\hline Z, Calculated density & $2,1.739 \mathrm{Mg} / \mathrm{m}^{\wedge} 3$ \\
\hline Absorption coefficient & $4.362 \mathrm{~mm}^{\wedge}-1$ \\
\hline $\mathrm{F}(000)$ & 500 \\
\hline Crystal size & $0.18 \times 0.16 \times 0.11 \mathrm{~mm}$ \\
\hline Theta range for data collection & 2.71 to $25.00 \mathrm{deg}$. \\
\hline Limiting indices & $-9<=\mathrm{h}<=8,-14<=\mathrm{k}<=14,-12<=1<=11$ \\
\hline Reflections collected / unique & $11037 / 3313[\mathrm{R}(\mathrm{int})=0.0308]$ \\
\hline Completeness to theta $=25.00$ & $99.6 \%$ \\
\hline Absorption correction & Semi-empirical from equivalents \\
\hline Max. and min. transmission & 0.6454 and 0.5073 \\
\hline Refinement method & Full-matrix least-squares on $\mathrm{F}^{\wedge} 2$ \\
\hline Data / restraints / parameters & $3313 / 1 / 235$ \\
\hline Goodness-of-fit on $\mathrm{F}^{\wedge} 2$ & 1.056 \\
\hline Final $\mathrm{R}$ indices [I $>2 \operatorname{sigma}(\mathrm{I})]$ & $\mathrm{R} 1=0.0242, \mathrm{wR} 2=0.0507$ \\
\hline $\mathrm{R}$ indices (all data) & $\mathrm{R} 1=0.0269, \mathrm{wR} 2=0.0522$ \\
\hline Absolute structure parameter & $0.014(7)$ \\
\hline Largest diff. peak and hole & 0.397 and -0.234 e. $\AA^{\wedge} \wedge-3$ \\
\hline
\end{tabular}

Table S15. Atomic coordinates $\left(\mathrm{x} 10^{\wedge} 4\right)$ and equivalent isotropic displacement parameters $\left(\AA^{\wedge} 2 \mathrm{x}\right.$ $10^{\wedge} 3$ ) for 12.

$\mathrm{U}(\mathrm{eq})$ was defined as one third of the trace of the orthogonalized Uij tensor.

\begin{tabular}{lcccc}
\hline & $\mathrm{x}$ & $\mathrm{y}$ & $\mathrm{z}$ & $\mathrm{U}(\mathrm{eq})$ \\
\hline $\mathrm{Br}(1)$ & $19811(1)$ & $2837(1)$ & $13339(1)$ & $32(1)$ \\
$\mathrm{Br}(2)$ & $19563(1)$ & $-356(1)$ & $20708(1)$ & $42(1)$ \\
$\mathrm{S}(1)$ & $14686(1)$ & $2461(1)$ & $16098(1)$ & $22(1)$ \\
$\mathrm{O}(1)$ & $13700(3)$ & $1659(2)$ & $15226(2)$ & $28(1)$ \\
$\mathrm{O}(2)$ & $13773(3)$ & $3271(2)$ & $16693(2)$ & $30(1)$ \\
$\mathrm{O}(3)$ & $19800(3)$ & $3026(2)$ & $16349(2)$ & $27(1)$ \\
$\mathrm{N}(1)$ & $15880(4)$ & $3155(2)$ & $15296(3)$ & $22(1)$ \\
$\mathrm{C}(1)$ & $14538(4)$ & $108(3)$ & $10569(3)$ & $26(1)$ \\
$\mathrm{C}(2)$ & $13594(5)$ & $-148(3)$ & $9299(3)$ & $30(1)$
\end{tabular}




\begin{tabular}{lrrrr}
\hline & & & & \\
$\mathrm{C}(3)$ & $12448(5)$ & $632(3)$ & $8591(3)$ & $33(1)$ \\
$\mathrm{C}(4)$ & $12220(5)$ & $1649(3)$ & $9125(3)$ & $30(1)$ \\
$\mathrm{C}(5)$ & $13134(5)$ & $1891(3)$ & $10380(3)$ & $28(1)$ \\
$\mathrm{C}(6)$ & $14314(4)$ & $1133(3)$ & $11113(3)$ & $24(1)$ \\
$\mathrm{C}(7)$ & $15379(4)$ & $1382(3)$ & $12430(3)$ & $23(1)$ \\
$\mathrm{C}(8)$ & $15390(5)$ & $2317(3)$ & $13088(3)$ & $24(1)$ \\
$\mathrm{C}(9)$ & $16693(4)$ & $2565(2)$ & $14334(3)$ & $21(1)$ \\
$\mathrm{C}(10)$ & $17112(4)$ & $4034(3)$ & $15998(3)$ & $22(1)$ \\
$\mathrm{C}(11)$ & $18830(4)$ & $3879(2)$ & $15556(3)$ & $22(1)$ \\
$\mathrm{C}(12)$ & $18056(4)$ & $3439(3)$ & $14186(3)$ & $22(1)$ \\
$\mathrm{C}(13)$ & $19917(5)$ & $4931(3)$ & $15594(3)$ & $28(1)$ \\
$\mathrm{C}(14)$ & $16136(4)$ & $1721(3)$ & $17361(3)$ & $20(1)$ \\
$\mathrm{C}(15)$ & $16660(5)$ & $2181(2)$ & $18590(3)$ & $25(1)$ \\
$\mathrm{C}(16)$ & $17717(5)$ & $1580(3)$ & $19581(3)$ & $29(1)$ \\
$\mathrm{C}(17)$ & $18234(4)$ & $517(3)$ & $19326(3)$ & $26(1)$ \\
$\mathrm{C}(18)$ & $17770(5)$ & $63(3)$ & $18103(3)$ & $26(1)$ \\
$\mathrm{C}(19)$ & $16695(5)$ & $662(3)$ & $17106(3)$ & $26(1)$ \\
& & & & \\
\hline
\end{tabular}

Table S16. Bond lengths $[\AA]$ and angles [deg] for 12 .

\begin{tabular}{lc}
\hline $\mathrm{Br}(1)-\mathrm{C}(12)$ & $1.937(3)$ \\
$\mathrm{Br}(2)-\mathrm{C}(17)$ & $1.895(3)$ \\
$\mathrm{S}(1)-\mathrm{O}(1)$ & $1.425(2)$ \\
$\mathrm{S}(1)-\mathrm{O}(2)$ & $1.433(2)$ \\
$\mathrm{S}(1)-\mathrm{N}(1)$ & $1.626(3)$ \\
$\mathrm{S}(1)-\mathrm{C}(14)$ & $1.769(3)$ \\
$\mathrm{O}(3)-\mathrm{C}(11)$ & $1.422(4)$ \\
$\mathrm{O}(3)-\mathrm{H}(3 \mathrm{~B})$ & 0.8400 \\
$\mathrm{~N}(1)-\mathrm{C}(9)$ & $1.497(4)$ \\
$\mathrm{N}(1)-\mathrm{C}(10)$ & $1.498(4)$ \\
$\mathrm{C}(1)-\mathrm{C}(6)$ & $1.387(5)$ \\
$\mathrm{C}(1)-\mathrm{C}(2)$ & $1.406(5)$ \\
$\mathrm{C}(1)-\mathrm{H}(1 \mathrm{~A})$ & 0.9500 \\
$\mathrm{C}(2)-\mathrm{C}(3)$ & $1.383(5)$ \\
$\mathrm{C}(2)-\mathrm{H}(2 \mathrm{~A})$ & 0.9500 \\
$\mathrm{C}(3)-\mathrm{C}(4)$ & $1.376(5)$ \\
$\mathrm{C}(3)-\mathrm{H}(3 \mathrm{~A})$ & 0.9500 \\
$\mathrm{C}(4)-\mathrm{C}(5)$ & $1.381(5)$ \\
$\mathrm{C}(4)-\mathrm{H}(4 \mathrm{~A})$ & 0.9500 \\
$\mathrm{C}(5)-\mathrm{C}(6)$ & $1.389(5)$ \\
$\mathrm{C}(5)-\mathrm{H}(5 \mathrm{~A})$ & 0.9500 \\
$\mathrm{C}(6)-\mathrm{C}(7)$ & $1.473(5)$ \\
$\mathrm{C}(7)-\mathrm{C}(8)$ & $1.321(4)$ \\
$\mathrm{C}(7)-\mathrm{H}(7 \mathrm{~A})$ & 0.9500 \\
$\mathrm{C}(8)-\mathrm{C}(9)$ & $1.490(5)$ \\
$\mathrm{C}(8)-\mathrm{H}(8 \mathrm{~A})$ & 0.9500 \\
& \\
&
\end{tabular}




\begin{tabular}{|c|c|}
\hline$C(9)-C(12)$ & $1.523(4)$ \\
\hline $\mathrm{C}(9)-\mathrm{H}(9 \mathrm{~A})$ & 1.0000 \\
\hline $\mathrm{C}(10)-\mathrm{C}(11)$ & $1.522(5)$ \\
\hline $\mathrm{C}(10)-\mathrm{H}(10 \mathrm{~A})$ & 0.9900 \\
\hline $\mathrm{C}(10)-\mathrm{H}(10 \mathrm{~B})$ & 0.9900 \\
\hline $\mathrm{C}(11)-\mathrm{C}(13)$ & $1.513(4)$ \\
\hline $\mathrm{C}(11)-\mathrm{C}(12)$ & $1.528(5)$ \\
\hline $\mathrm{C}(12)-\mathrm{H}(12 \mathrm{~A})$ & 1.0000 \\
\hline $\mathrm{C}(13)-\mathrm{H}(13 \mathrm{~A})$ & 0.9800 \\
\hline $\mathrm{C}(13)-\mathrm{H}(13 \mathrm{~B})$ & 0.9800 \\
\hline $\mathrm{C}(13)-\mathrm{H}(13 \mathrm{C})$ & 0.9800 \\
\hline$C(14)-C(15)$ & $1.384(5)$ \\
\hline C(14)-C(19) & $1.391(5)$ \\
\hline$C(15)-C(16)$ & $1.374(5)$ \\
\hline $\mathrm{C}(15)-\mathrm{H}(15 \mathrm{~A})$ & 0.9500 \\
\hline $\mathrm{C}(16)-\mathrm{C}(17)$ & $1.384(5)$ \\
\hline $\mathrm{C}(16)-\mathrm{H}(16 \mathrm{~A})$ & 0.9500 \\
\hline$C(17)-C(18)$ & $1.372(5)$ \\
\hline $\mathrm{C}(18)-\mathrm{C}(19)$ & $1.383(5)$ \\
\hline $\mathrm{C}(18)-\mathrm{H}(18 \mathrm{~A})$ & 0.9500 \\
\hline $\mathrm{C}(19)-\mathrm{H}(19 \mathrm{~A})$ & 0.9500 \\
\hline $\mathrm{O}(1)-\mathrm{S}(1)-\mathrm{O}(2)$ & $119.97(15)$ \\
\hline $\mathrm{O}(1)-\mathrm{S}(1)-\mathrm{N}(1)$ & $106.92(14)$ \\
\hline $\mathrm{O}(2)-\mathrm{S}(1)-\mathrm{N}(1)$ & $106.40(14)$ \\
\hline $\mathrm{O}(1)-\mathrm{S}(1)-\mathrm{C}(14)$ & $107.32(15)$ \\
\hline $\mathrm{O}(2)-\mathrm{S}(1)-\mathrm{C}(14)$ & $107.40(15)$ \\
\hline $\mathrm{N}(1)-\mathrm{S}(1)-\mathrm{C}(14)$ & $108.41(15)$ \\
\hline $\mathrm{C}(11)-\mathrm{O}(3)-\mathrm{H}(3 \mathrm{~B})$ & 109.5 \\
\hline $\mathrm{C}(9)-\mathrm{N}(1)-\mathrm{C}(10)$ & $110.6(3)$ \\
\hline $\mathrm{C}(9)-\mathrm{N}(1)-\mathrm{S}(1)$ & $119.71(19)$ \\
\hline $\mathrm{C}(10)-\mathrm{N}(1)-\mathrm{S}(1)$ & $118.4(2)$ \\
\hline$C(6)-C(1)-C(2)$ & $120.3(3)$ \\
\hline $\mathrm{C}(6)-\mathrm{C}(1)-\mathrm{H}(1 \mathrm{~A})$ & 119.9 \\
\hline $\mathrm{C}(2)-\mathrm{C}(1)-\mathrm{H}(1 \mathrm{~A})$ & 119.9 \\
\hline$C(3)-C(2)-C(1)$ & $119.5(3)$ \\
\hline $\mathrm{C}(3)-\mathrm{C}(2)-\mathrm{H}(2 \mathrm{~A})$ & 120.2 \\
\hline $\mathrm{C}(1)-\mathrm{C}(2)-\mathrm{H}(2 \mathrm{~A})$ & 120.2 \\
\hline$C(4)-C(3)-C(2)$ & $120.5(3)$ \\
\hline $\mathrm{C}(4)-\mathrm{C}(3)-\mathrm{H}(3 \mathrm{~A})$ & 119.8 \\
\hline $\mathrm{C}(2)-\mathrm{C}(3)-\mathrm{H}(3 \mathrm{~A})$ & 119.8 \\
\hline$C(3)-C(4)-C(5)$ & $119.7(3)$ \\
\hline $\mathrm{C}(3)-\mathrm{C}(4)-\mathrm{H}(4 \mathrm{~A})$ & 120.1 \\
\hline $\mathrm{C}(5)-\mathrm{C}(4)-\mathrm{H}(4 \mathrm{~A})$ & 120.1 \\
\hline$C(4)-C(5)-C(6)$ & $121.4(3)$ \\
\hline $\mathrm{C}(4)-\mathrm{C}(5)-\mathrm{H}(5 \mathrm{~A})$ & 119.3 \\
\hline $\mathrm{C}(6)-\mathrm{C}(5)-\mathrm{H}(5 \mathrm{~A})$ & 119.3 \\
\hline
\end{tabular}




\begin{tabular}{|c|c|}
\hline$C(1)-C(6)-C(5)$ & $118.7(3)$ \\
\hline$C(1)-C(6)-C(7)$ & $118.4(3)$ \\
\hline$C(5)-C(6)-C(7)$ & $122.9(3)$ \\
\hline $\mathrm{C}(8)-\mathrm{C}(7)-\mathrm{C}(6)$ & $127.2(3)$ \\
\hline $\mathrm{C}(8)-\mathrm{C}(7)-\mathrm{H}(7 \mathrm{~A})$ & 116.4 \\
\hline $\mathrm{C}(6)-\mathrm{C}(7)-\mathrm{H}(7 \mathrm{~A})$ & 116.4 \\
\hline $\mathrm{C}(7)-\mathrm{C}(8)-\mathrm{C}(9)$ & $123.8(3)$ \\
\hline $\mathrm{C}(7)-\mathrm{C}(8)-\mathrm{H}(8 \mathrm{~A})$ & 118.1 \\
\hline $\mathrm{C}(9)-\mathrm{C}(8)-\mathrm{H}(8 \mathrm{~A})$ & 118.1 \\
\hline $\mathrm{C}(8)-\mathrm{C}(9)-\mathrm{N}(1)$ & 113.1(3) \\
\hline $\mathrm{C}(8)-\mathrm{C}(9)-\mathrm{C}(12)$ & $112.8(3)$ \\
\hline $\mathrm{N}(1)-\mathrm{C}(9)-\mathrm{C}(12)$ & $99.0(2)$ \\
\hline $\mathrm{C}(8)-\mathrm{C}(9)-\mathrm{H}(9 \mathrm{~A})$ & 110.5 \\
\hline $\mathrm{N}(1)-\mathrm{C}(9)-\mathrm{H}(9 \mathrm{~A})$ & 110.5 \\
\hline $\mathrm{C}(12)-\mathrm{C}(9)-\mathrm{H}(9 \mathrm{~A})$ & 110.5 \\
\hline $\mathrm{N}(1)-\mathrm{C}(10)-\mathrm{C}(11)$ & $105.1(2)$ \\
\hline $\mathrm{N}(1)-\mathrm{C}(10)-\mathrm{H}(10 \mathrm{~A})$ & 110.7 \\
\hline $\mathrm{C}(11)-\mathrm{C}(10)-\mathrm{H}(10 \mathrm{~A})$ & 110.7 \\
\hline $\mathrm{N}(1)-\mathrm{C}(10)-\mathrm{H}(10 \mathrm{~B})$ & 110.7 \\
\hline $\mathrm{C}(11)-\mathrm{C}(10)-\mathrm{H}(10 \mathrm{~B})$ & 110.7 \\
\hline $\mathrm{H}(10 \mathrm{~A})-\mathrm{C}(10)-\mathrm{H}(10 \mathrm{~B})$ & 108.8 \\
\hline $\mathrm{O}(3)-\mathrm{C}(11)-\mathrm{C}(13)$ & $111.9(3)$ \\
\hline $\mathrm{O}(3)-\mathrm{C}(11)-\mathrm{C}(10)$ & $106.5(2)$ \\
\hline$C(13)-C(11)-C(10)$ & $114.2(3)$ \\
\hline $\mathrm{O}(3)-\mathrm{C}(11)-\mathrm{C}(12)$ & $110.2(2)$ \\
\hline $\mathrm{C}(13)-\mathrm{C}(11)-\mathrm{C}(12)$ & $113.9(3)$ \\
\hline $\mathrm{C}(10)-\mathrm{C}(11)-\mathrm{C}(12)$ & $99.2(3)$ \\
\hline$C(9)-C(12)-C(11)$ & $105.4(3)$ \\
\hline$C(9)-C(12)-B r(1)$ & $111.6(2)$ \\
\hline $\mathrm{C}(11)-\mathrm{C}(12)-\mathrm{Br}(1)$ & $114.1(2)$ \\
\hline $\mathrm{C}(9)-\mathrm{C}(12)-\mathrm{H}(12 \mathrm{~A})$ & 108.5 \\
\hline $\mathrm{C}(11)-\mathrm{C}(12)-\mathrm{H}(12 \mathrm{~A})$ & 108.5 \\
\hline $\mathrm{Br}(1)-\mathrm{C}(12)-\mathrm{H}(12 \mathrm{~A})$ & 108.5 \\
\hline $\mathrm{C}(11)-\mathrm{C}(13)-\mathrm{H}(13 \mathrm{~A})$ & 109.5 \\
\hline $\mathrm{C}(11)-\mathrm{C}(13)-\mathrm{H}(13 \mathrm{~B})$ & 109.5 \\
\hline $\mathrm{H}(13 \mathrm{~A})-\mathrm{C}(13)-\mathrm{H}(13 \mathrm{~B})$ & 109.5 \\
\hline $\mathrm{C}(11)-\mathrm{C}(13)-\mathrm{H}(13 \mathrm{C})$ & 109.5 \\
\hline $\mathrm{H}(13 \mathrm{~A})-\mathrm{C}(13)-\mathrm{H}(13 \mathrm{C})$ & 109.5 \\
\hline $\mathrm{H}(13 \mathrm{~B})-\mathrm{C}(13)-\mathrm{H}(13 \mathrm{C})$ & 109.5 \\
\hline$C(15)-C(14)-C(19)$ & $120.8(3)$ \\
\hline $\mathrm{C}(15)-\mathrm{C}(14)-\mathrm{S}(1)$ & $120.2(2)$ \\
\hline C(19)-C(14)-S(1) & $118.9(3)$ \\
\hline$C(16)-C(15)-C(14)$ & $119.9(3)$ \\
\hline $\mathrm{C}(16)-\mathrm{C}(15)-\mathrm{H}(15 \mathrm{~A})$ & 120.0 \\
\hline $\mathrm{C}(14)-\mathrm{C}(15)-\mathrm{H}(15 \mathrm{~A})$ & 120.0 \\
\hline$C(15)-C(16)-C(17)$ & $118.9(3)$ \\
\hline $\mathrm{C}(15)-\mathrm{C}(16)-\mathrm{H}(16 \mathrm{~A})$ & 120.6 \\
\hline
\end{tabular}




$\begin{array}{lc}\mathrm{C}(17)-\mathrm{C}(16)-\mathrm{H}(16 \mathrm{~A}) & 120.6 \\ \mathrm{C}(18)-\mathrm{C}(17)-\mathrm{C}(16) & 121.9(3) \\ \mathrm{C}(18)-\mathrm{C}(17)-\mathrm{Br}(2) & 118.9(3) \\ \mathrm{C}(16)-\mathrm{C}(17)-\mathrm{Br}(2) & 119.2(3) \\ \mathrm{C}(17)-\mathrm{C}(18)-\mathrm{C}(19) & 119.4(3) \\ \mathrm{C}(17)-\mathrm{C}(18)-\mathrm{H}(18 \mathrm{~A}) & 120.3 \\ \mathrm{C}(19)-\mathrm{C}(18)-\mathrm{H}(18 \mathrm{~A}) & 120.3 \\ \mathrm{C}(18)-\mathrm{C}(19)-\mathrm{C}(14) & 119.1(3) \\ \mathrm{C}(18)-\mathrm{C}(19)-\mathrm{H}(19 \mathrm{~A}) & 120.5 \\ \mathrm{C}(14)-\mathrm{C}(19)-\mathrm{H}(19 \mathrm{~A}) & 120.5\end{array}$

Symmetry transformations used to generate equivalent atoms:

Table S17. Anisotropic displacement parameters $\left(\AA^{\wedge} 2 \times 10^{\wedge} 3\right)$ for 12. The anisotropic displacement factor exponent takes the form: $-2 \mathrm{pi}^{\wedge} 2\left[\mathrm{~h}^{\wedge} 2 \mathrm{a}^{* \wedge} 2 \mathrm{U} 11+\ldots+2 \mathrm{~h} \mathrm{k} \mathrm{a*} \mathrm{b}^{*} \mathrm{U} 12\right]$

\begin{tabular}{lrrrrrr}
\hline & $\mathrm{U} 11$ & $\mathrm{U} 22$ & $\mathrm{U} 33$ & $\mathrm{U} 23$ & $\mathrm{U} 13$ & $\mathrm{U} 12$ \\
\hline $\mathrm{Br}(1)$ & $32(1)$ & $30(1)$ & $37(1)$ & $2(1)$ & $16(1)$ & $-1(1)$ \\
$\mathrm{Br}(2)$ & $36(1)$ & $48(1)$ & $38(1)$ & $18(1)$ & $3(1)$ & $8(1)$ \\
$\mathrm{S}(1)$ & $15(1)$ & $25(1)$ & $25(1)$ & $0(1)$ & $2(1)$ & $0(1)$ \\
$\mathrm{O}(1)$ & $19(1)$ & $32(1)$ & $30(1)$ & $-1(1)$ & $0(1)$ & $-6(1)$ \\
$\mathrm{O}(2)$ & $20(1)$ & $32(1)$ & $37(2)$ & $1(1)$ & $8(1)$ & $6(1)$ \\
$\mathrm{O}(3)$ & $20(1)$ & $27(1)$ & $29(1)$ & $10(1)$ & $-4(1)$ & $0(1)$ \\
$\mathrm{N}(1)$ & $18(2)$ & $24(1)$ & $21(2)$ & $-2(1)$ & $0(1)$ & $-1(1)$ \\
$\mathrm{C}(1)$ & $22(2)$ & $29(2)$ & $27(2)$ & $-3(1)$ & $5(2)$ & $-8(1)$ \\
$\mathrm{C}(2)$ & $29(2)$ & $30(2)$ & $33(2)$ & $-10(2)$ & $10(2)$ & $-6(2)$ \\
$\mathrm{C}(3)$ & $31(2)$ & $50(2)$ & $18(2)$ & $-6(2)$ & $5(2)$ & $-10(2)$ \\
$\mathrm{C}(4)$ & $27(2)$ & $34(2)$ & $28(2)$ & $2(2)$ & $6(2)$ & $0(2)$ \\
$\mathrm{C}(5)$ & $29(2)$ & $29(2)$ & $23(2)$ & $-5(1)$ & $4(2)$ & $-2(2)$ \\
$\mathrm{C}(6)$ & $20(2)$ & $26(2)$ & $26(2)$ & $-2(1)$ & $8(2)$ & $-8(2)$ \\
$\mathrm{C}(7)$ & $20(2)$ & $27(2)$ & $22(2)$ & $1(1)$ & $3(2)$ & $-2(1)$ \\
$\mathrm{C}(8)$ & $23(2)$ & $25(2)$ & $24(2)$ & $2(1)$ & $5(2)$ & $2(2)$ \\
$\mathrm{C}(9)$ & $19(2)$ & $19(2)$ & $23(2)$ & $3(1)$ & $4(1)$ & $2(1)$ \\
$\mathrm{C}(10)$ & $21(2)$ & $19(2)$ & $24(2)$ & $-4(1)$ & $0(2)$ & $-3(1)$ \\
$\mathrm{C}(11)$ & $18(2)$ & $22(2)$ & $24(2)$ & $6(1)$ & $0(2)$ & $0(1)$ \\
$\mathrm{C}(12)$ & $20(2)$ & $24(2)$ & $20(2)$ & $4(1)$ & $4(2)$ & $2(1)$ \\
$\mathrm{C}(13)$ & $24(2)$ & $22(2)$ & $36(2)$ & $2(1)$ & $0(2)$ & $-4(1)$ \\
$\mathrm{C}(14)$ & $16(2)$ & $25(2)$ & $20(2)$ & $4(1)$ & $7(1)$ & $-1(1)$ \\
$\mathrm{C}(15)$ & $26(2)$ & $20(2)$ & $29(2)$ & $0(1)$ & $9(2)$ & $0(1)$ \\
$\mathrm{C}(16)$ & $31(2)$ & $31(2)$ & $23(2)$ & $-2(2)$ & $6(2)$ & $-6(2)$ \\
$\mathrm{C}(17)$ & $16(2)$ & $37(2)$ & $24(2)$ & $11(2)$ & $4(2)$ & $0(2)$ \\
& & & & & &
\end{tabular}




$\begin{array}{llllrrr}\mathrm{C}(18) & 26(2) & 24(2) & 32(2) & 4(1) & 13(2) & 3(1) \\ \mathrm{C}(19) & 29(2) & 24(2) & 25(2) & -1(1) & 10(2) & -3(2)\end{array}$

Table S18. Hydrogen coordinates $\left(\times 10^{4}\right)$ and isotropic displacement parameters $\left(\AA^{\wedge} 2 \times 10^{3}\right)$ for 12.

\begin{tabular}{lcrrc}
\hline & $x$ & $y$ & $z$ & $U(e q)$ \\
\hline$H(3 B)$ & 20894 & 3123 & 16421 & 40 \\
$H(1 A)$ & 15332 & -423 & 11058 & 31 \\
$\mathrm{H}(2 \mathrm{~A})$ & 13742 & -852 & 8929 & 36 \\
$\mathrm{H}(3 \mathrm{~A})$ & 11814 & 464 & 7730 & 40 \\
$\mathrm{H}(4 \mathrm{~A})$ & 11436 & 2184 & 8633 & 36 \\
$\mathrm{H}(5 \mathrm{~A})$ & 12953 & 2589 & 10750 & 33 \\
$\mathrm{H}(7 \mathrm{~A})$ & 16141 & 806 & 12852 & 28 \\
$\mathrm{H}(8 \mathrm{~A})$ & 14516 & 2863 & 12747 & 29 \\
$\mathrm{H}(9 \mathrm{~A})$ & 17301 & 1867 & 14722 & 25 \\
$\mathrm{H}(10 \mathrm{~A})$ & 17322 & 3936 & 16950 & 27 \\
$\mathrm{H}(10 \mathrm{~B})$ & 16613 & 4786 & 15766 & 27 \\
$\mathrm{H}(12 \mathrm{~A})$ & 17420 & 4063 & 13643 & 26 \\
$\mathrm{H}(13 \mathrm{~A})$ & 20369 & 5166 & 16498 & 42 \\
$\mathrm{H}(13 \mathrm{~B})$ & 19168 & 5521 & 15111 & 42 \\
$\mathrm{H}(13 \mathrm{C})$ & 20918 & 4788 & 15198 & 42 \\
$\mathrm{H}(15 \mathrm{~A})$ & 16288 & 2911 & 18749 & 30 \\
$\mathrm{H}(16 \mathrm{~A})$ & 18088 & 1889 & 20428 & 34 \\
$\mathrm{H}(18 \mathrm{~A})$ & 18184 & -656 & 17944 & 32 \\
$\mathrm{H}(19 \mathrm{~A})$ & 16342 & 354 & 16258 & 31 \\
& & & & \\
\hline
\end{tabular}

Table S19. Torsion angles [deg] for 12.

\begin{tabular}{lr}
\hline $\mathrm{O}(1)-\mathrm{S}(1)-\mathrm{N}(1)-\mathrm{C}(9)$ & $-37.6(3)$ \\
$\mathrm{O}(2)-\mathrm{S}(1)-\mathrm{N}(1)-\mathrm{C}(9)$ & $-166.9(2)$ \\
$\mathrm{C}(14)-\mathrm{S}(1)-\mathrm{N}(1)-\mathrm{C}(9)$ & $77.8(3)$ \\
$\mathrm{O}(1)-\mathrm{S}(1)-\mathrm{N}(1)-\mathrm{C}(10)$ & $-177.7(2)$ \\
$\mathrm{O}(2)-\mathrm{S}(1)-\mathrm{N}(1)-\mathrm{C}(10)$ & $52.9(3)$ \\
$\mathrm{C}(14)-\mathrm{S}(1)-\mathrm{N}(1)-\mathrm{C}(10)$ & $-62.3(3)$ \\
$\mathrm{C}(6)-\mathrm{C}(1)-\mathrm{C}(2)-\mathrm{C}(3)$ & $0.4(5)$ \\
$\mathrm{C}(1)-\mathrm{C}(2)-\mathrm{C}(3)-\mathrm{C}(4)$ & $-0.4(5)$ \\
$\mathrm{C}(2)-\mathrm{C}(3)-\mathrm{C}(4)-\mathrm{C}(5)$ & $-0.4(5)$ \\
$\mathrm{C}(3)-\mathrm{C}(4)-\mathrm{C}(5)-\mathrm{C}(6)$ & $1.3(5)$ \\
$\mathrm{C}(2)-\mathrm{C}(1)-\mathrm{C}(6)-\mathrm{C}(5)$ & $0.5(5)$ \\
$\mathrm{C}(2)-\mathrm{C}(1)-\mathrm{C}(6)-\mathrm{C}(7)$ & $-177.8(3)$ \\
$\mathrm{C}(4)-\mathrm{C}(5)-\mathrm{C}(6)-\mathrm{C}(1)$ & $-1.3(5)$ \\
$\mathrm{C}(4)-\mathrm{C}(5)-\mathrm{C}(6)-\mathrm{C}(7)$ & $176.9(3)$ \\
$\mathrm{C}(1)-\mathrm{C}(6)-\mathrm{C}(7)-\mathrm{C}(8)$ & $177.8(3)$
\end{tabular}




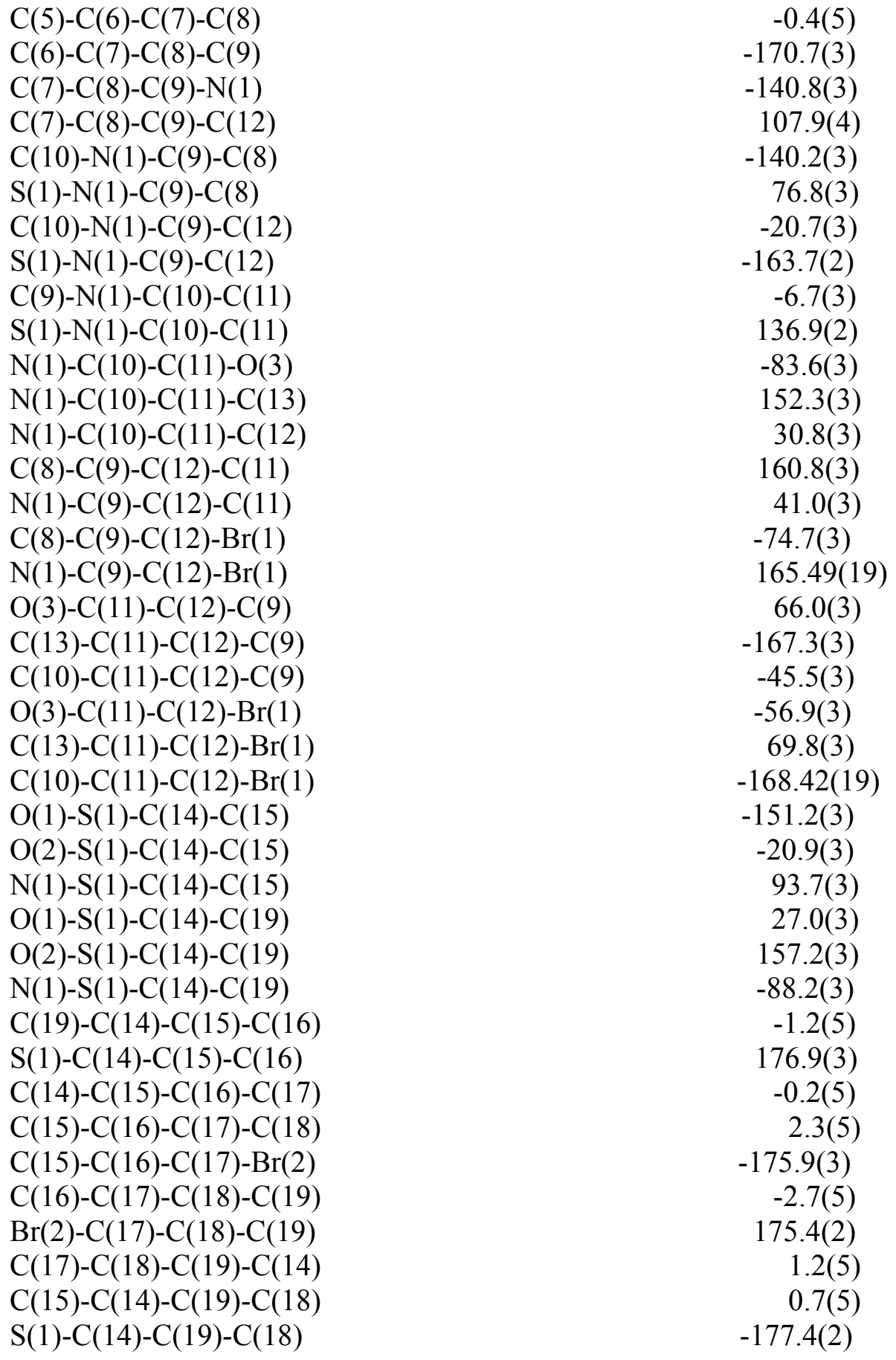




\section{Details for DFT calculations}

$(R, R)-\mathbf{1}$

Cartesian Coordinates (Angstroms)

C, $0,3.4444277721,-2.2066182264,-1.3405861968$

C, $0,2.1026161705,-2.1890381161,-0.9735454528$

C, $0,1.3399405455,-1.0232519644,-1.308361564$

C, $0,2.0087815869,0.0545726247,-1.9598942806$

C, $0,3.3820701727,-0.0307116111,-2.2923760132$

C, $0,4.1256110157,-1.1571910665,-2.0049871975$

$\mathrm{H}, 0,4.0190081887,-3.0929356678,-1.1005154592$

H,0,3.8322910562,0.823719023,-2.7890358142

C, $0,1.3393855572,1.2873883326,-2.2551638825$

$\mathrm{N}, 0,0.0782608765,1.5207852626,-2.0684843137$

$\mathrm{N}, 0,-2.142715542,1.8633745687,-0.7838581026$

C, $0,-3.2990759492,1.9075060582,-0.180191273$

C, $0,-4.1257243484,0.8007773726,0.1775278588$

C, $0,-3.6986488613,-0.5505230674,-0.0042544899$

C, $0,-5.3928233384,1.0997697243,0.7419199312$

C, $0,-4.5955745308,-1.6027395412,0.3851819312$

C, $0,-6.2662562734,0.1029668862,1.1173568019$

$\mathrm{H}, 0,-5.6547746659,2.1469179612,0.8611297751$

C, $0,-5.8242130783,-1.2313051775,0.9159175203$

$\mathrm{H}, 0,-6.5067775457,-2.0224582641,1.2012808946$

$\mathrm{O}, 0,-2.509744729,-0.8173895312,-0.4883262933$

$\mathrm{O}, 0,0.0586575596,-0.9489328035,-0.9961381252$

Al,0,-1.3118424665,0.1517483253,-1.4955777956

$\mathrm{H}, 0,1.9715169063,2.082288973,-2.6576640285$

$\mathrm{H}, 0,-3.7037803899,2.8848985967,0.0888978531$

C, $0,-1.3947760969,3.1102103095,-1.0630839755$

C, $0,-0.5377158164,2.8260595595,-2.3194692702$

C, $0,-2.2201488399,4.3919056361,-1.2458776596$

C, $0,0.3868944928,4.0010696302,-2.6477503563$

$\mathrm{H}, 0,-1.230794945,2.6780593518,-3.1607997746$

C, $0,-1.3077310234,5.5785031702,-1.6042996107$

$\mathrm{H}, 0,-2.9621044296,4.2310124537,-2.0403655379$

C, $0,-0.4373951011,5.2855232501,-2.8335189059$

H,0,0.956887316,3.7927393294,-3.5611159986

$\mathrm{H}, 0,1.1153581203,4.1415657825,-1.8358519378$

$\mathrm{H}, 0,-1.9207682524,6.4709271266,-1.7768310112$

$\mathrm{H}, 0,-0.6596783807,5.8070910912,-0.7459259604$

$\mathrm{H}, 0,0.2322691291,6.1302585131,-3.0334149413$

$\mathrm{H}, 0,-1.0794647778,5.1777993727,-3.718718181$

$\mathrm{H}, 0,-0.6942120797,3.2594209102,-0.2259153969$

$\mathrm{H}, 0,-2.7739277495,4.6355111286,-0.3321396793$ 
C,0,-4.2103395956,-3.0859790578,0.2127091029 C, $0,-2.9554344388,-3.3957668622,1.0632820658$ $\mathrm{H}, 0,-3.1579115802,-3.2267780119,2.1283912145$ $\mathrm{H}, 0,-2.6686408264,-4.4475585909,0.9383888469$ $\mathrm{H}, 0,-2.112007716,-2.7720596238,0.7674173754$ C, $0,-5.3302412932,-4.0393203177,0.6784924087$ $\mathrm{H}, 0,-5.0002400032,-5.0739442394,0.5322130873$ $\mathrm{H}, 0,-5.5645981033,-3.918133769,1.742966464$ $\mathrm{H}, 0,-6.2540117809,-3.9071176208,0.1026520713$ C, $0,-3.9353310957,-3.3926031182,-1.2798596804$ $\mathrm{H}, 0,-4.8352807076,-3.2194558544,-1.8820844116$ $\mathrm{H}, 0,-3.1341929443,-2.7720320026,-1.6820276777$ $\mathrm{H}, 0,-3.6503135374,-4.4457199043,-1.3968186468$ C, $0,1.4593810495,-3.3802524298,-0.2347304274$ C, $0,0.935182106,-2.9115481337,1.1450776778$ H, $0,0.2009686219,-2.1113318936,1.0424563998$ $\mathrm{H}, 0,0.4611368541,-3.749520932,1.6709752858$ $\mathrm{H}, 0,1.7620746396,-2.5486086329,1.768334224$ C, $0,0.3016823245,-3.965074388,-1.0797754387$ $\mathrm{H}, 0,-0.4792380097,-3.2256886082,-1.2576727029$ $\mathrm{H}, 0,0.6724089129,-4.3187297857,-2.04948791$ $\mathrm{H}, 0,-0.145561533,-4.8205493777,-0.5583292735$ C,0,2.4643843977,-4.522260804,0.0205082871 $\mathrm{H}, 0,1.9543544221,-5.3336355415,0.5516178789$ $\mathrm{H}, 0,2.8621141364,-4.9415809944,-0.9114243161$ $\mathrm{H}, 0,3.3079889271,-4.2027250311,0.6440734$ C, $0,-7.6563035756,0.3654913482,1.7205003789$ C, $0,-7.7495034694,-0.2865001306,3.1208284156$ $\mathrm{H}, 0,-8.7396179625,-0.1111259349,3.5596904106$ $\mathrm{H}, 0,-7.5934119077,-1.3697005897,3.0790215994$ $\mathrm{H}, 0,-6.9973938318,0.1329224164,3.7989944881$ C,0,-7.944693079,1.8709126036,1.8742810108 $\mathrm{H}, 0,-7.2191642445,2.3581050877,2.5361900541$ $\mathrm{H}, 0,-7.9311308431,2.3890990811,0.9083351833$ $\mathrm{H}, 0,-8.9391686768,2.0147748117,2.3115467337$ C, $0,-8.7432630482,-0.2405151027,0.800393221$ $\mathrm{H}, 0,-8.7090384767,0.2138728964,-0.196388838$ $\mathrm{H}, 0,-8.6180686224,-1.3215293453,0.6779640486$ $\mathrm{H}, 0,-9.7418626892,-0.0671309853,1.2205842266$ C,0,5.6128191306,-1.3139671891,-2.364568786 C, $0,6.4398114002,-1.5577920699,-1.0794349343$ $\mathrm{H}, 0,6.1187405609,-2.462036006,-0.5516886547$ $\mathrm{H}, 0,7.5022660165,-1.6773733614,-1.3254671585$ $\mathrm{H}, 0,6.343927685,-0.7147145985,-0.3854358285$ C,0,6.1737801328,-0.0610606986,-3.0636095324 $\mathrm{H}, 0,5.6492308532,0.1497010853,-4.0027244063$ $\mathrm{H}, 0,6.104768635,0.8271053931,-2.4244905568$ 
$\mathrm{H}, 0,7.2322017672,-0.212951703,-3.30363258$

C, $0,5.7880788562,-2.5178358638,-3.3211565819$

$\mathrm{H}, 0,5.4331573822,-3.4511140906,-2.8709960912$

$\mathrm{H}, 0,5.2288717787,-2.3626973649,-4.2508726553$

$\mathrm{H}, 0,6.8461951707,-2.653341136,-3.5776737136$

$\mathrm{Cl}, 0,-2.1067854238,-0.1822403148,-3.5646548452$

$\mathrm{HF}=-2125.5328859$ (A.U.)

Zero-point correction $=0.821689$ (A.U.)

Total Gibbs free energy $=-2124.786247$ (A.U.)

$(R, R)-1$ (optimized in the solvent )

Cartesian Coordinates (Angstroms)

C, $0,3.4484056782,-2.2154470492,-1.3831440098$

C,0,2.1159214786,-2.1916766652,-0.9773051122

C, $0,1.3496547008,-1.0241144037,-1.2921232488$

C, $0,2.0019778205,0.0503970303,-1.9630827396$

C, $0,3.365097127,-0.0384880035,-2.3325384465$

C, $0,4.11326332,-1.169044727,-2.0661655823$

$\mathrm{H}, 0,4.0266573348,-3.1034341645,-1.1591487808$

$\mathrm{H}, 0,3.803525371,0.8155569407,-2.8391689613$

C, $0,1.3322351744,1.2905233275,-2.241725707$

$\mathrm{N}, 0,0.0738248305,1.5253948061,-2.039845893$

$\mathrm{N}, 0,-2.1434472424,1.8700562685,-0.7450013715$

C, $0,-3.3187722352,1.9177810584,-0.1830301532$

C, $0,-4.1508885949,0.8039348363,0.1567009426$

C, $0,-3.7048503787,-0.5447660405,0.0178639791$

C, $0,-5.4375455115,1.1007879721,0.6732931938$

C, $0,-4.5948147236,-1.5976783868,0.4153373426$

C, $0,-6.3120327156,0.1011286208,1.0465592499$

$\mathrm{H}, 0,-5.7139880945,2.1468395784,0.7600566891$

C, $0,-5.8463590567,-1.2302565926,0.8991765835$

$\mathrm{H}, 0,-6.5248862659,-2.0227042196,1.1898406095$

$\mathrm{O}, 0,-2.4975940704,-0.8107366259,-0.4362884143$

$\mathrm{O}, 0,0.0726418813,-0.9445582124,-0.944571951$

$\mathrm{Al}, 0,-1.2905930708,0.172475323,-1.416044381$

$\mathrm{H}, 0,1.9611099119,2.0858679671,-2.6442056418$

$\mathrm{H}, 0,-3.7327533227,2.8948815993,0.0666966367$

C, $0,-1.376758203,3.1150026255,-0.9969192455$

C, $0,-0.5311494952,2.846302343,-2.2629911479$

C, $0,-2.1820153214,4.4130040613,-1.1366098316$

C, $0,0.4091376731,4.0136200027,-2.5711920117$

$\mathrm{H}, 0,-1.2298539564,2.7258250521,-3.103771756$

C, $0,-1.2496886748,5.5912856831,-1.470985022$

$\mathrm{H}, 0,-2.9340000972,4.2901922237,-1.9282400185$

C,0,-0.3968136537,5.3149116021,-2.7159402093 
$\mathrm{H}, 0,0.9660303279,3.8198260457,-3.495282832$ $\mathrm{H}, 0,1.145375543,4.1207738246,-1.7622664688$ $\mathrm{H}, 0,-1.8496062855,6.497172473,-1.6148736875$ $\mathrm{H}, 0,-0.5907119636,5.7843646746,-0.6128460318$ H, $0,0.2874381372,6.1511674308,-2.8994217463$ $\mathrm{H}, 0,-1.0484738132,5.2412567974,-3.5974205651$ $\mathrm{H}, 0,-0.6731717678,3.2249466119,-0.1579436784$ $\mathrm{H}, 0,-2.7196025944,4.6382561362,-0.2091095607$ C, $0,-4.1831226735,-3.0811610474,0.310592392$ C, $0,-2.9502845907,-3.3407551064,1.2092718965$ $\mathrm{H}, 0,-3.1862948529,-3.1281741061,2.2595345104$ $\mathrm{H}, 0,-2.6502645213,-4.3940465354,1.1393116345$ $\mathrm{H}, 0,-2.10410405,-2.7215588835,0.9101753079$ C, $0,-5.3024135036,-4.0349409744,0.7773813856$ $\mathrm{H}, 0,-4.9523660467,-5.0688120218,0.6797417407$ $\mathrm{H}, 0,-5.5714829361,-3.8773853389,1.8284586655$ $\mathrm{H}, 0,-6.2100514501,-3.9374023611,0.1701870336$ C,0,-3.8559979705,-3.4411288493,-1.1589465613 $\mathrm{H}, 0,-4.73788666,-3.3008178652,-1.7960262501$ H,0,-3.0461568151,-2.825599257,-1.5520961979 $\mathrm{H}, 0,-3.5570761956,-4.4947360809,-1.2273924016$ C, $0,1.4942516399,-3.3811388195,-0.215623495$ C, $0,1.0244707325,-2.9115360964,1.1832698322$ $\mathrm{H}, 0,0.2936704603,-2.1048144246,1.1086963077$ $\mathrm{H}, 0,0.5636351286,-3.7470136217,1.7249608905$ $\mathrm{H}, 0,1.8766359667,-2.5567170407,1.7763325212$ C, $0,0.3018778316,-3.9604814565,-1.0147113933$ $\mathrm{H}, 0,-0.4824320161,-3.216878605,-1.1580733009$ $\mathrm{H}, 0,0.6333027484,-4.3138612924,-1.9989423971$ $\mathrm{H}, 0,-0.1263652147,-4.8162622324,-0.4778147683$ C,0,2.5016087858,-4.5292736585,0.0011531419 $\mathrm{H}, 0,2.0063989827,-5.3383235994,0.5497508907$ $\mathrm{H}, 0,2.8629969158,-4.9478316962,-0.9455582814$ $\mathrm{H}, 0,3.3692375177,-4.214521841,0.592823538$ C, $0,-7.724632284,0.362687053,1.5966444849$ C, $0,-7.8582877204,-0.2564014117,3.0087044238$ $\mathrm{H}, 0,-8.8651387757,-0.0812831943,3.4070625478$ $\mathrm{H}, 0,-7.689563113,-1.3385950539,2.999349451$ $\mathrm{H}, 0,-7.1359453501,0.1901576492,3.7019816686$ C,0,-8.0336433397,1.8681799263,1.7025693212 $\mathrm{H}, 0,-7.3368867709,2.3803659552,2.3763236132$ $\mathrm{H}, 0,-7.9918495268,2.3627299253,0.7251976835$ $\mathrm{H}, 0,-9.0443312375,2.0093602465,2.1016380016$ C, $0,-8.772614547,-0.2790577361,0.6557736176$ $\mathrm{H}, 0,-8.7125853155,0.1550468447,-0.3490150451$ $\mathrm{H}, 0,-8.6301759448,-1.3611094153,0.5626708397$ H,0,-9.7858142587,-0.1087159078,1.0403452726 
C, $0,5.5897353677,-1.3295538384,-2.4674234007$

C, $0,6.4512355439,-1.5775666601,-1.2058745562$

$\mathrm{H}, 0,6.1425793824,-2.4819854122,-0.6707442663$

$\mathrm{H}, 0,7.5052274284,-1.699791832,-1.4838906697$

$\mathrm{H}, 0,6.3788806449,-0.7339057129,-0.5095243185$

C,0,6.1337060314,-0.0774395082,-3.1813622165

$\mathrm{H}, 0,5.5830181797,0.1360905447,-4.104713386$

$\mathrm{H}, 0,6.0855477982,0.8103918956,-2.5400932855$

$\mathrm{H}, 0,7.1841993685,-0.2337853695,-3.451284517$

C, $0,5.7341707456,-2.5338857832,-3.4287467429$

$\mathrm{H}, 0,5.3899967123,-3.4665729805,-2.9688109973$

$\mathrm{H}, 0,5.1511641733,-2.375446612,-4.3434040487$

H,0,6.7846222057,-2.6705789609,-3.7137170209

$\mathrm{Cl}, 0,-2.0902438119,-0.253131665,-3.5143801165$

$\mathrm{HF}=-2125.5480947$ (A.U.)

Zero-point correction $=0.820929$ (A.U.)

Total Gibbs free energy $=-2124.802268$ (A.U.)

$(R, R)-\mathbf{1} / \mathbf{2 a} / \mathrm{Cl}-1$

Cartesian Coordinates (Angstroms)

C,0,3.9727619769,-3.217944154,-2.089930751

C, $0,2.7618467029,-2.5381338928,-2.1501684418$

C, $0,1.8949880034,-2.597214339,-1.0035229864$

C, $0,2.3048186743,-3.4180671668,0.0948182401$

C, $0,3.5551159407,-4.080335263,0.0814981241$

C, $0,4.4192820896,-3.9916411933,-0.9913552491$

$\mathrm{H}, 0,4.6254255826,-3.1641135704,-2.9532196835$

$\mathrm{H}, 0,3.8114952802,-4.677833078,0.9517491474$

C,0,1.4893370386,-3.6308813416,1.2624420959

$\mathrm{N}, 0,0.2820658913,-3.2057774385,1.4417125919$

$\mathrm{N}, 0,-2.2753796716,-2.5450949848,1.3588785122$

C, $0,-3.3921140488,-1.9006839244,1.3904873916$

C, $0,-3.8122940375,-0.8790316156,0.4611487265$

C, $0,-3.0637952031,-0.5411142385,-0.7133719671$

C, $0,-5.0649479189,-0.2746785364,0.7204514485$

C, $0,-3.6776884889,0.3584744571,-1.6584349128$

C, $0,-5.6279723512,0.6495263413,-0.1381802921$

$\mathrm{H}, 0,-5.5814039188,-0.5724042304,1.6287278235$

C, $0,-4.9033582503,0.9222015531,-1.3247164633$

$\mathrm{H}, 0,-5.3529154789,1.6096396472,-2.0318352566$

$\mathrm{O}, 0,-1.8764220606,-1.0300496754,-0.9505095195$

O,0,0.7809009186,-1.9047171758,-0.970969439

Al, $0,-0.7692474812,-2.1659635887,0.0282463765$

$\mathrm{H}, 0,1.9653752296,-4.2241869578,2.0474829128$

$\mathrm{H}, 0,-4.125387277,-2.1426625734,2.1648393438$ 
$\mathrm{P}, 0,1.098224647,2.9951679638,0.5547569199$ $\mathrm{C}, 0,-0.5676099814,1.2191738337,2.7448607566$ $\mathrm{H}, 0,-0.070229127,0.7109470551,3.579076443$ $\mathrm{H}, 0,-1.6413800442,1.0290491922,2.8429552786$ $\mathrm{H}, 0,-0.3883205646,2.2920730579,2.8435303365$ $\mathrm{O}, 0,-0.4010585202,-0.6187465119,1.3019715453$ C, $0,-0.0881025129,0.6035687506,1.436114518$ C, $0,0.651281262,1.3196293949,0.4854122289$ $\mathrm{H}, 0,0.9702901894,0.7938887277,-0.4081723478$ C, $0,-1.9517019328,-3.6389203362,2.2825083362$ C, $0,-0.4651349914,-3.4473241459,2.6848600389$ C, $0,-2.8466461831,-3.7997213238,3.5168529557$ C, $0,0.0207117749,-4.6044118158,3.5673456274$ $\mathrm{H}, 0,-0.411156092,-2.5107139582,3.2601234813$ C, $0,-2.3534288212,-4.9582319812,4.3988711041$ $\mathrm{H}, 0,-2.8465725948,-2.8657607911,4.0985350155$ C, $0,-0.8838967349,-4.7739746352,4.7982998236$ $\mathrm{H}, 0,1.0507863298,-4.4293983724,3.9004641167$ H, $0,0.0268234531,-5.5308702637,2.9763008195$ H,0,-2.9841115955,-5.0386845949,5.2928254419 $\mathrm{H}, 0,-2.4658697045,-5.9035366658,3.8496486448$ $\mathrm{H}, 0,-0.5408285404,-5.6283608135,5.3945169521$ $\mathrm{H}, 0,-0.7912766809,-3.8863961672,5.4413474035$ $\mathrm{H}, 0,-2.0043902554,-4.555225694,1.6777457317$ $\mathrm{H}, 0,-3.8830426595,-3.988355354,3.2121940293$ C, $0,-3.0141757286,0.6395022516,-3.023605848$ C, $0,-2.8511326185,-0.6923788642,-3.7979761202$ $\mathrm{H}, 0,-3.8302748569,-1.1484938408,-3.9871533468$ $\mathrm{H}, 0,-2.37394809,-0.5044482237,-4.7685811188$ $\mathrm{H}, 0,-2.2420073334,-1.4078380842,-3.2442776765$ C, $0,-3.8584890619,1.5842856523,-3.9034674634$ $\mathrm{H}, 0,-3.3467645628,1.7349375617,-4.8610674927$ $\mathrm{H}, 0,-4.8490435183,1.169752173,-4.1244501899$ $\mathrm{H}, 0,-3.9925871889,2.5721229048,-3.445048308$ C, $0,-1.6312600742,1.3054570812,-2.831972912$ $\mathrm{H}, 0,-1.7401485134,2.2801441477,-2.3379163347$ $\mathrm{H}, 0,-0.9756077532,0.6763749376,-2.229988724$ $\mathrm{H}, 0,-1.1589382372,1.4710807129,-3.8095143773$ C, $0,2.3368437666,-1.7847463927,-3.4281041724$ C, $0,1.0339257995,-2.4097718702,-3.9843388628$ $\mathrm{H}, 0,0.2336624746,-2.402523359,-3.2434053042$ H,0,0.6992858664,-1.8526501082,-4.869545994 $\mathrm{H}, 0,1.2046667669,-3.4501988588,-4.2842125207$ C,0,2.1141397626,-0.2858753293,-3.1229736053 $\mathrm{H}, 0,1.3080014372,-0.1545685087,-2.4012485661$ $\mathrm{H}, 0,3.0268081606,0.1720743648,-2.7216692642$ H, $0,1.845673722,0.2494943297,-4.0434586016$ 
C,0,3.4020964204,-1.8670563434,-4.5411621853 $\mathrm{H}, 0,3.0390949772,-1.3258234122,-5.4226079048$ $\mathrm{H}, 0,4.3535856508,-1.4093160219,-4.2427210974$ $\mathrm{H}, 0,3.5985813906,-2.8999591582,-4.8512396926$ C, $0,-6.9797656834,1.3388382524,0.1139424064$ C, $0,-7.9720963014,0.9793348704,-1.0181394593$ $\mathrm{H}, 0,-8.9404637357,1.4693061835,-0.8538467342$ $\mathrm{H}, 0,-7.6024389529,1.2948447027,-1.9995812317$ $\mathrm{H}, 0,-8.1396646429,-0.1028512397,-1.0584962705$ C, $0,-7.6098671467,0.9105370727,1.4531462562$ $\mathrm{H}, 0,-7.8148193528,-0.1657871275,1.4795670255$ $\mathrm{H}, 0,-6.963597711,1.1561506818,2.3041640465$ $\mathrm{H}, 0,-8.5631373497,1.431733672,1.5995423386$ C, $0,-6.784306645,2.8737797453,0.1482412463$ H, $0,-6.1078411918,3.1608777089,0.9629472345$ $\mathrm{H}, 0,-6.361350016,3.2488865025,-0.7902480532$ $\mathrm{H}, 0,-7.7425647703,3.3844438917,0.3081786136$ C, $0,5.7864362876,-4.6939527283,-1.0497102147$ C, $0,5.813136674,-5.6896049305,-2.2342853449$ H,0,5.6359124702,-5.1881909066,-3.1916257621 $\mathrm{H}, 0,6.7880384074,-6.1902739266,-2.2957042115$ $\mathrm{H}, 0,5.041222402,-6.4581515706,-2.1142240115$ C, $0,6.089622625,-5.4809942858,0.23959716$ $\mathrm{H}, 0,6.1094015748,-4.8277007055,1.1200078708$ $\mathrm{H}, 0,5.3505630735,-6.2708768435,0.4162719273$ $\mathrm{H}, 0,7.0725782158,-5.9598690685,0.1603819327$ C, $0,6.9059092471,-3.6430906533,-1.2424537407$ $\mathrm{H}, 0,6.7684563251,-3.0670724829,-2.1637669985$ $\mathrm{H}, 0,6.9242127401,-2.9342153452,-0.4061828921$ $\mathrm{H}, 0,7.8877216107,-4.1306365885,-1.2975753193$ C, $0,2.2618758508,3.3428932381,-0.8075405487$ C,0,3.5999411188,3.6851271918,-0.5691898367 C, $0,1.7894835902,3.257682265,-2.1279795324$ C, $0,4.4580459047,3.9343898065,-1.6419596636$ $\mathrm{H}, 0,3.9746098324,3.7558451031,0.4463647726$ C,0,2.6532382554,3.5054161147,-3.1935784186 H, $0,0.7544313274,2.9950500874,-2.3252276152$ C, $0,3.9869960976,3.8443728549,-2.9521947599$ $\mathrm{H}, 0,5.494366577,4.1978791551,-1.4504179079$ H,0,2.2836962398,3.4298914244,-4.2118819744 $\mathrm{H}, 0,4.6573808022,4.0368225417,-3.785195876$ C, $0,1.9386449827,3.4420139137,2.1250185744$ C, $0,1.7304796957,4.6594939619,2.7890663972$ C, $0,2.8375337569,2.5065950789,2.6663457643$ C, $0,2.4190680657,4.9402469217,3.9708060915$ $\mathrm{H}, 0,1.0229576101,5.3828787384,2.3989119457$ C,0,3.5288603008,2.7962807381,3.842280525 
$\mathrm{H}, 0,2.9795344316,1.5495809335,2.1724326645$

C, $0,3.3206986419,4.0133148829,4.4960840663$

$\mathrm{H}, 0,2.2450588685,5.8828245323,4.4821689134$

$\mathrm{H}, 0,4.2223089431,2.0672257497,4.2517639393$

$\mathrm{H}, 0,3.8540169899,4.234640514,5.4164372348$

C, $0,-0.2865713273,4.175691574,0.3098658653$

C, $0,-0.042592204,5.5339527447,0.0402230289$

C, $0,-1.6058222382,3.703618486,0.3362940268$

C, $0,-1.1083705106,6.409024134,-0.164766531$

$\mathrm{H}, 0,0.9763772354,5.9053549842,-0.0287148416$

C, $0,-2.6701254506,4.5837738319,0.1270865921$

$\mathrm{H}, 0,-1.7998995079,2.6469799093,0.4890424691$

C, $0,-2.4229140609,5.9352068373,-0.1163634181$

$\mathrm{H}, 0,-0.913000377,7.4570023787,-0.3744725712$

$\mathrm{H}, 0,-3.6874865769,4.2037678209,0.1403873379$

$\mathrm{H}, 0,-3.2513222455,6.6179835708,-0.284003473$

$\mathrm{Cl}, 0,-1.3592094832,-4.1560159733,-1.1073701552$

$\mathrm{HF}=-3353.747814$ (A.U.)

Zero-point correction $=1.162805$ (A.U.)

Total Gibbs free energy $=-3352.689146$ (A.U.)

$(R, R)-\mathbf{1} / \mathbf{2 a} / \mathrm{Cl}-1$ (optimized in the solvent )

Cartesian Coordinates (Angstroms)

C,0,3.9382066721,-3.2464663673,-2.102066202

C, $0,2.7378721788,-2.5439225814,-2.1514766168$

C, $0,1.8572459136,-2.6304946447,-1.017084771$

C, $0,2.2590978287,-3.4663853038,0.0721585858$

C,0,3.4989884638,-4.1486660205,0.0491441304

C, $0,4.3658529106,-4.0558076813,-1.0224855333$

$\mathrm{H}, 0,4.5999040215,-3.1753720578,-2.9568829797$

H, 0,3.7474620081,-4.7583199245,0.9126381152

C, $0,1.4595236811,-3.6515820766,1.2579201056$

$\mathrm{N}, 0,0.2739335987,-3.1777058867,1.4584751264$

$\mathrm{N}, 0,-2.2754282628,-2.5141455045,1.3908875304$

C, $0,-3.4207208817,-1.9177904063,1.3823063568$

$\mathrm{C}, 0,-3.866778887,-0.9418688624,0.4163050951$

C, $0,-3.1067065105,-0.5840738635,-0.7435711033$

C, $0,-5.1542665984,-0.3972630789,0.6385349201$

C, $0,-3.7283340194,0.2914051901,-1.7035778049$

C, $0,-5.7384139599,0.4904734456,-0.2443642068$

$\mathrm{H}, 0,-5.6769580569,-0.7091831182,1.5377507426$

C, $0,-4.9913943607,0.7949309438,-1.4077444845$

$\mathrm{H}, 0,-5.4484181151,1.4657546973,-2.1251928874$

O,0,-1.8933972399,-1.0409797023,-0.9502089961

$\mathrm{O}, 0,0.7339284748,-1.9461239692,-0.9875485725$ 
$\mathrm{Al}, 0,-0.7643520758,-2.0780119241,0.1033093787$

$\mathrm{H}, 0,1.9296741602,-4.252162759,2.0390229548$

$\mathrm{H}, 0,-4.1558006083,-2.1704924519,2.1491834503$

$\mathrm{P}, 0,1.1527501146,3.0721678193,0.5772306984$

C, $0,-0.4836796209,1.2462299333,2.7806682563$

$\mathrm{H}, 0,0.0360649375,0.7142407036,3.5859306652$

$\mathrm{H}, 0,-1.5557063738,1.0622086986,2.9089699361$

$\mathrm{H}, 0,-0.294961761,2.3147601063,2.8987303597$

$\mathrm{O}, 0,-0.3688789937,-0.5634404941,1.2920424497$

C, $0,-0.0496091311,0.6689721793,1.443971893$

C, $0,0.6479418459,1.3997996706,0.4871044162$

$\mathrm{H}, 0,0.9241406083,0.9004556282,-0.4350469135$

C, $0,-1.9387987384,-3.5706595689,2.3600719745$

$\mathrm{C}, 0,-0.4482068173,-3.3675266162,2.7284920662$

C, $0,-2.8151384169,-3.6714161479,3.6143190457$

C, $0,0.0562165146,-4.4792427162,3.6552787453$

$\mathrm{H}, 0,-0.3784654296,-2.4062672483,3.2577795559$

C, $0,-2.3029037997,-4.7818522232,4.5467515348$

$\mathrm{H}, 0,-2.8103915207,-2.708038201,4.1442986518$

C, $0,-0.8268282292,-4.5802505719,4.9098283532$

$\mathrm{H}, 0,1.0917827209,-4.284918342,3.9579055233$

$\mathrm{H}, 0,0.050650259,-5.4372702254,3.1166183632$

$\mathrm{H}, 0,-2.9177993339,-4.8107125009,5.4540855767$

$\mathrm{H}, 0,-2.4265363001,-5.7552695116,4.0517585741$

$\mathrm{H}, 0,-0.4740494001,-5.4044651417,5.5409549189$

$\mathrm{H}, 0,-0.7192097552,-3.6613413718,5.5035741673$

$\mathrm{H}, 0,-2.0053682032,-4.5139248386,1.8003410602$

$\mathrm{H}, 0,-3.8544810348,-3.8823288355,3.3375091351$

C, $0,-3.0323367207,0.6396217555,-3.0381052677$

C, $0,-2.7620829965,-0.6588790826,-3.8370379709$

$\mathrm{H}, 0,-3.7044772569,-1.1675840903,-4.0747317518$

$\mathrm{H}, 0,-2.261638308,-0.4192939654,-4.7841159353$

$\mathrm{H}, 0,-2.1306986425,-1.3482103657,-3.2754931406$

C, $0,-3.8969583764,1.552528982,-3.9327610898$

$\mathrm{H}, 0,-3.3580921935,1.750614916,-4.8664947036$

$\mathrm{H}, 0,-4.8537939891,1.0881084779,-4.1984100442$

$\mathrm{H}, 0,-4.1036545261,2.5213020197,-3.4623695516$

C, $0,-1.7025612089,1.3852422314,-2.7778582206$

$\mathrm{H}, 0,-1.8888017126,2.3310174215,-2.2534717834$

$\mathrm{H}, 0,-1.0222664697,0.7837143523,-2.1750800421$

$\mathrm{H}, 0,-1.2110793345,1.618831705,-3.7313958385$

C, $0,2.3573590094,-1.7206635768,-3.401410707$

C,0,1.0539004213,-2.2842893224,-4.015988717

$\mathrm{H}, 0,0.2379467676,-2.2709592586,-3.2925692604$

$\mathrm{H}, 0,0.7593328548,-1.6865372367,-4.8884010297$

H,0,1.2017539112,-3.3182441378,-4.3507519636

C, $0,2.1603359047,-0.2336123891,-3.0268331128$ 
$\mathrm{H}, 0,1.3473199941,-0.1182518476,-2.3101281856$ $\mathrm{H}, 0,3.0750419115,0.1848831017,-2.5892852687$ H,0,1.9193877909,0.3539164281,-3.9217921344 C, $0,3.4433723599,-1.7724005185,-4.4964487697$ $\mathrm{H}, 0,3.1109587929,-1.1768139998,-5.3544055854$ $\mathrm{H}, 0,4.3979046679,-1.3535054921,-4.1556678856$ $\mathrm{H}, 0,3.6236808546,-2.7922388511,-4.8558892116$ C, $0,-7.1262870091,1.1193355033,-0.0314762354$ C, $0,-8.0668493797,0.7200486808,-1.1938974356$ $\mathrm{H}, 0,-9.0582290682,1.1698367287,-1.0567404553$ $\mathrm{H}, 0,-7.6821628791,1.0540744273,-2.1635278461$ $\mathrm{H}, 0,-8.1903428151,-0.3682327149,-1.2390912353$ C, $0,-7.7751699528,0.6581262715,1.2877461975$ $\mathrm{H}, 0,-7.9293031784,-0.4269312045,1.3081763413$ $\mathrm{H}, 0,-7.1683989394,0.9335929858,2.1583336301$ $\mathrm{H}, 0,-8.7557388217,1.1339090331,1.4034293856$ C,0,-6.9963611216,2.6610158824,0.0108738207 $\mathrm{H}, 0,-6.3538974287,2.9754902811,0.8423593475$ $\mathrm{H}, 0,-6.5664049276,3.0581663818,-0.9150236089$ H,0,-7.9806090727,3.1264027307,0.1473125842 C, $0,5.7240493459,-4.7755054529,-1.0900590835$ C, $0,5.7433285934,-5.7426764755,-2.2981970059$ $\mathrm{H}, 0,5.5753885467,-5.216676295,-3.244145414$ $\mathrm{H}, 0,6.7138853589,-6.2500299251,-2.3668077018$ $\mathrm{H}, 0,4.9651649645,-6.5082017799,-2.1977803254$ C,0,6.0087011406,-5.5956760594,0.1827495246 $\mathrm{H}, 0,6.0343032245,-4.9630507324,1.0777791154$ $\mathrm{H}, 0,5.2575942254,-6.3785968418,0.3383977531$ $\mathrm{H}, 0,6.9851425438,-6.0859172207,0.0956455431$ C, $0,6.8578922189,-3.7349484678,-1.2537445221$ $\mathrm{H}, 0,6.7351344113,-3.1377685377,-2.1637284243$ $\mathrm{H}, 0,6.8823813729,-3.0456334854,-0.4013755271$ H, $0,7.831918762,-4.2364953402,-1.3135608957$ C, $0,2.2888538191,3.3831967343,-0.8169395101$ C,0,3.6698610997,3.5238991562,-0.6205057293 C,0,1.7585976173,3.4582061644,-2.1163184092 C, $0,4.5117979783,3.7343009605,-1.7146056476$ $\mathrm{H}, 0,4.0909840506,3.472458109,0.3777155647$ C,0,2.6059036604,3.6669833752,-3.2039149973 $\mathrm{H}, 0,0.6899538416,3.359744548,-2.2814024861$ C, $0,3.982359468,3.8058959628,-3.0042671593$ $\mathrm{H}, 0,5.5803099082,3.8446673798,-1.5552126864$ H,0,2.1900842496,3.7241887652,-4.205416206 $\mathrm{H}, 0,4.6396614773,3.9718951155,-3.8529202059$ C, $0,2.0383483578,3.453159318,2.1327038162$ C, $0,1.8916014866,4.6761813205,2.8046331787$ C,0,2.9097745212,2.4785320749,2.6504992926 
C,0,2.6144360088,4.9221366076,3.9735670704

$\mathrm{H}, 0,1.2099454477,5.4322725009,2.4313959483$

C,0,3.6341244954,2.7341844222,3.8145885474

$\mathrm{H}, 0,3.0113531299,1.5201109537,2.1503395665$

C, $0,3.4873373997,3.9557657037,4.4772306303$

$\mathrm{H}, 0,2.4900678889,5.8689089513,4.4907594749$

$\mathrm{H}, 0,4.3061138282,1.9764648001,4.2064967909$

$\mathrm{H}, 0,4.0472340423,4.1509671264,5.3872876553$

C, $0,-0.2092430559,4.28044987,0.3804741642$

C, $0,0.0619037625,5.6311207243,0.0968175538$

C, $0,-1.538872891,3.8482645963,0.4825944962$

C, $0,-0.9879233031,6.5364167465,-0.0554295405$

$\mathrm{H}, 0,1.0853346903,5.9762849196,-0.019023147$

$\mathrm{C}, 0,-2.5860610701,4.759215799,0.327299269$

$\mathrm{H}, 0,-1.7548074656,2.8001831654,0.6607299586$

C, $0,-2.3117603294,6.1023091076,0.0634409289$

$\mathrm{H}, 0,-0.7723232497,7.5778673285,-0.275098518$

$\mathrm{H}, 0,-3.6129842557,4.4142251798,0.4035601579$

$\mathrm{H}, 0,-3.1268883778,6.8093603906,-0.0608271705$

$\mathrm{Cl}, 0,-1.449891118,-4.2134035036,-1.0842281579$

$\mathrm{HF}=-3353.7802601$ (A.U.)

Zero-point correction $=1.162548$ (A.U.)

Total Gibbs free energy $=-3352.720626$ (A.U.)

$(R, R)-\mathbf{1} / \mathbf{2 a} / \mathrm{Cl}-2$

Cartesian Coordinates (Angstroms)

C, $0,4.8955646773,2.3190562325,-0.4870436546$

C, $0,3.8215338081,1.5926866455,-0.9942145771$

C, $0,2.9085561848,1.0139395361,-0.0551412283$

C,0,3.178473384,1.1748717699,1.3347340997

C, $0,4.2990350213,1.9144532987,1.776668731$

C, $0,5.1703687322,2.5134847512,0.8867245554$

$\mathrm{H}, 0,5.5784923932,2.7704026905,-1.1962676826$

$\mathrm{H}, 0,4.4528219815,1.9978680591,2.8484004016$

C, $0,2.3832715488,0.5226639123,2.3436520006$

$\mathrm{N}, 0,1.2840440385,-0.1235991509,2.1286352379$

$\mathrm{N}, 0,-0.3387920478,-1.9013546844,1.179452313$

C, $0,-1.3318487232,-2.5953377932,0.7058921675$

C, $0,-1.9519806923,-2.4302720719,-0.5763766456$

C, $0,-1.4405771187,-1.5169890084,-1.5473106902$

C, $0,-3.0452459098,-3.2799570255,-0.8761974403$

$\mathrm{C}, 0,-2.0171082478,-1.536513769,-2.8621754494$

C,0,-3.6477014004,-3.2710078674,-2.1170891049

$\mathrm{H}, 0,-3.3937083585,-3.9422238255,-0.0896932636$

C,0,-3.0885164106,-2.3948518171,-3.0812745586 
$\mathrm{H}, 0,-3.534497298,-2.4010426259,-4.0686576213$ $\mathrm{O}, 0,-0.4545498816,-0.6997252664,-1.2512621003$ $\mathrm{O}, 0,1.8587739906,0.3254032979,-0.4801733857$ Al, $0,0.3226923698,-0.1974048151,0.3488677991$ $\mathrm{H}, 0,2.7786598558,0.5693297721,3.3606665435$ $\mathrm{H}, 0,-1.7321649645,-3.4057185335,1.3149713934$ $\mathrm{P}, 0,-4.6409645429,1.805424791,0.8481727964$ C, $0,-1.7188548136,3.3628293429,1.0253627897$ $\mathrm{H}, 0,-2.6740115102,3.8880685811,0.9873335482$ $\mathrm{H}, 0,-1.0424594515,3.7895840297,0.2769892758$ $\mathrm{H}, 0,-1.2671745079,3.5358892313,2.0086852703$ $\mathrm{O}, 0,-0.6571484651,1.2965116603,0.7349034906$ C, $0,-1.8214251345,1.8728887793,0.7935427222$ C, $0,-3.0058633316,1.181706706,0.6679682785$ $\mathrm{H}, 0,-2.9715649348,0.1064058555,0.526770611$ C, $0,0.2751645401,-2.2474390949,2.4860862159$ C, $0,0.5922054121,-0.898172561,3.1727906691$ C, $0,-0.515912185,-3.183232653,3.4038481913$ C, $0,1.3314751993,-1.1022186274,4.4972284789$ $\mathrm{H}, 0,-0.3738047833,-0.4109715722,3.3792972494$ C, $0,0.2308536652,-3.3741636592,4.7359610968$ H, $0,-1.5007663935,-2.7406516595,3.600731929$ C, $0,0.5192170504,-2.0293142103,5.417098297$ H,0,1.4864028706,-0.1355049727,4.9928996297 $\mathrm{H}, 0,2.3268351392,-1.535120142,4.3089887275$ $\mathrm{H}, 0,-0.3734145793,-4.0068181223,5.396698241$ $\mathrm{H}, 0,1.1747695506,-3.9133598259,4.5599708703$ $\mathrm{H}, 0,1.0680354166,-2.1882623635,6.3538534772$ $\mathrm{H}, 0,-0.4320163098,-1.5417275947,5.6615904082$ $\mathrm{H}, 0,1.24166939,-2.7185942775,2.2416263251$ H,0,-0.6526913279,-4.162170901,2.9252220685 C, $0,-1.4584859351,-0.6475928132,-3.9917295687$ C, $0,0.0304967222,-0.9935866537,-4.2341355683$ $\mathrm{H}, 0,0.137572359,-2.0412389953,-4.5410275757$ $\mathrm{H}, 0,0.4363520739,-0.3656210398,-5.0373954097$ $\mathrm{H}, 0,0.6274335934,-0.8323038165,-3.3362200931$ C, $0,-2.2024657326,-0.8574965939,-5.3265266025$ $\mathrm{H}, 0,-1.7562713882,-0.2111378799,-6.09094252$ $\mathrm{H}, 0,-2.1239696903,-1.8905147865,-5.6852856599$ $\mathrm{H}, 0,-3.2654682259,-0.5966721646,-5.257149771$ C, $0,-1.601962703,0.8461676041,-3.6134047711$ $\mathrm{H}, 0,-2.6612754228,1.110089058,-3.5051114246$ $\mathrm{H}, 0,-1.0884602989,1.0686560534,-2.6767477045$ $\mathrm{H}, 0,-1.174963614,1.4781783098,-4.4024114223$ C, $0,3.6204474146,1.420964914,-2.514277854$ C, $0,3.6636309667,-0.0831583497,-2.8788625987$ $\mathrm{H}, 0,2.8929348544,-0.6441970585,-2.3487722383$ 
$\mathrm{H}, 0,3.5078946562,-0.2137989778,-3.9571392951$ $\mathrm{H}, 0,4.6412352371,-0.5120887094,-2.6262996308$ C,0,2.2659713932,2.0366861786,-2.9408940125 $\mathrm{H}, 0,1.431952082,1.5521405964,-2.4325382513$ H,0,2.2405957468,3.1092262644,-2.7109298037 $\mathrm{H}, 0,2.125677685,1.9220305096,-4.0232450969$ C, $0,4.722512563,2.1223318486,-3.3349518363$ H,0,4.5306781792,1.9613065033,-4.4018575915 $\mathrm{H}, 0,4.7407788078,3.205587059,-3.1657010919$ H,0,5.7201189519,1.7223054116,-3.118048426 C, $0,-4.8476198121,-4.1573351804,-2.4909373321$ C, $0,-4.4540893687,-5.1076498213,-3.6469364682$ $\mathrm{H}, 0,-5.305661385,-5.7377890053,-3.9326250427$ $\mathrm{H}, 0,-4.1358427622,-4.5560880501,-4.5382983947$ $\mathrm{H}, 0,-3.6288606578,-5.7648731183,-3.3495492174$ C, $0,-5.3234186961,-5.0146154928,-1.3025290715$ $\mathrm{H}, 0,-4.5416422192,-5.6999121951,-0.9549588411$ $\mathrm{H}, 0,-5.6380956164,-4.3935370379,-0.4559158911$ $\mathrm{H}, 0,-6.1826379688,-5.6237581563,-1.6060349152$ C, $0,-6.0307354043,-3.2668510063,-2.9406378476$ $\mathrm{H}, 0,-6.3396602167,-2.5944277617,-2.1323267478$ $\mathrm{H}, 0,-5.7725429971,-2.6511447648,-3.8090723212$ $\mathrm{H}, 0,-6.8918781293,-3.8875820449,-3.2189114307$ C, $0,6.3921420507,3.3442556873,1.3162174593$ C,0,7.6851436457,2.6865372181,0.7774941739 $\mathrm{H}, 0,7.6811364179,2.6140069755,-0.3153556854$ $\mathrm{H}, 0,8.5648966466,3.27398465,1.0686932807$ $\mathrm{H}, 0,7.8044168917,1.6734784007,1.1786296501$ C, $0,6.5109734121,3.4494893541,2.8484854279$ $\mathrm{H}, 0,5.6308549966,3.9289368847,3.2926043706$ $\mathrm{H}, 0,6.6379418842,2.4662191448,3.3162656602$ H,0,7.3856972118,4.0549569193,3.1118528107 C, $0,6.2721870442,4.7776211529,0.7453483913$ $\mathrm{H}, 0,6.2224728129,4.7786775411,-0.3488283396$ $\mathrm{H}, 0,5.369931069,5.273713717,1.1211002355$ $\mathrm{H}, 0,7.140304004,5.3811142567,1.0387269057$ C, $0,-5.2112448232,2.7324725754,-0.6264691784$ C, $0,-6.523508205,3.2313958115,-0.7311407584$ C, $0,-4.314281077,2.9424273783,-1.6822529015$ C,0,-6.9205192021,3.9305094032,-1.8704799965 $\mathrm{H}, 0,-7.2384735854,3.0705880426,0.0705459226$ C,0,-4.7157694794,3.6458076992,-2.8200469125 $\mathrm{H}, 0,-3.3075826186,2.5437889248,-1.6156060601$ C,0,-6.0162359114,4.1408690956,-2.915440533 $\mathrm{H}, 0,-7.9360812196,4.3095722279,-1.9420253146$ $\mathrm{H}, 0,-4.0098575167,3.800041899,-3.6309853439$ $\mathrm{H}, 0,-6.3284816921,4.6862255153,-3.8017399802$ 
C, $0,-4.815330654,2.9089002882,2.3009143101$

C, $0,-4.2841690352,2.4617618809,3.5231257181$

C, $0,-5.4393388206,4.1656655457,2.2285058695$

C, $0,-4.4062608215,3.2615122615,4.6601692494$

$\mathrm{H}, 0,-3.7622969564,1.5065830942,3.618335791$

C, $0,-5.5477251745,4.9575255862,3.3720394101$

$\mathrm{H}, 0,-5.8248159829,4.5425337377,1.2882724564$

C, $0,-5.0374715148,4.5044230439,4.5903924986$

$\mathrm{H}, 0,-3.995640592,2.8970482701,5.5972416006$

$\mathrm{H}, 0,-6.0276139866,5.930230524,3.3060548866$

$\mathrm{H}, 0,-5.1254122834,5.123629406,5.4793691391$

C, $0,-5.7188776072,0.3407901622,1.0414208437$

C, $0,-6.7678767474,0.0644802482,0.1499032603$

C, $0,-5.4549388344,-0.5382204668,2.1063977421$

C,0,-7.5608012843,-1.0689899278,0.3354949215

$\mathrm{H}, 0,-6.9658583562,0.7160111678,-0.6932558002$

C, $0,-6.2554405183,-1.668242051,2.2791105443$

$\mathrm{H}, 0,-4.622819824,-0.3854844294,2.7972926546$

C, $0,-7.3099983456,-1.9333771114,1.4035702042$

$\mathrm{H}, 0,-8.3728275606,-1.2737461136,-0.3568479585$

$\mathrm{H}, 0,-6.0394041547,-2.3365060992,3.1076501244$

$\mathrm{H}, 0,-7.9330895017,-2.8122343673,1.5482306724$

$\mathrm{Cl}, 0,-2.7747371383,-0.5404944141,4.3545404191$

$\mathrm{HF}=-3353.7521243$ (A.U.)

Zero-point correction $=1.164167$ (A.U.)

Total Gibbs free energy $=-3352.690550$ (A.U.)

$(R, R)-\mathbf{1} / \mathbf{2 a} / \mathrm{Cl}-2$ (optimized in the solvent)

Cartesian Coordinates (Angstroms)

C, $0,4.9295593917,2.2639411418,-0.5175740833$

C, $0,3.8161283026,1.5921369581,-1.0178027989$

C, $0,2.9087134438,1.0150120479,-0.0720061068$

C, $0,3.2184274436,1.1310482363,1.3140606297$

C, $0,4.3771642786,1.8156852948,1.7491266247$

C, $0,5.2483930826,2.406479887,0.853362793$

$\mathrm{H}, 0,5.6095227407,2.7122213969,-1.2312930274$

$\mathrm{H}, 0,4.5586724198,1.8630086487,2.8182917807$

C, $0,2.4167946516,0.4996680464,2.32887293$

$\mathrm{N}, 0,1.2864896542,-0.0977520824,2.1257705716$

$\mathrm{N}, 0,-0.4042247306,-1.8341066734,1.211242547$

C, $0,-1.3420023164,-2.5779536662,0.69693316$

C, $0,-1.932916687,-2.4363736764,-0.6002608908$

C, $0,-1.4498673079,-1.4881258053,-1.5519812977$

C, $0,-2.9753962272,-3.3396371019,-0.9288385641$

C, $0,-2.01898608,-1.5036522146,-2.8698963112$ 
C, $0,-3.5602128964,-3.3416053696,-2.1782323092$ $\mathrm{H}, 0,-3.2967894632,-4.0336878507,-0.1586662864$ C, $0,-3.0426183371,-2.4129789874,-3.1164191166$ $\mathrm{H}, 0,-3.482160124,-2.419222647,-4.1064159368$ O,0,-0.4918415521,-0.6429858472,-1.2336329929 $\mathrm{O}, 0,1.8253773064,0.3696783359,-0.4849734942$ Al, $0,0.2938586349,-0.1510015771,0.3628765157$ $\mathrm{H}, 0,2.8290585216,0.525418689,3.3386348245$ $\mathrm{H}, 0,-1.7188500325,-3.4140649354,1.2853062327$ $\mathrm{P}, 0,-4.6677736782,1.8249273105,0.8820853383$ C, $0,-1.7563482062,3.4143108026,1.0032141431$ $\mathrm{H}, 0,-2.7209705495,3.9225009688,0.9877401571$ $\mathrm{H}, 0,-1.1257552532,3.8256429225,0.2074536133$ $\mathrm{H}, 0,-1.2619148539,3.6342639874,1.9560897679$ $\mathrm{O}, 0,-0.6706842195,1.3470549823,0.7812552198$ C, $0,-1.844268379,1.9164539685,0.8278894226$ C, $0,-3.0205910327,1.2063533436,0.7428516656$ $\mathrm{H}, 0,-2.9749840286,0.1300659963,0.6084772729$ C, $0,0.1825880284,-2.1740683014,2.5330053756$ C, $0,0.5794100431,-0.8319611586,3.1885221353$ C, $0,-0.6709011365,-3.0228207047,3.4809081305$ C, $0,1.3286277889,-1.0557500912,4.5050722302$ $\mathrm{H}, 0,-0.3490162895,-0.2817971906,3.4000314318$ C, $0,0.0754526874,-3.2438064808,4.8087792353$ H,0,-1.6209633937,-2.5080535198,3.6746949669 C, $0,0.4827541011,-1.9151131174,5.4594018358$ $\mathrm{H}, 0,1.5561185773,-0.0936874805,4.9794711134$ $\mathrm{H}, 0,2.2879754136,-1.5542977041,4.3025142287$ $\mathrm{H}, 0,-0.5660205763,-3.8148772584,5.4899061997$ H,0,0.9697209704,-3.8584120114,4.6278245596 $\mathrm{H}, 0,1.0493013897,-2.1010273641,6.3798891721$ $\mathrm{H}, 0,-0.4232095863,-1.3619889359,5.7363348808$ $\mathrm{H}, 0,1.1185512268,-2.7130108528,2.318064351$ $\mathrm{H}, 0,-0.8936787463,-3.9985473872,3.0337376197$ C, $0,-1.5056080821,-0.5625323249,-3.9790284912$ C, $0,-0.008605736,-0.8488357468,-4.2448074787$ $\mathrm{H}, 0,0.1324846392,-1.8833831556,-4.581710345$ $\mathrm{H}, 0,0.3663217673,-0.1850833257,-5.0341316451$ $\mathrm{H}, 0,0.5910519571,-0.689501307,-3.3483425036$ $\mathrm{C}, 0,-2.2554359455,-0.7611788806,-5.3123612233$ $\mathrm{H}, 0,-1.8445923665,-0.0718211305,-6.0587801151$ $\mathrm{H}, 0,-2.1389622185,-1.7775808109,-5.7062799155$ $\mathrm{H}, 0,-3.3269329819,-0.5468823757,-5.2225357115$ C, $0,-1.6982730448,0.9144027212,-3.5601274158$ $\mathrm{H}, 0,-2.7636071163,1.1402548581,-3.4320279259$ $\mathrm{H}, 0,-1.1815956645,1.1340015357,-2.6243407416$ $\mathrm{H}, 0,-1.3051976937,1.579680984,-4.33927813$ 
C, $0,3.5744326157,1.476824676,-2.5375304172$

C,0,3.5819815612,-0.0149771187,-2.9507187033

$\mathrm{H}, 0,2.8145214953,-0.5793677129,-2.4191249135$

$\mathrm{H}, 0,3.3973266322,-0.1084509595,-4.0281800341$

$\mathrm{H}, 0,4.5581900408,-0.4676717318,-2.7369047362$

C, $0,2.2222957665,2.1311256122,-2.9107303409$

$\mathrm{H}, 0,1.3906841529,1.6432748965,-2.4012832167$

$\mathrm{H}, 0,2.2226024691,3.1951387988,-2.6427262736$

H, $0,2.0566532582,2.0581670925,-3.9929578232$

C, $0,4.6690139049,2.185090303,-3.3626658262$

$\mathrm{H}, 0,4.443255046,2.0676371253,-4.428632181$

$\mathrm{H}, 0,4.7162428637,3.2603376221,-3.153540384$

$\mathrm{H}, 0,5.6631721153,1.7566892714,-3.1892527846$

C,0,-4.6998520553,-4.2896988607,-2.5881095338

C, $0,-4.2363642621,-5.1814459467,-3.7649607765$

$\mathrm{H}, 0,-5.0436867122,-5.8573866574,-4.0725242019$

$\mathrm{H}, 0,-3.9496456137,-4.58740755,-4.6396652277$

H,0,-3.373128987,-5.792350936,-3.4761159611

C, $0,-5.1312452778,-5.2074736684,-1.4283218764$

$\mathrm{H}, 0,-4.3104219649,-5.8505607237,-1.0902634537$

$\mathrm{H}, 0,-5.492772247,-4.6319022385,-0.5683548817$

H, $0,-5.9473846462,-5.8599508394,-1.7585756587$

C, $0,-5.9303917375,-3.4608067962,-3.0280851016$

$\mathrm{H}, 0,-6.286993497,-2.8290024221,-2.2063990878$

$\mathrm{H}, 0,-5.7025061381,-2.809343902,-3.8787678492$

$\mathrm{H}, 0,-6.7497486806,-4.1253197117,-3.3286228109$

C, $0,6.5125215724,3.1765725659,1.2739189121$

C, $0,7.7637925363,2.4859033224,0.6802678964$

$\mathrm{H}, 0,7.7287714101,2.4475783535,-0.41388949$

$\mathrm{H}, 0,8.6718046888,3.0314037737,0.9652928473$

$\mathrm{H}, 0,7.8549578032,1.4577767082,1.049725315$

C,0,6.674124816,3.2296889381,2.8049276914

$\mathrm{H}, 0,5.8251739892,3.7287556675,3.2864210541$

$\mathrm{H}, 0,6.7741920172,2.2282763458,3.239466027$

$\mathrm{H}, 0,7.5785918886,3.7929000128,3.0609825158$

C, $0,6.4338998998,4.6298642417,0.7478537844$

$\mathrm{H}, 0,6.3557411009,4.6661925721,-0.3441936549$

$\mathrm{H}, 0,5.5636020886,5.1500582899,1.1646824778$

$\mathrm{H}, 0,7.3335691533,5.1879203567,1.0351141048$

C, $0,-5.2395248795,2.715777709,-0.6061194452$

C, $0,-6.5850920243,3.1028412747,-0.7530547473$

C, $0,-4.3188437087,3.0251822418,-1.6168554964$

C, $0,-6.9916860842,3.7970423426,-1.891993387$

H,0,-7.3180847136,2.8570692201,0.0093126192

C, $0,-4.7319873845,3.7233216789,-2.7536372815$

$\mathrm{H}, 0,-3.2855540348,2.7105059239,-1.52087108$

C,0,-6.0655867328,4.1105267659,-2.8914494802 
$\mathrm{H}, 0,-8.0317752954,4.0899813172,-1.9989500448$

$\mathrm{H}, 0,-4.0105550661,3.9579659562,-3.5304553559$

$\mathrm{H}, 0,-6.3863467047,4.6510255137,-3.7772236493$

C, $0,-4.8461502845,2.9476185589,2.3175097913$

C, $0,-4.228106301,2.5839063814,3.5266392543$

C, $0,-5.5756042997,4.1455278564,2.2434133767$

C,0,-4.3595510073,3.4040500915,4.6473711261

$\mathrm{H}, 0,-3.6496367778,1.6680815291,3.6181001174$

C, $0,-5.69273565,4.9628784578,3.3685568752$

$\mathrm{H}, 0,-6.0403594697,4.4554566867,1.3150454366$

C, $0,-5.0887089617,4.5927765286,4.5719944178$

$\mathrm{H}, 0,-3.8866598394,3.1043637074,5.5777673667$

H,0,-6.2542247948,5.8900201862,3.2996537515

$\mathrm{H}, 0,-5.1826198989,5.2310886015,5.4460178593$

C, $0,-5.7400165099,0.3614423133,1.1068144297$

C, $0,-6.5855773382,-0.0839936782,0.0785591854$

C, $0,-5.670442435,-0.357365782,2.3130014788$

C,0,-7.3704359156,-1.2239129839,0.2643293202

$\mathrm{H}, 0,-6.6367777493,0.4479857804,-0.8644198648$

C,0,-6.4549350506,-1.4979580437,2.485036774

$\mathrm{H}, 0,-5.0035581982,-0.0613505896,3.1188912218$

C, $0,-7.3089200307,-1.9299787835,1.4672800152$

$\mathrm{H}, 0,-8.0293004128,-1.5554626479,-0.5328537518$

H,0,-6.3917622941,-2.0471167999,3.419728696

$\mathrm{H}, 0,-7.9224854958,-2.8150334037,1.6101472253$

$\mathrm{Cl}, 0,-3.1723846517,-0.4924306946,5.1198010013$

$\mathrm{HF}=-3353.7820902$ (A.U.)

Zero-point correction $=1.163254$ (A.U.)

Total Gibbs free energy $=-3352.721919$ (A.U.)

$[\mathbf{2} \mathbf{a} /(R, R)-\mathbf{1}-\mathrm{Cl}]^{+}$

Cartesian Coordinates (Angstroms)

C, $0,-4.5434286929,-0.4786504601,-2.1128875698$

C, $0,-3.1700880496,-0.6795069269,-2.0003878772$

C, $0,-2.4528342343,0.1635323912,-1.0944411941$

C, $0,-3.1684106121,1.2007892667,-0.4275096643$

C, $0,-4.5656169655,1.3547569488,-0.6045083522$

C, $0,-5.2858659329,0.5141823839,-1.4286193742$

$\mathrm{H}, 0,-5.0910292916,-1.1271626658,-2.7849774476$

$\mathrm{H}, 0,-5.0543671188,2.1647116872,-0.0715455533$

C,0,-2.4994029074,2.1901197262,0.3651022637

$\mathrm{N}, 0,-1.2339680227,2.1964560988,0.6535952211$

$\mathrm{N}, 0,1.3096559349,2.3433002816,0.1894534516$

C, $0,2.5986674424,2.2786441534,-0.0156994289$

C,0,3.3593098672,1.1233833182,-0.3629164271 
C, $0,2.7459156409,-0.14040017,-0.6242256066$

C, $0,4.7640298304,1.2949252211,-0.4778495411$

C, $0,3.5875160131,-1.2292684915,-1.0290243692$

C, $0,5.5896639801,0.2537217387,-0.8408037405$

$\mathrm{H}, 0,5.1692147725,2.2800567432,-0.2683347963$

C,0,4.954246422,-0.985935204,-1.110006503

$\mathrm{H}, 0,5.5929343436,-1.8075157753,-1.4093579695$

$\mathrm{O}, 0,1.442204411,-0.2895405105,-0.5152625535$

$\mathrm{O}, 0,-1.1509951114,-0.0082138498,-0.9030905563$

Al,0,0.0964186514,0.7429247838,0.1877103816

$\mathrm{H}, 0,-3.1257670189,3.0120438847,0.7180041787$

$\mathrm{H}, 0,3.1789135633,3.1978037834,0.0748657441$

$\mathrm{P}, 0,-1.1164753729,-3.3706492703,3.390526237$

C, $0,0.4122275099,-0.5369880764,4.1545877339$

$\mathrm{H}, 0,-0.1250880181,0.3318596196,4.5515050458$

$\mathrm{H}, 0,1.4719835725,-0.2640716966,4.1059664964$

$\mathrm{H}, 0,0.2914368745,-1.3654513957,4.8537865796$

$\mathrm{O}, 0,0.1362194226,0.1579859658,1.933998952$

C, $0,-0.080586119,-0.8253834747,2.7538461093$

C, $0,-0.7131869079,-1.9913174038,2.3720495821$

$\mathrm{H}, 0,-1.0262569162,-2.0954777444,1.3370880496$

C, $0,0.6631240304,3.6424922721,0.4935049293$

C, $0,-0.5840589338,3.3142690316,1.3491113312$

C, $0,1.5287767282,4.7102108107,1.1772233342$

C,0,-1.4196329304,4.566732351,1.6249545872

$\mathrm{H}, 0,-0.2217656748,2.9143217598,2.3077550844$

C, $0,0.6951937978,5.9659576027,1.4876087467$

$\mathrm{H}, 0,1.9489027596,4.2948719176,2.1042243129$

C, $0,-0.5566381626,5.6386045179,2.3121850927$

$\mathrm{H}, 0,-2.2797716061,4.3198986016,2.2592851804$

$\mathrm{H}, 0,-1.817754356,4.9637377816,0.6803192924$

$\mathrm{H}, 0,1.3190230737,6.6941552669,2.0178864272$

$\mathrm{H}, 0,0.3953953674,6.442300551,0.5436877026$

$\mathrm{H}, 0,-1.15220249,6.5443009699,2.4722646355$

$\mathrm{H}, 0,-0.2574827484,5.2810703441,3.3075549686$

$\mathrm{H}, 0,0.2958979087,4.0478725003,-0.4623226695$

$\mathrm{H}, 0,2.3733425142,4.9960572021,0.5404769507$

C, $0,3.0034865388,-2.6125177461,-1.3834421727$

C, $0,2.0449091323,-2.4699078563,-2.5893344729$

$\mathrm{H}, 0,2.5855639138,-2.1025079196,-3.4696066816$

H,0,1.6141942905,-3.4460098297,-2.8453191214

$\mathrm{H}, 0,1.2289935389,-1.7800199759,-2.3728526249$

C,0,4.0943979989,-3.6285888382,-1.7799930143

$\mathrm{H}, 0,3.6194058218,-4.5855921505,-2.0225818059$

$\mathrm{H}, 0,4.6541664823,-3.3104354968,-2.6666692828$

$\mathrm{H}, 0,4.8079920746,-3.8138769422,-0.9682869544$

C, $0,2.2498423722,-3.2028402575,-0.1676193367$ 
H,0,2.9350330227,-3.3516856749,0.6763098629

$\mathrm{H}, 0,1.4379046939,-2.55065393,0.1567845811$

$\mathrm{H}, 0,1.8296362443,-4.1826810103,-0.4301369378$

C, $0,-2.4493990513,-1.7505850142,-2.8455851735$

C, $0,-1.4061661534,-1.0626221846,-3.7600254622$

$\mathrm{H}, 0,-0.6691398453,-0.5060377242,-3.1790267439$

$\mathrm{H}, 0,-0.8770944335,-1.8138578555,-4.3586512417$

$\mathrm{H}, 0,-1.8988296728,-0.3693246047,-4.452028422$

C, $0,-1.7582534475,-2.7847929073,-1.9258426714$

$\mathrm{H}, 0,-1.0050600666,-2.3136681457,-1.2924676761$

$\mathrm{H}, 0,-2.4954374351,-3.2855660045,-1.2866024548$

$\mathrm{H}, 0,-1.2634071905,-3.5525178891,-2.5335103376$

C, $0,-3.4203547998,-2.5275278797,-3.758834634$

$\mathrm{H}, 0,-2.8530295801,-3.2637410655,-4.3387728018$

$\mathrm{H}, 0,-4.1803818676,-3.0748151913,-3.1884087833$

$\mathrm{H}, 0,-3.9304179778,-1.8733603357,-4.4750234099$

C,0,7.1167071448,0.3786217966,-0.9751989875

C, $0,7.5356587458,0.0617628826,-2.4309538065$

$\mathrm{H}, 0,8.6238987613,0.1387330719,-2.5402217192$

H,0,7.2445746594,-0.9508493728,-2.7302588056

$\mathrm{H}, 0,7.0735076614,0.7641384758,-3.1339037018$

C,0,7.6114545176,1.7958023452,-0.6296404667

$\mathrm{H}, 0,7.1852686686,2.5518876898,-1.2992867163$

$\mathrm{H}, 0,7.3676140158,2.0724375108,0.4029622375$

$\mathrm{H}, 0,8.7007460568,1.8428131914,-0.7339993591$

C,0,7.805967421,-0.6193604493,-0.0139432729

$\mathrm{H}, 0,7.5370521947,-0.4104766032,1.0280572204$

$\mathrm{H}, 0,7.5290170865,-1.6568240843,-0.230614558$

$\mathrm{H}, 0,8.8959496037,-0.543230497,-0.1048288191$

C, $0,-6.8046327966,0.6238063254,-1.6450754976$

C, $0,-7.1002610623,0.8866050745,-3.1411849401$

$\mathrm{H}, 0,-6.7269391111,0.080199302,-3.7813010102$

$\mathrm{H}, 0,-8.1810998903,0.9667291306,-3.3074694849$

$\mathrm{H}, 0,-6.6353120046,1.8212233241,-3.4748390431$

C, $0,-7.4239411073,1.772219916,-0.8256876687$

$\mathrm{H}, 0,-7.2717621677,1.6349654975,0.2515399197$

$\mathrm{H}, 0,-7.0106400026,2.7467836796,-1.110751121$

$\mathrm{H}, 0,-8.5042438781,1.8109763369,-1.0023859049$

C,0,-7.4834808052,-0.6980247527,-1.2129406685

$\mathrm{H}, 0,-7.1100665273,-1.555265143,-1.7836544057$

$\mathrm{H}, 0,-7.3072520838,-0.900465039,-0.1499648545$

$\mathrm{H}, 0,-8.5666812362,-0.6403120169,-1.3728112416$

C, $0,-2.2432759123,-4.4401573656,2.4400084775$

C, $0,-3.546691151,-4.7109319295,2.8801761776$

C, $0,-1.7728682838,-5.0189236091,1.2483867599$

C, $0,-4.373899344,-5.5490320139,2.1297302336$

$\mathrm{H}, 0,-3.9177249005,-4.27446463,3.801236696$ 
C, $0,-2.6065623203,-5.8519246635,0.5046049837$ $\mathrm{H}, 0,-0.7613467298,-4.8256723814,0.9024017251$ C,0,-3.9065489657,-6.1176964721,0.9445164367 $\mathrm{H}, 0,-5.3823979493,-5.7563868738,2.4749230648$ $\mathrm{H}, 0,-2.2397765385,-6.2954300364,-0.4160632161$ $\mathrm{H}, 0,-4.5521033274,-6.7703545046,0.364068808$ C, $0,-1.9646807697,-2.8576913052,4.9239367514$ C, $0,-1.6991654684,-3.4605393575,6.1626287686$ C, $0,-2.9306606378,-1.8390674109,4.8387602684$ C, $0,-2.3970474575,-3.0518666068,7.3006436313$ $\mathrm{H}, 0,-0.9461926353,-4.2367495915,6.2465727867$ C, $0,-3.627672911,-1.4417555718,5.978538508$ $\mathrm{H}, 0,-3.1280580648,-1.3557470916,3.8863068464$ C, $0,-3.3615007271,-2.0471616231,7.2099306584$ $\mathrm{H}, 0,-2.1827373328,-3.5183426968,8.2575370588$ $\mathrm{H}, 0,-4.3745410541,-0.6565698913,5.9066837311$ $\mathrm{H}, 0,-3.9022804823,-1.7321203689,8.0975865248$ C, $0,0.3156506682,-4.4127512319,3.8334334195$ C, $0,0.1113286467,-5.7205750496,4.3090046409$ C, $0,1.6209243632,-3.9233783196,3.6863116452$ C, $0,1.2034998372,-6.5144811044,4.6553102309$ $\mathrm{H}, 0,-0.8939505059,-6.1235097232,4.3953482108$ C,0,2.7099384105,-4.7248001326,4.0334725254 $\mathrm{H}, 0,1.7864070263,-2.9309232809,3.2813104546$ C,0,2.5025919918,-6.0158295758,4.5213328938 $\mathrm{H}, 0,1.0411474594,-7.5242817984,5.0202805407$ $\mathrm{H}, 0,3.719000906,-4.3416490263,3.9142134243$ $\mathrm{H}, 0,3.3516657337,-6.6387119959,4.7871748743$

$\mathrm{HF}=-2893.3732026$ (A.U.)

Zero-point correction $=1.163290$ (A.U.)

Total Gibbs free energy $=-2892.312022$ (A.U.)

$[\mathbf{2} \mathrm{a} /(R, R)-\mathbf{1}-\mathrm{Cl}]^{+}$(optimized in the solvent ) Cartesian Coordinates (Angstroms)

C, $0,3.0332612279,-2.8315817799,-2.4826660199$

C, $0,1.7799047657,-2.2250632737,-2.4293621204$

C, $0,1.1240332013,-2.1753668592,-1.1584601109$

C, $0,1.7637241693,-2.7897730363,-0.0430027839$

C, $0,3.0353594492,-3.396450758,-0.1743153093$

C,0,3.7031975983,-3.4204186568,-1.3838676646

$\mathrm{H}, 0,3.5361721503,-2.8620512984,-3.4411782673$

$\mathrm{H}, 0,3.4686038758,-3.8495532263,0.7117815812$

C, $0,1.1186436517,-2.900223168,1.2370789616$

$\mathrm{N}, 0,-0.0301837278,-2.3848229558,1.5434973067$

N,0,-2.6065123092,-2.1420115541,1.4001535639 
C, $0,-3.8833932009,-1.9276026937,1.2392086752$

C, $0,-4.4932160849,-1.0386886044,0.2984711446$

C, $0,-3.7317126203,-0.3135521899,-0.6670552287$

C, $0,-5.9040263091,-0.9081789569,0.3647946775$

C, $0,-4.4268054915,0.5824228282,-1.5459483143$

C, $0,-6.590905672,-0.0639913609,-0.4829944526$

$\mathrm{H}, 0,-6.4266515609,-1.4955518946,1.1129565742$

C, $0,-5.8092182643,0.6658260917,-1.4147674284$

$\mathrm{H}, 0,-6.3378057495,1.3411227571,-2.0763487793$

$\mathrm{O}, 0,-2.4295441493,-0.4782215176,-0.7582785715$

$\mathrm{O}, 0,-0.0576302237,-1.5838434419,-1.035456596$

Al, $0,-1.1660140233,-1.2040245203,0.3614811708$

$\mathrm{H}, 0,1.648488674,-3.4945690821,1.9830330997$

$\mathrm{H}, 0,-4.5813910326,-2.4647551309,1.881859476$

$\mathrm{P}, 0,2.4274396054,2.6542717441,0.8698314994$

C, $0,-0.3548046026,2.2106713203,2.6339658625$

$\mathrm{H}, 0,-0.3580476881,1.6502033974,3.575937442$

$\mathrm{H}, 0,-1.3790656831,2.5572635356,2.4601334281$

$\mathrm{H}, 0,0.2961061037,3.0777107749,2.7496374914$

$\mathrm{O}, 0,-0.8150238912,0.3166046188,1.3301237588$

C, $0,0.0395696133,1.2847214447,1.5072556059$

C, $0,1.1742150129,1.4207546608,0.7363187919$

$\mathrm{H}, 0,1.3475462534,0.705972723,-0.0629169383$

C, $0,-2.1336843953,-3.1097722612,2.4227668368$

C, $0,-0.7076049829,-2.6571885104,2.8188504197$

C, $0,-3.0131388824,-3.2837569284,3.6684543559$

C, $0,-0.0502953258,-3.6500868363,3.7794614286$

$\mathrm{H}, 0,-0.8038743056,-1.6880081802,3.3294217062$

C, $0,-2.3520054935,-4.2533440826,4.6642109613$

$\mathrm{H}, 0,-3.1696116421,-2.3036580233,4.1396323091$

C, $0,-0.9256818353,-3.8227109245,5.0318790039$

$\mathrm{H}, 0,0.9471637814,-3.2965670647,4.0670521325$

H,0,0.0788859443,-4.6214424703,3.2821699361

$\mathrm{H}, 0,-2.9715584991,-4.3239580236,5.5654019921$

$\mathrm{H}, 0,-2.3248850969,-5.2589853728,4.2221435286$

$\mathrm{H}, 0,-0.4682049001,-4.5604768236,5.7007212311$

$\mathrm{H}, 0,-0.9590611408,-2.8734962227,5.5845063599$

$\mathrm{H}, 0,-2.0346418473,-4.0833950303,1.9198847025$

$\mathrm{H}, 0,-3.9994899371,-3.6751310266,3.3980466354$

C, $0,-3.6703313392,1.4235590267,-2.5951331637$

C, $0,-2.9690593411,0.4870730186,-3.607096083$

$\mathrm{H}, 0,-3.7081574922,-0.1188158996,-4.1458035868$

$\mathrm{H}, 0,-2.4177697745,1.0800955689,-4.3478959536$

$\mathrm{H}, 0,-2.2666732615,-0.1825951339,-3.1102373855$

C,0,-4.6111721132,2.3463410447,-3.3970516103

$\mathrm{H}, 0,-4.017477678,2.9206405466,-4.1171163766$

$\mathrm{H}, 0,-5.3592798286,1.7821025143,-3.9657795608$ 
$\mathrm{H}, 0,-5.1347169577,3.0645451146,-2.7550616281$ C, $0,-2.6277756047,2.3312046455,-1.8957102885$ $\mathrm{H}, 0,-3.1249577917,3.030018364,-1.2112766905$ $\mathrm{H}, 0,-1.9031278664,1.7466796347,-1.3268912461$ $\mathrm{H}, 0,-2.0862973043,2.9238498467,-2.6437987187$ C, $0,1.1226728193,-1.6419878139,-3.6983911956$ C, $0,-0.2215693739,-2.3619262874,-3.9665641276$ $\mathrm{H}, 0,-0.911677961,-2.2432351726,-3.1299984825$ $\mathrm{H}, 0,-0.6937362911,-1.9492893032,-4.8666299247$ $\mathrm{H}, 0,-0.0573532375,-3.4333602613,-4.1352101079$ C, $0,0.8907336027,-0.1216277317,-3.5267817337$ $\mathrm{H}, 0,0.2428540509,0.0915767656,-2.6755296074$ $\mathrm{H}, 0,1.8461618577,0.395994959,-3.377845321$ $\mathrm{H}, 0,0.4219750309,0.2903405409,-4.429004889$ C,0,2.0012023252,-1.8316718851,-4.9526919523 $\mathrm{H}, 0,1.4807134346,-1.4091506886,-5.8193883559$ $\mathrm{H}, 0,2.9654284255,-1.3170334211,-4.8665592216$ H,0,2.192827153,-2.8892095065,-5.1685723076 C, $0,-8.1189222141,0.1128830738,-0.4591383169$ C,0,-8.7066082499,-0.2821896666,-1.8352040059 $\mathrm{H}, 0,-9.7957549454,-0.1528112949,-1.8356372792$ $\mathrm{H}, 0,-8.2990969671,0.3321605276,-2.6453191062$ $\mathrm{H}, 0,-8.4885738477,-1.3312911353,-2.0667129707$ C, $0,-8.7852179892,-0.7652162594,0.6169352451$ $\mathrm{H}, 0,-8.5954222198,-1.8315065088,0.4475039025$ $\mathrm{H}, 0,-8.4364717706,-0.5102906303,1.6244851405$ $\mathrm{H}, 0,-9.8703792527,-0.6151711098,0.5947350026$ C, $0,-8.4680157632,1.5905902136,-0.1595738381$ $\mathrm{H}, 0,-8.0751898628,1.894728278,0.8176807735$ $\mathrm{H}, 0,-8.0562165384,2.2697578016,-0.9140351987$ H, $0,-9.5559589605,1.7296721263,-0.1471173731$ C, $0,5.0888026887,-4.0596893569,-1.5802627711$ C, $0,4.982485375,-5.2256397325,-2.5924741074$ H,0,4.6118028926,-4.8881965549,-3.5664056232 H,0,5.966466145,-5.6837206075,-2.7511992194 $\mathrm{H}, 0,4.3016968607,-6.0020372443,-2.2243411827$ C, $0,5.6591397375,-4.6177788152,-0.2625789669$ $\mathrm{H}, 0,5.780957236,-3.8326550636,0.4928392124$ $\mathrm{H}, 0,5.0200853915,-5.4026927829,0.1581543518$ $\mathrm{H}, 0,6.645346444,-5.0586574416,-0.4458112721$ C,0,6.0779021006,-3.0007505476,-2.1236980712 H, 0,5.757237781,-2.6007148426,-3.0915990783 $\mathrm{H}, 0,6.172664083,-2.158624412,-1.4283752207$ $\mathrm{H}, 0,7.0715019204,-3.4444007647,-2.2610561123$ C, $0,3.8420154456,2.0909679026,-0.1312537591$ C, $0,5.0337529497,1.6525776892,0.4629494412$ C, $0,3.7157812043,2.0866337465,-1.531408051$ 
C, $0,6.0878569082,1.2082344964,-0.3383270042$ $\mathrm{H}, 0,5.1465012272,1.6590947443,1.5416466927$ C,0,4.771377761,1.6378779617,-2.323586285 $\mathrm{H}, 0,2.8036894198,2.438672413,-2.0040459238$ C,0,5.95787219,1.1990903146,-1.7281536961 $\mathrm{H}, 0,7.0106622666,0.8750713923,0.126895867$ $\mathrm{H}, 0,4.6695556515,1.638187021,-3.4045924128$ H,0,6.7810905748, $0.8565672121,-2.3481266486$ C, $0,2.979769761,2.8753280371,2.594567181$ C, $0,3.2858239693,4.1407607959,3.1178628119$ C,0,3.1209821071,1.734244465,3.4046702034 C, $0,3.7333695366,4.2605758094,4.4347232866$ $\mathrm{H}, 0,3.1683255266,5.0316041478,2.5107563583$ C, $0,3.5746338132,1.8623299084,4.7168018103$ $\mathrm{H}, 0,2.8694651835,0.7526636731,3.0147875853$ C, $0,3.8806147847,3.1247214418,5.2329529219$ $\mathrm{H}, 0,3.9627633579,5.2435197661,4.8348239702$ $\mathrm{H}, 0,3.6829232267,0.9774467818,5.3367353327$ $\mathrm{H}, 0,4.2281415121,3.2221326872,6.2572327719$ C, $0,1.9330285057,4.2739968946,0.1895251223$ C,0,2.910511481,5.234118236,-0.1289579621 C, $0,0.5782012834,4.5615908612,-0.029107073$ C, $0,2.5272309702,6.4743295592,-0.6378223222$ $\mathrm{H}, 0,3.9654875996,5.0140634237,0.005503634$ C, $0,0.2024648627,5.8050984244,-0.5404025193$ $\mathrm{H}, 0,-0.1791643526,3.8136650194,0.1798999558$ C, $0,1.1740631497,6.7616162421,-0.8405402302$ $\mathrm{H}, 0,3.28526739,7.2120547331,-0.8828604364$ $\mathrm{H}, 0,-0.8481128506,6.0205119905,-0.7099285905$ $\mathrm{H}, 0,0.8796830435,7.7271521285,-1.2412788878$

$\mathrm{HF}=-2893.4173217$ (A.U.)

Zero-point correction $=1.162218$ (A.U.)

Total Gibbs free energy $=-2892.357855$ (A.U.)

6a

Cartesian Coordinates (Angstroms)

C, $0,9.6555195045,-3.2710805994,-1.2709010212$ C, $0,8.6739469721,-4.012147052,-1.9373171392$ C, $0,7.3260964316,-3.7328210523,-1.7403315446$ C, $0,6.922092453,-2.701275588,-0.8685429086$ C,0,7.9243024363,-1.9661936524,-0.2066090632 C, $0,9.2744907169,-2.2460181855,-0.4039560815$ $\mathrm{H}, 0,10.7074941654,-3.4928210317,-1.4278487834$ $\mathrm{H}, 0,8.9634746932,-4.8118109226,-2.6138767764$ $\mathrm{H}, 0,6.5801804617,-4.3201624972,-2.2675011551$ 
C,0,5.5229361925,-2.3610631936,-0.6209614158

$\mathrm{H}, 0,7.631859769,-1.1664580913,0.4699198451$

H,0,10.028534799,-1.6639977284,0.118903126

C,0,4.4198506356,-2.9405731984,-1.1526927226

C, $0,3.0837756392,-2.5123799343,-0.8277100512$

C, $0,1.948670245,-3.0528715383,-1.3271194803$

C, $0,0.6149659983,-2.5433001555,-0.9323542243$

$\mathrm{O}, 0,0.4774955369,-1.6172999394,-0.1422580646$

C, $0,-0.5860747578,-3.2314743709,-1.5609737394$

$\mathrm{H}, 0,5.366737191,-1.539461192,0.0786041431$

$\mathrm{H}, 0,4.515454214,-3.7655317574,-1.8570681467$

$\mathrm{H}, 0,2.9710768857,-1.6877181081,-0.1240801822$

$\mathrm{H}, 0,1.9925051441,-3.8795664807,-2.0347103269$

$\mathrm{H}, 0,-1.5072085307,-2.7728821485,-1.1967438106$

$\mathrm{H}, 0,-0.5452349986,-3.1536543637,-2.6551386099$

H,0,-0.5877237979,-4.3017192184,-1.3170368665

$\mathrm{HF}=-539.7062128$ (A.U.)

Zero-point correction $=0.205356$ (A.U.)

Total Gibbs free energy $=-539.541323$ (A.U.)

\section{TS}

Cartesian Coordinates (Angstroms)

C, $0,-2.136082625,-1.147383991,-0.9379255964$

$\mathrm{O}, 0,-2.5643087237,0.0749688705,-0.8559631924$

Si, $0,-3.6884853889,0.6809835197,0.4271123479$

C,0,-3.0662036793,-1.5020196661,1.2593070995

$\mathrm{N}, 0,-2.8280215941,-2.5412455303,1.7526208997$

C, $0,-3.0142157696,-2.1971633993,-1.5523497582$

$\mathrm{H}, 0,-2.7266929172,-3.196768232,-1.2232711333$

$\mathrm{H}, 0,-4.0635487989,-2.022384137,-1.3188510345$

$\mathrm{H}, 0,-2.8849380614,-2.1297612642,-2.6439293506$

C, $0,-2.8110254614,1.258923075,2.0041238136$

$\mathrm{H}, 0,-2.9249932881,2.3420968756,2.1354772682$

$\mathrm{H}, 0,-3.2084830467,0.7385211186,2.8803786545$

$\mathrm{H}, 0,-1.7400920715,1.0298514095,1.9620030676$

C, $0,-5.4573137425,0.0070418594,0.5264732227$

$\mathrm{H}, 0,-5.6123553424,-0.5937991949,1.4259353352$

$\mathrm{H}, 0,-6.1713353509,0.8403307141,0.5093729925$

$\mathrm{H}, 0,-5.6892381344,-0.6314837888,-0.3347962934$

C, $0,-3.9405853041,2.3130451668,-0.5516795243$

$\mathrm{H}, 0,-4.5812658002,3.0001784115,0.0178136334$

$\mathrm{H}, 0,-2.9958149472,2.8365757131,-0.7469824949$

$\mathrm{H}, 0,-4.4269727318,2.1454875081,-1.5213264488$

C, $0,-0.7389481438,-1.4213674239,-0.7681174938$

$\mathrm{H}, 0,-0.4220053961,-2.4557076439,-0.862007874$ 
C, $0,0.1707492257,-0.4368599005,-0.5052040632$

$\mathrm{H}, 0,-0.1965066988,0.5846252705,-0.4247417922$

C, $0,1.5732379,-0.6529312206,-0.3401408988$

$\mathrm{H}, 0,1.93387423,-1.6768803523,-0.4100555395$

C, $0,2.4339920432,0.3723750437,-0.0948408202$

$\mathrm{H}, 0,2.0077520983,1.3741049754,-0.0362736196$

C, $0,3.8732031047,0.3031121187,0.1050104186$

$\mathrm{C}, 0,4.5790541959,1.5004466214,0.3394507715$

C, $0,4.6002840009,-0.9058752689,0.0767644295$

$\mathrm{C}, 0,5.9568161432,1.4947535922,0.5369602937$

$\mathrm{H}, 0,4.0324454643,2.4399782803,0.3658349063$

C,0,5.9755823884,-0.9099473241,0.2742963262

$\mathrm{H}, 0,4.0854609812,-1.8455513472,-0.0978545608$

$\mathrm{C}, 0,6.6597884793,0.2889756898,0.5047869081$

$\mathrm{H}, 0,6.4816082652,2.4286584463,0.7165747458$

$\mathrm{H}, 0,6.5198244496,-1.8497808872,0.2505779025$

$\mathrm{H}, 0,7.7350343781,0.2802243222,0.6594127521$

Only imaginary frequency: $-254.4 \mathrm{~cm}^{-1}$

$\mathrm{HF}=-1041.7703777$ (A.U.)

Zero-point correction $=0.326326$ (A.U.)

Total Gibbs free energy $=-1041.498185$ (A.U.)

$(S)-7 \mathbf{a}$

Cartesian Coordinates (Angstroms)

C, $0,-2.0867645033,-1.2159340254,-0.3929830562$

$\mathrm{O}, 0,-2.379474513,0.1601400976,-0.4953380301$

$\mathrm{Si}, 0,-3.6740132541,1.1059903583,0.060707257$

C, $0,-2.7369733243,-1.8248194938,0.8069342921$

$\mathrm{N}, 0,-3.2454433641,-2.3060623752,1.7338578291$

C, $0,-2.6009963166,-1.9516732643,-1.6514689939$

$\mathrm{H}, 0,-2.3406338498,-3.0144339785,-1.6211483259$

$\mathrm{H}, 0,-3.687663336,-1.8581776502,-1.7321306042$

$\mathrm{H}, 0,-2.1324846915,-1.4930791696,-2.5265067965$

C, $0,-3.5075976492,1.4223950368,1.9094362687$

$\mathrm{H}, 0,-4.3012955523,2.0970152152,2.2552020633$

$\mathrm{H}, 0,-3.5796036691,0.4968194327,2.4898003938$

$\mathrm{H}, 0,-2.5460802836,1.8939793691,2.1439818308$

C,0,-5.329410363,0.2894311621,-0.3297341799

$\mathrm{H}, 0,-6.1526099584,0.9423939575,-0.0125405632$

$\mathrm{H}, 0,-5.4486138755,0.1101630271,-1.4047855657$

$\mathrm{H}, 0,-5.4519598881,-0.6654118445,0.1933001708$

$\mathrm{C}, 0,-3.4727541002,2.7049323649,-0.9091767658$

$\mathrm{H}, 0,-4.245840899,3.431848706,-0.6312957106$

$\mathrm{H}, 0,-2.4977942881,3.1661756609,-0.7145782341$

$\mathrm{H}, 0,-3.549726673,2.5294844834,-1.9881363873$ 
C, $0,-0.5872930198,-1.4227624789,-0.2479143504$

$\mathrm{H}, 0,-0.2753972848,-2.4634564501,-0.1744475329$

C, $0,0.3185212126,-0.4318058162,-0.2313097948$

$\mathrm{H}, 0,-0.0336899197,0.5944173517,-0.3072711473$

C, $0,1.7484584414,-0.6413482305,-0.1269662506$

$\mathrm{H}, 0,2.0855939496,-1.6742157516,-0.0494849511$

C, $0,2.6444785573,0.3701356662,-0.1231809461$

$\mathrm{H}, 0,2.2550478144,1.3857334267,-0.2027035114$

C, $0,4.1019788413,0.2776324877,-0.0235394678$

C, $0,4.8582957985,1.4647747976,-0.0341451698$

C, $0,4.796848784,-0.9438245797,0.0824098541$

C, $0,6.2483359399,1.4385734952,0.0573291446$

H,0,4.3413482932,2.4183391124,-0.115409183

C,0,6.1845397458,-0.9706403092,0.17358632

$\mathrm{H}, 0,4.2471411589,-1.8804230844,0.093968387$

C,0,6.9190726874,0.2194614506,0.1615910332

$\mathrm{H}, 0,6.8072797461,2.3705630658,0.0470191043$

$\mathrm{H}, 0,6.698417335,-1.9249326078,0.2548564755$

$\mathrm{H}, 0,8.0029482712,0.1939733843,0.2330520943$

$\mathrm{HF}=-1041.848978$ (A.U.)

Zero-point correction $=0.329000 \quad$ (A.U.)

Total Gibbs free energy $=-1041.573480$ (A.U.)

$\mathrm{Me}_{3} \mathrm{P}(\mathrm{OTMS})(\mathrm{N}=\mathrm{C})$

Cartesian Coordinates (Angstroms)

Si, $0,1.5798117654,-1.1694384865,0.4354297953$

C, $0,-0.1744894105,2.1099804484,0.1733872257$

$\mathrm{N}, 0,-0.5837377665,1.0027627832,0.1121185318$

$\mathrm{P}, 0,-1.5400331657,-0.8325270706,-0.03118475$

$\mathrm{O}, 0,-0.0887275753,-1.4918035259,0.185986703$

C, $0,-2.4827156508,-0.2033728706,1.4201470262$

$\mathrm{H}, 0,-2.8821837958,0.7879755375,1.1983915092$

$\mathrm{H}, 0,-3.2840424883,-0.8937476614,1.6962648203$

$\mathrm{H}, 0,-1.7833671549,-0.0951646517,2.2532768764$

C, $0,-2.0171033415,-0.202609439,-1.6944903438$

$\mathrm{H}, 0,-2.703275716,-0.892389081,-2.1928347475$

$\mathrm{H}, 0,-2.4631312384,0.7890666937,-1.5988876154$

$\mathrm{H}, 0,-1.1047770349,-0.0949724064,-2.2869465591$

C, $0,-2.3674946924,-2.5043887141,-0.1554404368$

$\mathrm{H}, 0,-2.2126906638,-3.0796832761,0.7630090727$

$\mathrm{H}, 0,-3.4466066824,-2.3884305847,-0.3167962256$

$\mathrm{H}, 0,-1.9507083853,-3.0790813872,-0.9888110833$

C, $0,1.8478832957,-0.267660392,2.0618210226$

$\mathrm{H}, 0,2.9207543405,-0.2140060053,2.2872319135$

$\mathrm{H}, 0,1.4538779176,0.7510574486,2.0208524508$ 
$\mathrm{H}, 0,1.3657077231,-0.7960172758,2.8933709437$

C,0,2.2823652026,-2.9156301442,0.5405512199

H,0,2.096899218,-3.4776798755,-0.3820726418

$\mathrm{H}, 0,3.3672641946,-2.8904283103,0.7027778452$

$\mathrm{H}, 0,1.8351139835,-3.4775368694,1.3686592446$

C, $0,2.3117799895,-0.2677026085,-1.0414931922$

$\mathrm{H}, 0,1.9228419412,0.750940174,-1.1176676549$

H,0,3.4036443968,-0.2138240866,-0.9431678502

$\mathrm{H}, 0,2.0940757541,-0.7961979927,-1.9776688002$

$\mathrm{HF}=-1038.4468446$ (A.U.)

Zero-point correction $=0.240091$ (A.U.)

Total Gibbs free energy $=-1038.252069$ (A.U.)

$(R, R)-1$

Cartesian Coordinates (Angstroms)

C,0,3.4444277721,-2.2066182264,-1.3405861968

C, $0,2.1026161705,-2.1890381161,-0.9735454528$

C, $0,1.3399405455,-1.0232519644,-1.308361564$

C, $0,2.0087815869,0.0545726247,-1.9598942806$

C, $0,3.3820701727,-0.0307116111,-2.2923760132$

C, $0,4.1256110157,-1.1571910665,-2.0049871975$

H,0,4.0190081887,-3.0929356678,-1.1005154592

$\mathrm{H}, 0,3.8322910562,0.823719023,-2.7890358142$

C, $0,1.3393855572,1.2873883326,-2.2551638825$

$\mathrm{N}, 0,0.0782608765,1.5207852626,-2.0684843137$

$\mathrm{N}, 0,-2.142715542,1.8633745687,-0.7838581026$

C, $0,-3.2990759492,1.9075060582,-0.180191273$

C, $0,-4.1257243484,0.8007773726,0.1775278588$

C, $0,-3.6986488613,-0.5505230674,-0.0042544899$

C, $0,-5.3928233384,1.0997697243,0.7419199312$

C, $0,-4.5955745308,-1.6027395412,0.3851819312$

C,0,-6.2662562734,0.1029668862,1.1173568019

$\mathrm{H}, 0,-5.6547746659,2.1469179612,0.8611297751$

C, $0,-5.8242130783,-1.2313051775,0.9159175203$

H, $0,-6.5067775457,-2.0224582641,1.2012808946$

$\mathrm{O}, 0,-2.509744729,-0.8173895312,-0.4883262933$

$\mathrm{O}, 0,0.0586575596,-0.9489328035,-0.9961381252$

Al,0,-1.3118424665,0.1517483253,-1.4955777956

$\mathrm{H}, 0,1.9715169063,2.082288973,-2.6576640285$

$\mathrm{H}, 0,-3.7037803899,2.8848985967,0.0888978531$

C, $0,-1.3947760969,3.1102103095,-1.0630839755$

C, $0,-0.5377158164,2.8260595595,-2.3194692702$

C, $0,-2.2201488399,4.3919056361,-1.2458776596$

C, $0,0.3868944928,4.0010696302,-2.6477503563$

$\mathrm{H}, 0,-1.230794945,2.6780593518,-3.1607997746$ 
C,0,-1.3077310234,5.5785031702,-1.6042996107 $\mathrm{H}, 0,-2.9621044296,4.2310124537,-2.0403655379$ C, $0,-0.4373951011,5.2855232501,-2.8335189059$ $\mathrm{H}, 0,0.956887316,3.7927393294,-3.5611159986$ $\mathrm{H}, 0,1.1153581203,4.1415657825,-1.8358519378$ $\mathrm{H}, 0,-1.9207682524,6.4709271266,-1.7768310112$ $\mathrm{H}, 0,-0.6596783807,5.8070910912,-0.7459259604$ $\mathrm{H}, 0,0.2322691291,6.1302585131,-3.0334149413$ $\mathrm{H}, 0,-1.0794647778,5.1777993727,-3.718718181$ $\mathrm{H}, 0,-0.6942120797,3.2594209102,-0.2259153969$ $\mathrm{H}, 0,-2.7739277495,4.6355111286,-0.3321396793$ C,0,-4.2103395956,-3.0859790578,0.2127091029 C, $0,-2.9554344388,-3.3957668622,1.0632820658$ $\mathrm{H}, 0,-3.1579115802,-3.2267780119,2.1283912145$ $\mathrm{H}, 0,-2.6686408264,-4.4475585909,0.9383888469$ $\mathrm{H}, 0,-2.112007716,-2.7720596238,0.7674173754$ C, $0,-5.3302412932,-4.0393203177,0.6784924087$ $\mathrm{H}, 0,-5.0002400032,-5.0739442394,0.5322130873$ H, $0,-5.5645981033,-3.918133769,1.742966464$ H, $,-6.2540117809,-3.9071176208,0.1026520713$ C, $0,-3.9353310957,-3.3926031182,-1.2798596804$ $\mathrm{H}, 0,-4.8352807076,-3.2194558544,-1.8820844116$ $\mathrm{H}, 0,-3.1341929443,-2.7720320026,-1.6820276777$ $\mathrm{H}, 0,-3.6503135374,-4.4457199043,-1.3968186468$ C, $0,1.4593810495,-3.3802524298,-0.2347304274$ C, $0,0.935182106,-2.9115481337,1.1450776778$ $\mathrm{H}, 0,0.2009686219,-2.1113318936,1.0424563998$ $\mathrm{H}, 0,0.4611368541,-3.749520932,1.6709752858$ $\mathrm{H}, 0,1.7620746396,-2.5486086329,1.768334224$ C, $0,0.3016823245,-3.965074388,-1.0797754387$ $\mathrm{H}, 0,-0.4792380097,-3.2256886082,-1.2576727029$ $\mathrm{H}, 0,0.6724089129,-4.3187297857,-2.04948791$ H,0,-0.145561533,-4.8205493777,-0.5583292735 C, $0,2.4643843977,-4.522260804,0.0205082871$ $\mathrm{H}, 0,1.9543544221,-5.3336355415,0.5516178789$ $\mathrm{H}, 0,2.8621141364,-4.9415809944,-0.9114243161$ $\mathrm{H}, 0,3.3079889271,-4.2027250311,0.6440734$ C, $0,-7.6563035756,0.3654913482,1.7205003789$ C,0,-7.7495034694,-0.2865001306,3.1208284156 $\mathrm{H}, 0,-8.7396179625,-0.1111259349,3.5596904106$ $\mathrm{H}, 0,-7.5934119077,-1.3697005897,3.0790215994$ H,0,-6.9973938318,0.1329224164,3.7989944881 C,0,-7.944693079,1.8709126036,1.8742810108 $\mathrm{H}, 0,-7.2191642445,2.3581050877,2.5361900541$ $\mathrm{H}, 0,-7.9311308431,2.3890990811,0.9083351833$ H,0,-8.9391686768,2.0147748117,2.3115467337 
C, $0,-8.7432630482,-0.2405151027,0.800393221$

$\mathrm{H}, 0,-8.7090384767,0.2138728964,-0.196388838$

$\mathrm{H}, 0,-8.6180686224,-1.3215293453,0.6779640486$

$\mathrm{H}, 0,-9.7418626892,-0.0671309853,1.2205842266$

C, $0,5.6128191306,-1.3139671891,-2.364568786$

C,0,6.4398114002,-1.5577920699,-1.0794349343

$\mathrm{H}, 0,6.1187405609,-2.462036006,-0.5516886547$

$\mathrm{H}, 0,7.5022660165,-1.6773733614,-1.3254671585$

$\mathrm{H}, 0,6.343927685,-0.7147145985,-0.3854358285$

C,0,6.1737801328,-0.0610606986,-3.0636095324

H,0,5.6492308532,0.1497010853,-4.0027244063

$\mathrm{H}, 0,6.104768635,0.8271053931,-2.4244905568$

$\mathrm{H}, 0,7.2322017672,-0.212951703,-3.30363258$

C, $0,5.7880788562,-2.5178358638,-3.3211565819$

$\mathrm{H}, 0,5.4331573822,-3.4511140906,-2.8709960912$

$\mathrm{H}, 0,5.2288717787,-2.3626973649,-4.2508726553$

$\mathrm{H}, 0,6.8461951707,-2.653341136,-3.5776737136$

$\mathrm{Cl}, 0,-2.1067854238,-0.1822403148,-3.5646548452$

$\mathrm{HF}=-2125.5328859$ (A.U.)

Zero-point correction $=0.821689$ (A.U.)

Total Gibbs free energy $=-2124.786247$ (A.U.)

\section{model I}

Cartesian Coordinates (Angstroms)

C, $0,3.6613055263,1.8046188682,-0.8536275776$

C, $0,2.3668462788,1.3804011395,-1.1249136394$

C, $0,1.6694539242,0.6470598043,-0.1022977916$

C, $0,2.3569320088,0.400109173,1.1280809602$

C, $0,3.680276086,0.8656859575,1.3288177498$

C, $0,4.3607702658,1.5718522957,0.3571159809$

$\mathrm{H}, 0,4.1793819691,2.3631234909,-1.6239073471$

H,0,4.1409473361,0.6593812885,2.2909669946

C, $0,1.7434316314,-0.2854505179,2.233558917$

$\mathrm{N}, 0,0.5566328499,-0.7991328648,2.2498086654$

$\mathrm{N}, 0,-1.5401937403,-2.2961311294,1.7638277982$

C, $0,-2.6982399234,-2.8152107902,1.4991751024$

C, $0,-3.523601878,-2.5159559883,0.3616871125$

C, $0,-3.0856333445,-1.6679180352,-0.7035787052$

C, $0,-4.7997510564,-3.1297892755,0.3277014907$

C, $0,-3.9874040644,-1.4445541774,-1.7990783001$

C, $0,-5.6754499811,-2.9275982743,-0.7188591274$

H,0,-5.0714812764,-3.7654628196,1.1654921366

C, $0,-5.2254027169,-2.0751781457,-1.7576819468$

$\mathrm{H}, 0,-5.9068048543,-1.9003796479,-2.5816804401$

$\mathrm{O}, 0,-1.8843326607,-1.1351356208,-0.7035019857$ 
$\mathrm{O}, 0,0.4440912704,0.2338342843,-0.3075715434$

Al,0,-0.7481991749,-0.7452573788,0.7011165472

$\mathrm{H}, 0,2.3598983537,-0.3523691809,3.1333922571$

H,0,-3.1129937834,-3.5582194915,2.1843032482

C, $0,9.852841276,-3.7911512442,-1.2867233308$

C, $0,8.8936204335,-4.5613339254,-1.9540072098$

C, $0,7.5382642992,-4.3177570488,-1.761442207$

C,0,7.1056605738,-3.2937648972,-0.8933709477

C, $0,8.0856660032,-2.5282294787,-0.2310862143$

C,0,9.4433672467,-2.772847894,-0.4241093563

$\mathrm{H}, 0,10.9106857139,-3.9844726169,-1.4412401939$

H,0,9.2069848008,-5.3536863295,-2.6282501656

H,0,6.8092902998,-4.923173241,-2.2916005573

C, $0,5.6990474264,-2.9912851117,-0.6491734833$

$\mathrm{H}, 0,7.770403754,-1.7349099824,0.4423159859$

$\mathrm{H}, 0,10.180650026,-2.1687884714,0.0972540159$

C,0,4.6109163266,-3.6317008498,-1.1470764433

C, $0,3.2725490105,-3.229540024,-0.8143067159$

C,0,2.1411015542,-3.8188039142,-1.2801232724

C,0,0.811695172,-3.3370584129,-0.8916070497

$\mathrm{O}, 0,0.688267877,-2.4379854828,-0.0475326033$

C, $0,-0.389782993,-3.9551207762,-1.5687366411$

$\mathrm{H}, 0,5.5160337183,-2.1462001166,0.0149563884$

$\mathrm{H}, 0,4.7250036848,-4.4816822665,-1.8175350286$

$\mathrm{H}, 0,3.1544254116,-2.3829380429,-0.1389187104$

H,0,2.1963900522,-4.6541712984,-1.9744359814

$\mathrm{H}, 0,-1.2529201838,-3.9480888123,-0.9013563587$

$\mathrm{H}, 0,-0.6528049017,-3.3476555997,-2.4438523243$

$\mathrm{H}, 0,-0.1833394763,-4.9733112745,-1.9126319339$

C, $0,-0.7453386161,-2.7089194672,2.9326030607$

C, $0,-0.0197782653,-1.4371392905,3.4402833507$

C, $0,-1.4961458193,-3.4093815207,4.0727874828$

C, $0,0.9330349751,-1.7604399918,4.5955057668$

$\mathrm{H}, 0,-0.7954586786,-0.7456166452,3.7993507273$

C, $0,-0.5463078443,-3.7211962153,5.2411626702$

$\mathrm{H}, 0,-2.3153026822,-2.7625015001,4.416824401$

C, $0,0.1784518703,-2.462969283,5.736118314$

$\mathrm{H}, 0,1.3955366379,-0.841555078,4.9753899859$

$\mathrm{H}, 0,1.7472651658,-2.4069675192,4.2360344347$

$\mathrm{H}, 0,-1.1102031311,-4.1829106442,6.060513979$

$\mathrm{H}, 0,0.1964026115,-4.4639301165,4.9156596099$

$\mathrm{H}, 0,0.8786920344,-2.7193353456,6.5402319576$

$\mathrm{H}, 0,-0.5534281911,-1.7662620673,6.1680328922$

$\mathrm{H}, 0,0.0339214643,-3.3879965537,2.5526381619$

$\mathrm{H}, 0,-1.9502647885,-4.3431405863,3.7200089207$

C,0,-3.6036044002,-0.5152211061,-2.9694796109 
C, $0,-2.3543455612,-1.068438284,-3.696476094$ $\mathrm{H}, 0,-2.5533358595,-2.0693680911,-4.1014517093$ $\mathrm{H}, 0,-2.0862893155,-0.4149580225,-4.5362074693$ $\mathrm{H}, 0,-1.5003028933,-1.1228267495,-3.0205836489$ C,0,-4.7285430688,-0.3992208728,-4.0188061987 $\mathrm{H}, 0,-4.4021102892,0.2744693968,-4.8190644929$ H,0,-4.9696079204,-1.3644409694,-4.4811092309 $\mathrm{H}, 0,-5.6485368748,0.0195183152,-3.5942570129$ C, $0,-3.3196692478,0.9096013643,-2.4351085664$ $\mathrm{H}, 0,-4.2153482909,1.329160662,-1.9623182227$ $\mathrm{H}, 0,-2.5162071802,0.9103695115,-1.6983150767$ $\mathrm{H}, 0,-3.0342580653,1.5699684402,-3.2640914567$ C,0,1.6909862006,1.6900951271,-2.4763746568 C, $0,1.3098840506,0.3682158993,-3.1858384324$ $\mathrm{H}, 0,0.6221223118,-0.218907404,-2.5768533135$ $\mathrm{H}, 0,0.8258678201,0.5836697359,-4.1467894473$ $\mathrm{H}, 0,2.2044594283,-0.2350221973,-3.38689424$ C, $0,0.4286176059,2.5566214523,-2.2473303089$ $\mathrm{H}, 0,-0.2885350109,2.0579457897,-1.5950200759$ H, $0,0.6991617498,3.5171798844,-1.7923823035$ $\mathrm{H}, 0,-0.0604282774,2.7651554806,-3.2074588418$ C,0,2.6177201324,2.4715379013,-3.4305951183 $\mathrm{H}, 0,2.086045502,2.6581686776,-4.3705138831$ $\mathrm{H}, 0,2.9078753738,3.4465665923,-3.0214089405$ $\mathrm{H}, 0,3.5301761046,1.9141332676,-3.6756179312$ C, $0,-7.0744274095,-3.5621710025,-0.798175075$ C, $0,-7.1769708138,-4.4401758622,-2.0683088861$ $\mathrm{H}, 0,-8.1749788335,-4.8898995139,-2.145704423$ $\mathrm{H}, 0,-7.0031173432,-3.859014161,-2.980162796$ H,0,-6.4393933342,-5.2509236458,-2.0437997408 C, $0,-7.3773062573,-4.4507218864,0.4231067932$ $\mathrm{H}, 0,-6.6661050804,-5.2807771004,0.5092651585$ $\mathrm{H}, 0,-7.3518469633,-3.8785184795,1.3576308924$ $\mathrm{H}, 0,-8.3796927281,-4.8837861518,0.3275716868$ C, $0,-8.1473963349,-2.4486415135,-0.8594030841$ $\mathrm{H}, 0,-8.1059919399,-1.8169458416,0.0351034775$ $\mathrm{H}, 0,-8.0117917901,-1.7995785054,-1.7310628359$ $\mathrm{H}, 0,-9.1518021269,-2.8858669998,-0.9231960855$ C, $0,5.783910582,2.126953855,0.5393545708$ C, $0,6.7117273457,1.5859090531,-0.574362259$ $\mathrm{H}, 0,6.3602368843,1.8657842592,-1.5728013921$ $\mathrm{H}, 0,7.726529938,1.9864325162,-0.4567935996$ $\mathrm{H}, 0,6.7731800167,0.4916154632,-0.5410917862$ C, $0,6.3899983286,1.7327647141,1.8995757431$ $\mathrm{H}, 0,5.8050330227,2.1307618157,2.7365102231$ $\mathrm{H}, 0,6.4542971452,0.6438723477,2.0167170686$ 
$\mathrm{H}, 0,7.4055108494,2.1359979958,1.9861999537$

C, $0,5.7477137185,3.6726030146,0.45969903$

$\mathrm{H}, 0,5.3515467893,4.0192885221,-0.5006681205$

$\mathrm{H}, 0,5.1141770877,4.0893087716,1.2507962076$

$\mathrm{H}, 0,6.7567208172,4.0885452901,0.574413923$

$\mathrm{Cl}, 0,-2.0121393991,0.8968620168,1.7080214462$

$\mathrm{HF}=-2665.2358981$ (A.U.)

Zero-point correction $=1.028324$ (A.U.)

Total Gibbs free energy $=-2664.302740$ (A.U.)

model II

Cartesian Coordinates (Angstroms)

$\mathrm{C}, 0,3.537710527,2.4131915604,-1.0313105351$

C,0,2.2483910711,1.9712899856,-1.3087817987

C, $0,1.5009343323,1.3718961309,-0.2387195547$

C, $0,2.1631847679,1.1857918531,1.0163779291$

C,0,3.4833088028,1.661119392,1.2200738356

C,0,4.1920557741,2.2959051014,0.2194797127

$\mathrm{H}, 0,4.0935093429,2.8807492099,-1.8344878417$

$\mathrm{H}, 0,3.9231137772,1.5111665422,2.2021063395$

C, $0,1.5499636165,0.5122986402,2.1309359451$

$\mathrm{N}, 0,0.3922077457,-0.0669987521,2.1302621309$

$\mathrm{N}, 0,-1.6423523313,-1.6551726238,1.6313275704$

C, $0,-2.8264235213,-2.1366127367,1.4147952708$

C, $0,-3.6745553146,-1.8388756753,0.2913693665$

C, $0,-3.2536295257,-1.0219050713,-0.8064388471$

C, $0,-4.9584954306,-2.4394569884,0.3014198877$

C, $0,-4.168020863,-0.8411394774,-1.8990735221$

C, $0,-5.8539443266,-2.2630393197,-0.7333364284$

$\mathrm{H}, 0,-5.2180394498,-3.0517861471,1.1600783197$

C,0,-5.4119769961,-1.4582929617,-1.8118311278

$\mathrm{H}, 0,-6.1022571737,-1.3183049032,-2.6345191694$

$\mathrm{O}, 0,-2.0553511351,-0.4759773689,-0.8379564543$

$\mathrm{O}, 0,0.2441494858,1.0216905179,-0.4151285284$

Al, $0,-0.8371992364,-0.156103477,0.5142833013$

$\mathrm{H}, 0,2.1498926141,0.4931831441,3.0439358502$

H,0,-3.2466813288,-2.8484925232,2.1288955384

$\mathrm{P}, 0,-2.7301071167,4.9415785039,0.758344347$

C, $0,-3.5154928434,2.3817689237,2.6529299401$

$\mathrm{H}, 0,-3.0183942354,2.1985408707,3.612521512$

$\mathrm{H}, 0,-4.2757092041,1.6030514324,2.5300224078$

$\mathrm{H}, 0,-4.0097581687,3.3533497935,2.7046779251$

$\mathrm{O}, 0,-2.0013073747,1.0643700024,1.44894493$

$\mathrm{C}, 0,-2.4974772388,2.2467561333,1.534546465$

C,0,-2.1311111778,3.2966999868,0.7010959991 
$\mathrm{H}, 0,-1.3881924075,3.1100399182,-0.0673944129$ C,0,9.657746268,-3.4022950856,-0.9301148211 C, $0,8.6925236896,-4.2074927468,-1.5465725439$ C, $0,7.3423138034,-3.9021932137,-1.4241502496$ C, $0,6.9235102452,-2.7788105529,-0.6793801762$ C, $0,7.9099034897,-1.9795781672,-0.0660731476$ C, $0,9.2622307673,-2.2867900817,-0.1890258582$ H, $0,10.7117591935,-3.6451470198,-1.0293623683$ $\mathrm{H}, 0,8.99833682,-5.0747926107,-2.1245110037$ H,0,6.6082101261,-4.5360709272,-1.9119967079 C, $0,5.526330943,-2.4068422268,-0.5157934608$ H,0,7.6032633796,-1.1117526701,0.5129693022 $\mathrm{H}, 0,10.0064941675,-1.6582618685,0.2911842982$ C, $0,4.427232361,-3.0528917247,-0.9920906162$ C, $0,3.103469329,-2.5682305243,-0.7485989825$ C, $0,1.9592214275,-3.1617739065,-1.1955283894$ C, $0,0.6454606503,-2.6136702246,-0.9132800447$ $\mathrm{O}, 0,0.5220343927,-1.602665008,-0.1854175179$ C, $0,-0.5574323979,-3.281767631,-1.5304774863$ $\mathrm{H}, 0,5.3540458767,-1.4970094085,0.060181034$ $\mathrm{H}, 0,4.5287643585,-3.9665846884,-1.5739678994$ $\mathrm{H}, 0,3.0015440099,-1.6533861127,-0.1662444226$ $\mathrm{H}, 0,2.0085613415,-4.0660718949,-1.7960834826$ $\mathrm{H}, 0,-1.3755616596,-3.3422321583,-0.8092567163$ $\mathrm{H}, 0,-0.916201521,-2.6667403136,-2.3641685772$ $\mathrm{H}, 0,-0.3212432203,-4.2788147875,-1.9101074937$ C, $0,-0.8306077276,-2.0431737674,2.7968334378$ C, $0,-0.1590135829,-0.7437994087,3.3150663493$ C, $0,-1.5426057016,-2.7922032556,3.929496621$ C, $0,0.8091715161,-1.03957551,4.4662409818$ $\mathrm{H}, 0,-0.967323748,-0.097972819,3.6876663177$ C, $0,-0.5718210436,-3.0801677967,5.0867643221$ $\mathrm{H}, 0,-2.3864323724,-2.1888680173,4.2937806179$ C, $0,0.0949067588,-1.7968574589,5.5979793235$ $\mathrm{H}, 0,1.2300987842,-0.107267307,4.8617063338$ $\mathrm{H}, 0,1.6515093174,-1.6395350696,4.0924799446$ $\mathrm{H}, 0,-1.1089574571,-3.5799871022,5.9008637318$ $\mathrm{H}, 0,0.2015278441,-3.7829502551,4.7457004366$ $\mathrm{H}, 0,0.812127944,-2.0315927059,6.3928007884$ $\mathrm{H}, 0,-0.6665811085,-1.1439534093,6.0477909355$ $\mathrm{H}, 0,-0.0214619593,-2.6823648235,2.4110154954$ $\mathrm{H}, 0,-1.9586411997,-3.7375169805,3.5613394343$ C, $0,-3.7905247735,0.0098604289,-3.1316702386$ C, $0,-2.5425590416,-0.5839306134,-3.8285579301$ $\mathrm{H}, 0,-2.7340737334,-1.6140989561,-4.1537997295$ $\mathrm{H}, 0,-2.2930950526,0.0056306808,-4.7193170465$ 
$\mathrm{H}, 0,-1.6790646269,-0.576868417,-3.1623516464$ C, $0,-4.920232704,0.055759128,-4.1814869563$ $\mathrm{H}, 0,-4.5969219113,0.6707133624,-5.0286810303$ $\mathrm{H}, 0,-5.1634997959,-0.9382813367,-4.5743073794$ $\mathrm{H}, 0,-5.8387508081,0.5039173948,-3.7846796474$ C, $0,-3.5027671404,1.4679268743,-2.7000273901$ $\mathrm{H}, 0,-4.3997404759,1.9263969225,-2.2652863879$ $\mathrm{H}, 0,-2.6976395024,1.5132207005,-1.965729648$ $\mathrm{H}, 0,-3.2116802284,2.065302617,-3.5736724838$ C, $0,1.6588293179,2.0876728227,-2.7312830751$ C, $0,1.3026173507,0.6767082543,-3.2592824798$ $\mathrm{H}, 0,0.5634601482,0.1924912988,-2.6198515242$ $\mathrm{H}, 0,0.8889220086,0.7488203275,-4.2727754623$ $\mathrm{H}, 0,2.1961956975,0.0424694519,-3.3052629217$ C, $0,0.3948488862,2.9778499368,-2.7247376946$ $\mathrm{H}, 0,-0.3853036851,2.5482864559,-2.095285059$ $\mathrm{H}, 0,0.6295187875,3.9843375141,-2.3597094213$ $\mathrm{H}, 0,0.0001924021,3.0741884963,-3.7442140542$ C, $0,2.6553735901,2.7136093854,-3.7288986915$ H, $0,2.1903541869,2.7563973965,-4.7199661394$ $\mathrm{H}, 0,2.9311224576,3.738461113,-3.4534170961$ $\mathrm{H}, 0,3.5737943495,2.1228940519,-3.8248189145$ C, $0,-7.2579118717,-2.8911442332,-0.7670994929$ C, $0,-7.3849762162,-3.8151934721,-2.0019851419$ $\mathrm{H}, 0,-8.3835414312,-4.2667211465,-2.0397108042$ $\mathrm{H}, 0,-7.2333446421,-3.2693337841,-2.9393490051$ $\mathrm{H}, 0,-6.6472145258,-4.6247406458,-1.9631859944$ C, $0,-7.5450653254,-3.7309254676,0.4918139246$ $\mathrm{H}, 0,-6.839729916,-4.5636753441,0.5956371305$ $\mathrm{H}, 0,-7.5002312105,-3.125962902,1.4050646876$ $\mathrm{H}, 0,-8.5515786876,-4.1588556806,0.4301370644$ C, $0,-8.3251998056,-1.7738903744,-0.8536861014$ $\mathrm{H}, 0,-8.2725791011,-1.1130825219,0.0195565988$ H, $0,-8.1994479619,-1.1566868441,-1.7499396589$ H,0,-9.3314240907,-2.2084629789,-0.8901700686 C, $0,5.6087179046,2.8674479565,0.4007355531$ C, $0,6.5747633869,2.2292840235,-0.6253785097$ H, $0,6.2640524108,2.4194861837,-1.6579171127$ $\mathrm{H}, 0,7.5849636254,2.6384143149,-0.504484347$ $\mathrm{H}, 0,6.6313028885,1.1424073053,-0.4917855146$ C, $0,6.1648798327,2.6005459565,1.8123308157$ $\mathrm{H}, 0,5.5469389696,3.0648137859,2.5896616916$ $\mathrm{H}, 0,6.235675011,1.5274118088,2.0279833536$ $\mathrm{H}, 0,7.172652255,3.0212731669,1.9000857141$ C,0,5.5741731377,4.3991628858,0.1791842663 $\mathrm{H}, 0,5.2115419363,4.6566036158,-0.8217698559$ 
$\mathrm{H}, 0,4.9159071748,4.8848754405,0.9087363363$

$\mathrm{H}, 0,6.5784156364,4.8260372775,0.2894368641$

C, $0,-1.7253513504,5.9533715934,-0.3785711589$

C, $0,-0.7914623679,6.8854850225,0.0954306101$

C, $0,-1.8992543129,5.783251948,-1.7626383675$

C, $0,-0.0377463929,7.6375936788,-0.8074591643$

$\mathrm{H}, 0,-0.6532929098,7.0309420877,1.1615364463$

C, $0,-1.1426618544,6.5379775738,-2.657518728$

$\mathrm{H}, 0,-2.6237183972,5.0687920736,-2.1422534112$

C,0,-0.2126539699,7.4659576949,-2.1812160576

$\mathrm{H}, 0,0.681532743,8.3606761633,-0.4343229486$

$\mathrm{H}, 0,-1.2815252536,6.4026855597,-3.7259636526$

$\mathrm{H}, 0,0.3717608334,8.0562396905,-2.8811368842$

C, $0,-2.5919523919,5.6645557712,2.434822555$

C, $, 0,-3.5605123273,6.527136399,2.9693460162$

C, $0,-1.453551868,5.3455706582,3.1956988512$

C, $0,-3.3874614184,7.068502854,4.2444033601$

$\mathrm{H}, 0,-4.4545703103,6.7673204728,2.4044807118$

C, $0,-1.2833011739,5.8968446528,4.4651204571$

$\mathrm{H}, 0,-0.7124291845,4.6591952047,2.7964918149$

C, $0,-2.2496097844,6.7583267838,4.9908685196$

$\mathrm{H}, 0,-4.1454724448,7.7297718274,4.6537731754$

$\mathrm{H}, 0,-0.3998178292,5.64841387,5.0461055491$

$\mathrm{H}, 0,-2.1182963367,7.1818250593,5.9824458977$

C,0,-4.4562821943,5.1451979924,0.1842284113

C, $0,-4.9544145897,6.4221946742,-0.1315992612$

C, $0,-5.2807572056,4.022983808,0.024344855$

C, $0,-6.2681701062,6.570135388,-0.5736946407$

$\mathrm{H}, 0,-4.3156055631,7.2971728326,-0.0496374642$

C,0,-6.5956316802,4.1777826526,-0.4194150674

$\mathrm{H}, 0,-4.88968198,3.0310396208,0.2241344963$

C, $0,-7.0910379374,5.4488008119,-0.7133544933$

$\mathrm{H}, 0,-6.6463523974,7.5585747777,-0.8176519462$

$\mathrm{H}, 0,-7.2274492121,3.3030889391,-0.5423708366$

$\mathrm{H}, 0,-8.1129411917,5.5668459498,-1.0619942033$

$\mathrm{HF}=-3433.0867804$ (A.U.)

Zero-point correction $=1.370447$ (A.U.)

Total Gibbs free energy $=-3431.837237$ (A.U.)

TS1

Cartesian Coordinates (Angstroms)

C, $0,1.6797611046,3.9434712632,0.526035392$

C, $0,0.5801388136,3.2924628503,-0.0179453811$

C, $0,0.1946419355,2.0192871931,0.5459158623$

C, $0,0.9461877099,1.5373877118,1.6690904752$ 
C,0,2.0396497755,2.2762745578,2.1816874904 C,0,2.4456721655,3.4730539291,1.6239728121 $\mathrm{H}, 0,1.9646957903,4.8967383114,0.0960394723$ $\mathrm{H}, 0,2.5475583004,1.8701065509,3.0531399013$ C, $0,0.5957775693,0.3334700499,2.3826077248$ $\mathrm{N}, 0,-0.3145437859,-0.5170204653,2.0471832049$ $\mathrm{N}, 0,-1.9086685329,-2.2816785725,0.8976355171$ C, $0,-2.9377069063,-2.9246081874,0.4462614322$ C, $0,-3.8306236687,-2.4796508347,-0.5913558813$ C, $0,-3.6197048228,-1.2732451273,-1.3325112997$ C, $0,-4.9302059625,-3.324112704,-0.8777388026$ C, $0,-4.5606396728,-0.9487984526,-2.3698553687$ C, $0,-5.8427279034,-3.0245135232,-1.8688242095$ $\mathrm{H}, 0,-5.0354944638,-4.2257027358,-0.2808339186$ C, $0,-5.6157942753,-1.8256427592,-2.5890648856$ $\mathrm{H}, 0,-6.3267564149,-1.575828487,-3.3677260929$ $\mathrm{O}, 0,-2.5867757647,-0.4980536118,-1.1086400416$ $\mathrm{O}, 0,-0.8104105249,1.3630102775,0.0476353773$ Al, $0,-1.4971153334,-0.3448870619,0.385835857$ $\mathrm{H}, 0,1.1627440964,0.166978256,3.3034938986$ $\mathrm{H}, 0,-3.1737456907,-3.9072585388,0.8620113643$ C, $0,7.3810422031,3.3709844348,-4.6616525557$ $\mathrm{C}, 0,6.8323913309,2.1906279714,-5.174787651$ C,0,5.7176176555,1.6120126637,-4.5760433451 C,0,5.1168892535,2.1984609471,-3.4426400047 C, $0,5.6801882667,3.3897087484,-2.9436854183$ C, $0,6.7975372995,3.9686289285,-3.5433578652$ $\mathrm{H}, 0,8.2493068684,3.8214413737,-5.1344801511$ $\mathrm{H}, 0,7.2733823335,1.7235849696,-6.0516058435$ $\mathrm{H}, 0,5.3004875114,0.7036425988,-5.0014193252$ C,0,3.9505778224,1.6309154104,-2.7610912158 $\mathrm{H}, 0,5.2263592638,3.8646970261,-2.0769840814$ H,0,7.209083638,4.8897217139,-3.1392650123 C, $0,3.3297663165,0.4546541116,-3.025541321$ C,0,2.2081101226,-0.0464382828,-2.2596770832 C,0,1.6661765109,-1.2697420558,-2.4167795965 C, $0,0.5203979818,-1.7583523276,-1.5756387778$ $\mathrm{O}, 0,0.1293771171,-0.9831349668,-0.6447103612$ C, $0,-0.4728492511,-2.6387531564,-2.3202223517$ $\mathrm{H}, 0,3.5486391255,2.2374571712,-1.9481947827$ $\mathrm{H}, 0,3.6866514101,-0.1777887185,-3.8396568313$ $\mathrm{H}, 0,1.7912252147,0.5971772219,-1.4874419866$ $\mathrm{H}, 0,2.0321964068,-1.9528591536,-3.1819170451$ $\mathrm{H}, 0,-1.123763521,-3.1664141644,-1.6243552455$ $\mathrm{H}, 0,-1.0954876549,-1.9874273353,-2.9433893294$ $\mathrm{H}, 0,0.0404333744,-3.3644491837,-2.9572616217$ 
C,0,-1.0197411879,-2.8296256522,1.9323626788

C, $0,-0.6898901974,-1.6588556396,2.8945223677$

C, $0,-1.5266599263,-4.053496001,2.7057157598$

C, $0,0.31624205,-2.0836112983,3.9707925735$

$\mathrm{H}, 0,-1.6320984382,-1.3637319087,3.3773767178$

C, $0,-0.5146576424,-4.4754889546,3.7830119935$

$\mathrm{H}, 0,-2.4921430575,-3.8165534323,3.1748665278$

C, $0,-0.189810796,-3.3175025074,4.7350645606$

$\mathrm{H}, 0,0.4859927993,-1.2639436575,4.6796010512$

H,0,1.284069796,-2.3115848601,3.5003476795

$\mathrm{H}, 0,-0.9085984282,-5.3308785627,4.3456532133$

$\mathrm{H}, 0,0.4096852212,-4.8180797632,3.2961046961$

$\mathrm{H}, 0,0.5597511353,-3.6291989885,5.4732832606$

$\mathrm{H}, 0,-1.0924135348,-3.0466173861,5.3006015954$

$\mathrm{H}, 0,-0.0836265833,-3.1019725263,1.4220136635$

$\mathrm{H}, 0,-1.6974614786,-4.8930969844,2.0212361893$

C, $0,-4.4138407076,0.3485863643,-3.1919637752$

C, $0,-3.0637885606,0.3529175973,-3.9499341793$

$\mathrm{H}, 0,-3.0055828,-0.4976261399,-4.6418897066$

$\mathrm{H}, 0,-2.9676183657,1.272315659,-4.5417888221$

$\mathrm{H}, 0,-2.2228436861,0.2999462491,-3.2576229337$

C, $0,-5.5282295667,0.5020836471,-4.2480114943$

$\mathrm{H}, 0,-5.3729964378,1.4375469732,-4.7978319371$

$\mathrm{H}, 0,-5.5230492959,-0.3143287417,-4.9808194368$

$\mathrm{H}, 0,-6.5249788954,0.5520753755,-3.7940795013$

C, $0,-4.4976050424,1.5733256325,-2.2487025294$

$\mathrm{H}, 0,-5.4769782874,1.6141987606,-1.7574246039$

$\mathrm{H}, 0,-3.7324033999,1.5351290551,-1.4730320741$

$\mathrm{H}, 0,-4.3691381665,2.4995234151,-2.8242490189$

C, $0,-0.2405221504,3.9217376643,-1.1632343182$

C, $0,-0.248111075,2.9947006225,-2.402663572$

$\mathrm{H}, 0,-0.6705606467,2.0182995679,-2.1651107651$

$\mathrm{H}, 0,-0.8483893776,3.447355056,-3.2020516275$

$\mathrm{H}, 0,0.7690147726,2.8533149634,-2.790528269$

C,0,-1.691953427,4.1647835287,-0.6775662629

$\mathrm{H}, 0,-2.1665547192,3.2390458149,-0.3499811962$

$\mathrm{H}, 0,-1.7045087527,4.8735359608,0.1595265897$

$\mathrm{H}, 0,-2.2902189969,4.5940525708,-1.4914233671$

C, $0,0.3287828384,5.2831135471,-1.6127818974$

$\mathrm{H}, 0,-0.295808266,5.6823169464,-2.4198846538$

$\mathrm{H}, 0,0.3272780112,6.0223180607,-0.8027674204$

$\mathrm{H}, 0,1.3512550652,5.1999285442,-2.002583325$

C,0,-7.0553239495,-3.9067571349,-2.2105121804

C, $0,-6.9599434989,-4.3796731833,-3.6808494719$

H,0,-7.8247118902,-5.0027942226,-3.9430297406

$\mathrm{H}, 0,-6.9349318059,-3.5361021774,-4.3790546138$ 
$\mathrm{H}, 0,-6.0517465518,-4.9722109282,-3.8420872607$

C, $0,-7.1341726322,-5.15612713,-1.3122983706$

$\mathrm{H}, 0,-6.2492660801,-5.7937481863,-1.4242413647$

$\mathrm{H}, 0,-7.2321906885,-4.8896042767,-0.2535360899$

$\mathrm{H}, 0,-8.0104161614,-5.7556705324,-1.5853889791$

C, $0,-8.3600172447,-3.0969371539,-2.0187290628$

$\mathrm{H}, 0,-8.4588457294,-2.7575055745,-0.9814327993$

$\mathrm{H}, 0,-8.3869866462,-2.2109887402,-2.6619500215$

$\mathrm{H}, 0,-9.2354644006,-3.7124205768,-2.262704104$

C,0,3.6220727073,4.3095166074,2.1551502861

C,0,4.6612517263,4.5336520711,1.0302914581

$\mathrm{H}, 0,4.2225639376,5.05324151,0.1715885018$

$\mathrm{H}, 0,5.5009478236,5.1408579544,1.3919061247$

$\mathrm{H}, 0,5.063798352,3.5772502554,0.6727801453$

C, $0,4.3390843217,3.6221846334,3.3327034131$

$\mathrm{H}, 0,3.668137589,3.4749971327,4.186330333$

$\mathrm{H}, 0,4.7444599951,2.64354319,3.0460858696$

$\mathrm{H}, 0,5.1767684584,4.2415388756,3.6738649125$

C,0,3.10721229,5.6838881697,2.6468341968

$\mathrm{H}, 0,2.6149433664,6.2426894431,1.8441301216$

$\mathrm{H}, 0,2.3805366845,5.5580950699,3.4570940057$

$\mathrm{H}, 0,3.9362436368,6.2977574493,3.0222277917$

$\mathrm{Si}, 0,5.6232824263,-4.1443937396,2.1038365684$

C, $0,1.6570686463,-3.1872985375,-0.682775665$

N,0,2.4821055761,-3.6525404774,0.0125997388

$\mathrm{P}, 0,5.4127358011,-1.5303800984,0.3012494817$

O, $0,5.9235743376,-2.6034511822,1.3360428612$

C, $0,5.1848162152,-2.2673246266,-1.3300984472$

$\mathrm{H}, 0,4.3464580926,-2.9691618697,-1.2783214366$

$\mathrm{H}, 0,4.949647919,-1.4742915968,-2.0464858961$

$\mathrm{H}, 0,6.1035083653,-2.7803785977,-1.6300478159$

C, $0,3.9029583613,-0.7288684836,0.8721668539$

$\mathrm{H}, 0,3.6579942389,0.1082041666,0.2123389987$

H, $0,3.0788015774,-1.4479837115,0.8470953157$

$\mathrm{H}, 0,4.0551311873,-0.3497293383,1.8868416597$

C, $0,6.72740767,-0.2857052986,0.1934117442$

$\mathrm{H}, 0,7.6601963049,-0.7581117237,-0.1279846785$

H, 0,6.4448209931,0.4854454894,-0.5310185377

$\mathrm{H}, 0,6.8814075452,0.17766503,1.1723160964$

C,0,7.1846289858,-4.3807927951,3.1193006677

$\mathrm{H}, 0,7.1369471054,-5.3241819051,3.677207894$

$\mathrm{H}, 0,8.0769386933,-4.4166661452,2.4839620463$

H,0,7.3188922835,-3.5720493299,3.8465771306

C, $0,5.4235705812,-5.4325037866,0.76005082$

$\mathrm{H}, 0,5.3568195155,-6.4315461228,1.2096867341$

H,0,4.4958090795,-5.2517715296,0.2066101485 
$\mathrm{H}, 0,6.2690459063,-5.4434698598,0.0622398615$

C,0,4.0858911097,-3.960523946,3.1541426198

$\mathrm{H}, 0,3.2143515321,-3.8218339464,2.505116872$

$\mathrm{H}, 0,3.9235741268,-4.8694602791,3.7473875877$

H,0,4.1574785534,-3.118676597,3.8525385249

$\mathrm{Cl}, 0,-3.2335237425,0.4286171163,1.8105534724$

only imaginary frequency: $-237.5 \mathrm{~cm}^{-1}$

$\mathrm{HF}=-3703.6753449$ (A.U.)

Zero-point correction $=1.269500$ (A.U.)

Total Gibbs free energy $=-3702.521750$ (A.U.)

\section{IM1}

Cartesian Coordinates (Angstroms)

C, $0,-2.5543971227,-2.6181590178,2.4898007003$

C, $0,-1.2940870818,-2.5884797538,1.9081852567$

C, $0,-0.6163860729,-1.3133445426,1.8166095267$

C, $0,-1.2308070751,-0.1897603351,2.4649515346$

C, $0,-2.5031200344,-0.3136376293,3.0744115211$

C, $0,-3.210026134,-1.5011817003,3.0710489938$

$\mathrm{H}, 0,-3.0658510264,-3.572838331,2.5370669979$

$\mathrm{H}, 0,-2.9050914494,0.5682761867,3.568147557$

C,0,-0.5607684091,1.0796420473,2.6250551097

$\mathrm{N}, 0,0.564339501,1.423600105,2.0966993076$

$\mathrm{N}, 0,2.7424674431,1.9588588235,0.6770736615$

C, $0,3.9732836365,1.9949080162,0.278295211$

C, $0,4.721885332,0.8930069141,-0.2678807751$

C, $0,4.1452816156,-0.3948460494,-0.5146253812$

C, $0,6.0764925807,1.152880862,-0.5862414596$

C, $0,4.9888897057,-1.4093728717,-1.0873880184$

C, $0,6.8974554539,0.1889832924,-1.1358361504$

$\mathrm{H}, 0,6.4550939318,2.1495124357,-0.3768328034$

C,0,6.3086237922,-1.0787915622,-1.3674466693

H,0,6.9428072173,-1.8477017327,-1.7928048698

$\mathrm{O}, 0,2.8839129873,-0.6452744539,-0.2717317396$

O, $0,0.5140772808,-1.2226685919,1.1878453406$

Al, $0,1.6638793526,0.224184663,0.8346488592$

$\mathrm{H}, 0,-1.070926933,1.7786821041,3.2956810648$

$\mathrm{H}, 0,4.5262985193,2.9360244407,0.3391807487$

C, $0,-6.3456725651,-4.5287957631,-4.0941307173$

C, $0,-5.474842882,-3.8083657258,-4.9187775318$

C, $0,-4.4149817859,-3.0918510931,-4.37148128$

C, $0,-4.1939739962,-3.074751628,-2.9788780736$

C, $0,-5.0783431161,-3.8101532435,-2.1651444807$

C, $0,-6.140493756,-4.5273146831,-2.7135050896$

$\mathrm{H}, 0,-7.1698539332,-5.0896343108,-4.5260477176$ 
$\mathrm{H}, 0,-5.620755629,-3.8112945384,-5.9958893388$

$\mathrm{H}, 0,-3.7443018492,-2.5505872656,-5.032798382$

C, $0,-3.1051753496,-2.3293085869,-2.3409972982$

$\mathrm{H}, 0,-4.9188263837,-3.8207640083,-1.0890899032$

$\mathrm{H}, 0,-6.8044460125,-5.0893456532,-2.0620364848$

C, $0,-2.204141736,-1.5076967033,-2.9323815916$

C, $0,-1.2122147374,-0.7465888516,-2.1960391801$

C, $0,-0.4732674994,0.2415361572,-2.727968874$

C, $0,0.4542240949,1.1309504325,-1.8847440084$

O, $0,0.4625837462,0.7906061543,-0.5706083496$

C, $0,1.8248382735,1.2387266774,-2.5973091866$

$\mathrm{H}, 0,-3.0276774538,-2.4619741235,-1.2611815805$

H,0,-2.234419406,-1.3544069714,-4.0121148234

H, $0,-1.0840595131,-0.9475267434,-1.1343721482$

$\mathrm{H}, 0,-0.5420015881,0.4836025379,-3.7901369798$

$\mathrm{H}, 0,2.4721353147,1.9454926317,-2.0753214089$

$\mathrm{H}, 0,2.2995151533,0.2569584411,-2.5853855532$

H,0,1.7041732599, 1.5699591146,-3.6357642394

C,0,2.0377727939,3.134243985,1.20161714

C, $0,1.2641615674,2.6643773948,2.4623718961$

C, $0,2.8863742817,4.3754672273,1.5040850916$

C, $0,0.4079901446,3.7952931073,3.0466404413$

$\mathrm{H}, 0,2.0181303646,2.3606180977,3.2021964416$

C,0,2.02122098,5.504459959,2.0849321121

$\mathrm{H}, 0,3.6786733374,4.1113052118,2.2185986975$

C, $0,1.2614303693,5.0408430353,3.3340720084$

$\mathrm{H}, 0,-0.0733830405,3.4690964224,3.9761358733$

$\mathrm{H}, 0,-0.3956242486,4.0503903985,2.3396544242$

$\mathrm{H}, 0,2.6515193504,6.3704823343,2.3218214714$

$\mathrm{H}, 0,1.3017792998,5.8393794623,1.3232922974$

H,0,0.6236261219,5.8481474888,3.7157259595

$\mathrm{H}, 0,1.9817747921,4.8078348744,4.1307513207$

$\mathrm{H}, 0,1.2833054632,3.4035881545,0.4462005273$

$\mathrm{H}, 0,3.3819601489,4.7286159991,0.5914342035$

C, $0,4.4447704003,-2.8283441207,-1.35641656$

C, $0,3.2624613109,-2.7710922819,-2.355155321$

$\mathrm{H}, 0,3.5843225353,-2.3405648752,-3.3126189571$

$\mathrm{H}, 0,2.8891907312,-3.784328273,-2.5532266401$

H,0,2.4402033256,-2.172854166,-1.9606720899

C, $0,5.5119675756,-3.7583230348,-1.9700203378$

$\mathrm{H}, 0,5.0705401722,-4.7476373649,-2.1373316875$

$\mathrm{H}, 0,5.8755250831,-3.3932054873,-2.9386615498$

H,0,6.3748092519,-3.8937992806,-1.3071925111

C, $0,3.9808110165,-3.4683608788,-0.0255988108$

$\mathrm{H}, 0,4.8237532813,-3.5674087868,0.6683296488$

$\mathrm{H}, 0,3.2124318967,-2.867323256,0.4608074958$ 
$\mathrm{H}, 0,3.576918736,-4.472114518,-0.2136966911$ $\mathrm{C}, 0,-0.5833448132,-3.8844863673,1.4602798586$ C, $0,-0.1961779811,-3.8424140721,-0.0368164826$ $\mathrm{H}, 0,0.4534911102,-2.9959415077,-0.2569058127$ $\mathrm{H}, 0,0.3331505976,-4.7650550962,-0.3066482056$ $\mathrm{H}, 0,-1.0884449083,-3.7790264499,-0.6721916685$ C, $0,0.692053559,-4.0650555458,2.3232396647$ $\mathrm{H}, 0,1.3702725926,-3.2156846741,2.2247519949$ $\mathrm{H}, 0,0.4277386998,-4.1721997346,3.3823006488$ H,0,1.2253394766,-4.9732918646,2.0145404233 C, $0,-1.4640261765,-5.1345357919,1.6670482447$ $\mathrm{H}, 0,-0.9066523443,-6.0214738161,1.3451445199$ $\mathrm{H}, 0,-1.7355071615,-5.2839548411,2.7188363153$ $\mathrm{H}, 0,-2.3871200647,-5.0966835593,1.0739720241$ C, $0,8.3756892669,0.424017853,-1.4896761338$ C, $0,8.5978255913,0.1755199372,-3.000969299$ $\mathrm{H}, 0,9.6521621807,0.3263327985,-3.2666613661$ $\mathrm{H}, 0,8.3252907352,-0.8450995816,-3.2902543012$ $\mathrm{H}, 0,7.9929242823,0.8642406549,-3.602464709$ C, $0,8.8261042128,1.862690266,-1.1730870257$ $\mathrm{H}, 0,8.2494839817,2.6029707392,-1.7403193992$ $\mathrm{H}, 0,8.7248179752,2.0940117172,-0.1064722765$ $\mathrm{H}, 0,9.8817224139,1.9898276025,-1.440663569$ C,0,9.2667310396,-0.546015576,-0.6772514702 $\mathrm{H}, 0,9.1452419118,-0.376785814,0.3986361747$ $\mathrm{H}, 0,9.0164202836,-1.5930853121,-0.8784138449$ $\mathrm{H}, 0,10.3252323633,-0.4014885743,-0.9297783072$ C, $0,-4.6030547538,-1.6702333249,3.7013446941$ C, $0,-5.605491701,-2.1642548018,2.6307174139$ H,0,-5.2901747251,-3.1146047079,2.1870745992 H,0,-6.6010614162,-2.3140961819,3.0678158221 $\mathrm{H}, 0,-5.6996816164,-1.4328093663,1.8173737071$ C, $0,-5.142763631,-0.349890231,4.2832004955$ $\mathrm{H}, 0,-4.4994804824,0.0346161384,5.0825414837$ $\mathrm{H}, 0,-5.2304782434,0.4287466373,3.5146571822$ H,0,-6.1405929428,-0.50819749662,4.7088084023 C, $0,-4.5406859685,-2.705725649,4.8501973118$ $\mathrm{H}, 0,-4.199730988,-3.6841879474,4.4964704583$ $\mathrm{H}, 0,-3.8473208645,-2.3758405886,5.631698301$ $\mathrm{H}, 0,-5.5306008189,-2.8413134988,5.3046657243$ $\mathrm{Si}, 0,-4.2628360737,5.0338720572,-0.5694931478$ C, $0,-0.2304240706,2.4961759942,-1.9815171956$ $\mathrm{N}, 0,-0.8540675861,3.4813651973,-1.9674128443$ $\mathrm{P}, 0,-4.3757199407,1.9257279266,-1.233036176$ $\mathrm{O}, 0,-4.7903161001,3.3868291514,-0.8085740213$ C,0,-3.7747171091,1.9146365126,-2.9364906224 
$\mathrm{H}, 0,-2.8612419582,2.5129224987,-2.9988166863$

$\mathrm{H}, 0,-3.5485834106,0.884235352,-3.2277712456$

$\mathrm{H}, 0,-4.5437678853,2.3295989684,-3.5950469555$

C, $0,-3.1717183227,1.2157527567,-0.0985880658$

$\mathrm{H}, 0,-3.0484671796,0.1550397812,-0.3330416946$

$\mathrm{H}, 0,-2.1917937909,1.6918952746,-0.1876763415$

H,0,-3.5402124988, 1.2999072965,0.9274056562

C, $0,-5.8902190189,0.9334741942,-1.1517722333$

$\mathrm{H}, 0,-6.6555520882,1.3682336517,-1.8011347607$

$\mathrm{H}, 0,-5.6763468636,-0.0898628011,-1.4779450554$

$\mathrm{H}, 0,-6.2631324948,0.9116837992,-0.1235209015$

C, $0,-5.8232289067,5.8632983637,0.0596583449$

$\mathrm{H}, 0,-5.637436265,6.9262749345,0.2567743098$

$\mathrm{H}, 0,-6.6357598612,5.7990756363,-0.6728962013$

$\mathrm{H}, 0,-6.1723410516,5.4086296017,0.993585635$

C, $0,-3.6971761889,5.6990260442,-2.2264594685$

$\mathrm{H}, 0,-3.4870796901,6.772949178,-2.1410612415$

$\mathrm{H}, 0,-2.7712393842,5.20758539,-2.5441970098$

$\mathrm{H}, 0,-4.4585007539,5.5759249443,-3.0055108789$

C, $0,-2.8828495293,5.0095553996,0.6953695749$

$\mathrm{H}, 0,-1.9922503603,4.5207451517,0.2853274646$

$\mathrm{H}, 0,-2.602558219,6.0370653427,0.9601361371$

H,0,-3.1765764007,4.4999029419,1.6200977553

$\mathrm{Cl}, 0,2.9785566164,-0.3006329033,2.8155603984$

$\mathrm{HF}=-3703.6868084$ (A.U.)

Zero-point correction $=1.270690$ (A.U.)

Total Gibbs free energy $=-3702.532750$ (A.U.)

\section{TS2}

Cartesian Coordinates (Angstroms)

$\mathrm{C}, 0,-0.8448850482,-3.8978701455,-1.0408266472$

C, $0,-0.1662574524,-2.7479896571,-1.4337965914$

C, $0,-0.0245755214,-1.6880350641,-0.4694382082$

C, $0,-0.6273725,-1.8714218959,0.8170426262$

C, $0,-1.2915955712,-3.0778070357,1.1439945108$

C, $0,-1.4136508786,-4.1148737218,0.239341255$

$\mathrm{H}, 0,-0.9462210979,-4.6946258766,-1.7680235514$

$\mathrm{H}, 0,-1.7061203821,-3.1634685837,2.1446885914$

C, $0,-0.577094948,-0.8700009824,1.8539343409$

$\mathrm{N}, 0,-0.0947903519,0.3234285052,1.741063772$

$\mathrm{N}, 0,0.4345359252,2.8169106177,1.0645555158$

C,0,1.059614401,3.9121562054,0.7694417981

C, $0,1.8614986642,4.1452791624,-0.4031225971$

C,0,1.9920536426,3.1899511333,-1.4618319373

C, $0,2.5057908763,5.4048536621,-0.4785540851$ 
C, $0,2.7985323397,3.5501948847,-2.5946369088$

C, $0,3.2877630864,5.7631137013,-1.5578467317$

$\mathrm{H}, 0,2.3626549985,6.0893711479,0.3527074468$

C, $0,3.4043334474,4.801949212,-2.5914088674$

$\mathrm{H}, 0,4.0146743676,5.0688074523,-3.445469875$

$\mathrm{O}, 0,1.3753089239,2.0296651923,-1.4263688262$

$\mathrm{O}, 0,0.6648602097,-0.6108202332,-0.7571278381$

Al,0,0.6432991518,1.0978277249,-0.0027118691

$\mathrm{H}, 0,-0.9963399332,-1.1845766417,2.8134143501$

$\mathrm{H}, 0,0.9847469261,4.7643404721,1.448947625$

$\mathrm{P}, 0,4.8843118842,-2.1564639828,0.9704381586$

C, $0,4.6382054954,1.0698385501,1.6163546739$

$\mathrm{H}, 0,4.294538885,1.6231948017,2.4969134453$

$\mathrm{H}, 0,4.9581330052,1.8136060459,0.8780978202$

$\mathrm{H}, 0,5.503191731,0.4638012087,1.8937883056$

O, $0,2.4350037892,1.0048535284,0.8244908408$

C,0,3.4712321605,0.285928242,1.0405813833

C, $0,3.5272018998,-1.0801056151,0.7676766967$

$\mathrm{H}, 0,2.6550429788,-1.5364339664,0.3142091621$

C, $0,-8.2615947223,-3.9364153308,-4.2127003436$

C, $0,-8.1085907901,-2.5664027474,-4.4519123455$

C,0,-7.0264933604,-1.8739427416,-3.9169711349

C, $0,-6.0627197853,-2.5342073694,-3.1257167469$

C, $0,-6.2333569764,-3.9138889812,-2.8980679372$

C,0,-7.3172234812,-4.6071532852,-3.4337920897

$\mathrm{H}, 0,-9.105761382,-4.473908522,-4.6349215592$

$\mathrm{H}, 0,-8.8339059307,-2.0384104087,-5.0651386211$

$\mathrm{H}, 0,-6.9217007959,-0.8136325773,-4.1295030495$

C, $0,-4.9081646017,-1.8586349087,-2.5282409274$

$\mathrm{H}, 0,-5.4979108516,-4.4447575939,-2.2984405907$

H,0,-7.4220419177,-5.6722085611,-3.2461408235

C, $0,-4.5843969199,-0.5447130997,-2.6147364452$

C, $0,-3.4476646102,0.0545846886,-1.9495413481$

C, $0,-3.2025593292,1.380022855,-1.9433586156$

C, $0,-2.0442970965,2.0075193265,-1.2370268756$

$\mathrm{O}, 0,-1.2343450256,1.2257539663,-0.6388714202$

C, $0,-1.5535691131,3.3076528868,-1.8495223836$

$\mathrm{H}, 0,-4.2419127981,-2.5019560983,-1.9518991277$

$\mathrm{H}, 0,-5.2067426266,0.1278810388,-3.2063671378$

$\mathrm{H}, 0,-2.7711644795,-0.5985121867,-1.4013887531$

$\mathrm{H}, 0,-3.8567498313,2.0656905812,-2.4791400054$

$\mathrm{H}, 0,-0.9220750546,3.8589652518,-1.1549674309$

$\mathrm{H}, 0,-0.9525904788,3.0559940198,-2.7312401134$

$\mathrm{H}, 0,-2.3900847574,3.9405735168,-2.1559824687$

C, $0,-0.386584382,2.6701125349,2.2751377667$

C, $0,-0.0543575581,1.2738108327,2.8650616987$ 
C, $0,-0.2613449621,3.7652444197,3.3405366077$ C, $0,-0.9414387618,0.9579894913,4.0750856749$ $\mathrm{H}, 0,0.9953497503,1.3079223771,3.1936134592$ C, $0,-1.1484702236,3.4446587181,4.5540959164$ $\mathrm{H}, 0,0.7874448851,3.855925253,3.6594022031$ C, $0,-0.8278436106,2.0629650641,5.1383934715$ H,0,-0.6577669527,-0.0036609148,4.5200859818 $\mathrm{H}, 0,-1.985923211,0.8663365789,3.7443744161$ $\mathrm{H}, 0,-1.0208985455,4.218933416,5.3198988771$ $\mathrm{H}, 0,-2.2030795172,3.4761777754,4.2469084173$ H,0,-1.4975693111,1.8380098394,5.9770096349 $\mathrm{H}, 0,0.1928047244,2.0678843786,5.5471421155$ $\mathrm{H}, 0,-1.4314776514,2.6363842547,1.9362528913$ $\mathrm{H}, 0,-0.5562380371,4.735036175,2.9229962876$ C, $0,2.9961963168,2.5736509653,-3.7738415675$ C, $0,1.6332077137,2.2419865974,-4.4283028515$ $\mathrm{H}, 0,1.1575012473,3.154151116,-4.8094576606$ $\mathrm{H}, 0,1.778150234,1.5614612477,-5.2768880682$ $\mathrm{H}, 0,0.958307042,1.7658051177,-3.7161881635$ C, $0,3.9020247935,3.159523454,-4.8767935206$ H,0,4.0099340352,2.4233639974,-5.6814269233 $\mathrm{H}, 0,3.4799470143,4.06841637,-5.321233761$ H, $0,4.9084837543,3.3920891179,-4.5088004864$ C, $0,3.667288455,1.2728748577,-3.2684623638$ H,0,4.6584743956, 1.4935632746,-2.8505368212 $\mathrm{H}, 0,3.0623567385,0.7910998887,-2.4988470951$ $\mathrm{H}, 0,3.7999333831,0.5692802256,-4.1002809502$ C, $0,0.4037762704,-2.6073104972,-2.8627062311$ C, $0,-0.2362661666,-1.38576114,-3.5659307885$ $\mathrm{H}, 0,-0.0427320123,-0.4635155858,-3.0173476756$ $\mathrm{H}, 0,0.1755302136,-1.2802383934,-4.5773449229$ $\mathrm{H}, 0,-1.3212939245,-1.5151276794,-3.6587347701$ C, $0,1.9414738352,-2.4365966933,-2.8160637285$ $\mathrm{H}, 0,2.2248319643,-1.5529794598,-2.2431731016$ $\mathrm{H}, 0,2.4170191,-3.3166111245,-2.3671827364$ $\mathrm{H}, 0,2.3331344664,-2.3266134101,-3.8351718603$ C, $0,0.1087440588,-3.8454778556,-3.7345656762$ $\mathrm{H}, 0,0.5265879491,-3.6866232123,-4.7347746069$ $\mathrm{H}, 0,0.5664868507,-4.7567555581,-3.3311793304$ $\mathrm{H}, 0,-0.966637025,-4.0226355032,-3.855822308$ C,0,4.0104977109,7.1153771115,-1.6795445951 C, $0,3.5094849323,7.8631416758,-2.938380923$ $\mathrm{H}, 0,4.0206490419,8.8283406328,-3.0403171707$ $\mathrm{H}, 0,3.6953207273,7.2921929557,-3.8544227647$ $\mathrm{H}, 0,2.4319254577,8.0541205384,-2.8768235083$ C,0,3.7582862952,8.0170272068,-0.4560975356 
$\mathrm{H}, 0,2.6938759363,8.2495502004,-0.3343406281$

$\mathrm{H}, 0,4.1174280391,7.555523622,0.4712829815$

$\mathrm{H}, 0,4.2908409611,8.9666758775,-0.5790410498$

C, $0,5.5355769536,6.8808508102,-1.7978854458$

$\mathrm{H}, 0,5.921841207,6.3638647466,-0.9117132172$

$\mathrm{H}, 0,5.7881594972,6.2748143685,-2.674686866$

$\mathrm{H}, 0,6.0648024729,7.8370145562,-1.8916615039$

C,0,-2.1140518681,-5.4470717127,0.5598858284

C, $0,-3.3051940963,-5.6608816475,-0.4044899934$

$\mathrm{H}, 0,-2.9863536448,-5.6706132136,-1.4523892417$

$\mathrm{H}, 0,-3.8022369393,-6.6170199622,-0.1992431279$

$\mathrm{H}, 0,-4.046935871,-4.8615002564,-0.2847427553$

C, $0,-2.65612397,-5.4856217263,2.0015903245$

$\mathrm{H}, 0,-1.8548537952,-5.3698507935,2.7404969674$

$\mathrm{H}, 0,-3.4000725689,-4.6997639271,2.1785051506$

$\mathrm{H}, 0,-3.1427114265,-6.4488994962,2.1912422715$

C, $0,-1.1150396748,-6.617367364,0.3948566225$

$\mathrm{H}, 0,-0.7069622795,-6.6643787182,-0.6205510308$

$\mathrm{H}, 0,-0.2763167078,-6.5114694591,1.0935292643$

$\mathrm{H}, 0,-1.6051204772,-7.5768275443,0.6007026492$

Si,0,-7.2579979593,2.7979339997,3.2744418849

C, $0,-3.3074502839,2.8022210975,0.2327033967$

N,0,-4.1070441471,2.8590503244,1.0924667285

$\mathrm{P}, 0,-7.0490809236,0.7491260481,0.8375192424$

$\mathrm{O}, 0,-7.5422107982,1.5087876394,2.1210366984$

C, $0,-6.8450226382,1.8799326349,-0.55633568$

$\mathrm{H}, 0,-6.0194172771,2.5622380749,-0.3309165042$

$\mathrm{H}, 0,-6.5973123812,1.3013755536,-1.4516991498$

$\mathrm{H}, 0,-7.7727310825,2.4356372288,-0.7218309827$

C, $0,-5.5087350888,-0.1397257898,1.146458018$

$\mathrm{H}, 0,-5.2600857056,-0.7430006311,0.2670481705$

$\mathrm{H}, 0,-4.7133947301,0.5940614104,1.314636924$

$\mathrm{H}, 0,-5.6302319235,-0.7888394605,2.0189662423$

C,0,-8.3470861677,-0.4499130408,0.4322366064

$\mathrm{H}, 0,-9.2913165351,0.0721625838,0.2516250109$

$\mathrm{H}, 0,-8.0670282182,-1.0123760664,-0.4647422215$

$\mathrm{H}, 0,-8.4810748492,-1.1449829711,1.2662912217$

C, $0,-8.8100851339,2.7287630438,4.3243343729$

$\mathrm{H}, 0,-8.7668544058,3.4859111277,5.1169968854$

$\mathrm{H}, 0,-9.7095033762,2.9245290228,3.7297077012$

H,0,-8.9283781298, 1.7517756582,4.8064177009

C, $0,-7.0851220122,4.3951922943,2.3145690885$

H,0,-7.0371236803,5.2422964175,3.0107280826

$\mathrm{H}, 0,-6.1583071103,4.3890794623,1.7311044781$

$\mathrm{H}, 0,-7.9347122312,4.5726220567,1.6449578905$

C,0,-5.7061587134,2.3558271653,4.2241082894 
$\mathrm{H}, 0,-4.839390037,2.3984840036,3.5557392467$ $\mathrm{H}, 0,-5.5456419761,3.0785724874,5.0342178125$ $\mathrm{H}, 0,-5.7682132856,1.3602705054,4.6787109331$ C, $0,4.4744140419,-3.72113278,0.1285470717$ C, $0,3.278955152,-4.3779804136,0.465915338$ C, $0,5.3329801208,-4.2869624075,-0.8241260448$ C, $0,2.9491381413,-5.583753324,-0.1498928818$ $\mathrm{H}, 0,2.6032045442,-3.9483131421,1.199730102$ C, $0,4.9974200421,-5.497593493,-1.4341243575$ $\mathrm{H}, 0,6.2558939823,-3.7860560282,-1.0967439853$ C,0,3.8084009701,-6.1457511855,-1.0985372296 H,0,2.0194683481,-6.0811143334,0.1087242667 H,0,5.6659134335,-5.9295927301,-2.1730898815 $\mathrm{H}, 0,3.5495783039,-7.0869145572,-1.5752851059$ C, $0,6.4295902323,-1.5112503452,0.2350669489$ C, $0,7.6824876165,-1.6557783726,0.8494215931$ C, $0,6.3350545306,-0.8587733299,-1.006556123$ C, $0,8.8273580088,-1.1577916593,0.2244278868$ $\mathrm{H}, 0,7.7688250308,-2.1427105365,1.8148941932$ C, $0,7.4835982544,-0.3696613531,-1.6269463604$ $\mathrm{H}, 0,5.3653133396,-0.7248260605,-1.4766492431$ C, $0,8.729985522,-0.5180141059,-1.0123263209$ $\mathrm{H}, 0,9.7936419518,-1.2669303472,0.7082197026$ $\mathrm{H}, 0,7.402400743,0.1336320026,-2.5859023988$ $\mathrm{H}, 0,9.6228228722,-0.1303314384,-1.494571111$ C, $0,5.2423419146,-2.6201825228,2.7081722808$ C, $0,6.0267917582,-3.7499207242,2.998056492$ C, $0,4.70846809,-1.8626859724,3.75904749$ C, $0,6.293016809,-4.0960077688,4.3223076769$ H,0,6.4175051967,-4.3666423629,2.1933775134 C,0,4.9789001365,-2.2124857495,5.0831897115 $\mathrm{H}, 0,4.0684519073,-1.0145709291,3.5386709978$ C, $0,5.7735605329,-3.3246075999,5.3655979585$ H, $0,6.8986262805,-4.9710848291,4.5395605404$ H,0,4.5631126826,-1.619699291,5.8929154669 H,0,5.9802541604,-3.5975101216,6.3965189612

only imaginary frequency: $-216.0 \mathrm{~cm}^{-1}$

$\mathrm{HF}=-4471.5370193$ (A.U.)

Zero-point correction $=1.610447$ (A.U.)

Total Gibbs free energy $=-4470.071913$ (A.U.)

\section{IM2}

Cartesian Coordinates (Angstroms)

C, $0,1.8030639429,-3.7448584362,-0.8712961777$ 
C, $0,0.6701760413,-3.2557284447,-0.2292142088$ C, $0,0.1042052031,-2.0173463861,-0.7071225551$ C, $0,0.7416923614,-1.3807804666,-1.8237690846$ C, $0,1.8842458109,-1.9538223455,-2.4327756212$ C, $0,2.4470388941,-3.130242555,-1.9755173402$ H, $0,2.2248515742,-4.674343729,-0.5075229198$ $\mathrm{H}, 0,2.3070512326,-1.4344128002,-3.2889598148$ C, $0,0.2374035665,-0.1666108141,-2.421350312$ $\mathrm{N}, 0,-0.7154783555,0.570777858,-1.9567560264$ $\mathrm{N}, 0,-2.2721523297,2.1472714452,-0.5115826583$ C, $0,-3.3259397499,2.66009058,0.0414757862$ C, $0,-4.1274937753,2.0586323926,1.0742875819$ C, $0,-3.8147116283,0.7955066994,1.6742440321$ C, $0,-5.2626823727,2.797695709,1.4922047306$ $\mathrm{C}, 0,-4.6992359916,0.2997016711,2.6936248665$ $\mathrm{C}, 0,-6.1142588173,2.338902787,2.4761578459$ $\mathrm{H}, 0,-5.4445556849,3.7511777814,1.0045865391$ C, $0,-5.792774322,1.0817346239,3.0452946401$ $\mathrm{H}, 0,-6.4565107789,0.7054783491,3.8141109707$ $\mathrm{O}, 0,-2.7487607226,0.1122344001,1.3359051325$ $\mathrm{O}, 0,-0.9764958128,-1.5256798328,-0.1624958645$ $\mathrm{Al}, 0,-1.629637122,0.2386056681,-0.140607606$ $\mathrm{H}, 0,0.7273214425,0.119926989,-3.3568388061$ $\mathrm{H}, 0,-3.6674400697,3.6469236962,-0.281118719$ $\mathrm{P}, 0,-4.1250838309,-3.6342937917,-3.1607762039$ C, $0,-5.3195157256,-0.6928499012,-2.2665617779$ $\mathrm{H}, 0,-5.2938040515,0.292838904,-2.7436296372$ $\mathrm{H}, 0,-5.8864752788,-0.5805184671,-1.3357500219$ $\mathrm{H}, 0,-5.855572935,-1.3874900196,-2.9169642084$ $\mathrm{O}, 0,-3.2611222244,-0.2312907922,-1.2493057727$ C, $0,-3.8981652903,-1.1080955345,-1.9194442779$ C, $0,-3.349744374,-2.3379779858,-2.2960467028$ $\mathrm{H}, 0,-2.3443360385,-2.5598215561,-1.9583141817$ C, $0,7.5484376592,-3.3816365671,4.2604578955$ C,0,6.8571481834,-2.3811609295,4.9523956197 C,0,5.7058209778,-1.8166833444,4.4121207343 C, $0,5.212113374,-2.2380840087,3.1601994873$ C,0,5.9176451524,-3.2510091086,2.4816055927 C, $0,7.071948742,-3.8153424424,3.0223430746$ $\mathrm{H}, 0,8.444231433,-3.8225289361,4.6882904447$ $\mathrm{H}, 0,7.2147003305,-2.0465222764,5.9224291499$ $\mathrm{H}, 0,5.1755808793,-1.0547541565,4.9763176772$ C, $0,4.0122652962,-1.6741137687,2.5348632046$ $\mathrm{H}, 0,5.5468104751,-3.5995290882,1.5203185484$ $\mathrm{H}, 0,7.5955658843,-4.5976418024,2.4796248945$ C,0,3.2777921191,-0.6172791156,2.9595583099 
C, $0,2.1347769529,-0.0897668118,2.2379174462$

C, $0,1.5094374106,1.0562056808,2.5548135096$

C, $0,0.3694646707,1.6531911843,1.7174079074$

$\mathrm{O}, 0,-0.0509729542,0.8093701341,0.735720297$

C, $0,-0.7157060977,2.2009734084,2.6741673902$

$\mathrm{H}, 0,3.689086095,-2.1739248262,1.6202000644$

$\mathrm{H}, 0,3.557054572,-0.0970801071,3.8767476917$

$\mathrm{H}, 0,1.7757040732,-0.6351794517,1.3675633004$

$\mathrm{H}, 0,1.8232773102,1.6419016113,3.4199013502$

$\mathrm{H}, 0,-1.484657883,2.7461586825,2.1246766239$

$\mathrm{H}, 0,-1.1832451825,1.3557500234,3.183149316$

$\mathrm{H}, 0,-0.2782910369,2.872461472,3.4221863315$

C, $0,-1.5223338042,2.8293972946,-1.5747151223$

C, $0,-1.2187045114,1.7598206472,-2.659806792$

C, $0,-2.1678606833,4.0746994557,-2.1953365421$

$\mathrm{C}, 0,-0.3399046679,2.3352656844,-3.7775946831$

$\mathrm{H}, 0,-2.1878969869,1.4665825979,-3.0895093737$

C,0,-1.2872859913,4.6472970857,-3.3172673913

$\mathrm{H}, 0,-3.1580505987,3.8152201868,-2.5969642908$

C,0,-0.9816690455,3.5921138912,-4.3879174236

$\mathrm{H}, 0,-0.1835433977,1.5883680139,-4.5656303959$

$\mathrm{H}, 0,0.6509807183,2.5855876236,-3.3711133897$

$\mathrm{H}, 0,-1.7821158046,5.514828955,-3.7695096374$

$\mathrm{H}, 0,-0.3448583625,5.0139496446,-2.8860707165$

$\mathrm{H}, 0,-0.3204649274,4.0082422964,-5.1574313032$

$\mathrm{H}, 0,-1.9141103501,3.3106183618,-4.8977098253$

$\mathrm{H}, 0,-0.5563486222,3.1229761382,-1.139997774$

$\mathrm{H}, 0,-2.3243852681,4.8427202541,-1.4287383505$

$\mathrm{C}, 0,-4.4469724464,-1.0679706223,3.3639869065$

C, $0,-3.082022181,-1.0647809353,4.0946673851$

$\mathrm{H}, 0,-3.0649811614,-0.2954288334,4.876626529$

$\mathrm{H}, 0,-2.9108045945,-2.0352775903,4.5774881848$

$\mathrm{H}, 0,-2.2624920308,-0.8773374484,3.3998521714$

C, $0,-5.5258056942,-1.4161937458,4.410382718$

$\mathrm{H}, 0,-5.2982839927,-2.3944472837,4.848864109$

$\mathrm{H}, 0,-5.5568597028,-0.6904149126,5.2313974821$

$\mathrm{H}, 0,-6.5276169946,-1.4795492461,3.9690566228$

C, $0,-4.464901573,-2.1859747045,2.2923708277$

$\mathrm{H}, 0,-5.4445321143,-2.2247362383,1.7977783506$

$\mathrm{H}, 0,-3.6971347381,-2.0185314079,1.5356119138$

$\mathrm{H}, 0,-4.2853415767,-3.1609233581,2.7637161052$

C, $0,0.0316915543,-4.0181876161,0.9538170559$

C, $0,0.0208959968,-3.1334247844,2.2234143555$

$\mathrm{H}, 0,-0.5080228973,-2.1952602341,2.0527011247$

$\mathrm{H}, 0,-0.4761290539,-3.6677033294,3.0426814149$

$\mathrm{H}, 0,1.0425996014,-2.9055603564,2.5491917708$ 
C,0,-1.4171211234,-4.4303094096,0.5961993864 $\mathrm{H}, 0,-2.0344049725,-3.5566565433,0.3829263196$ $\mathrm{H}, 0,-1.4278608643,-5.0927582061,-0.2772063054$ $\mathrm{H}, 0,-1.8658073368,-4.9747386776,1.4366593747$ C, $0,0.8002382652,-5.3105525912,1.299477847$ $\mathrm{H}, 0,0.3079663785,-5.8037162599,2.1448040593$ $\mathrm{H}, 0,0.8102841716,-6.0239929093,0.466513532$ $\mathrm{H}, 0,1.8364278102,-5.1118419352,1.5984808681$ C, $0,-7.355993056,3.1063757082,2.9621390946$ C, $0,-7.2255004617,3.4037346552,4.4753134633$ $\mathrm{H}, 0,-8.1088782212,3.9442418839,4.8367928448$ $\mathrm{H}, 0,-7.1343214202,2.4859021213,5.0660656374$ H,0,-6.3424799407,4.0205550766,4.6781852807 C, $0,-7.5335674425,4.4475368576,2.2252377685$ $\mathrm{H}, 0,-6.6780465515,5.1150402395,2.3813552275$ $\mathrm{H}, 0,-7.6659800482,4.3052955037,1.1462102883$ $\mathrm{H}, 0,-8.4251609857,4.9615458776,2.6013272576$ C, $0,-8.6242786683,2.2540396196,2.7176199584$ $\mathrm{H}, 0,-8.7541595539,2.043630362,1.6496340982$ $\mathrm{H}, 0,-8.5800940592,1.2946636247,3.2443519444$ $\mathrm{H}, 0,-9.5170315782,2.7847875616,3.0706942983$ C,0,3.6865965233,-3.7865281543,-2.6092103091 C, $0,4.7972028432,-3.95689135,-1.5450784542$ $\mathrm{H}, 0,4.4669410072,-4.5808136019,-0.7077259924$ $\mathrm{H}, 0,5.6825614975,-4.4328655618,-1.9839784855$ $\mathrm{H}, 0,5.1017649105,-2.9841891024,-1.1390401179$ C, $0,4.2624089827,-2.9471307301,-3.7658268793$ $\mathrm{H}, 0,3.5402790749,-2.8247884534,-4.5810276079$ $\mathrm{H}, 0,4.5688701781,-1.948495584,-3.430277685$ $\mathrm{H}, 0,5.1472269381,-3.4416663336,-4.1819107035$ C, $0,3.3132206666,-5.1795741546,-3.1711778053$ $\mathrm{H}, 0,2.9140076511,-5.8374831049,-2.3919427441$ $\mathrm{H}, 0,2.5555778163,-5.0890322569,-3.9587915508$ $\mathrm{H}, 0,4.1925744527,-5.6718530561,-3.6045281984$ C, $0,1.0613725198,2.8385031376,1.0446331489$ $\mathrm{N}, 0,1.6710330979,3.6424233442,0.4596748328$ Si, $0,4.9840767016,4.7668283215,-1.6269259279$ P, $0,5.2030839479,2.030830085,-0.0110364187$ O,0,5.5385124571,3.2506054897,-0.9454575301 C,0,3.8606934159,1.0376223998,-0.6983598391 $\mathrm{H}, 0,4.1068463521,0.7550746507,-1.7262301407$ $\mathrm{H}, 0,3.7305797739,0.1340474814,-0.0959803585$ H, $0,2.9263749111,1.6045853564,-0.6768733133$ $\mathrm{C}, 0,6.699778894,1.0146285838,0.0697053682$ $\mathrm{H}, 0,6.5226406268,0.1478169468,0.7153571478$ H,0,6.9685777125,0.6701708966,-0.9332009053 
$\mathrm{H}, 0,7.5267466806,1.6044028568,0.4758693815$ C,0,4.7890380003,2.5925059221,1.6546484348 $\mathrm{H}, 0,3.8660609534,3.1776311394,1.6176402881$ $\mathrm{H}, 0,4.6335534725,1.7204184324,2.2971141657$ H,0,5.6072959294,3.2031900648,2.0478965786 C,0,4.5390509673,5.9013422892,-0.2054989416 $\mathrm{H}, 0,4.3161738304,6.9038717732,-0.5926348198$ $\mathrm{H}, 0,3.6436577187,5.5462055001,0.3162892463$ $\mathrm{H}, 0,5.35781571,6.0078416743,0.5153882442$ C, $0,3.504226281,4.3717591114,-2.706637648$ H,0,3.1937264248,5.271210152,-3.2533424926 $\mathrm{H}, 0,3.7287046604,3.5977154687,-3.4495186414$ $\mathrm{H}, 0,2.655081989,4.04874483,-2.0940225841$ C, $0,6.4792723508,5.3391248524,-2.5985568297$ $\mathrm{H}, 0,6.7587993226,4.6161333308,-3.3730337894$ $\mathrm{H}, 0,6.2675897888,6.2933435178,-3.0963537124$ H,0,7.3476349178,5.4913994924,-1.9478327768 C, $0,-4.3355662356,-3.3414972688,-4.9604290093$ C,0,-4.5846244411,-4.4108053094,-5.8379737472 C, $0,-4.2152828337,-2.0432129674,-5.473095635$ C,0,-4.7371543725,-4.1751416137,-7.2038554824 $\mathrm{H}, 0,-4.6460054734,-5.4275014056,-5.4596556715$ C, $0,-4.3697219318,-1.8118660904,-6.841166425$ $\mathrm{H}, 0,-3.9831266349,-1.221468345,-4.8030953796$ C, $0,-4.6346709233,-2.8748249988,-7.705938095$ $\mathrm{H}, 0,-4.9280456926,-5.0064742641,-7.8763434908$ $\mathrm{H}, 0,-4.2772004318,-0.8021234951,-7.2309359429$ $\mathrm{H}, 0,-4.7514556013,-2.6938914801,-8.7706697173$ C, $0,-5.7633237855,-4.0824323462,-2.4790452656$ C, $0,-5.900546985,-4.1044323917,-1.080036247$ C, $0,-6.8607851861,-4.4116437994,-3.2884663052$ C,0,-7.1175722643,-4.4647509469,-0.5037153526 H,0,-5.0619903884,-3.826639296,-0.4483740076 C, $0,-8.0780369908,-4.7663501288,-2.7037278636$ $\mathrm{H}, 0,-6.7753651515,-4.3817516714,-4.3694522521$ C, $0,-8.206920453,-4.7959232687,-1.3142105646$ $\mathrm{H}, 0,-7.2155762767,-4.4788363097,0.5778226944$ $\mathrm{H}, 0,-8.9255197902,-5.0141416475,-3.3362604275$ $\mathrm{H}, 0,-9.1560897343,-5.0704070934,-0.8627664245$ C, $0,-3.0489862945,-5.1020617294,-3.0377084004$ C, $0,-1.6905977288,-4.9787847795,-3.3745971243$ C, $0,-3.5550765787,-6.3477096353,-2.6413376289$ C, $0,-0.8524664726,-6.090163222,-3.307392318$ $\mathrm{H}, 0,-1.2841827903,-4.0184124706,-3.6778186667$ C, $0,-2.7102166476,-7.4581196314,-2.5809986704$ H,0,-4.6009365735,-6.4532945888,-2.372870606 
$\mathrm{C}, 0,-1.3611315395,-7.3308248555,-2.9127209062$

$\mathrm{H}, 0,0.1986402508,-5.9850582748,-3.558340628$

$\mathrm{H}, 0,-3.1087355224,-8.4195752657,-2.2705296205$

$\mathrm{H}, 0,-0.7056306177,-8.1957027199,-2.8633014013$

$\mathrm{HF}=-4471.5510069$ (A.U.)

Zero-point correction $=1.612355$ (A.U.)

Total Gibbs free energy $=-4470.081660$ (A.U.)

\section{Reference}

(1) Sigman, M. S.; Jacobsen, E. N. J. Am. Chem. Soc. 1998, 120, 5315.

(2) (a) John, J. A.; Tour, J. M. Tetrahedron 1997, 53, 15515. (b) Li, P.-F.; Wang, H.-L.; Qu, J. J. Org. Chem. 2014, 79, 3955. (c) Kang, J.; Lim, G. J.; Yoon, S. K.; Kim, M. Y. J. Org. Chem. 1995, 60, 564. (d) Danishefsky, S.; Berman, E. M.; Ciufolini, M.; Etheredge, S. J.; Segmuller, B. E. J . A m. Chem. Soc. 1985, 107, 3891. (e) Su, W. K.; Zhuang, Y. G.; Wu, D. Z.; Zhong, W. H. Org. Prep. Proced. Int. 2007, 39, 195. (f) Volz, F.; Wadman, S. H.; Hoffmann-Röder, A.; Krause, N. Tetrahedron 2009, 65, 1902.

(3) Frisch, M. J.; Trucks, G. W.; Schlegel, H. B.; Scuseria, G. E.; Robb, M. A.; Cheeseman, J. R.; Scalmani, G.; Barone,V.; Mennucci, B.; Petersson, G. A.; Nakatsuji, H.; Caricato, M.; Li, X.; Hratchian, H. P.; Izmaylov, A. F.; Bloino, J.; Zheng, G.; Sonnenberg, J. L.; Hada, M.; Ehara, M.; Toyota, K.; Fukuda, R.; Hasegawa, J.; Ishida, M.; Nakajima, T.; Honda, Y.; Kitao, O.; Nakai, H.; Vreven, T.; Montgomery, J. A.; Peralta,Jr., J. E.; Ogliaro, F.; Bearpark, M.; Heyd, J. J.; Brothers, E.; Kudin, K. N.; Staroverov, V. N.; Kobayashi, R.; Normand, J.; Raghavachari, K.; Rendell, A.; Burant, J. C.; Iyengar, S. S.; Tomasi, J.; Cossi, M.; Rega, N.; Millam, J. M.; Klene, M.; Knox, J. E.; Cross, J. B.; Bakken, V.; Adamo, C.; Jaramillo, J.; Gomperts, R.; Stratmann, R. E.; Yazyev, O.; Austin, A. J.; Cammi, R.; Pomelli, C.; Ochterski, J. W.; Martin, R. L.; Morokuma, K.; Zakrzewski, V. G.; Voth, G. A.; Salvador, P.; Dannenberg, J. J.; Dapprich, S.; Daniels, A. D.; Farkas, O.; Foresman, J. B.; Ortiz, J. V.; Cioslowski, J.; Fox, D. J. 2009, Gaussian 09, Revision A. 1, Gaussian, Inc.; Wallingford, CT.

(4) Becke, A. D. J. Chem. Phys. 1993, 98, 5648.

(5) Lee, C.; Yang, W.; Parr, R. G. Phys. Rev. B, 1988, 37, 785.

(6) Hay, P. J.; Wadt, R. J. Chem. Phys. 1985, 82, 299.

(7) (a) Mennucci, B.; Tomasi, J. J. Chem. Phys. 1997, 106, 5151. (b) Mennucci, B.; Cancès, E.; Tomasi, J. J. Phys. Chem. B, 1997, 101, 10506. (c) Tomasi, J.; Mennucci, B.; Cancès, E. J. Mol. Struc. (Theochem), 1999, 464, 211.

(8) Reed, A. E.; Curtiss, L. A.; Weinhold, F. Chem. Rev. 1988, 88, 899.

(9) Denmark, S. E.; Chung, W.-J. J. Org. Chem. 2006, 71, 4002.

(10) For selected examples: (a) Albanese, J. A.; Staley, D. L.; Rheingold, A. L.; Burmeister, J. L. Inorg. Chem. 1990, 29, 2209. (b) Belluco, U.; Michelin, R. A.; Bertani, R.; Facchin, G.; Pace, G.; Zanotto, L.; Mozzon, M.; Furlan, M.; Zangrando, E. Inorg. Chim. Acta. 1996, 252, 355. (c) Spencer, E. C.; Kalyanasundari, B.; Mariyatra, M. B.; Howard, J. A. K.; Panchanatheswaran, K. Inorg. Chim. Acta. 2006, 359, 35. (d) Sabounchei, S. J.; Nemattalab, H.; Salehzadeh, S.; Khani, S.; Bayat, M.; Khavasi, R. H. Polyhedron 2008, 27, 2015. (e) Ebrahim, M. M.; Panchanatheswaran, K.; 
Neels, A.; Stoeckli-Evans, H. J. Organomet. Chem. 2009, 694, 643. (f) Karami, K.; Salah, M. M. Transition Met. Chem. 2011, 36, 363.

(11) (a) Hamashima, Y.; Sawada, D.; Kanai, M.; Shibasaki, M. J. Am. Chem. Soc. 1999, 121, 2641. (b) Vogl, E. M.; Gröger, H.; Shibasaki, M. Angew. Chem. Int. Ed. 1999, 38, 1570. (c) Sawada, D.; Kanai, M.; Shibasaki, M. J. Am. Chem. Soc. 2000, 122, 10521. (d) Casas, J.; Nájera, C.; Sansano, J. M.; Saá, J. M. Org. Lett. 2002, 4, 2589;

(12) Ryu, D. H.; Corey, E. J. J. Am. Chem. Soc. 2004, 126, 8106.

(13) (a) Falvello, L. R.; Fernández, S.; Navarro, R.; Pascual, I.; Urriolabeitia, E. P. J. Chem. Soc. Dalton Trans. 1997, 763. (b) Falvello, L. R.; Fernández, S.; Navarro, R.; Urriolabeitia, E. P. Inorg. Chem. 1996, 35, 3064.

(14) Schweizer, E. E.; Calcagno, M. A. J. Org. Chem. 1977, 42, 2641.

(15) Sabounchei, S. J.; Nemattalab, H.; Salehzadeh, S.; Khani, S.; Bayat, M.; Adams, H.; Ward, M. D. Inorg. Chim. Acta. 2009, 362, 105.

(16) (a) V. Gutmann, The Donor-Acceptor Approach to Molecular Interactions (Plenum Press: New York, 1978). (b) Denmark, S. E.; Beutner, G. L. Angew. Chem., Int. Ed. 2008, 47, 1560.

(17) Tao, J.-C.; Guo, Y.; Li, S.-H. J. Mol. Struc. (Theochem). 2009, 899, 61.

(18) Ping, X.-F.; Zhu, Y.-Q.; Guo, Y.; Xue, Y.; Wang, L.-J.; Feng, X.-M.; Xie, D.-Q. J. Mol. Struc. (Theochem), 2008, 863,60 .

(19) Ryu, D. H.; Corey, E. J. J. Am. Chem. Soc. 2005, 127, 5384.

(20) Shibasaki, M.; Kanai, M.; Funabashi, K. Chem. Commun. 2002, 1989.

(21) Yabu, K.; Masumoto, S.; Yamasaki,S.; Hamashima, Y.; Kanai, M.; Du, W.; Curran, D. P.; Shibasaki, M. J. Am. Chem. Soc. 2001, 123, 9908.

(22) Nanda, S.; Kato, Y.; Asano, Y. Tetrahedron 2005, 61, 10908.

(23) Kim, S. S.; Lee, S. H.; Kwak, J. M. Tetrahedron: Asymmetry 2006, 17, 1165.

(24) Deng, H.; Isler, M. P.; Snapper, M. L.; Hoveyda, A. H. Angew. Chem., Int. Ed. 2002, 41, 1009.

(25) Caille, S.; Cui, S.; Hwang, T.-L.; Wang, X.; Faul, M. M. J. Org. Chem. 2009, 74, 3833.

(26) Maki, K.; Motoki, R.; Fujii, K.; Kanai, M.; Kobayashi, T.; Tamura, S.; Shibasaki, M. J. Am. Chem. Soc. 2005, 127, 17111.

(27) Douglas, F.; Jacobsen, E. N. J. Am. Chem. Soc, 2005, 122, 8964.

(28) Fujii, K.; Maki, K.; Kanai, M.; Shibasaki, M. Org. Lett. 2003, 5, 733. 